\title{
Nonequilibrium dynamical mean-field theory and its applications
}

\author{
Hideo Aoki and Naoto Tsuji \\ Department of Physics, University of Tokyo, Hongo, Tokyo 113-0033, Japan
}

\author{
Martin Eckstein \\ Max Planck Research Department for Structural Dynamics, University of Hamburg-CFEL, \\ Hamburg, Germany \\ Marcus Kollar \\ Theoretical Physics III, Center for Electronic Correlations and Magnetism, Institute of Physics, \\ University of Augsburg, 86135 Augsburg, Germany
}

\author{
Takashi Oka \\ Department of Applied Physics, University of Tokyo, Hongo, Tokyo 113-8656, Japan
}

\author{
Philipp Werner \\ Department of Physics, University of Fribourg, 1700 Fribourg, Switzerland
}

(published 24 June 2014)

\begin{abstract}
The study of nonequilibrium phenomena in correlated lattice systems has developed into one of the most active and exciting branches of condensed matter physics. This research field provides rich new insights that could not be obtained from the study of equilibrium situations, and the theoretical understanding of the physics often requires the development of new concepts and methods. On the experimental side, ultrafast pump-probe spectroscopies enable studies of excitation and relaxation phenomena in correlated electron systems, while ultracold atoms in optical lattices provide a new way to control and measure the time evolution of interacting lattice systems with a vastly different characteristic time scale compared to electron systems. A theoretical description of these phenomena is challenging because, first, the quantum-mechanical time evolution of many-body systems out of equilibrium must be computed and second, strong-correlation effects which can be of a nonperturbative nature must be addressed. This review discusses the nonequilibrium extension of the dynamical mean field theory (DMFT), which treats quantum fluctuations in the time domain and works directly in the thermodynamic limit. The method reduces the complexity of the calculation via a mapping to a selfconsistent impurity problem, which becomes exact in infinite dimensions. Particular emphasis is placed on a detailed derivation of the formalism, and on a discussion of numerical techniques, which enable solutions of the effective nonequilibrium DMFT impurity problem. Insights gained into the properties of the infinite-dimensional Hubbard model under strong nonequilibrium conditions are summarized. These examples illustrate the current ability of the theoretical framework to reproduce and understand fundamental nonequilibrium phenomena, such as the dielectric breakdown of Mott insulators, photodoping, and collapse-and-revival oscillations in quenched systems. Furthermore, remarkable novel phenomena have been predicted by the nonequilibrium DMFT simulations of correlated lattice systems, including dynamical phase transitions and field-induced repulsion-to-attraction conversions.
\end{abstract}

DOI: $10.1103 /$ RevModPhys.86.779

PACS numbers: $71.10 .-\mathrm{W}$

\section{CONTENTS}

I. Introduction

A. Strongly correlated systems out of equilibrium

780

B. Physical background

780

781

II. Methods

A. Nonequilibrium Green's function approach

784

1. Kadanoff-Baym formalism for time evolution from a thermal initial state

784

a. Contour-ordered formulation

b. Contour-ordered Green's functions
784

786 c. Noninteracting contour-ordered Green's functions $\quad 788$ d. Dyson equation $\quad 788$ e. Numerical solution $\quad 789$

2. Keldysh formalism for nonequilibrium steady states

a. Keldysh formalism $\quad 790$

b. Free-fermion bath 791

B. Nonequilibrium dynamical mean-field theory 792

1. Overview of equilibrium DMFT 792

2. Nonequilibrium DMFT formalism 793 
3. Models
a. Overview
b. Time-dependent electric fields
c. Dissipative systems

4. Implementation of the self-consistency

a. Stable time propagation scheme

b. Momentum summations

5. Observables and conservation laws

a. Equal-time observables

b. Photoemission spectrum

c. Optical conductivity

C. Real-time impurity solvers

1. General remarks

2. Falicov-Kimball model

3. Continuous-time quantum Monte Carlo algorithms

a. General remarks

b. Weak-coupling CTQMC

c. Strong-coupling CTQMC

4. Weak-coupling perturbation theory

5. Strong-coupling perturbation theory

a. General remarks

b. Self-consistent strong-coupling equations

c. Numerical implementation

d. Monte Carlo sampling around NCA

D. Floquet formalism for periodically driven systems

1. Overview of Floquet's theorem

2. Floquet Green's function method

a. General formulation

b. Simple example

3. Floquet dynamical mean-field theory

a. General formalism

b. Impurity solver

E. Extensions of DMFT and alternative approaches

1. Cluster perturbation theory

2. Cluster extension of nonequilibrium DMFT

3. Dual fermions

4. Inhomogeneous DMFT

III. Applications

A. Electric fields

1. Overview of field-induced phenomena

2. dc electric fields

a. Bloch oscillations

b. Steady-state current in a dissipative system

c. Dielectric breakdown

3. Photoexcitations and photodoping

4. ac electric fields

a. General remarks

b. Isolated systems

c. Open systems

B. Time-dependent parameter changes

1. Quenches, relaxation, and thermalization

a. Comparison with the thermal state

b. Interaction quench in the Falicov-Kimball model and the role of conserved quantities

c. Interaction quench in the Hubbard model, prethermalization and thermalization

d. Interaction quench in the presence of long-range order

2. Ramps and nonadiabaticity

a. Excitation energy after a continuous parameter change

b. Linear ramps in the Falicov-Kimball model
801

803

803

805

805

805

807

808

808

808

809

809

810

811

811

812

812

812

813

814

815

816

816

816

816

816

817

818

819

820

820

820

822

823

823

823

823 c. Oscillating ramps in the Hubbard model

828

IV. Concluding Remarks and Prospects

828

Acknowledgments

829

Appendix A: Numerical Solution of Volterra

Integral-differential Equation

830

Appendix B: Sample Programs

831

References

832

\section{INTRODUCTION}

\section{A. Strongly correlated systems out of equilibrium}

There is a growing realization that nonequilibrium physics is a major avenue in condensed-matter physics. Of particular interest are nonequilibrium phenomena in strongly correlated electron systems. This class of materials has been intensively studied since the discovery of high- $T_{c}$ superconductivity in the cuprates. Already in equilibrium, strong electronic correlations bring about a tantalizing variety of novel phenomena, such as metal-to-Mott-insulator transitions and transitions to magnetic and superconducting states. If such a system is driven out of equilibrium, we can expect even richer physics, of which only a small fraction has been discovered so far, and of which even less can be considered as being "understood." The present article reviews a recently developed theoretical approach to study those strongly correlated many-body systems out of equilibrium, namely, the nonequilibrium dynamical mean-field theory (DMFT), and illustrates its strength and versatility with numerous applications that have led to new physical insights in several cases.

Over the last two decades, DMFT has greatly contributed to our present understanding of strongly correlated systems in equilibrium, in particular, to Mott physics (Georges et al., 1996; Kotliar et al., 2006). It provides the exact solution of lattice models in the infinite-dimensional limit (Metzner and Vollhardt, 1989). The method treats spatial correlations in a mean-field manner, which allows a self-consistent formulation in terms of an effective single-site impurity problem (Georges and Kotliar, 1992), but accurately treats the temporal quantum fluctuations that are essential for describing strong-correlation phenomena such as the Mott transition. Another virtue of DMFT was realized when Schmidt and Monien (2002) proposed a nonequilibrium generalization of DMFT, by introducing the Keldysh formalism (see Sec. II.A.2) to describe nonequilibrium steady states of correlated electrons driven by time-periodic external fields. While the setup considered in this pioneering paper (a spatially uniform scalar potential) did not correctly capture the effect of an applied electric field, nor the dissipation mechanism which is essential for the description of nonequilibrium steady states (see Sec. II.A.2), it laid the groundwork for the formalism we now call nonequilibrium DMFT. A general formulation of the nonequilibrium DMFT and its application to an electric-field-driven lattice system was then given by Freericks, Turkowski, and Zlatić (2006), who employed the Kadanoff-Baym formalism (see Sec. II.A.1) to describe general transient real-time evolutions from a thermal initial state.

The essential approximation underlying DMFT, both in and out of equilibrium, is the local nature (or momentum 


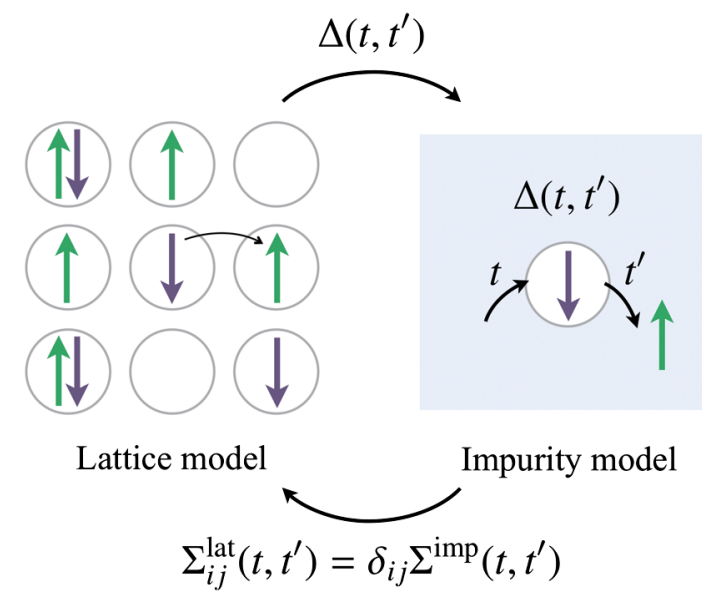

FIG. 1 (color online). A schematic picture of the nonequilibrium DMFT formalism. $\Delta\left(t, t^{\prime}\right)$ is the hybridization function, while $\Sigma\left(t, t^{\prime}\right)$ is the self-energy.

independence) of the self-energy $\Sigma$. This approximation allows one to map the lattice problem onto an impurity problem with a single correlated site embedded in an uncorrelated bath. The hybridization between the impurity site and the bath (the dynamical mean field) is represented by a two-time function $\Delta\left(t, t^{\prime}\right)$, which is subject to a selfconsistency condition (see Fig. 1). Once a self-consistent solution has been obtained, the impurity self-energy $\Sigma^{\text {imp }}$, which is local but time dependent, yields the approximate lattice self-energy, so that the DMFT approximation reads

$$
\sum_{i j}^{\mathrm{lat}}\left(t, t^{\prime}\right) \approx \delta_{i j} \sum^{\mathrm{imp}}\left(t, t^{\prime}\right)
$$

In a time-dependent problem, the self-energy $\Sigma$ becomes a function of two time arguments $\left(t, t^{\prime}\right)$, not just the time difference, and the theory thus incorporates an overall temporal evolution of correlated systems, as well as quantum fluctuations.

The DMFT formalism represents the lattice system as a collection of local entities (atoms or sites) rather than in terms of extended Bloch states, and is thus well suited to treat strong local interactions such as the on-site Hubbard interaction $U$ in a nonperturbative manner. While the reduction from a correlated lattice system to an impurity model is a drastic simplification, the quantum impurity model is still a highly nontrivial many-body system, which must be solved with suitable numerical methods. Over the past several years, the DMFT formalism and various numerical techniques for solving the effective impurity model have been extended to time evolutions (see Sec. II) and subsequently applied to a broad range of problems, including electric-field- and quenchinduced phenomena (see Sec. III).

What are the advantages of the nonequilibrium DMFT over other methods for studying nonequilibrium phenomena in correlated systems? A naive approach would be to solve the time-dependent Schrödinger equation numerically for a many-body wave function, which is, however, quite restricted in terms of the system size, due to exponential growth of the Hilbert space dimension. For one-dimensional systems, the time-dependent density matrix renormalization group
(DMRG) method (Daley et al., 2004; White and Feiguin, 2004; Schollwöck, 2005) and its variants have been widely adopted to accurately simulate the temporal evolution of relatively large (or infinite-size) systems. The restrictions here are the one dimensionality and the accessible time range, which is severely limited, since entanglement grows rapidly in highly excited systems. The nonequilibrium DMFT, by contrast, is formulated directly in the thermodynamic limit and can, in principle and in practice, access longer times. The main limitation of the nonequilibrium DMFT lies in the local approximation for the self-energy, which may not be appropriate in low-dimensional systems where spatially nonlocal correlations can become relevant. These nonlocal correlations may, however, be incorporated into the DMFT formalism through cluster extensions or diagrammatic extensions (Sec. II.E). Within DMFT the nonequilibrium problem is thus approached by starting from a solution which captures the local dynamics in high dimensions correctly, and then trying to build in nonlocal correlations.

An alternative approach that has conventionally been used employs quantum kinetic or quantum Boltzmann equations (Rammer, 1998), based on the nonequilibrium Green's function formalism. It is usually derived from a weakcoupling perturbation expansion, in combination with a semiclassical approximation or gradient expansion. On long time scales, quantum Boltzmann equations describe the relaxation toward a thermal state, while the fast dynamics on short time scales is not captured. While nonequilibrium DMFT can be compared or combined with these methods, it has the advantage that it is nonperturbative and can capture both the short-time and long-time evolutions for any strength of the interaction.

\section{B. Physical background}

Before we start the detailed discussion of nonequilibrim DMFT, we briefly overview the evolution of nonequibrium physics in a broader context. The previous decade has witnessed a remarkable development in the field of ultrafast time-resolved spectroscopies in solids, in which an intense pump laser pulse is used to drive the system into highly excited states, while the temporal evolution of the system is tracked with subsequent probe pulses. The "pump-probe" technique has enabled the study of excitation and relaxation processes in correlated electron systems on their intrinsic microscopic time scale, defined by the electron hopping between the crystal lattice sites (Wall et al., 2011). In strongly correlated materials, quantum fluctuations, inherent in correlated electronic states, are highly intertwined, which makes it difficult to resolve the origin of given physical properties. Real-time spectroscopy introduces a "new dimension" on top of energy and momentum, and can provide an additional perspective on the correlated system by disentangling complicated electronic and lattice processes in, e.g., the cuprates (Dal Conte et al., 2012). Often, the relaxation pathways in complex materials are not at all intuitive, and their study may lead to new concepts for the description and understanding of quantum many-body systems with no simple relation to the familiar equilibrium physics. Pioneering experiments in the field include photoinduced insulator-to-metal transitions in 

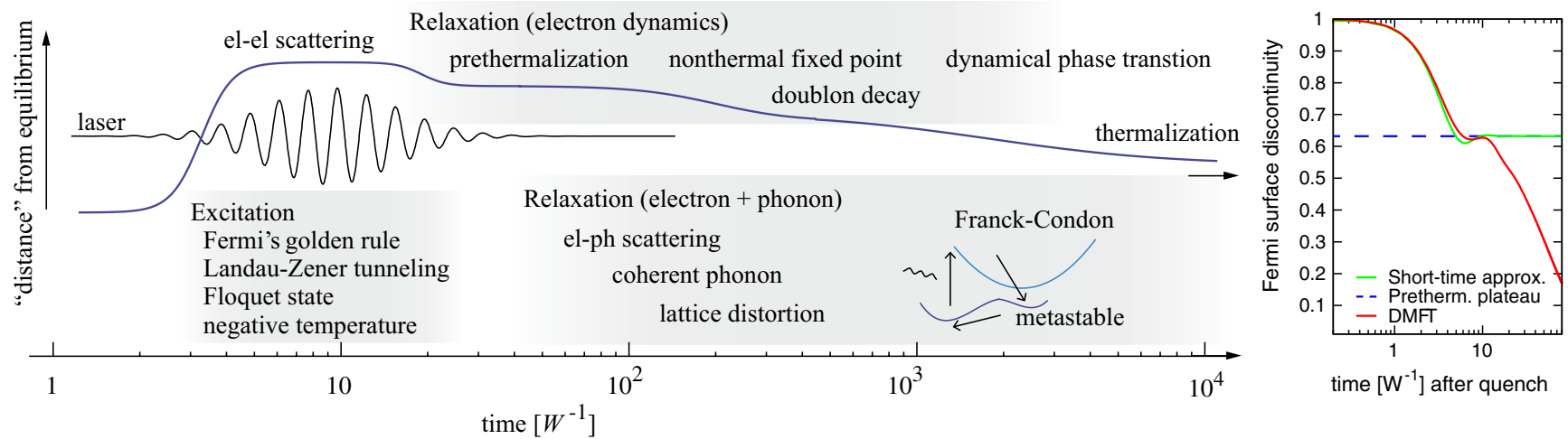

FIG. 2 (color online). Left panel: Schematic time evolution of the system in a pump-probe experiment with various physical processes (see text). Right panel: Comparison of a short-time approximation (Moeckel and Kehrein, 2008; Eckstein, Kollar, and Werner, 2010), which approaches a prethermalization plateau, and nonequilibrium DMFT (Tsuji and Werner, 2013), which also describes the crossover toward a thermal state, for a sudden switching on of the Hubbard interaction to $U=0.375 W$.

correlated Mott and charge-transfer insulators (Ogasawara et al., 2000; Iwai et al., 2003; Perfetti et al., 2006; Kübler et al., 2007; Okamoto et al., 2007, 2008), the pump-induced melting and recovery of charge density waves (Schmitt et al., 2008; Hellmann et al., 2010; Petersen et al., 2011) with studies combining structural and electronic dynamics (Eichberger et al., 2010), and ultrafast dynamics induced in ferromagnets (Beaurepaire et al., 1996) or antiferromagnets (Ehrke et al., 2011), to name only a few.

Remarkably, ultrafast pump-probe spectroscopies have not only unveiled the response to strong external fields, but also provide means to manipulate phases of correlated electron systems. One manifestation of strong correlations, in equilibrium, is the Mott insulator, where the large cost in energy of putting two electrons on the same site leads to a charge excitation gap and inhibits conduction. Using an intense laser pulse, one can excite electrons across the charge gap, which drives the system into a nonequilibrium but relatively longlived conducting state (Ogasawara et al., 2000; Iwai et al., 2003; Perfetti et al., 2006; Okamoto et al., 2007). Such a process, sometimes called photodoping (Nasu, 2004), is a typical example of a pathway to new phases, where mobile carriers are introduced in situ, as distinct from techniques employed in equilibrium, where the carrier concentration is typically controlled by chemical doping (Imada, Fujimori, and Tokura, 1998).

A major difficulty in describing strongly correlated systems is the large dimension of the Hilbert space, which is a problem in equilibrium and becomes an even more serious challenge in nonequilibrium. One factor that makes the treatment of timeevolving quantum many-body systems challenging is the wide range of relevant time scales. One might first expect that strong interactions would help to quickly restore an equilibrium state after a perturbation, due to fast interparticle scattering. However, contrary to the naive expectation, the dynamics of correlated systems generally exhibits a variety of time scales, which can be orders of magnitude different from the intrinsic microscopic time scale of the system, as sketched in the left panel of Fig. 2. The initial dynamics of a system excited by pumping is governed by the electronic degrees of freedom. The excitation during photoirradiation takes place via Fermi's golden rule (linear-response theory) or the Schwinger mechanism (Landau-Zener tunneling in strong fields), depending on whether or not the photon energy is larger than the energy gap. During the laser application, the system may also reach a nonequilibrium time-periodic steady state (a so-called Floquet state; see Sec. II.D) for which the effective (temporal-Fourier transformed) Hamiltonian can drastically differ from the original one.

After the pulse irradiation, electronic relaxation processes set in (Fig. 2). In Mott insulators, e.g., doublons (doubly occupied sites) and holes, which are created in the first stage, start to annihilate in pairs. The relaxation time of doublons in a gapped system scales as (Strohmaier et al., 2010) $\tau \propto W^{-1} \exp [\alpha(U / W) \ln (U / W)]$, where $U$ is the on-site Coulomb repulsion, $W$ is the electronic bandwidth, and $\alpha \sim O(1)$ a dimensionless constant. Thus one can see that there emerges a new time scale, which can be orders of magnitude longer than the intrinsic time scales $\left(W^{-1}\right.$ and $\left.U^{-1}\right)$. Even in the course of thermalization of correlated metals, different time scales may emerge due to prethermalization (Berges, Borsányi, and Wetterich, 2004), the passage by nonthermal fixed points (Berges, Rothkopf, and Schmidt, 2008), and dynamical phase transitions (Sec. III.B). At a certain point, the relaxation process enters a second phase [Relaxation (electron + phonon) in Fig. 2], where classical degrees of freedom such as lattice distortions start to play a role. This regime can be understood within the Frank-Condon picture (Nasu, 2004) (inset of Fig. 2). New time scales can also appear through criticality in the dynamics of long-range order, such as spin-density waves or superconductivity, which may behave classically on a long time scale (time-dependent Ginzburg-Landau picture), but are predicted to traverse through metastable supercritical phases (Mathey and Polkovnikov, 2010; Tsuji, Eckstein, and Werner, 2013) on intermediate time scales.

Another unique feature of correlated systems is that an external perturbation may cause cooperative changes through many-body interactions, and even drive the system into hidden states which are not accessible via adiabatic or thermal pathways (Ichikawa et al., 2011). While photodoping often puts the system in a highly excited state in which the effect of 
correlations can be smeared, more recently much lower photon energies (in the terahertz range) are being used to control material properties by selectively driving certain optical phonon modes. With this technique it is possible to control metal-insulator transitions (Rini et al., 2007; Caviglia et al., 2012), or to induce superconductivity in a stripe-ordered cuprate (Fausti et al., 2011) on ultrafast time scales. An intriguing further step in this direction would be to stabilize otherwise unstable many-body states by a continuous driving, and thus design material properties by external modulations. An example of this type of nonequilibrium control, namely, the sign conversion of the interaction strength by ac electric fields, will be discussed in Sec. III.A.4.

The nonequilibrium phenomena and underlying concepts considered here are quite universal. One manifestation of this universality is the fact that phenomena known from condensedmatter physics are now being realized with cold atomic gases in optical lattices (Bloch, Dalibard, and Zwerger, 2008). Although these dilute gases are a totally different class of systems, they provide an almost ideal realization of the many-body lattice models that have long been studied as low-energy effective theories of real materials. Cold gases are unique in terms of their controllability, which is currently unthinkable in electron systems. For example, one can tune the interparticle interaction almost arbitrarily using a Feshbach resonance, or by changing the lattice potential depth, and thus realize the Mott metalinsulator transition for both bosonic (Greiner et al., 2002) and fermionic (Jördens et al., 2008; Schneider et al., 2008) atomic systems. The basic time scale for the temporal evolution is orders of magnitudes longer $(\sim 1 \mathrm{~ms})$ than that for correlated electron systems ( $\sim 1 \mathrm{fs})$, making it much easier to keep track of the time evolution. Furthermore, cold-atom systems may usually be regarded as isolated from the environment on the time scale of typical experiments. These unique properties make cold-atom systems a valuable testing ground for the study of nonequilibrium physics.

Figure 3 shows an obvious parallel between condensedmatter and cold-atom systems. The top panel plots the number of excited carriers in a Mott insulator as a function of time after photoexcitation (Iwai et al., 2003), while the bottom panel plots the relaxation of the double occupancy, i.e., the probability that a single site is occupied by two fermions with opposite (hyperfine)spins, generated by a periodic modulation of the optical lattice (Strohmaier et al., 2010). Doubly occupied sites play the role of carriers in a Mott insulating background, so that the two panels plot essentially the same quantity. While it has been difficult to measure the doublon density directly in electronic systems, one can see for the coldatom system that the double occupancy decays exponentially, and that the relaxation time changes significantly as the interaction strength is varied. In both systems the bottleneck for the decay of the excited carriers is the transformation of a high-energy excitation into many low-energy excitations via many-body processes (Sensarma et al., 2010; Lenarčič and Prelovšek, 2013). Although the absolute time scales in the two systems are vastly different, a physical understanding of basic nonequilibrium phenomena can thus be developed along similar lines. Furthermore, with cold gases in optical lattices, it has been demonstrated that nonperturbatively strong external fields of oscillating (Struck et al., 2011) or dc nature
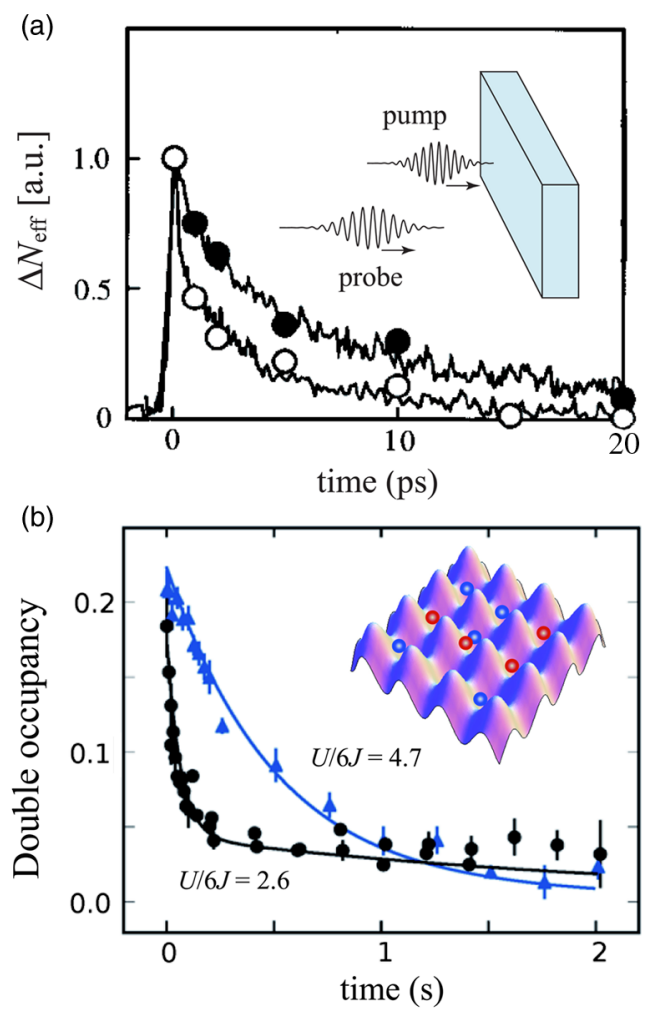

FIG. 3 (color online). (a) Temporal evolution of the density of photoinduced carriers in a correlated electron system (a $\mathrm{Ni}$ complex) for two values of the excitation density. From Iwai et al., 2003. The inset schematically shows a pump-probe experiment. (b) Temporal evolution of the doublon occupation in a cold-atom system on an optical lattice (as schematically depicted in the inset). From Strohmaier et al., 2010.

(Simon et al., 2011) can be used not only to change the state of the system, but to modify its microscopic Hamiltonian in a controlled fashion. While this is yet to be realized for condensed-matter systems, interdisciplinary interactions between the fields of condensed-matter and cold-atom physics may help to achieve this goal in the near future.

The study of nonequilibrium many-body physics extends to broad areas involving high-energy physics, as stressed in the concluding section of this review. A long-standing issue, in both condensed-matter and high-energy physics, concerns the thermalization in isolated quantum systems (Deutsch, 1991; Srednicki, 1994; Rigol, Dunjko, and Olshanii, 2008; Polkovnikov et al., 2011). It is a highly nontrivial and deep question how and when thermalization takes place as a result of the unitary time evolution of a quantum system. Integrable systems usually do not relax to the Gibbs ensemble, but rather to a generalized Gibbs ensemble (Rigol et al., 2007) which also fixes the large number of constants of motion on average. Motivated by cold-atom experiments, these questions have been addressed particularly in the context of quantum "quench" problems (Calabrese and Cardy, 2006; Cazalilla, 2006; Kollath, Läuchli, and Altman, 2007; Manmana et al., 2007; Eckstein and Kollar, 2008b; Moeckel and Kehrein, 2008; Barmettler et al., 2009; Eckstein, Kollar, and Werner, 2009; Dziarmaga, 2010; Cassidy, Clark, and Rigol, 2011; Polkovnikov et al., 2011), where a parameter in the 
Hamiltonian is suddenly changed to generate a nonequilibrium dynamics. After a quench, correlated systems often exhibit "prethermalization" (Berges, Borsányi, and Wetterich, 2004; Moeckel and Kehrein, 2008), i.e., relax to a state in which certain local observables look nearly thermalized, even though the whole momentum distribution still deviates from the thermal one. The right panel of Fig. 2 shows the Fermi surface discontinuity in the momentum distribution after a moderately large interaction quench in the Hubbard model in infinite dimensions. The weak-coupling expansion (Moeckel and Kehrein, 2008; Eckstein, Kollar, and Werner, 2010) describes the transient behavior up to the prethermalization time scale. While the nonequilibrium DMFT result (Tsuji and Werner, 2013) agrees with the perturbative treatment for short times, it also describes the crossover toward the thermal state. This thermalization process may be approximately reproduced by a quantum kinetic approach (Stark and Kollar, 2013). We will discuss these topics in more detail in Sec. III.B.

Another arena of nonequilibrium physics is correlated systems in strong dc fields. There, one of the simplest questions, in the regime beyond the linear response, is to ask what will happen when we apply a strong electric field to an insulator. While a weak field causes only a polarization of the system, stronger fields will induce a dielectric breakdown, and lead to a nonzero current. In a band insulator (Oka and Aoki, 2009a), valence and conduction bands may be modeled, around the band gap, by a two-band Hamiltonian for the valence and conduction bands. When we apply a constant electric field $\boldsymbol{E}$, the wave vector evolves, in the Bloch picture, according to $\boldsymbol{k}=\boldsymbol{k}(0)-e \boldsymbol{E} t / \hbar$ (in a temporal gauge with a vector potential taking care of the field). Nonadiabatic transitions from the lower to the upper band can thus occur, in accord with the nonadiabatic Landau-Zener quantum tunneling (Landau, 1932; Zener, 1932), when the field exceeds a scale set by the gap. The situation is totally different for the breakdown in correlated electron systems, where the relevant gap is a many-body (Mott) gap. Here the theoretical description becomes a formidable problem, since there are two nonperturbative effects involved: the Landau-Zener tunneling which is already nonperturbative (with regards to the electric field $E$ ), and the Mott transition which is also nonperturbative (with regards to the interaction $U$ ). The dielectric breakdown in Mott insulators can then be understood as a field-induced quantum tunneling of many-body states across the Mott gap, which results in a finite doublon-hole creation rate in a strong field (Oka, Arita, and Aoki, 2003; Oka and Aoki, 2005, 2009a, 2010; Oka et al., 2005). Hence there is a continuous crossover from the ac laser excitation ( $\sim$ photon energy $\Omega$ ) across the gap, to the physics in strong dc fields with field strength $E$ : quantum tunneling dominates the nonlinear dc regime, while (generally multi-) photon absorption dominates the ac regime.

One of the ultimate goals in the field of strongly correlated nonequilibrium physics is to induce some kind of long-range "order" that emerges in systems driven out of equilibrium. In this context, an important and still open theoretical issue is how to characterize a nonequilibrium phase transition and the associated critical behavior (Hohenberg and Halperin, 1977). It has been argued that there exist certain universality classes for quantum phase transitions in low dimensional systems driven out of equilibrium (Feldman, 2005; Mitra et al., 2006).
In addition to the criticality at the phase transition point, one may further pose the question of whether one can realize quasistationary "nonequilibrium phases" that are thermally inaccessible through adiabatic pathways. One idea along this line is the concept of a "nonthermal fixed point" (Berges, Rothkopf, and Schmidt, 2008), where the system does not immediately relax to a thermal final state after excitation, but is trapped for a while in a nonthermal quasisteady state.

\section{METHODS}

\section{A. Nonequilibrium Green's function approach}

There exists a variety of methods to deal with the problem of a time-evolving quantum many-body system, ranging from direct wave-function-based techniques, such as exact diagonalization and DMRG, quantum master equations (Breuer and Petruccione, 2002), or quantum kinetic equations (Rammer, 1998), to the Keldysh formalism for nonequilibrium Green's functions (Schwinger, 1961; Kadanoff and Baym, 1962; Keldysh, 1964). The nonequilibrium Green's function method is an extension of the standard equilibrium formulation on the imaginary-time axis (Abrikosov, Gorkov, and Dzyaloshinski, 1975). Using the Keldysh formalism, many theoretical techniques which have been developed for the study of strongly correlated systems, including DMFT, can be straightforwardly adapted to nonequilibrium on a formal level.

The nonequilibrium Green's function approach is applicable to arbitrary time evolutions of correlated systems, and does not involve any assumption on the statistical distribution of particles out of equilibrium, since the time evolution of the distribution function is determined by the initial condition (initial-value problem). A different formulation is needed if one focuses on nonequilibrium steady states of open systems, where driving by an external force is balanced by dissipation to an external heat bath. By assuming that the system has arrived at a nonequilibrium steady state, so that the Green's functions do not change any more as a function of "average time," they are determined by the boundary condition introduced by the heat bath (boundary-value problem). In this case, the formulation is greatly simplified because one can drop the average-time dependence of Green's functions as well as correlations between the time-evolving state and the initial state (initial correlations). We will review the general formulation of nonequilibrium Green's functions (Kadanoff-Baym formalism) in Sec. II.A.1 and then discuss a more specific formulation (Keldysh formalism) for nonequilibrium steady states in Sec. II.A.2.

\section{Kadanoff-Baym formalism for time evolution from a thermal initial state}

\section{a. Contour-ordered formulation}

Consider a general quantum system driven out of equilibrium by an external field, whose time evolution is described by a time-dependent Hamiltonian $H(t)$. Initially (at $t=0$ ) the system is assumed to be in a mixed state described by a density matrix

$$
\rho(0)=\frac{1}{Z} e^{-\beta \mathcal{H}(0)}
$$


where $\beta=1 / T$ is the inverse temperature (with $k_{B}=1$ ), $\mathcal{H}(t)=H(t)-\mu N(t)[\mu$ is the chemical potential, $N(t)$ is the number operator for the particles], and $Z=\operatorname{Tr} e^{-\beta \mathcal{H}(0)}$ is the equilibrium partition function. At $t=0$ we switch on a driving field, and the system starts to evolve from its initial state. The time evolution of the density matrix is determined by the von Neumann equation

$$
i \frac{d}{d t} \rho(t)=[\mathcal{H}(t), \rho(t)],
$$

where the bracket [,] represents the commutator, and $\hbar=1$. Formally, one can write down the solution of Eq. (3) as

$$
\rho(t)=U(t, 0) \rho(0) U(0, t)
$$

where we have defined the unitary evolution operator

$$
U\left(t, t^{\prime}\right)= \begin{cases}\mathcal{T} \exp \left(-i \int_{t^{\prime}}^{t} d \bar{t} \mathcal{H}(\bar{t})\right) & t>t^{\prime} \\ \overline{\mathcal{T}} \exp \left(-i \int_{t^{\prime}}^{t} d \bar{t} \mathcal{H}(\bar{t})\right) & t<t^{\prime}\end{cases}
$$

Here $\mathcal{T}(\overline{\mathcal{T}})$ denotes the (anti-)time-ordering operator, i.e., it arranges the operators so that an operator with time argument $t$ comes left (right) to operators with earlier time arguments $t^{\prime}$ $<t$. Note that the Hamiltonians at different times in general do not commute with each other, $\left[\mathcal{H}(t), \mathcal{H}\left(t^{\prime}\right)\right] \neq 0$. With this ordering, the evolution operator satisfies a fusion rule $U\left(t, t^{\prime}\right) U\left(t^{\prime}, t^{\prime \prime}\right)=U\left(t, t^{\prime \prime}\right)$ and becomes unitary $U\left(t, t^{\prime}\right) \times$ $\left[U\left(t, t^{\prime}\right)\right]^{\dagger}=U\left(t, t^{\prime}\right) U\left(t^{\prime}, t\right)=1$.

Using the time-dependent density matrix (4), the expectation value of an observable $\mathcal{O}$ measured at time $t$ is given by

$$
\langle\mathcal{O}(t)\rangle=\operatorname{Tr}[\rho(t) \mathcal{O}] .
$$

By substituting $\rho(0)$ into Eq. (4) with Eq. (2) and considering $\rho(0)$ as the evolution along the imaginary-time axis from 0 to $-i \beta$ (with imaginary-time ordering), we can express Eq. (6) in a more convenient form,

$$
\begin{aligned}
\langle\mathcal{O}(t)\rangle & =\frac{1}{Z} \operatorname{Tr}\left[U(t, 0) e^{-\beta \mathcal{H}(0)} U(0, t) \mathcal{O}\right] \\
& =\frac{1}{Z} \operatorname{Tr}[U(-i \beta, 0) U(0, t) \mathcal{O} U(t, 0)] .
\end{aligned}
$$

In the second line, we permuted the operators under the trace. If one reads the operators from right to left, one can see that the operators follow the time ordering of $0 \rightarrow t \rightarrow 0 \rightarrow-i \beta$. This motivates us to adopt an L-shaped contour $\mathcal{C}$ with three branches $\mathcal{C}_{1}: 0 \rightarrow t_{\max }, \mathcal{C}_{2}: t_{\max } \rightarrow 0$, and $\mathcal{C}_{3}: 0 \rightarrow-i \beta$, as shown in Fig. 4, where $t_{\max }$ is the maximal time up to which one wants to let the system evolve (Kadanoff and Baym, 1962). Then the expectation value (7) can be written as

$$
\langle\mathcal{O}(t)\rangle=\frac{\operatorname{Tr}\left[\mathcal{T}_{C} e^{-i \int_{c} d \bar{t} \mathcal{H}(\bar{t})} \mathcal{O}(t)\right]}{\operatorname{Tr}\left[\mathcal{T}_{C} e^{-i \int_{C} d \bar{t} \mathcal{H}(\bar{t})}\right]},
$$

where $\mathcal{T}_{\mathcal{C}}$ is a contour-ordering operator that arranges operators on the contour $\mathcal{C}$ in the order $0 \rightarrow t_{\max } \rightarrow 0 \rightarrow-i \beta$ (as indicated by the arrows in Fig. 4), $\mathcal{O}(t)$ indicates that the operator $\mathcal{O}$ is inserted at time $t$ on the contour $\mathcal{C}$ (we are

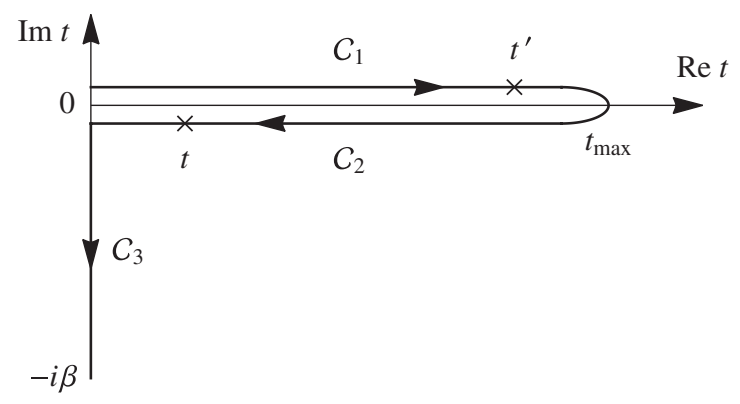

FIG. 4. The L-shaped contour $\mathcal{C}=\mathcal{C}_{1} \cup \mathcal{C}_{2} \cup \mathcal{C}_{3}$ in the KadanoffBaym formalism. The arrows indicate the contour ordering. For example, $t$ lies ahead of $t^{\prime}$ in the ordering $\left(t \succ t^{\prime}\right)$.

working in the Schrödinger picture), and we used the fact that the evolution along the forward $\left(\mathcal{C}_{1}\right)$ and backward $\left(\mathcal{C}_{2}\right)$ contours cancels if no other operator is inserted, so that $e^{-\beta \mathcal{H}(0)}=\mathcal{T}_{C} \exp \left[-i \int_{C} d \bar{t} \mathcal{H}(\bar{t})\right]$.

The contour-ordered formalism reveals its full power when it is applied to higher-order correlation functions

$$
\left\langle\mathcal{T}_{C} \mathcal{A}(t) \mathcal{B}\left(t^{\prime}\right)\right\rangle \equiv \frac{1}{Z} \operatorname{Tr}\left[\mathcal{T}_{C} e^{-i \int_{C} d \bar{t} \mathcal{H}(\bar{t})} \mathcal{A}(t) \mathcal{B}\left(t^{\prime}\right)\right]
$$

Here $\mathcal{A}$ and $\mathcal{B}$ are combinations of particle creation and annihilation operators. We call them "fermionic" if they contain an odd number of the fermion creation or annihilation operators, and "bosonic" otherwise. In this expression, $t$ and $t^{\prime}$ can lie anywhere on $\mathcal{C}$, and the contour-ordered product of two operators $\mathcal{A}$ and $\mathcal{B}$ is defined as

$$
\mathcal{T}_{C} \mathcal{A}(t) \mathcal{B}\left(t^{\prime}\right)=\theta_{\mathcal{C}}\left(t, t^{\prime}\right) \mathcal{A}(t) \mathcal{B}\left(t^{\prime}\right) \pm \theta_{\mathcal{C}}\left(t^{\prime}, t\right) \mathcal{B}\left(t^{\prime}\right) \mathcal{A}(t)
$$

where $\theta_{C}\left(t, t^{\prime}\right)=1$ when $t^{\prime}$ comes earlier than $t$ in the contour ordering (denoted by $t \succ t^{\prime}$; see Fig. 4) and 0 otherwise $\left(t \prec t^{\prime}\right)$. The sign \pm is taken to be minus when the operators $\mathcal{A}$ and $\mathcal{B}$ are both fermionic and plus otherwise. Whenever an operator appears in a contour-ordered product, one has to specify which branch its time argument lies on. For $t=t^{\prime}$ (on the same branch of $\mathcal{C}$ ), we adopt a normal ordering convention, which puts all creation operators to the left of all annihilation operators (Abrikosov, Gorkov, and Dzyaloshinski, 1975), unless the ordering is irrelevant (when $t$ or $t^{\prime}$ is integrated over) or explicitly indicated.

Contour-ordered correlation functions provide a concise way to keep track of both spectral information and occupation functions in a many-particle system out of equilibrium. Before explaining this in further detail (Sec. II.A.1.b), we comment on the relation between the formulation presented here and the one used in field theories at zero temperature, where one usually works with a single-branch time axis ranging from $-\infty$ to $\infty$. The latter is possible due to Gell-Mann and Low's theorem (Gell-Mann and Low, 1951; Fetter and Walecka, 2003), which states that the ground states $|\Psi(0)\rangle$ and $|0\rangle$ of the interacting and noninteracting systems are related by $|\Psi(0)\rangle=U(0,-\infty)|0\rangle$, where the interaction is adiabatically turned on from $t=-\infty$ to $t=0$ (the ground states are 
assumed to be nondegenerate). Then the expectation value (6) is given by

$$
\langle\mathcal{O}(t)\rangle=\left\langle 0\left|U\left(-\infty, t_{\max }\right) U\left(t_{\max }, t\right) \mathcal{O}(t) U(t,-\infty)\right| 0\right\rangle .
$$

We can similarly assume that the interaction is adiabatically switched off in the far future and let $t_{\max } \rightarrow \infty$. Then, the wave function goes back to the noninteracting ground state $|0\rangle$ up to a phase factor $e^{i L}$ ( $L$ is a real number) (Gell-Mann and Low, 1951 and Fetter and Walecka, 2003): $U(\infty,-\infty)|0\rangle=e^{i L}|0\rangle$. Taking its Hermite conjugate and inserting it into Eq. (11) gives an expression for the expectation value

$$
\langle\mathcal{O}(t)\rangle=\frac{\left\langle 0\left|\mathcal{T} e^{-i \int_{-\infty}^{+\infty} d \bar{t} \mathcal{H}(\bar{t})} \mathcal{O}(t)\right| 0\right\rangle}{\left\langle 0\left|\mathcal{T} e^{-i \int_{-\infty}^{+\infty} d \bar{t} \mathcal{H}(\bar{t})}\right| 0\right\rangle}
$$

in which the time argument $t \in(-\infty, \infty)$ moves on a single branch of the real-time axis. However, for general nonequilibrium systems one cannot use Eq. (12), since the initial state $|0\rangle$ would be driven into excited states and never return after the whole time evolution, i.e., $U(\infty,-\infty)|0\rangle \neq e^{i L}|0\rangle$. This forces one to use the analogy of Eq. (8) instead of Eq. (12), with a round trip $(-\infty \rightarrow \infty \rightarrow-\infty)$, and $\langle 0|\cdots| 0\rangle$ instead of the Tr. The idea of this multibranch formalism was originally introduced by Schwinger (1961) and Keldysh (1964). They assumed that many-body interactions are adiabatically switched on from a noninteracting initial state, so that there should be no correlation between the initial state and the time-evolving state. Under this condition it is sufficient to consider two branches $\mathcal{C}_{1}$ and $\mathcal{C}_{2}$ for the time axes (Kamenev, 2011). After that, their approach has been extended to arbitrary initial states with initial correlations taken into account (Danielewicz, 1984a, 1984b; Wagner, 1991) by employing the triple-branch contour depicted in Fig. 4.

\section{b. Contour-ordered Green's functions}

Single-particle Green's functions are the fundamental objects of many-body theories. They describe single-particle excitations as well as statistical distributions of particles, and play a central role in the formulation of nonequilibrium DMFT, which will be reviewed in Sec. II.B. We define the nonequilibrium Green's function as the contour-ordered expectation value

$$
G\left(t, t^{\prime}\right) \equiv-i\left\langle\mathcal{T}_{C} c(t) c^{\dagger}\left(t^{\prime}\right)\right\rangle
$$

where $c^{\dagger}(c)$ is a creation (annihilation) operator of particles, and $t, t^{\prime} \in \mathcal{C}$. For simplicity, spin and orbital indices associated with the operators are not shown. Because of the three branches, on which the time arguments $t$ and $t^{\prime}$ can lie, the Green's function has $3 \times 3=9$ components: $G\left(t, t^{\prime}\right) \equiv$ $G_{i j}\left(t, t^{\prime}\right)\left(t \in \mathcal{C}_{i}, t^{\prime} \in \mathcal{C}_{j}, i, j=1,2,3\right)$. Conventionally we express them in a $3 \times 3$ matrix form

$$
\hat{G}=\left(\begin{array}{lll}
G_{11} & G_{12} & G_{13} \\
G_{21} & G_{22} & G_{23} \\
G_{31} & G_{32} & G_{33}
\end{array}\right) .
$$

In general, one can shift the operator with the largest real-time argument (e.g., $t^{\prime}$ in Fig. 4) from $C_{1}$ to $\mathcal{C}_{2}$ (and vice versa), because the time evolution along $\mathcal{C}_{1}$ and $\mathcal{C}_{2}$ to the right of that operator cancels. This kind of redundancy implies the following relations among the components of the matrix (14):

$$
\begin{gathered}
G_{11}\left(t, t^{\prime}\right)=G_{12}\left(t, t^{\prime}\right) \quad\left(\text { for } t \leq t^{\prime}\right), \\
G_{11}\left(t, t^{\prime}\right)=G_{21}\left(t, t^{\prime}\right) \quad\left(\text { for } t>t^{\prime}\right), \\
G_{22}\left(t, t^{\prime}\right)=G_{21}\left(t, t^{\prime}\right) \quad\left(\text { for } t<t^{\prime}\right), \\
G_{22}\left(t, t^{\prime}\right)=G_{12}\left(t, t^{\prime}\right) \quad\left(\text { for } t \geq t^{\prime}\right), \\
G_{13}\left(t, \tau^{\prime}\right)=G_{23}\left(t, \tau^{\prime}\right), \\
G_{31}\left(\tau, t^{\prime}\right)=G_{32}\left(\tau, t^{\prime}\right) .
\end{gathered}
$$

Equations (15a)-(15d) can be summarized as

$$
G_{11}+G_{22}=G_{12}+G_{21}
$$

The violation of this relation at $t=t^{\prime}$ in the normal ordering convention is negligible under the time integrations used below. Equations (15) thus allow one to eliminate three components out of nine in the Green's function (14). To this end, we introduce six linearly independent physical Green's functions, called the retarded $\left(G^{R}\right)$, advanced $\left(G^{A}\right)$, Keldysh $\left(G^{K}\right)$, left-mixing $\left(G^{\urcorner}\right)$, right-mixing $\left(G^{\ulcorner}\right)$, and Matsubara Green's functions $\left(G^{M}\right)$. They are explicitly given by

$$
\begin{aligned}
G^{R}\left(t, t^{\prime}\right) & =\frac{1}{2}\left(G_{11}-G_{12}+G_{21}-G_{22}\right) \\
& =-i \theta\left(t-t^{\prime}\right)\left\langle\left[c(t), c^{\dagger}\left(t^{\prime}\right)\right]_{\mp}\right\rangle, \\
G^{A}\left(t, t^{\prime}\right) & =\frac{1}{2}\left(G_{11}+G_{12}-G_{21}-G_{22}\right) \\
& =i \theta\left(t^{\prime}-t\right)\left\langle\left[c(t), c^{\dagger}\left(t^{\prime}\right)\right]_{\mp}\right\rangle, \\
G^{K}\left(t, t^{\prime}\right) & =\frac{1}{2}\left(G_{11}+G_{12}+G_{21}+G_{22}\right) \\
& =-i\left\langle\left[c(t), c^{\dagger}\left(t^{\prime}\right)\right]_{ \pm}\right\rangle, \\
G^{\urcorner}\left(t, \tau^{\prime}\right) & =\frac{1}{2}\left(G_{13}+G_{23}\right)=\mp i\left\langle c^{\dagger}\left(\tau^{\prime}\right) c(t)\right\rangle, \\
G^{\ulcorner}\left(\tau, t^{\prime}\right) & =\frac{1}{2}\left(G_{31}+G_{32}\right)=-i\left\langle c(\tau) c^{\dagger}\left(t^{\prime}\right)\right\rangle, \\
G^{M}\left(\tau, \tau^{\prime}\right) & =-i G_{33}=-\left\langle\mathcal{T}_{\tau} c(\tau) c^{\dagger}\left(\tau^{\prime}\right)\right\rangle .
\end{aligned}
$$

In the above formulas, we choose the upper (lower) sign if the operators $c$ and $c^{\dagger}$ are bosonic (fermionic), [, $]_{-(+)}$denotes an (anti-)commutator, $t, t^{\prime} \in \mathcal{C}_{1} \cup \mathcal{C}_{2}, \tau, \tau^{\prime} \in \mathcal{C}_{3}, \theta(t)$ is a step function, and $\mathcal{T}_{\tau}$ is the time-ordering operator on the imaginary-time axis. Note that the anticommutator is used for bosonic operators while the commutator is used for fermionic operators in $G^{K}$ [Eq. (17c)]. For convenience, we also define the lesser and greater Green's functions

$$
\begin{aligned}
& G^{<}\left(t, t^{\prime}\right)=G_{12}=\mp i\left\langle c^{\dagger}\left(t^{\prime}\right) c(t)\right\rangle, \\
& G^{>}\left(t, t^{\prime}\right)=G_{21}=-i\left\langle c(t) c^{\dagger}\left(t^{\prime}\right)\right\rangle,
\end{aligned}
$$

which are related to the retarded, advanced, and Keldysh Green's functions via 


$$
\begin{aligned}
& G^{<}=\frac{1}{2}\left(G^{K}-G^{R}+G^{A}\right), \\
& G^{>}=\frac{1}{2}\left(G^{K}+G^{R}-G^{A}\right) .
\end{aligned}
$$

In addition to the redundancy (15), the components of (14) are related via their Hermitian conjugates. For the physical Green's function components, conjugation yields

$$
\begin{gathered}
G^{<,>, K}\left(t, t^{\prime}\right)^{*}=-G^{<,>, K}\left(t^{\prime}, t\right), \\
G^{R}\left(t, t^{\prime}\right)^{*}=G^{A}\left(t^{\prime}, t\right), \\
G^{\urcorner}\left(t, \tau^{\prime}\right)^{*}=\mp G\left\ulcorner\left(\beta-\tau^{\prime}, t\right),\right.
\end{gathered}
$$

where we take the upper (lower) sign in Eq. (19c) for bosons (fermions). Finally, if a fermionic system has particle-hole symmetry, the Green's function is antisymmetric

$$
G\left(t, t^{\prime}\right)=-G\left(t^{\prime}, t\right)
$$

In addition to these symmetries, it follows from the cyclic invariance of the trace and the definition of $\mathcal{T}_{\mathcal{C}}$ that $G\left(t, t^{\prime}\right)$ satisfies a boundary condition on $\mathcal{C}$ in both arguments,

$$
\begin{aligned}
& G\left(0^{+}, t\right)= \pm G(-i \beta, t), \\
& G\left(t, 0^{+}\right)= \pm G(t,-i \beta),
\end{aligned}
$$

where $0^{+} \in \mathcal{C}_{1}$ and $-i \beta \in \mathcal{C}_{3}$ denote the two end points of $\mathcal{C}$, and the upper (lower) sign corresponds to the case of bosons (fermions).

The Matsubara component $G^{M}$ plays a somewhat special role, since it is always translationally invariant $G^{M}\left(\tau, \tau^{\prime}\right) \equiv$ $G^{M}\left(\tau-\tau^{\prime}\right) \quad(\mathcal{H}$ does not depend on imaginary time $)$. Furthermore, it is real (Hermitian), $G^{M}(\tau)^{*}=G^{M}(\tau)$, and as a consequence of Eq. (21) it is periodic (antiperiodic) for bosons (fermions), $G^{M}(\tau)= \pm G^{M}(\tau+\beta)$. One can thus use its Fourier decomposition in terms of Matsubara frequencies

$$
\begin{gathered}
G^{M}\left(\tau, \tau^{\prime}\right)=T \sum_{n} e^{-i \omega_{n}\left(\tau-\tau^{\prime}\right)} G^{M}\left(i \omega_{n}\right), \\
G^{M}\left(i \omega_{n}\right)=\int_{0}^{\beta} d \tau e^{i \omega_{n} \tau} G^{M}(\tau) .
\end{gathered}
$$

Using the physical Green's function components instead of the full matrix (14) can be quite beneficial in numerical simulations, since with this one almost automatically exploits the symmetries and redundancies and thus reduces the amount of data to be handled (see Sec. II.A.1.e). Moreover, the components (17) are often used to interpret the results of calculations since they have an intuitive interpretation, which originates from their physical meaning in equilibrium: When $\mathcal{H}$ does not depend on time, real-time components of $G$ depend on the time difference only and can be represented via their Fourier transform. The imaginary part of the retarded (or advanced) Green's function gives the single-particle spectral function (Abrikosov, Gorkov, and Dzyaloshinski, 1975)

$$
A(\omega)=-\frac{1}{\pi} \operatorname{Im} G^{R}(\omega)=\frac{1}{\pi} \operatorname{Im} G^{A}(\omega),
$$

which represents the density of single-particle excitations at energy $\omega$ of the many-body state, as can be seen from the Lehmann representation (Mahan, 2000)

$$
A(\omega)=\frac{1}{Z} \sum_{m n}\left(\mp e^{-\beta E_{n}}+e^{-\beta E_{m}}\right)\left|\left\langle n\left|c^{\dagger}\right| m\right\rangle\right|^{2} \delta\left(\omega-E_{n}+E_{m}\right) .
$$

Out of equilibrium, one can still define the spectral function using the partial Fourier transformation

$$
A\left(\omega, t_{\mathrm{av}}\right)=-\frac{1}{\pi} \operatorname{Im} \int d t_{\mathrm{rel}} e^{i \omega t_{\mathrm{rel}}} G^{R}\left(t, t^{\prime}\right)
$$

$\left[t_{\mathrm{av}}=\left(t+t^{\prime}\right) / 2, t_{\mathrm{rel}}=t-t^{\prime}\right]$, which satisfies the sum rule

$$
\int d \omega A\left(\omega, t_{\mathrm{av}}\right)=1
$$

Higher moment sum rules have also been derived (Turkowski and Freericks, 2006, 2008). These relations hold exactly in and out of equilibrium, so that they are quite useful in benchmarking calculations.

In equilibrium, all components of $G$ can be related to the spectral function

$$
G\left(t, t^{\prime}\right)=-i \int d \omega e^{-i \omega\left(t-t^{\prime}\right)} A(\omega)\left[\theta_{\mathcal{C}}\left(t, t^{\prime}\right) \pm f(\omega)\right],
$$

where $f(\omega)=1 /\left(e^{\beta \omega} \mp 1\right)$ is the Bose (Fermi) occupation function. Equation (27) follows from the analytic properties of the Green's function components as a function of the time difference, together with the Kubo-Martin-Schwinger boundary condition (21) (Kubo, 1957; Martin and Schwinger, 1959), and can also be read off a Lehmann representation. In particular, the imaginary part of the lesser (greater) Green's function thus yields the density of occupied (unoccupied) states

$$
\begin{gathered}
\mp \operatorname{Im} G^{<}(\omega)=2 \pi A(\omega) f(\omega) \equiv 2 \pi N(\omega), \\
-\operatorname{Im} G^{>}(\omega)=2 \pi A(\omega)[1 \pm f(\omega)] .
\end{gathered}
$$

In essence, Eq. (28) is the fluctuation-dissipation relation (Kubo, 1957; Mahan, 2000) for single-particle excitations

$$
G^{K}(\omega)=F(\omega)\left[G^{R}(\omega)-G^{A}(\omega)\right],
$$

where

$$
F(\omega)=1 \pm 2 f(\omega)= \begin{cases}\operatorname{coth}\left(\frac{\beta \omega}{2}\right) & \text { for bosons } \\ \tanh \left(\frac{\beta \omega}{2}\right) & \text { for fermions }\end{cases}
$$

In equilibrium, the density of occupied (unoccupied) states is often taken as a first approximation to understand (inverse) photoemission spectroscopy in correlated materials. Similarly, intensities for time-resolved (inverse) photoemission spectroscopy can be obtained from the real-time Green's functions $G^{<}\left(t, t^{\prime}\right)$ and $G^{>}\left(t, t^{\prime}\right)$ (Sec. II.B.5). 
We conclude this section with the remark that all relations concerning contour-ordered Green's functions remain valid if one replaces the time-ordered exponential in Eq. (9) by a more general action, for example,

$$
S=-i \int_{C} d t \mathcal{H}(t)-i \int_{C} d t d t^{\prime} c^{\dagger}(t) \Delta\left(t, t^{\prime}\right) c\left(t^{\prime}\right),
$$

where $\Delta\left(t, t^{\prime}\right)$ is a function on the contour with the same boundary and symmetry properties as the Green's function. In this case, the contour-ordered Green's function is defined as

$$
G\left(t, t^{\prime}\right)=-i\left\langle\mathcal{T}_{c} c(t) c^{\dagger}\left(t^{\prime}\right)\right\rangle_{s},
$$

where the expectation value of observables with respect to $S$ is

$$
\langle\cdots\rangle_{S}=\frac{\operatorname{Tr}\left[\mathcal{T}_{C} \exp (S) \cdots\right]}{\operatorname{Tr}\left[\mathcal{T}_{C} \exp (S)\right]} .
$$

The action (30) arises naturally when parts of a system are traced out in order to derive an effective description of the rest. Expressions like Eq. (30) can conveniently be rephrased in terms of path integrals over Grassmann variables (Negele and Orland, 1988; Kamenev, 2011), but throughout this review we stay with the equivalent formulation in terms of time-ordered expectation values.

\section{c. Noninteracting contour-ordered Green's functions}

Here we discuss equations of motion for noninteracting Green's functions. For a tight-binding model $\mathcal{H}_{0}(t)=$ $\sum_{k}\left[\epsilon_{k}(t)-\mu\right] c_{k}^{\dagger} c_{k}$ one can directly compute the time derivatives of $G_{0, k}\left(t, t^{\prime}\right)=-i\left\langle\mathcal{T}_{c} c_{k}(t) c_{k}^{\dagger}\left(t^{\prime}\right)\right\rangle$ :

$$
\begin{array}{r}
{\left[i \partial_{t}+\mu-\epsilon_{k}(t)\right] G_{0, k}\left(t, t^{\prime}\right)=\delta_{\mathcal{C}}\left(t, t^{\prime}\right),} \\
G_{0, k}\left(t, t^{\prime}\right)\left[-i \overleftarrow{\partial}_{t^{\prime}}+\mu-\epsilon_{k}\left(t^{\prime}\right)\right]=\delta_{\mathcal{C}}\left(t, t^{\prime}\right),
\end{array}
$$

where we used the notation for contour calculus introduced in Table I $\left[f(t) \overleftarrow{\partial}_{t} \equiv \partial_{t} f(t)\right.$ is acting to the left]. The two equations are equivalent, and each determines $G_{0, k}$ uniquely if solved with the boundary condition (21) (Turkowski and Freericks, 2005):

$$
G_{0, \boldsymbol{k}}\left(t, t^{\prime}\right)=-i\left[\theta_{\mathcal{C}}\left(t, t^{\prime}\right) \pm f\left(\epsilon_{\boldsymbol{k}}(0)-\mu\right)\right] e^{-i \int_{t^{\prime}}^{t} d \bar{t}\left[\epsilon_{\boldsymbol{k}}(\bar{t})-\mu\right]} .
$$

The two equations of motion (33) can be rephrased by introducing the inverse of the Green's function

TABLE I. Notation for the contour calculus used in this text: (2) and (3): contour integration and convolution; (4): time derivative (not a derivative along the contour); (5) and (6): contour theta and delta functions.

(1) $t^{ \pm}, t \in\left[0, t_{\max }\right]$ : point on $\mathcal{C}_{1,2} ;-i \tau, \tau \in[0, \beta]$ : point on $\mathcal{C}_{3}$

(2) $\quad \int_{C} d t g(t)=\int_{0}^{t_{\max }} d t g\left(t^{+}\right)-\int_{0}^{t_{\max }} d t g\left(t^{-}\right)-i \int_{0}^{\beta} d \tau g(-i \tau)$

(3) $\quad[a * b]\left(t, t^{\prime}\right)=\int_{\mathcal{C}} d \bar{t} a(t, \bar{t}) b\left(\bar{t}, t^{\prime}\right)$

$$
\partial_{t} g(t)= \begin{cases}\partial_{t} g\left(t^{ \pm}\right) & t \in \mathcal{C}_{1,2} \\ i \partial_{\tau} g(-i \tau) & t=-i \tau \in \mathcal{C}_{3}\end{cases}
$$$$
\theta_{\mathcal{C}}\left(t, t^{\prime}\right)= \begin{cases}1 & t \succ t^{\prime} \\ 0 & \text { else }\end{cases}
$$

(6) $\quad \delta_{C}\left(t, t^{\prime}\right)=\partial_{t} \theta_{C}\left(t, t^{\prime}\right), \int_{C} d \bar{t} \delta_{C}(t, \bar{t}) g(\bar{t})=g(t) \forall g(t)$

$$
G_{0, k}^{-1}\left(t, t^{\prime}\right)=\left[i \partial_{t}+\mu-\epsilon_{k}(t)\right] \delta_{C}\left(t, t^{\prime}\right),
$$

which is a differential operator on the contour. Equations (33) then simply read $G_{0, k}^{-1} * G_{0, k}=G_{0, k} * G_{0, k}^{-1}=\delta_{\mathcal{C}}$, where the star $(*)$ denotes a convolution (Table I). Closed equations of motion can also be derived for the general case in which the action is nonlocal in time [cf. Eq. (30)], and $\mathcal{H}_{0}$ is not diagonal in orbitals

$$
\mathcal{S}=-i \int_{\mathcal{C}} d t \mathcal{H}_{0}(t)-i \sum_{i j} \int_{\mathcal{C}} d t d t^{\prime} c_{i}^{\dagger}(t) \Delta_{i j}\left(t, t^{\prime}\right) c_{j}\left(t^{\prime}\right)
$$

with $\mathcal{H}_{0}(t)=\sum_{i j}\left[v_{i j}(t)-\mu \delta_{i j}\right] c_{i}^{\dagger} c_{j}$. In this case, both $G_{0}$ and $G_{0}^{-1}$ are matrices in orbital indices, and

$$
\left(G_{0}^{-1}\right)_{i j}\left(t, t^{\prime}\right)=\left[\delta_{i j}\left(i \partial_{t}+\mu\right)-v_{i j}(t)\right] \delta_{C}\left(t, t^{\prime}\right)-\Delta_{i j}\left(t, t^{\prime}\right) .
$$

For $\Delta \neq 0$, the solution of the equation of motion $G_{0}^{-1} * G_{0}=$ $\delta_{C}$ in general requires a numerical technique, which will be discussed in Sec. II.A.1.e.

\section{d. Dyson equation}

To describe nonequilibrium correlated systems using Green's functions, one has to take account of self-energy corrections $\Sigma$ to the noninteracting Green's function $G_{0}$. In the language of Feynman diagrams, the self-energy is the sum of all one-particle irreducible diagrams of the interacting Green's function $G$, i.e., diagrams that cannot be separated into two parts by cutting single $G_{0}$ lines. (The diagram rules are the same for imaginary-time-ordered and contour-ordered Green's functions when imaginary-time integrals over internal vertices are replaced by contour integrals.) The self-energy is defined on the contour $\mathcal{C}$ (Fig. 4), so that it satisfies symmetry and boundary conditions analogous to that of the Green's functions (Sec. II.A.1.b). The fully interacting Green's function $G=G_{0}+G_{0} * \Sigma * G_{0}+G_{0} * \Sigma * G_{0} * \Sigma * G_{0}+\cdots$ is then given by the Dyson equation

$$
\begin{aligned}
G & =G_{0}+G_{0} * \Sigma * G \\
& =G_{0}+G * \Sigma * G_{0} .
\end{aligned}
$$

To evaluate the self-energy is truly a nonequilibrium quantum many-body problem, and one generally needs additional techniques, which will be explained in the following sections. Once the self-energy is fixed, the full Green's function is determined from one of the two equivalent integral equations (38), which is still a formidable numerical task that will be discussed in the remainder of this section.

We can transform the Dyson equation and its conjugate from its integral form into a differential form by convoluting with the operator $G_{0}^{-1}$ from the left [Eq. (38a)], or right [Eq. (38b)], respectively,

$$
\left[G_{0}^{-1}-\Sigma\right] * G=G *\left[G_{0}^{-1}-\Sigma\right]=\delta_{C} .
$$

The result is conveniently expressed by the definition $G^{-1}=G_{0}^{-1}-\Sigma$. In this abstract notation, the Dyson equation 
is identical to the form used in equilibrium (Mahan, 2000). However, the same equation (39) has conceptually a very different meaning for Matsubara and contour-ordered Green's functions. Using a differential form for $G_{0}^{-1}$ analogous to Eq. (35), one can see that the two Eqs. (39) are integraldifferential equations of the generic form

$$
\begin{gathered}
{\left[i \partial_{t}-h(t)\right] G\left(t, t^{\prime}\right)-\int_{\mathcal{C}} d \bar{t} \Sigma(t, \bar{t}) G\left(\bar{t}, t^{\prime}\right)=\delta_{\mathcal{C}}\left(t, t^{\prime}\right),} \\
G\left(t, t^{\prime}\right)\left[-i \overleftarrow{\partial}_{t^{\prime}}-h\left(t^{\prime}\right)\right]-\int_{\mathcal{C}} d \bar{t} G(t, \bar{t}) \Sigma\left(\bar{t}, t^{\prime}\right)=\delta_{C}\left(t, t^{\prime}\right) .
\end{gathered}
$$

The time derivative $\partial_{t} G$ in these equations is related to the value of $G$ at different times via the convolution $\Sigma * G$. Equations (40) are causal, and thus provide a non-Markovian time-propagation scheme for $G$, in which the self-energy takes the role of a memory kernel (Sec. II.A.1.e). On the imaginary branch, on the other hand, the same equations provide a boundary-value problem for the (Matsubara) Green's functions of an equilibrium state (which play the role of an initial value for the time propagation). The solution of the integraldifferential equation (40) has numerous applications in various areas of physics, including condensed-matter physics, nuclear physics, high-energy physics, and cosmology (Bonitz, 2000; Bonitz and Semkat, 2003; Bonitz and Filinov, 2006; Bonitz and Balzer, 2010). The biggest challenge is to deal with the memory effects in a proper way. Traditionally one tries to reduce the memory depth by deriving quantum Boltzmann equations (Kadanoff and Baym, 1962; Mahan, 1984; Rammer, 1998; Haug and Jauho, 2008), or by using decoupling schemes like the generalized Kadanoff-Baym ansatz (Lipavský, Špička, and Velický, 1986). While those approaches usually work well for weakly interacting systems or in the semiclassical limit, one must account for the full memory when dealing with the ultrafast time evolution in strongly correlated systems.

\section{e. Numerical solution}

In this section, we discuss the numerical solution of the generic contour equation (40). On a suitable time grid, with $N$ time slices $\Delta t=t_{\max } / N$ on $C_{1,2}$ and $M$ imaginary time slices $\Delta \tau=\beta / M$ on $C_{3}$, the operator $\left[i \partial_{t}-h(t)\right] \delta_{C}\left(t, t^{\prime}\right)-\Sigma\left(t, t^{\prime}\right)$ can be written as a $(2 N+M+1)$-dimensional matrix [with some care to correctly discretize the singular operators $\delta_{\mathcal{C}}\left(t, t^{\prime}\right)$ and $\left.\partial_{t} \delta_{C}\left(t, t^{\prime}\right)\right]$, such that the solution for $G$ becomes a matrix inversion (Freericks, 2008). On the other hand, there is a slightly tedious but rather powerful approach which is based on an equivalent set of integral-differential equations for the physical components [Eq. (17)] of $G$, known as KadanoffBaym equations (Kadanoff and Baym, 1962; Bonitz, 2000). This approach, which has been introduced to nonequilibrium DMFT by Tran (2008), interprets Eq. (40) as a non-Markovian time-propagation scheme, which can be of great value both conceptually and numerically, and it automatically exploits the symmetries (15) and (19).

For this procedure we choose a subset of the components (17) that completely parametrize $G$, taken into account the symmetries (15) and (19), e.g., $\left.G^{M}, G^{R}, G\right\urcorner$, and $G^{<}$. Using their definition (17) and the definition of the convolution (Table I), one can express the physical components of a convolution $\Sigma * G$ in terms of the components of $G$ and $\Sigma$ using the Langreth rules (Langreth, 1976), and hence derive four coupled integral equations

$$
\begin{aligned}
& {\left[-\partial_{\tau}-h\left(0^{-}\right)\right] G^{M}(\tau)-\int_{0}^{\beta} d \bar{\tau} \Sigma^{M}(\tau-\bar{\tau}) G^{M}(\bar{\tau})=\delta(\tau),} \\
& {\left[i \partial_{t}-h(t)\right] G^{R}\left(t, t^{\prime}\right)-\int_{t^{\prime}}^{t} d \bar{t} \Sigma^{R}(t, \bar{t}) G^{R}\left(\bar{t}, t^{\prime}\right)=\delta\left(t-t^{\prime}\right), \quad \text { (41) }} \\
& {\left[i \partial_{t}-h(t)\right] G^{\urcorner}\left(t, \tau^{\prime}\right)-\int_{0}^{t} d \bar{t} \Sigma^{R}(t, \bar{t}) G^{\urcorner}\left(\bar{t}, \tau^{\prime}\right)=Q^{\urcorner}\left(t, \tau^{\prime}\right),} \\
& {\left[i \partial_{t}-h(t)\right] G^{<}\left(t, t^{\prime}\right)-\int_{0}^{t} d \bar{t} \Sigma^{R}(t, \bar{t}) G^{<}\left(\bar{t}, t^{\prime}\right)=Q^{<}\left(t, t^{\prime}\right),}
\end{aligned}
$$

with

$$
\begin{aligned}
Q^{\urcorner}\left(t, \tau^{\prime}\right)= & \int_{0}^{\beta} d \bar{\tau} \Sigma^{\prime}(t, \bar{\tau}) G^{M}\left(\bar{\tau}, \tau^{\prime}\right), \\
Q^{<}\left(t, t^{\prime}\right)= & \int_{0}^{t^{\prime}} d \bar{t} \Sigma^{<}(t, \bar{t}) G^{A}\left(\bar{t}, t^{\prime}\right) \\
& -i \int_{0}^{\beta} d \bar{\tau} \Sigma^{\urcorner}(t, \bar{\tau}) G^{\ulcorner}\left(\bar{\tau}, t^{\prime}\right) .
\end{aligned}
$$

Here the integral limits take into account that retarded functions vanish for $t<t^{\prime}$. Together with the boundary condition (21) these Kadanoff-Baym equations determine $G$ uniquely. To see how these Kadanoff-Baym equations represent the above mentioned time-propagation scheme, one may first notice that Eq. (41a) for $G^{M}$ is decoupled from the rest: It must be solved with the boundary condition $G^{M}(\tau)=$ $\pm G^{M}(\tau+\beta)$ for bosons ( + ) or fermions (-). Hence one can solve it by Fourier transformation (22), and its solution is the Green's function of the initial equilibrium state

$$
G^{M}\left(i \omega_{n}\right)=\left[i \omega_{n}-h\left(0^{-}\right)-\Sigma^{M}\left(i \omega_{n}\right)\right]^{-1},
$$

independent of the subsequent perturbation of the system. The remaining equations (41b)-(41d) have an inherent causal structure: If $G_{t}=\left\{G^{R}\left(t, t^{\prime}\right), G^{\urcorner}(t, \tau), G^{<}\left(t, t^{\prime}\right) \mid 0 \leq \tau \leq \beta\right.$, $\left.t^{\prime} \leq t\right\}$ denotes the values of $G$ at "time slice $t$," then $\partial_{t} G_{t}$ is determined by $G^{M}$ (the initial state), $\Sigma_{t}$, and $G_{t^{\prime}}$ for $t^{\prime} \leq t$ (Fig. 5). One can therefore always solve (41b)-(41d) by successively increasing $t$. Furthermore, when one keeps the second time argument of $G$ fixed in Eqs. (41b)-(41d) one obtains a set of one-dimensional integral-differential equations of the type

$$
\frac{d}{d s} y(s)=q(s)+p(s) y(s)+\int_{0}^{s} d \bar{s} k(s, \bar{s}) y(\bar{s}),
$$

i.e., Volterra equations of the second kind (Brunner and van der Houwen, 1986). The causal structure of this equation 


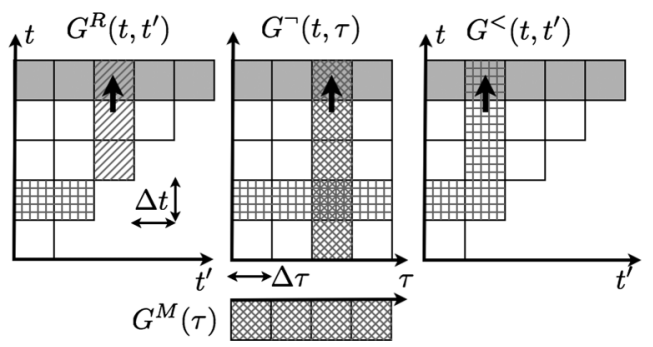

FIG. 5. Causal structure of the Kadanoff-Baym equation (41), when $G$ is computed on one time slice of an equally spaced grid (shaded area): The computation of derivatives for the propagation from the previous time slice (bold arrows) addresses $G$ only at earlier times, marked by a diagonal pattern for $\partial_{t} G^{R}$, diamond pattern for $\left.\partial_{t} G\right\urcorner$, and checkerboard pattern for $\partial_{t} G^{<}$. Hermitian symmetries (19) are used to relate $G^{A}$ with $G^{R}, G^{<}\left(t, t^{\prime}\right)$ with $G^{<}\left(t^{\prime}, t\right)$ and $G\left\ulcorner\right.$ with $G^{\urcorner}$.

is evident from the limits of the integral. For example, for Eq. (41b) we define $y(s)=G^{R}(t+s, t)$, and $k(s, \bar{s})=$ $\Sigma^{R}(t+s, t+s-\bar{s})$, and the initial condition is provided by $y(s<0)=0$. In practice, one thus solves a large number of coupled Volterra equations, for which very stable and accurate high-order algorithms can be found in the literature (Linz, 1985; Brunner and van der Houwen, 1986; Press et al., 1992). In Appendix A, we show one of them, namely, the implicit Runge-Kutta method or the collocation method.

Detailed descriptions of the numerical implementation of the Kadanoff-Baym equations can be found in Köhler, Kwong, and Yousif (1999), Stan, Dahlen, and van Leeuwen (2009), Eckstein, Kollar, and Werner (2010), and Balzer and Bonitz (2013). In general, the required resources scale like $\mathcal{O}\left(M^{2}\right)$ for memory and $\mathcal{O}\left(M^{3}\right)$ for CPU time, where $M$ is the number of time-discretization steps. In particular the memory can be a limiting factor, when Green's functions carry many orbital indices, such that efficient shared memory or even (distributed-memory) parallelization schemes can become necessary (Balzer and Bonitz, 2013). To validate the accuracy of the numerics, checking nonequilibrium sum rules (Turkowski and Freericks, 2006, 2008) can be very helpful.

We conclude this section by mentioning two other generic contour equations, which appear frequently in nonequilibrium DMFT. These are

$$
\begin{gathered}
{[1+F] * G=Q,} \\
F * G=Q,
\end{gathered}
$$

to be solved for $G$, where $Q$ and $F$ are contour Green's functions. Both equations have a causal structure analogous to Eq. (40). Their numerical solution differs only in that there is no derivative term, and the existence of the source term on the right-hand side. Hence an analogous time-propagation scheme exists (Eckstein, Kollar, and Werner, 2010), in which Eqs. (44) and (45) are reduced to Volterra integral equations of the second and first kind, respectively (Brunner and van der Houwen, 1986),

$$
\begin{gathered}
y(s)+\int_{0}^{s} d \bar{s} k(s, \bar{s}) y(\bar{s})=q(s), \\
\int_{0}^{s} d \bar{s} k(s, \bar{s}) y(\bar{s})=q(s) .
\end{gathered}
$$

At this point, it may be interesting to note that in general highorder accurate propagation schemes for Volterra equations of the first kind are unstable (Brunner and van der Houwen, 1986; Press et al., 1992). Fortunately, within nonequilibrium DMFT one can always rewrite the equations in order to take advantage of the "stabilizing 1" in Eq. (44).

\section{Keldysh formalism for nonequilibrium steady states}

\section{a. Keldysh formalism}

An alternative and simpler approach to nonequilibrium Green's functions is the Keldysh formalism (Keldysh, 1964), which is particularly well suited to describe nonequilibrium steady states of open systems, in which the energy supplied to the system by an external driving field is balanced by the energy flowing out to the environment. In the original Keldysh theory, it is assumed that the initial state is noninteracting, and an interaction is adiabatically turned on from $t=-\infty$. Since no interaction vertex can be inserted on the imaginary-time axis, correlations between the initial state and the timeevolving state represented by the mixed self-energy $\Sigma\urcorner$ and $\Sigma\ulcorner$ (initial correlations) vanish. The imaginary-time contour $C_{3}$ is thus decoupled from $C_{1}$ and $C_{2}$, and one can restrict oneself to the real-time branches $\mathcal{C}_{K}=\mathcal{C}_{1} \cup \mathcal{C}_{2}$ (Keldysh contour, Fig. 6). The contour-ordered Green's function is closed on a $2 \times 2$ subspace of (14):

$$
\hat{G}=\left(\begin{array}{ll}
G^{11} & G^{12} \\
G^{21} & G^{22}
\end{array}\right)
$$

One can conveniently carry out the transformation to physical Green's functions (17) by introducing the matrices

$$
L=\frac{1}{\sqrt{2}}\left(\begin{array}{cc}
1 & -1 \\
1 & 1
\end{array}\right), \quad \tau_{3}=\left(\begin{array}{cc}
1 & 0 \\
0 & -1
\end{array}\right) .
$$

$L$ is a unitary matrix, while $\tau_{3}$ represents the sign of the measure $d t$ that appears in an integral along $\mathcal{C}_{K}$. Using these, we perform a linear transformation [Keldysh rotation (Keldysh, 1964; Larkin and Ovchinnikov, 1975; Rammer, 1998)], obtaining

$$
\underline{G} \equiv L \tau_{3} \hat{G} L^{\dagger}=\left(\begin{array}{cc}
G^{R} & G^{K} \\
0 & G^{A}
\end{array}\right)
$$

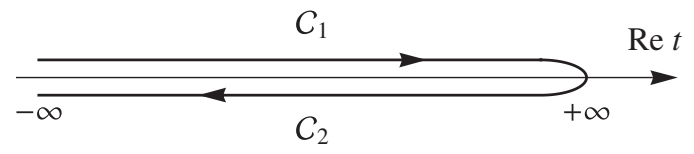

FIG. 6. The Keldysh contour $\mathcal{C}_{K}=\mathcal{C}_{1} \cup \mathcal{C}_{2}$ with the two branches ranging from $-\infty$ to $\infty$. 
Convolutions along $\mathcal{C}_{K}$ are then simply written as onedimensional time integrals, $\underline{A * B}\left(t, t^{\prime}\right)=\int_{-\infty}^{\infty} d \bar{t} \underline{A}(t, \bar{t}) \underline{B}\left(\bar{t}, t^{\prime}\right)$.

In general, initial correlations are supposed to be relevant in a realistic situation, since the interaction always exists in the initial state. However, in a dissipative system coupled to an external heat bath the initial correlation is expected to disappear in the long-time limit, since the large number of degrees of freedom in the heat bath would influence the longtime dynamics, and wipe out the information of the initial state and initial transient dynamics. In this case, the Keldysh formalism is applicable to the nonequilibrium steady state without the use of adiabatic switching of interactions. Although the independence on the initial state is assumed to be true in general, it is extremely hard to prove this fact rigorously for a given model, as it is ultimately related to the fundamental question of thermalization of the system (Polkovnikov et al., 2011; see also Sec. III.B).

\section{b. Free-fermion bath}

To describe a nonequilibrium steady state in a dissipative system, one may consider a system coupled to an environment (open system)

$$
H_{\text {tot }}=H_{s}+H_{\text {mix }}+H_{\text {bath }} .
$$

Here $H_{s}$ and $H_{\text {bath }}$ are Hamiltonians of the system and environment, respectively, and $H_{\text {mix }}$ represents a coupling between them. $H_{\text {bath }}$ is assumed to have a much larger number of degrees of freedom than $H_{s}$, so that $H_{\text {bath }}$ acts as a heat bath for the system. When the system is excited by an external field, the energy injected from the field flows to the bath, resulting in the energy dissipation.

The simplest model of the heat bath, that can be solved analytically, is a free-fermion bath (Tsuji, Oka, and Aoki, 2009; Amaricci et al., 2012; Aron, 2012; Werner and Eckstein, 2012; Han, 2013) defined by

$$
\begin{aligned}
H_{\text {mix }} & =\sum_{i, p} V_{p}\left(c_{i}^{\dagger} b_{i, p}+b_{i, p}^{\dagger} c_{i}\right), \\
H_{\text {bath }} & =\sum_{i, p}\left(\epsilon_{b, p}-\mu_{b}\right) b_{i, p}^{\dagger} b_{i, p},
\end{aligned}
$$

where $c_{i}^{\dagger}\left(c_{i}\right)$ creates (annihilates) the system's fermions, $b_{i, p}^{\dagger}$ $\left(b_{i, p}\right)$ creates (annihilates) fermionic degrees of freedom of the bath, $\epsilon_{b, p}$ is the bath level energy, and $V_{p}$ is the hybridization between the system and the mode $p$ of the bath. The thermal bath is assumed to be equilibrated with temperature $T$. The chemical potential of the bath $\left(\mu_{b}\right)$ is determined such that no current flows between the bath and the system. This model is equivalent to the one where an electrode is attached to every site of the system's lattice, such that the fermions can hop between the lattice and the electrodes (Fig. 7). In other words, the model can be seen as a set of coupled quantum dots connected to independent electrodes [cf. the Büttiker model (Büttiker, 1985, 1986)]. This is one explicit realization of a grand canonical ensemble, in which the system is coupled to a particle reservoir.

The bath's degrees of freedom can be analytically integrated out (Feynman and Vernon, 1963; Caldeira and Leggett, 1981),

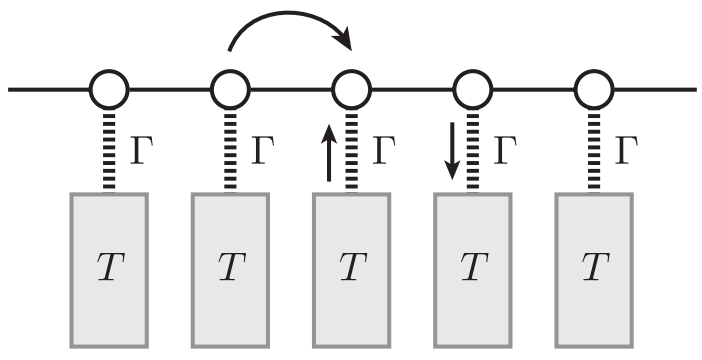

FIG. 7. Schematic representation of a free-fermion bath model. Each site of the system is connected to an electrode with a coupling $\Gamma$ at temperature $T$. The arrows indicate the motion of particles.

since $H_{\text {mix }}+H_{\text {bath }}$ is quadratic in $b_{i, p}^{\dagger}$ and $b_{i, p}$. To this end, we introduce the contour-ordered Green's function of the bath

$$
G_{\mathrm{bath}}\left(p ; t, t^{\prime}\right)=-i\left\langle\mathcal{T}_{\mathcal{C}_{K}} b_{i, p}(t) b_{i, p}^{\dagger}\left(t^{\prime}\right)\right\rangle_{\mathrm{bath}} .
$$

Since the bath's fermions are noninteracting, $G_{\text {bath }}$ can be evaluated exactly. After integrating out $b_{i, p}^{\dagger}$ and $b_{i, p}$, one can show that the effect of the bath is an additional self-energy correction to the system Green's function,

$$
\Sigma_{\text {bath }}\left(t, t^{\prime}\right)=\sum_{p} V_{p} G_{\text {bath }}\left(p ; t, t^{\prime}\right) V_{p},
$$

which is local in space (or does not have momentum dependence in $\boldsymbol{k}$ space). As a result, the Dyson equation for the system Green's function can be symbolically written as

$$
\underline{G}=\left[\underline{G}_{0}^{-1}-\underline{\Sigma}_{\text {bath }}-\underline{\Sigma}\right]^{-1}
$$

in the Keldysh formulation. Since the bath Green's function has a time translation invariance, it is convenient to represent the self-energy $\Sigma_{\text {bath }}$ in real frequency space. The retarded component of $\Sigma_{\text {bath }}$ is

$$
\Sigma_{\text {bath }}^{R}(\omega)=\sum_{p} \frac{V_{p}^{2}}{\omega+\mu_{b}-\epsilon_{b, p}+i \eta}
$$

where $\eta$ is a positive infinitesimal. By using $1 /(\omega+i \eta)=$ $\mathcal{P}(1 / \omega)-i \pi \delta(\omega)$ (with $\mathcal{P}$ the principal value), one can divide $\Sigma_{\text {bath }}^{R}(\omega)$ into real and imaginary parts. The imaginary part defines a spectral function of the heat bath

$$
\Gamma(\omega)=\sum_{p} \pi V_{p}^{2} \delta\left(\omega+\mu_{b}-\epsilon_{b, p}\right) .
$$

A simple treatment of dissipation is to omit the $\omega$ dependence of $\Sigma_{\text {bath }}^{R}(\omega)$. This corresponds to considering a flat density of states $[\Gamma(\omega)=\Gamma]$ for the reservoir. The presence of the imaginary part of $\Sigma_{\text {bath }}^{R}$ is a manifestation of irreversibility, i.e., dissipation of energy (and particles) via the fermionic bath. The real part of $\Sigma_{\text {bath }}^{R}$, a potential shift due to the coupling to the bath, can be absorbed into the chemical potential $\mu$ of the system, so that only the imaginary part affects the dynamics of the system. Recall that the bath is always in equilibrium with temperature $T=\beta^{-1}$. Hence the 
fluctuation-dissipation relation (29a) holds for the bath Green's function, which determines the Keldysh component of the dissipation term. Thus, one has

$$
\left(\begin{array}{cc}
\Sigma_{\text {bath }}^{R}(\omega) & \Sigma_{\text {bath }}^{K}(\omega) \\
0 & \Sigma_{\text {bath }}^{A}(\omega)
\end{array}\right)=\left(\begin{array}{cc}
-i \Gamma & -2 i \Gamma F(\omega) \\
0 & i \Gamma
\end{array}\right),
$$

with $F(\omega)=\tanh (\beta \omega / 2)$ [Eq. (29b)]. The retarded and advanced components are damping terms that cause a relaxation, while the Keldysh component represents thermal fluctuations from the heat bath. Although the model defined by Eqs. (52) and (53) might look somewhat artificial, one can consider it as a phenomenological treatment of dissipation with two parameters $\Gamma$ and $T$, where the damping rate is simply parametrized by a constant $\Gamma$, and the heat-bath temperature by $T$ [analogous to a relaxation time approximation in the classical Boltzmann equation (Mahan, 2000)].

The Keldysh component of $\Sigma_{\text {bath }}$ in Eq. (56) works as a boundary condition and allows one to determine a distribution function for the nonequilibrium steady state. If it is absent, the Keldysh Green's function reads $G^{K}=G^{R}\left[-\left(G_{0}^{-1}\right)^{K}+\Sigma^{K}\right] G^{A}$, where $\left(G_{0}^{-1}\right)^{K} \equiv-G_{0}^{R-1} G_{0}^{K} G_{0}^{A-1}$ contains information about the initial condition. In general, $\left(G_{0}^{-1}\right)^{K}$ is proportional to i $\eta$ in frequency space, and vanishes in the limit of $\eta \rightarrow 0$ (since the noninteracting system is dissipationless). Thus one ends up with $G^{K}=G^{R} \Sigma^{K} G^{A}$ (Keldysh equation), which is, however, homogeneous in the sense that it does not have a source term to determine the Keldysh component of the Green's function. Because of the lack of an input for the distribution function, it is impossible to find a unique solution for the distribution. On the other hand, in the Dyson equation (56) we have a nonzero source term $\Sigma_{\text {bath }}^{K}(\omega)=-2 i \Gamma F(\omega)$ in the Keldysh component, which acts as a boundary condition. Then we can totally neglect the noninteracting term $\left(G_{0}^{-1}\right)^{K}$, i.e., the initial condition is wiped out by dissipation. Equation (56) becomes an inhomogeneous equation, enabling one to determine the nonequilibrium steady state from the boundary condition. This mechanism allows a description of nonequilibrium steady states in dissipative systems within the Keldysh formalism

\section{B. Nonequilibrium dynamical mean-field theory}

\section{Overview of equilibrium DMFT}

Many of the ideas underlying nonequilibrium DMFT are direct generalizations of the conventional equilibrium DMFT formalism (Georges et al., 1996), so it is worthwhile to start this section with a brief overview of equilibrium DMFT and its foundations. Static mean-field theories, such as the Weiss mean-field theory for a spin system $H=\sum_{i j} J_{i j} S_{i} \cdot S_{j}+$ $\sum_{i} \boldsymbol{h}_{i} \cdot \boldsymbol{S}_{i}$, have been known for a long time. In the latter, the spin $\langle\boldsymbol{S}\rangle$ at a given site $i$ is determined by the (thermal) average $\operatorname{Tr}\left[e^{-\beta H_{\text {eff }}} \boldsymbol{S}\right] / Z$, taken with an effective single-site Hamiltonian $H_{\text {eff }}=\left(\boldsymbol{h}_{i}+\boldsymbol{h}_{\mathrm{mf}}\right) \cdot \boldsymbol{S}$ which describes one spin in the average (Weiss) field $\boldsymbol{h}_{\mathrm{mf}}=\sum_{j} J_{i j}\left\langle\boldsymbol{S}_{j}\right\rangle$ due to the interaction with its neighbors. For interacting electrons on a lattice, the simplest static mean-field theory is the Hartree approach, which approximates the Coulomb interaction between the particles by an averaged time-independent potential. Electrons can thus avoid each other only by forming a static long-range order, which is clearly not the true story: Interacting electrons correlate their motion in time, so that they almost never occupy the same orbital simultaneously. A theory of Mott insulators and correlated metals (in particular, the paramagnetic state) must necessarily keep track of these nontrivial time-dependent correlations. DMFT can achieve this goal because it is not an effective theory for the electron density, but for the local frequency-dependent Green's function $G(\omega)$, which contains the information about these timedependent fluctuations. Besides this important difference, a formal analogy to the static mean-field theory remains: $G(\omega)$ is obtained from an effective model that involves only one site of the lattice. This site is coupled to a "fluctuating Weiss field" $\Delta(\omega)$ that resembles the exchange of particles with the rest of the lattice and must be determined self-consistently as a functional of $G$.

In the following we first state and then discuss the equilibrium DMFT equations for the case of a single-band Hubbard model (generalizations are discussed below)

$$
\begin{gathered}
H=\sum_{\langle i j\rangle, \sigma} v_{i j} c_{i \sigma}^{\dagger} c_{j \sigma}+\sum_{i} H_{\mathrm{loc}}^{(i)}, \\
H_{\mathrm{loc}}^{(i)}=U\left(c_{i \uparrow}^{\dagger} c_{i \uparrow}-\frac{1}{2}\right)\left(c_{i \downarrow}^{\dagger} c_{i \downarrow}-\frac{1}{2}\right) .
\end{gathered}
$$

Here $c_{i \sigma}^{\dagger}\left(c_{i \sigma}\right)$ creates (annihilates) an electron with spin $\sigma$ in a Wannier orbital at site $i$ of a crystal lattice, $v_{i j}$ is the hopping matrix element (we use the symbol $v$ in order to avoid later confusion with time $t$ ), and electrons interact via a local Coulomb interaction $U$. The key approximation of DMFT is that the electronic self-energy is taken to be local in space

$$
\Sigma_{i j}\left(i \omega_{n}\right)=\delta_{i j} \Sigma_{i i}\left(i \omega_{n}\right)
$$

where $\sigma$ is suppressed for simplicity. Furthermore, it is assumed that $\Sigma_{i i}\left(i \omega_{n}\right)$ and the local Green's function $G_{i i}(\tau)=$ $-\left\langle\mathcal{T}_{\tau} c_{i \sigma}(\tau) c_{i \sigma}^{\dagger}(0)\right\rangle=T \sum_{n} e^{-i \omega_{n} \tau} G\left(i \omega_{n}\right)$ can be computed from an effective impurity model with action (Georges and Kotliar, 1992)

$$
\mathcal{S}_{i}=-\int_{0}^{\beta} d \tau H_{\mathrm{loc}}(\tau)-\int_{0}^{\beta} d \tau d \tau^{\prime} \sum_{\sigma} c_{\sigma}^{\dagger}(\tau) \Delta_{i}\left(\tau-\tau^{\prime}\right) c_{\sigma}\left(\tau^{\prime}\right)
$$

where $\Delta$ is the hybridization to a fictitious bath. One has

$$
\begin{gathered}
G_{i i}(\tau)=-\operatorname{Tr}\left[\mathcal{T}_{\tau} e^{S_{i}} c(\tau) c^{\dagger}(0)\right] / Z, \\
G_{i i}\left(i \omega_{n}\right)^{-1}=i \omega_{n}+\mu-\Delta_{i}\left(i \omega_{n}\right)-\Sigma_{i i}\left(i \omega_{n}\right),
\end{gathered}
$$

where Eq. (65) is the Dyson equation for the impurity model that defines the self-energy $\Sigma_{i i}\left(i \omega_{n}\right)$. Because $\Sigma_{i i}$ and $G_{i i}$ are related by the Dyson equation of the lattice model

$$
G_{i j}^{-1}\left(i \omega_{n}\right)=\delta_{i j}\left[i \omega_{n}+\mu-\Sigma_{i i}\left(i \omega_{n}\right)\right]-v_{i j}
$$

the auxiliary quantity $\Delta$ can be eliminated to close the equations. The hybridization function $\Delta(\omega)$ plays the role 
of a (frequency-dependent) Weiss field, and Eqs. (65) and (66) provide the (implicit) functional relation between $G(\omega)$ and $\Delta(\omega)$. Closed relations $\Delta[G]$ can be obtained, for example, for hopping models on the Bethe lattice, in particular, for nearestneighbor hopping $v_{*} / \sqrt{z}$ with coordination number $z \rightarrow \infty$, which gives (Georges et al., 1996)

$$
\Delta\left(i \omega_{n}\right)=v_{*}^{2} G\left(i \omega_{n}\right) .
$$

For a translationally invariant system, with $\Sigma_{i i}\left(i \omega_{n}\right) \equiv$ $\Sigma\left(i \omega_{n}\right)$, Eq. (66) can be solved for $G_{i i}$ in the form

$$
\begin{aligned}
G_{i i}\left(i \omega_{n}\right) & =\frac{1}{L} \sum_{k} G_{k}\left(i \omega_{n}\right)=\frac{1}{L} \sum_{k} \frac{1}{i \omega_{n}+\mu-\Sigma\left(i \omega_{n}\right)-\epsilon_{k}} \\
& =\int d \epsilon \frac{D(\epsilon)}{i \omega_{n}+\mu-\Sigma\left(i \omega_{n}\right)-\epsilon}
\end{aligned}
$$

where $L$ is the number of lattice sites, $G_{k}(\tau)=$ $-\left\langle\mathcal{T}_{\tau} c_{k \sigma}(\tau) c_{\boldsymbol{k} \sigma}^{\dagger}(0)\right\rangle$ is the momentum-resolved Green's function, and $D(\epsilon)=1 / L \sum_{k} \delta\left(\epsilon-\epsilon_{k}\right)$ is the local density of states. An important case is the semielliptic density of states

$$
D(\epsilon)=\frac{1}{2 \pi v_{*}^{2}} \sqrt{4 v_{*}^{2}-\epsilon^{2}},
$$

which corresponds to nearest-neighbor hopping on the Bethe lattice with infinite coordination number and thus implies Eq. (67).

The starting point for the derivation of the DMFT equations (62)-(66) has been the limit of infinite dimensions (Metzner and Vollhardt, 1989). A meaningful limit $d \rightarrow \infty$ is obtained when the hopping matrix elements are rescaled such that the average kinetic energy remains finite, and the physically relevant competition between kinetic and interaction energy is preserved. For a hypercubic lattice with nearest-neighbor hopping, one chooses

$$
v=\frac{v_{*}}{\sqrt{2 d}},
$$

where $v_{*}$ is kept constant as $d \rightarrow \infty$. While the limit $d \rightarrow \infty$ leads to many simplifications in fermionic lattice models (Vollhardt, 1991, 1993), its most important consequences are arguably the local nature of perturbation theory (Metzner and Vollhardt, 1989) and, in particular, the locality of the selfenergy [Eq. (62)] (Müller-Hartmann, 1989a, 1989b). These properties lead to the mean-field equations (62)-(66), which provide the exact solution of the Hubbard model in the limit of infinite dimensions (Georges and Kotliar, 1992; Jarrell, 1992). A proof can be given in various ways, including a linked cluster expansion around the atomic limit (Metzner, 1991; Georges et al., 1996), a field-theoretical approach (Janiš, 1991; Janiš and Vollhardt, 1992), or the cavity method (Georges et al., 1996).

In the following, we briefly review a diagrammatic argument which shows that for $d \rightarrow \infty$ DMFT reproduces the Feynman diagrams for $\Sigma$ to all orders in perturbation theory. For this purpose one considers the self-energy $\Sigma[G]$ as a functional of the interacting Green's function $G$. In terms of Feynman diagrams, $\Sigma[G]$ is the sum of all self-energy diagrams where internal lines have no self-energy insertions (skeleton diagrams), but represent $G$ instead of $G_{0}$. A power counting argument then shows that for $d \rightarrow \infty$ contributions from nonlocal diagrams should vanish for $\Sigma$ : Each pair of vertices in a skeleton diagram for $\Sigma$ (with space indices $j$ and $l$ ) is at least connected by three independent paths of Green's function lines. If one vertex is an internal vertex, summation over its space index contributes $\sim d^{|l-j|}$ terms with distance $|l-j|$ between $l$ and $j$, while the factor due to the three $G$ lines scales as $\sim d^{-3|l-j| / 2}$ for $d \rightarrow \infty$ due to Eq. (70). Hence only the term $l=j$ survives for $d \rightarrow \infty$. This shows that the functional relation between $G$ and $\Sigma$ is the same as the one for a general single-impurity Anderson model (63),

$$
\Sigma_{i i}[G]=\Sigma_{\text {SIAM }}\left[G_{i i}\right]
$$

By choosing the auxiliary quantity $\Delta_{i}$ such that Eq. (64) yields a given $G_{i i}$, one thus ensures that the self-energy obtained from Eq. (65) gives the correct value of the functional $\Sigma_{i i}[G]$. In this form, the argument was first stated for the FalicovKimball model (Brandt and Mielsch, 1989, 1990, 1991). Because the skeleton expansion is the derivative of the Luttinger-Ward functional (Luttinger and Ward, 1960), $\Sigma[G]=\delta \Phi[G] / \delta G$, DMFT can be rephrased by stating that $\Phi[G]=\sum_{i} \Phi_{\text {SIAM }}\left[G_{i i}\right]$. This statement provides a suitable starting point for the formulation of nonequilibrium DMFT.

\section{Nonequilibrium DMFT formalism}

Quite generally, the mean-field concept can also be used to describe the time evolution of lattice systems, e.g., based on time-dependent Hartree or Gross-Pitaevskii equations. In a direct extension of the static Weiss mean-field theory for spins, the effective Hamiltonian would simply determine the time evolution of $\boldsymbol{S}$, and not only its statistical average. This would result in a nonlinear initial-value problem

$$
\partial_{t}\left\langle\boldsymbol{S}_{i}(t)\right\rangle=\left[\boldsymbol{h}_{i}+\sum_{j} J_{i j}\left\langle\boldsymbol{S}_{j}(t)\right\rangle\right] \times\left\langle\boldsymbol{S}_{i}(t)\right\rangle,
$$

where the term in brackets is the time-dependent mean-field. The initial value is obtained from the static mean-field theory. Similar to the equilibrium case discussed at the beginning of Sec. II.B.1, the time-dependent generalization of static (Hartree) mean-field theory would fail to correctly describe the dynamics of correlated electrons. Instead, one must make the dynamical mean field $\Delta(\omega)$ time dependent, thus going from a Weiss field $\Delta(\omega)$ which captures particle fluctuations in equilibrium to a time-dependent Weiss field $\Delta\left(t, t^{\prime}\right)$ which depends on two times. This defines an effective model for the two-time Green's function $G\left(t, t^{\prime}\right)$ in terms of an effective action that involves only local degrees of freedom coupled to the Weiss field $\Delta\left(t, t^{\prime}\right)$, which in turn depends on $G$ at earlier times.

The precise set of equations can again be obtained from the limit $d \rightarrow \infty$ in the Hubbard model. As long as the total length of the contour is finite, the power counting arguments based on the rescaling (70) remain valid for contour-ordered Green's functions (Schmidt and Monien, 2002). Hence one may conclude that for $d \rightarrow \infty$ the (contour-ordered) self-energy is local in space 


$$
\Sigma_{i j}\left(t, t^{\prime}\right)=\delta_{i j} \Sigma_{i}\left(t, t^{\prime}\right)
$$

(spin indices are suppressed to simplify the notation), such that contour-ordered lattice Green's functions $G_{i j}\left(t, t^{\prime}\right)=$ $-i\left\langle\mathcal{T}_{c} c_{i \sigma}(t) c_{j \sigma}^{\dagger}\left(t^{\prime}\right)\right\rangle$ can be obtained from a Dyson equation (Sec. II.A.1.d)

$$
\left(G^{-1}\right)_{i j}\left(t, t^{\prime}\right)=\left[\delta_{i j}\left(i \partial_{t}+\mu\right)-v_{i j}(t)\right] \delta_{C}\left(t, t^{\prime}\right)-\delta_{i j} \Sigma_{i i}\left(t, t^{\prime}\right),
$$

where the first part $\left(G_{0}^{-1}\right)_{i j}\left(t, t^{\prime}\right)=\left[\delta_{i j}\left(i \partial_{t}+\mu\right)-v_{i j}(t)\right] \delta_{\mathcal{C}}\left(t, t^{\prime}\right)$ is the inverse of the noninteracting lattice Green's function. Furthermore, Eq. (71) still holds, i.e., in order to evaluate the correct functional $\Sigma_{i i}[G]$ in $d \rightarrow \infty$ it is sufficient to solve a general local model with action

$$
S_{i}=-i \int_{C} d t H_{\mathrm{loc}}(t)-i \sum_{\sigma} \int_{\mathcal{C}} d t d t^{\prime} c_{\sigma}^{\dagger}(t) \Delta_{i}\left(t, t^{\prime}\right) c_{\sigma}\left(t^{\prime}\right),
$$

where the auxiliary field $\Delta\left(t, t^{\prime}\right)$ is chosen such that

$$
G_{i i}\left(t, t^{\prime}\right)=-i\left\langle\mathcal{T}_{c} c(t) c^{\dagger}\left(t^{\prime}\right)\right\rangle_{s_{i}},
$$

and $\Sigma$ is implicitly defined via the Dyson equation

$$
G_{i i}^{-1}\left(t, t^{\prime}\right)=\left(i \partial_{t}+\mu\right) \delta_{\mathcal{C}}\left(t, t^{\prime}\right)-\Sigma_{i i}\left(t, t^{\prime}\right)-\Delta_{i}\left(t, t^{\prime}\right) .
$$

Equations (73)-(77) provide the closed set of equations for nonequilibrium DMFT. Although these equations look formally identical to Eqs. (62)-(66) for equilibrium DMFT, they are conceptually very different: As discussed in Sections II.A.1.d and II.A.1.e, Eq. (77) can be viewed as a non-Markovian equation of motion for $G_{i i}$, and through Eq. (71) the memory kernel $\Sigma$ has a nonlinear dependence on $G_{i i}$. Like Eq. (72), nonequilibrium DMFT (on the L-shaped contour $\mathcal{C}$ ) is in essence a nonlinear initial-value problem for $G_{i i}\left(t, t^{\prime}\right)$.

We conclude this section with a brief remark on the limit of $d \rightarrow \infty$ for nonequilibrium. In general, small terms in the Hamiltonian or action can completely modify the long-time behavior of a system, even when they are irrelevant for the equilibrium properties. For example, one may imagine an ideal Fermi gas which is initially excited, such that its momentum distribution $n(\boldsymbol{k}, t=0)$ deviates from a Fermi distribution. For a noninteracting gas, $n(\boldsymbol{k}, t)$ is given by $n(\boldsymbol{k}, 0)$ for all times, but for an arbitrary weak interaction $n(\boldsymbol{k}, t)$ is supposed to eventually reach a thermal equilibrium distribution. Hence the limit $t \rightarrow \infty$ is necessarily nonperturbative in terms of the interaction. In the same way, one cannot generally expect the limit of $t \rightarrow \infty$ and $d \rightarrow \infty$ to commute. In the Weiss mean-field theory [which is exact for $d=\infty$ with the scaling $J \sim J_{*} / d$ (Brout, 1960)], e.g., spin-flip terms are absent in a collinear antiferromagnet, but in a real system such terms may completely modify the long-time limit. The importance of nonlocal effects on the long-time dynamics of strongly correlated systems is an open question that can finally only be addressed by extensions of DMFT (some of which are mentioned in Sec. II.E.2).

\section{Models}

a. Overview

Nonequilibrium DMFT can be applied to a large class of problems, including arbitrary electromagnetic driving fields, dissipative and nondissipative systems, and many different types of local interaction terms. The general lattice Hamiltonian for the relevant (nondissipative) models is of the form

$$
H=\sum_{i j, \alpha \alpha^{\prime}} v_{i \alpha j \alpha^{\prime}}(t) c_{i \alpha}^{\dagger} c_{j \alpha^{\prime}}+\sum_{i} H_{\mathrm{loc}}^{(i)}
$$

where $i$ and $\alpha$ denote site and orbital or spin labels. The second term in this equation is a sum of local interaction and singleparticle terms. Apart from the Hubbard model [Eq. (61)], important examples include (i) the periodic Anderson model

$$
H_{\mathrm{loc}}=U f_{\uparrow}^{\dagger} f_{\uparrow} f_{\downarrow}^{\dagger} f_{\downarrow}+E_{f} \sum_{\sigma} f_{\sigma}^{\dagger} f_{\sigma}+\sum_{\sigma}\left(V f_{\sigma}^{\dagger} c_{\sigma}+\text { H.c. }\right),
$$

where conduction electrons hybridize with localized $f$ orbitals; (ii) the Kondo lattice model

$$
H_{\mathrm{loc}}=J \sum_{\sigma, \sigma^{\prime}} c_{\sigma}^{\dagger} \boldsymbol{\tau}_{\sigma \sigma^{\prime}} c_{\sigma^{\prime}} \cdot \boldsymbol{S},
$$

which describes electrons (with spin $\tau$ ) that interact with a local spin $S$; (iii), the Holstein model

$$
H_{\mathrm{loc}}=\omega_{0}\left(b^{\dagger} b+\frac{1}{2}\right)+g\left(b^{\dagger}+b\right)\left(n_{\uparrow}+n_{\downarrow}-1\right),
$$

for electron-phonon coupled systems; and (iv) the HubbardHolstein model

$$
H_{\mathrm{loc}}=U n_{\uparrow} n_{\downarrow}+\omega_{0}\left(b^{\dagger} b+\frac{1}{2}\right)+g\left(b^{\dagger}+b\right)\left(n_{\uparrow}+n_{\downarrow}-1\right) .
$$

The first two models play an important role in the theory of heavy fermion materials (Coleman, 2007), and all four have been studied intensively within equilibrium DMFT (Meyer, Hewson, and Bulla, 2002; Werner and Millis, 2007; De Leo, Civelli, and Kotliar, 2008; Otsuki, Kusunose, and Kuramoto, 2009).

The Falicov-Kimball model

$$
H=\sum_{i j} v_{i j}(t) c_{i}^{\dagger} c_{j}+E_{f} \sum_{i} f_{i}^{\dagger} f_{i}+U \sum_{i} f_{i}^{\dagger} f_{i} c_{i}^{\dagger} c_{i}
$$

describes itinerant $c$ electrons and immobile $f$ electrons on a lattice, interacting via a repulsive local interaction $U$ (Falicov and Kimball, 1969; Brandt and Mielsch, 1989; Freericks and Zlatić, 2003).

\section{b. Time-dependent electric fields}

The first term in Eq. (78), with arbitrary hoppings $v_{i \alpha j \alpha^{\prime}}(t)$, can include time-dependent electromagnetic fields (Sec. III.A). For a single-band model, the Peierls substitution (Peierls, 1933; Luttinger, 1951; Kohn, 1959) introduces the 
vector potential $\boldsymbol{A}(\boldsymbol{r}, t)$ as a phase factor in the hopping matrix elements

$$
v_{i j}(t)=v_{i j} \exp \left(-\frac{i e}{\hbar} \int_{\boldsymbol{R}_{i}}^{\boldsymbol{R}_{j}} d \boldsymbol{r} \cdot \boldsymbol{A}(\boldsymbol{r}, t)\right)
$$

and adds a scalar potential term $e \sum_{i \sigma} \Phi\left(\boldsymbol{R}_{i}, t\right) c_{i \sigma}^{\dagger} c_{i \sigma}$ to the Hamiltonian ( $e$ is the charge of an electron). (We use units in which the speed of light is set to $c=1$.) The Peierls substitution can apparently not describe interband Zener tunneling in multiband systems, because dipole matrix elements between bands of different symmetry are neglected (Foreman, 2002). We will not discuss interband tunneling in this review. On a technical level, however, the DMFT formalism will not be modified when those dipole matrix elements are incorporated into the single-particle part of the general model [Eq. (78)].

The Peierls substitution is derived from the requirement that the Hamiltonian is invariant under the gauge transformation

$$
\begin{gathered}
c_{j \sigma} \rightarrow c_{j \sigma} \exp \left(\frac{i e}{\hbar} \chi\left(\boldsymbol{R}_{j}, t\right)\right), \\
\boldsymbol{A}(\boldsymbol{r}, t) \rightarrow \boldsymbol{A}(\boldsymbol{r}, t)+\nabla \chi(\boldsymbol{r}, t), \\
\Phi(\boldsymbol{r}, t) \rightarrow \Phi(\boldsymbol{r}, t)-\frac{\partial \chi(\boldsymbol{r}, t)}{\partial t} .
\end{gathered}
$$

The (gauge-invariant) current operator can be obtained from the derivative $\boldsymbol{j}(\boldsymbol{r})=-\delta H / \delta A(\boldsymbol{r})$ (Scalapino, White, and Zhang, 1992), such that it satisfies the continuity equation for the charge density $\rho(\boldsymbol{r})=e \sum_{i \sigma} \delta\left(\boldsymbol{r}-\boldsymbol{R}_{i}\right) c_{i \sigma}^{\dagger} c_{i \sigma}$. Usually one is concerned with situations in which the applied field varies only slowly on the atomic scale, which is the case even for optical frequencies. When the $\boldsymbol{r}$ dependence of $\boldsymbol{A}$ is neglected, the Peierls substitution leads to a time-dependent dispersion

$$
\epsilon_{k}(t)=\epsilon\left(\boldsymbol{k}-\frac{e}{\hbar} \boldsymbol{A}(t)\right)
$$

where $\epsilon(\boldsymbol{k})$ is the dispersion for zero field, and $a$ is the lattice spacing, so that the hopping part of the Hamiltonian reads $H=\sum_{k \sigma} \epsilon_{\boldsymbol{k}}(t) c_{\boldsymbol{k} \sigma}^{\dagger} c_{\boldsymbol{k} \sigma}$. Correspondingly, the current operator in the limit of long (optical) wavelengths becomes

$$
j(t)=\frac{e}{V} \sum_{k \sigma} v_{k}(t) n_{k \sigma},
$$

where $V$ is the volume, and $v_{\boldsymbol{k}}$ is the group velocity of the Bloch electrons

$$
\boldsymbol{v}_{\boldsymbol{k}}(t)=\frac{1}{\hbar} \partial_{\boldsymbol{k}} \epsilon_{\boldsymbol{k}}(t)=\frac{1}{\hbar} \partial_{\boldsymbol{k}} \epsilon\left(\boldsymbol{k}-\frac{e}{\hbar} \boldsymbol{A}(t)\right) .
$$

Note that although the right-hand side of Eq. (87) involves gauge dependent quantities, the current itself is of course gauge independent (see also the later discussion in Sec. II.B.5.a).

\section{c. Dissipative systems}

To describe dissipation of energy to other degrees of freedom, the Hamiltonian (78) may be coupled to some environment, like in Eq. (51). When the environment is traced out, one obtains an effective description of the system with an additional self-energy contribution $\Sigma_{\text {bath }}$ :

$$
\Sigma=\Sigma_{\text {bath }}+\Sigma_{\text {loc }}
$$

Here $\Sigma_{\text {loc }}$ contains all diagrams due the local interaction $H_{\text {loc }}$. In the spirit of DMFT, it is local and will be evaluated by the solution of the impurity model, i.e., the functional relation (71) is not modified by the dissipation. For a general Hamiltonian (51), this decoupling into bath and interaction self-energies is not exact, as it neglects vertex corrections. However, when discussing dissipation one is interested in a regime in which results become universal with respect to the type of dissipation. This is usually the regime of weak systembath coupling. The decoupling (89) fails in the opposite limit of strong system-bath coupling, where the dynamics is clearly no longer universal and the bath should rather be considered as an integral part of the system.

Two dissipation mechanisms have so far been considered within DMFT: (i) the free-fermion bath (Büttiker model, Sec. II.A.2.b), in which one additional reservoir of particles is coupled to each lattice site (Tsuji, Oka, and Aoki, 2009; Amaricci et al., 2012; Aron, 2012; Werner and Eckstein, 2012; Han, 2013), and (ii) the coupling to a bath of harmonic oscillator modes (Eckstein and Werner, 2013a, 2013c; Kemper et al., 2013). For the Büttiker model, the systembath coupling is bilinear in the fermion creation and annihilation operators, such that additional degrees of freedom can be integrated out exactly. Equation (89) is then exact with $\Sigma_{\text {bath }}$ given by Eq. (55). In spite of its apparent simplicity and the absence of momentum scattering, the model can lead to a physically meaningful steady state (Amaricci et al., 2012; Han, 2013). For the bosonic bath, one considers an infinite set of phonons, coupled via the Holstein coupling (81). In this case the decoupling in bath and interaction self-energies holds only at weak coupling. Consequently, we take Eq. (89) with a first-order electron-phonon diagram

$$
\begin{gathered}
\Sigma_{\mathrm{bath}}[G]=g^{2} G\left(t, t^{\prime}\right) D\left(t, t^{\prime}\right), \\
D\left(t, t^{\prime}\right)=-i \int d \omega e^{-i \omega\left(t-t^{\prime}\right)} \Gamma(\omega)\left[\theta_{C}\left(t, t^{\prime}\right)+b(\omega)\right],
\end{gathered}
$$

where $D$ is the propagator for free bosons with a density of states $\Gamma(\omega), b(\omega)=1 /\left(e^{\beta \omega}-1\right)$ is the bosonic occupation function, and $g$ measures the coupling strength. In general, the precise mechanism of dissipation should be irrelevant for the physics. The fermion bath has the conceptual advantage of treating the system-bath coupling exactly, while the phonon bath is particle-number conserving by construction.

In pump-probe spectroscopy experiments on solids, the lattice acts as a heat bath for the electrons, but in many situations a quasiequilibrium description such as the twotemperature model (Allen, 1987) is not applicable. It is therefore important to develop a formalism which can treat the quantum-mechanical time evolution of electron-phonon 
coupled systems, such as Eqs. (81) and (82). Lattice perturbation theory has been used by Sentef et al. (2012) to study the time evolution of a photoexcited electron-phonon system in the weak correlation regime. A DMFT formalism for the Holstein-Hubbard model, which captures the effect of the nonequilibrium state of the electrons on the evolution of the phonons, and the feedback of the phonons on the electronic relaxation, has recently been presented by Werner and Eckstein (2013).

\section{Implementation of the self-consistency}

a. Stable time propagation scheme

Typically, the self-consistent solution of the DMFT equations is achieved by some kind of iterative procedure (Fig. 1): Starting from some guess for $\Delta$, one must (i) compute $G$ [Eq. (76)], (ii) solve Eq. (77) for $\Sigma$, (iii) solve Eq. (74) for $G_{i i}$, (iv) solve Eq. (77) with the new $G$ to get a new $\Delta$, and iterate steps (i)-(iv) until convergence is reached. In nonequilibrium DMFT, this procedure can be implemented as a timepropagation scheme: If self-consistent solutions $G\left(t_{1}, t_{2}\right)$ and $\Delta\left(t_{1}, t_{2}\right)$ have been obtained for $t_{1}, t_{2} \leq t$, one can extrapolate $\Delta$ to the next time step $\left(t_{1}=t+\Delta t\right.$ or $t_{2}=t+\Delta t$ ) and again converge steps (i)-(iv), thereby updating only the data at $t_{1}=t+\Delta t$ or $t_{2}=t+\Delta t$. Matsubara functions are obtained from a separate equilibrium DMFT calculation, i.e., by iterating steps (i)-(iv) for Green's functions on the imaginary branch of $\mathcal{C}$.

In this section we discuss the self-consistency part in the above procedure [steps (ii)-(iv)], while the impurity solver [step (i)] is deferred to Sec. II.C. In contrast to equilibrium DMFT, the self-consistency can be numerically costly, as one must manipulate contour-ordered Green's functions that depend on two time variables. For an arbitrary spatially inhomogeneous system with $L$ inequivalent lattice sites, the memory to store a Green's function $G_{i j}$ scales like $N^{2} L^{2}$ (where $N$ is the number of time-discretization steps), which can reach a terabyte for $N \approx 1000$ and $L \approx 100$. In this section we restrict the discussion to translationally invariant systems. The inhomogeneous problem can be handled for layered systems, as discussed in Sec. II.E.4.

The problem of computing $\Sigma$ [step (ii) above] or $\Delta$ [step (iv)] from Eq. (77) is not a contour equation of the type (40) or (44), whose numerical solution was discussed in Sec. II.A.1.e. In contrast, it seems more closely related to the numerically less favorable Volterra equations of the first kind (45). One can solve these equations in time-discretized form on the full contour (Freericks, Turkowski, and Zlatić, 2006; Freericks, 2008), but with a slight reformulation it is also possible to take full advantage of the Kadanoff-Baym propagation scheme discussed in Sec. II.A.1.e. For this purpose we introduce the isolated impurity Green's function $g$, which is defined via the impurity Dyson equation for $\Delta=0$ :

$$
g^{-1}\left(t, t^{\prime}\right)=\left(i \partial_{t}+\mu\right) \delta_{\mathcal{C}}\left(t, t^{\prime}\right)-\Sigma\left(t, t^{\prime}\right)
$$

(For simplicity, the following equations are first stated for the single-band case without spin dependence.) In order to compute $g$ one can reformulate Eq. (77) in an integral form

$$
\begin{aligned}
G & =g+g * \Delta * G \\
& =g+G * \Delta * g,
\end{aligned}
$$

which leads to a Kadanoff-Baym equation of the type (44):

$$
[1+F] * g=Q, \quad F=G * \Delta, \quad Q=G .
$$

[If the impurity solver gives $\Sigma$, as is the case for the weakcoupling solver (Sec. II.C.4) and continuous-time quantum Monte Carlo (Sec. II.C.3), solving for $g$ is a standard Kadanoff-Baym equation (40).]

Next one computes momentum-resolved Green's functions $G_{k}$ from the Dyson equation (74) in the momentum representation

$$
G_{\boldsymbol{k}}^{-1}=\left(i \partial_{t}+\mu\right) \delta_{C}\left(t, t^{\prime}\right)-\Sigma\left(t, t^{\prime}\right)-\epsilon_{\boldsymbol{k}}\left(t, t^{\prime}\right) \equiv g^{-1}-\epsilon_{\boldsymbol{k}},
$$

with $\epsilon_{k}\left(t, t^{\prime}\right)=\delta_{C}\left(t, t^{\prime}\right) \epsilon_{k}(t)$. It reads in the integral form,

$$
G_{k}=g+g * \epsilon_{k} * G_{k} .
$$

Hence, $G_{\boldsymbol{k}}$ can again be obtained by solving Kadanoff-Baym equations of the type (44):

$$
\left[1+F_{k}\right] * G_{k}=Q_{k}, \quad F_{k}=-g * \epsilon_{k}, \quad Q_{k}=g .
$$

To compute the updated $\Delta$ we start by summing Eq. (95) over $\boldsymbol{k}$ :

$$
G=g+g * \sum_{k}\left(\epsilon_{k} * G_{k}\right),
$$

where we used the normalization $\sum_{k}=1$, and $\sum_{k} G_{k}=G$. Comparison with Eq. (92a) gives

$$
\Delta * G=\sum_{k} \epsilon_{k} * G_{k} \equiv G_{1}
$$

Solving this integral equation for $\Delta$ would still be an integral equation of the less stable type (45). However, after inserting the conjugate of Eq. (95) and Eq. (92b) into the right-hand side and left-hand side of Eq. (98a), respectively, one finds

$$
\Delta+\Delta * G * \Delta=\sum_{k}\left(\epsilon_{k}+\epsilon_{k} * G_{k} * \epsilon_{k}\right) \equiv G_{2},
$$

such that also $\Delta$ can be obtained from a Kadanoff-Baym equation (44):

$$
\left[1+G_{1}\right] * \Delta=G_{2} .
$$

In an implementation of nonequilibrium DMFT that uses this scheme, steps (ii) and (iii) above are replaced by the solution of Eqs. (93) and (96), respectively. Instead of step (iv), one computes the $\boldsymbol{k}$ sums (98a) and (98b) and solves Eq. (98c) for a new $\Delta$ (Eckstein and Werner, 2011b).

If one adopts an impurity solver based on the weakcoupling expansion (Sec. II.C.4), one can skip step (ii), as $\Sigma$ is directly given by a functional of the Weiss Green's function $\mathcal{G}_{0}$, which is defined by 


$$
\mathcal{G}_{0}^{-1}\left(t, t^{\prime}\right)=\left(i \partial_{t}+\mu\right) \delta_{\mathcal{C}}\left(t, t^{\prime}\right)-\Delta\left(t, t^{\prime}\right)
$$

In this case, one can take $\mathcal{G}_{0}$ to represent the dynamical mean field, and $\Delta$ does not explicitly appear in the self-consistency calculation. In step (iii), one solves the Kadanoff-Baym equation (74) for $G$ with the given $\Sigma$. A new $\mathcal{G}_{0}$ is derived from the impurity Dyson equation of the form

$$
[1+F] * \mathcal{G}_{0}=Q, \quad F=G * \Sigma, \quad Q=G
$$

For dissipative systems, it is convenient to include the dissipative self-energy (89) into the definition of $g$ :

$$
g^{-1}\left(t, t^{\prime}\right)=\left(i \partial_{t}+\mu\right) \delta_{\mathcal{C}}\left(t, t^{\prime}\right)-\Sigma\left(t, t^{\prime}\right)-\Sigma_{\text {bath }}\left(t, t^{\prime}\right)
$$

and define a corresponding lattice hybridization function $\Delta_{\text {lat }}=\Delta-\Sigma_{\text {bath }}$. Then Eqs. (92)-(98) hold with the replacement $\Delta \rightarrow \Delta_{\text {lat }}$, and the only additional step in the DMFT selfconsistency is to compute the hybridization function $\Delta$ from $\Delta=\Delta_{\text {lat }}+\Sigma_{\text {bath }}$ (Eckstein and Werner, 2013c).

\section{b. Momentum summations}

The momentum summations appearing in the DMFT selfconsistency [e.g., Eqs. (98a) and (98b)] can be simplified in special situations. First, without external electromagnetic fields $\epsilon_{\boldsymbol{k}}$ is time independent, such that $\boldsymbol{k}$-dependent quantities depend on $\boldsymbol{k}$ only via $\epsilon_{\boldsymbol{k}}$, and momentum sums can be reduced to integrals over a one-dimensional density of states

$$
\sum_{\boldsymbol{k}} g\left(\epsilon_{\boldsymbol{k}}\right)=\int d \epsilon g(\epsilon) \sum_{\boldsymbol{k}} \delta\left(\epsilon-\epsilon_{\boldsymbol{k}}\right) \equiv \int d \epsilon D(\epsilon) g(\epsilon) .
$$

In particular, for a semielliptic density of states (69) one can collapse the whole self-consistency into a single equation $\Delta\left(t, t^{\prime}\right)=v_{*}^{2} G\left(t, t^{\prime}\right)$ (Eckstein, Hackl et al., 2009), like for equilibrium [Eq. (67)]. If the hopping is such that the (positive) bandwidth $\left(=4 v_{*}\right)$ depends on time, this equation generalizes to (Eckstein and Kollar, 2010)

$$
\Delta\left(t, t^{\prime}\right)=v_{*}(t) G\left(t, t^{\prime}\right) v_{*}\left(t^{\prime}\right) .
$$

The situation is more involved in the presence of electromagnetic fields, where one might have to do the $\boldsymbol{k}$ sum explicitly. A simplification is possible for a hypercubic lattice with bare dispersion $\epsilon_{k}=-\left(2 v_{*} / \sqrt{2 d}\right) \sum_{\alpha} \cos \left(k_{\alpha}\right)$ and Gaussian density of states

$$
D(\epsilon)=\frac{1}{\sqrt{2 \pi} v_{*}} e^{-\epsilon^{2} / 2 v_{*}^{2}},
$$

when a homogeneous field $\boldsymbol{A}=A(t)(1,1,1, \ldots)$ points along the body diagonal of the unit cell (Turkowski and Freericks, 2005). One has

$$
\begin{aligned}
\epsilon(\boldsymbol{k}-\boldsymbol{A}(t)) & =-2 v \sum_{\alpha} \cos \left(k_{\alpha}-A(t)\right) \\
& =\cos (A(t)) \epsilon_{\boldsymbol{k}}+\sin (A(t)) \bar{\epsilon}_{\boldsymbol{k}},
\end{aligned}
$$

with $\quad \bar{\epsilon}_{k}=-2 v \sum_{\alpha} \sin \left(k_{\alpha}\right) \quad$ (taking $\quad a=e=\hbar=1$ ). Momentum summations then reduce to integrals over a two-dimensional joint density of states $D(\epsilon, \bar{\epsilon})=$ $\sum_{k} \delta\left(\epsilon-\epsilon_{k}\right) \delta\left(\bar{\epsilon}-\bar{\epsilon}_{k}\right)$ :

$$
\sum_{\boldsymbol{k}} g(\epsilon(\boldsymbol{k}-\boldsymbol{A}))=\int \operatorname{d\epsilon } \bar{\epsilon} D(\epsilon, \bar{\epsilon}) g(\epsilon \cos (A)+\bar{\epsilon} \sin (A)) .
$$

For the hypercubic lattice, one has $D(\epsilon, \bar{\epsilon})=D(\epsilon) D(\bar{\epsilon})$ (Turkowski and Freericks, 2005). For other examples of an infinite-dimensional lattice structure, see Tsuji, Oka, and Aoki (2008).

\section{Observables and conservation laws}

\section{a. Equal-time observables}

Equal-time observables [Eq. (6)] of the lattice model can directly be computed from the lattice Green's functions. Before we define the observables, we remark that in the presence of external electromagnetic fields the Green's functions are not a priori gauge invariant. Under the gauge transformation (85), the Green's function $G_{i j}\left(t, t^{\prime}\right)=$ $-i\left\langle\mathcal{T}_{c} c_{i}(t) c_{j}^{\dagger}\left(t^{\prime}\right)\right\rangle$ transforms as

$$
G_{i j}\left(t, t^{\prime}\right) \rightarrow G_{i j}\left(t, t^{\prime}\right) \exp \left(\frac{i e}{\hbar}\left[\chi\left(\boldsymbol{R}_{i}, t\right)-\chi\left(\boldsymbol{R}_{j}, t^{\prime}\right)\right]\right) .
$$

Since physical observables should not depend on the choice of the gauge, the gauge dependent Green's function cannot be generally used in the present form. A widely adopted prescription is to put an additional phase factor ("string") to the Green's function (Boulware, 1966; Davies and Wilkins, 1988; Bertoncini and Jauho, 1991)

$\tilde{G}_{i j}\left(t, t^{\prime}\right)=\exp \left(\frac{-i e}{\hbar} \int_{\left(\boldsymbol{R}_{j}, t^{\prime}\right)}^{\left(\boldsymbol{R}_{i}, t\right)}[d \overline{\boldsymbol{r}} \cdot \boldsymbol{A}(\overline{\boldsymbol{r}}, \bar{t})-d \bar{t} \Phi(\overline{\boldsymbol{r}}, \bar{t})]\right) G_{i j}\left(t, t^{\prime}\right)$.

The phase factor cancels the change of the phase of $G$ (107), hence $\tilde{G}$ remains gauge invariant. However, $\tilde{G}$ depends on the path of the line integral in the exponential. A standard convention is to take a straight line in the four-dimensional spacetime connecting $\left(\boldsymbol{R}_{i}, t\right)$ and $\left(\boldsymbol{R}_{j}, t^{\prime}\right)$ (Boulware, 1966).

For a uniform electric field which is often studied with the nonequilibrium DMFT (see Sec. III.A), one can take the temporal gauge [ $\Phi=0$ and $\boldsymbol{A}=\boldsymbol{A}(t)]$. In this case (which is focused on in the following), the local Green's function is gauge invariant $G_{i i}\left(t, t^{\prime}\right)=\widetilde{G}_{i i}\left(t, t^{\prime}\right)$. The equal-time Green's function, on the other hand, becomes gauge invariant if one shifts the momentum $G_{k+A(t)}(t, t)=\tilde{G}_{k}(t, t)$.

Using the gauge invariant Green's function, one can safely construct physical observables. For example, the number of particles on site $i$ with spin $\sigma$ is

$$
n_{i, \sigma}(t)=\left\langle c_{i \sigma}^{\dagger}(t) c_{i \sigma}(t)\right\rangle=-i G_{i i, \sigma}^{<}(t, t)=-i \tilde{G}_{i i, \sigma}^{<}(t, t),
$$

and the current (87) is 


$$
\begin{aligned}
j(t) & =-\frac{i e}{V} \sum_{k \sigma} v_{k-A(t)} G_{k, \sigma}^{<}(t, t)=-\frac{i e}{V} \sum_{k \sigma} v_{k} G_{k+A(t), \sigma}^{<}(t, t) \\
& =-\frac{i e}{V} \sum_{k \sigma} v_{k} \tilde{G}_{k, \sigma}^{<}(t, t),
\end{aligned}
$$

both of which are explicitly gauge invariant. The momentum occupation defined by $n(\boldsymbol{k}, t)=-i G_{\boldsymbol{k}, \sigma}^{<}(t, t)$ is apparently not gauge invariant. Instead, one can take a comoving wave vector $\boldsymbol{k}=\boldsymbol{k}+\boldsymbol{A}(t)$ (Davies and Wilkins, 1988), where

$$
n(\tilde{\boldsymbol{k}}, t)=-i G_{\boldsymbol{k}+\boldsymbol{A}(t), \sigma}^{<}(t, t)=-i \tilde{G}_{\boldsymbol{k}, \sigma}^{<}(t, t)
$$

becomes gauge invariant, and hence can be interpreted as a physically meaningful observable.

Energies are also calculated from the Green's functions. The kinetic energy per spin and site is (denoting the number of sites by $L$ )

$$
E_{\mathrm{kin}}(t)=\frac{-i}{L} \sum_{j l} v_{j l}(t) G_{l j, \sigma}^{<}(t, t)=\frac{-i}{L} \sum_{j}\left[\Delta_{j} * G_{j j, \sigma}\right]^{<}(t, t),
$$

where the second equation follows from a comparison of Eqs. (74) and (77). The interaction energy can be computed from the self-energy, using equations of motion. In general, comparison of the equation of motion and the lattice Dyson equation gives

$$
\left\langle c_{i \alpha}^{\dagger}\left[H_{\mathrm{loc}}, c_{i \alpha^{\prime}}\right]\right\rangle=i \sum_{\alpha^{\prime \prime}}\left[\Sigma_{i \alpha, i \alpha^{\prime \prime}} * G_{i \alpha^{\prime \prime}, i \alpha^{\prime}}\right]^{<}(t, t) .
$$

For the Hubbard interaction (61) and a homogeneous state, one has

$$
U(t)\left\langle n_{i \sigma}(t)\left[n_{i \bar{\sigma}}(t)-\frac{1}{2}\right]\right\rangle=-i\left[\sum * G_{i i, \sigma}\right]^{<}(t, t),
$$

which allows one to compute the double occupancy $d(t)=\left\langle n_{i \uparrow}(t) n_{i \downarrow}(t)\right\rangle$.

Finally we remark that DMFT (with an exact impurity solver) is a conserving approximation in the sense of Baym and Kadanoff (Baym and Kadanoff, 1961; Baym, 1962), because the self-energy is related to the Green's function as a functional derivative of a Luttinger-Ward functional. The latter can be used to prove, along the lines of Baym (1962), particlenumber conservation and energy conservation

$$
\frac{d}{d t}\langle H(t)\rangle=\boldsymbol{j}(t) \cdot \boldsymbol{E}(t),
$$

where $\boldsymbol{E}$ is the electric field.

\section{b. Photoemission spectrum}

Time- and angular-resolved photoemission spectroscopy (ARPES) is the most direct experimental tool to probe the time evolution of both the electronic spectrum and the occupation on ultrafast time scales. Static photoemission spectroscopy in equilibrium is often analyzed in terms of the momentumresolved spectral function

$$
I\left(\boldsymbol{k}_{f}, E\right)=\sum_{\boldsymbol{k}}\left|M_{\boldsymbol{k}}\right|^{2} \delta_{\boldsymbol{k}_{\|}+\boldsymbol{q}_{\|}, \boldsymbol{k}_{f \|}} N_{\boldsymbol{k}}\left(E-\hbar \omega_{q}-W\right),
$$

where $N_{k}(\omega)=f(\omega) A_{k}(\omega)$ is the occupied density of states at momentum $\boldsymbol{k}$ [cf. Eq. (28a)], $\boldsymbol{q}$ is the momentum of the incoming photon, and $I\left(\boldsymbol{k}_{f}, E\right)$ is the photoemission intensity at final momentum $\boldsymbol{k}_{f}$ and energy $E$, and $W$ is the work function. The delta function accounts for momentum conservation parallel to the surface, and the $M_{k}$ denote matrix elements, which are often taken as $\boldsymbol{k}$ independent as a first approximation. The most important approximation entering Eq. (116) is the so-called sudden approximation (Hedin and Lee, 2002), which neglects interactions between the outgoing electron and the bulk and thus allows one to express the photoelectron current in terms of single-particle properties of the sample.

In time-resolved ARPES one probes the state of a system with a short pulse with center frequency $\Omega$, and counts the total number of electrons emitted with a certain momentum $\boldsymbol{k}_{f}$ and energy $E$. The electric field of the probe pulse is of the form $\cos \left[\Omega\left(t-t_{p}+\phi\right)\right] S\left(t-t_{p}\right)$, where $S(t)$ is the probe envelope, $t_{p}$ is the probing time, and $\phi$ is the carrier-envelope phase. The system can be in an arbitrary nonequilibrium state due to an earlier pump excitation. Equation (116) can directly be generalized to this situation (Freericks, Krishnamurthy, and Pruschke, 2009): In the sudden approximation, the electric probe field couples the electronic orbitals in the sample to the outgoing electron states $\left|\boldsymbol{k}_{f}\right\rangle$ via some dipole matrix element. If we again disregard for a moment the $\boldsymbol{k}$ dependence of this matrix element, straightforward second-order time-dependent perturbation theory gives (after averaging over the carrierenvelope phase $\phi$ )

$$
I\left(\boldsymbol{k}_{f}, E ; t_{p}\right) \propto \sum_{\boldsymbol{k}} \delta_{\boldsymbol{k}_{\|}+\boldsymbol{q}_{\|}, \boldsymbol{k}_{f \|}} I_{\boldsymbol{k}}\left(E-\hbar \omega_{q}-W ; t_{p}\right),
$$

$$
I_{k}\left(\omega ; t_{p}\right)=-i \int d t d t^{\prime} S(t) S\left(t^{\prime}\right) e^{i \omega\left(t^{\prime}-t\right)} \tilde{G}_{k}^{<}\left(t+t_{p}, t^{\prime}+t_{p}\right),
$$

where $\tilde{G}_{k}$ is the Fourier transform of the gauge-invariant Green's function (108). This expression provides a convenient starting point to analyze time-resolved ARPES in terms of the contour Green's functions determined in DMFT. [In contrast, the imaginary part of a partial Fourier transform $G^{<}(\omega, t)=$ $\int d \bar{t} e^{i \omega \bar{t}} G^{<}(t+\bar{t} / 2, t-\bar{t} / 2)$ is not always positive.] The equation contains the fundamental frequency-time uncertainty (Eckstein and Kollar, 2008a): When the probe pulse is very short, $S(t)=\delta(t)$, one measures instantaneous occupations $I_{k}\left(\omega ; t_{p}\right)=n_{k}\left(t_{p}\right)$ but all energy resolution is lost. In the limiting case of a stationary state, Eq. (118) reduces to a convolution of the equilibrium result (116) with the spectral density $|\tilde{S}(\omega)|^{2}$ of the probe pulse. For a quasistationary state, when $G\left(t, t^{\prime}\right)$ can be approximated as translationally invariant in time during the probe pulse, $I_{k}\left(\omega ; t_{p}\right)$ is given by a corresponding convolution of the partial Fourier transform 
$G^{<}\left(\omega, t_{p}\right)$. Applications of this formula are discussed in Sec. III.A.3.

\section{c. Optical conductivity}

Another very powerful tool to probe the time evolution of strongly correlated systems is the electromagnetic response, ranging from terahertz, suitable for the analysis of carrier mobilities or phonons, to optical frequencies, which can be used to study, e.g., interband or charge transfer excitations. In this frequency range, the electromagnetic response is described by the conductivity in the limit $\boldsymbol{q} \rightarrow 0$, i.e., the response of a translationally invariant current $j$ to a translationally invariant electric probe field $\delta \boldsymbol{E}$ :

$$
\sigma_{\alpha \beta}\left(t, t^{\prime}\right)=\frac{\delta\left\langle j_{\alpha}(t)\right\rangle}{\delta E_{\beta}\left(t^{\prime}\right)}
$$

$\left[\delta j_{\alpha}(t)=\int_{-\infty}^{t} d \bar{t} \sigma_{\alpha \beta}(t, \bar{t}) \delta E_{\beta}(\bar{t})\right.$ in integral notation]. This response function of the nonequilibrium state can be computed from the contour-ordered current-current correlation function (Kubo relation), which can be evaluated in nonequilibrium DMFT (Eckstein and Kollar, 2008c; Tsuji, Oka, and Aoki, 2009) in analogy to equilibrium (Pruschke, Cox, and Jarrell, 1993). For this purpose it is convenient to define the susceptibility

$$
\chi_{\alpha \beta}\left(t, t^{\prime}\right)=\frac{\delta\left\langle j_{\alpha}(t)\right\rangle}{\delta A_{\beta}\left(t^{\prime}\right)}
$$

which is related to the optical conductivity $\sigma_{\alpha \beta}\left(t, t^{\prime}\right)$ via

$$
\sigma_{\alpha \beta}\left(t, t^{\prime}\right)=-\int_{t^{\prime}}^{t} d \bar{t} \chi_{\alpha \beta}(t, \bar{t}) \quad\left(t \geq t^{\prime}\right),
$$

within the chosen gauge $\boldsymbol{E}(t)=-\partial_{t} \boldsymbol{A}(t)$. The susceptibility (120) can be obtained by taking the derivative of Eq. (110), where the vector potential enters both in the vertex $v_{k}(t)$ (leading to the diamagnetic contribution to $\chi$ ) and in the Green's function $G_{k \sigma}^{<}(t, t)$ (leading to the paramagnetic contribution) (Eckstein and Kollar, 2008c),

$$
\begin{array}{r}
\chi_{\alpha \beta}\left(t, t^{\prime}\right)=\chi_{\alpha \beta}^{\mathrm{dia}}\left(t, t^{\prime}\right)+\chi_{\alpha \beta}^{\mathrm{pm}}\left(t, t^{\prime}\right), \\
\chi_{\alpha \beta}^{\mathrm{dia}}\left(t, t^{\prime}\right)=-\frac{i e}{V} \sum_{k \sigma} \frac{\delta v_{\boldsymbol{k}}^{\alpha}(t)}{\delta A_{\beta}\left(t^{\prime}\right)} G_{\boldsymbol{k} \sigma}^{<}(t, t), \\
\chi_{\alpha \beta}^{\mathrm{pm}}\left(t, t^{\prime}\right)=-\frac{i e}{V} \sum_{\boldsymbol{k} \sigma} v_{\boldsymbol{k}}^{\alpha}(t) \frac{\delta G_{\boldsymbol{k} \sigma}^{<}(t, t)}{\delta A_{\beta}\left(t^{\prime}\right)} .
\end{array}
$$

The paramagnetic contribution can be found from a variation of the lattice Dyson equation (94):

$$
\delta G_{k \sigma}=-G_{k \sigma} *\left[-\delta \epsilon_{k}-\delta \Sigma_{\sigma}\right] * G_{k \sigma},
$$

where the term in brackets is $\delta G_{k}^{-1}$. In equilibrium DMFT, the contribution from $\delta \Sigma$, which is related to the vertex $\delta \Sigma / \delta G$ of the impurity model, vanishes in Eq. (122c) because (i) $\Sigma$ is $\boldsymbol{k}$ independent, and (ii) $G_{k \sigma} G_{k \sigma}$ and $v_{k \sigma}$ are even and odd with respect to $\boldsymbol{k}$, respectively (Khurana, 1990). This symmetry argument leads to the drastic simplification that the conductivity can be expressed in terms of the product of two Green's functions (bubble diagram), without local vertex corrections. In nonequilibrium, conditions (ii) can be violated in several ways: The vertex (88) is no longer antisymmetric when an electric field is present in addition to the probe field, e.g., when the system is constantly driven by an ac field (Tsuji, Oka, and Aoki, 2009), or when the pump and probe fields overlap in time. Furthermore, the isotropy $G_{k \sigma}=G_{-k \sigma}$ is lost whenever the system is driven out of equilibrium by a polarized pump pulse (the system is clearly not isotropic as long as a current is flowing). However, in this case the isotropy of $G_{k}$ is often restored on a much faster time scale than other interesting relaxation processes, such that one can still neglect the vertex $\delta \Sigma$ for most of the time. Otherwise, one would have to compute a Bethe-Salpeter equation for the vertex on the Keldysh contour, which has been done for the ac-field-driven Falicov-Kimball model (Tsuji, Oka, and Aoki, 2009). In the most general case, solving the Bethe-Salpeter equation for a vertex that depends on four different time arguments is a formidable numerical task, so that it might be easier to compute the induced current $j$ for small probe fields directly from Eq. (110), and take the numerical derivative.

Neglecting the vertex, one obtains the following expressions (Eckstein and Kollar, 2008c) for the susceptibility:

$$
\chi_{\alpha \beta}^{\mathrm{pm}}\left(t, t^{\prime}\right)=-2 \chi_{0} \sum_{\boldsymbol{k} \sigma} v_{\boldsymbol{k}}^{\alpha}(t) v_{\boldsymbol{k}}^{\beta}\left(t^{\prime}\right) \operatorname{Im}\left[G_{\boldsymbol{k} \sigma}^{R}\left(t, t^{\prime}\right) G_{\boldsymbol{k} \sigma}^{<}\left(t^{\prime}, t\right)\right]
$$

$$
\chi_{\alpha \beta}^{\mathrm{dia}}\left(t, t^{\prime}\right)=-\chi_{0} \delta\left(t-t^{\prime}\right) \sum_{\boldsymbol{k} \sigma} \frac{\partial_{\boldsymbol{k}_{\alpha}} \partial_{\boldsymbol{k}_{\beta}} \epsilon_{\boldsymbol{k}}(t)}{\hbar} \operatorname{Im} G_{\boldsymbol{k} \sigma}^{<}(t, t)
$$

with $\chi_{0}=e^{2} / V \hbar$. Together with Eq. (121), they constitute the final DMFT expressions for the optical conductivity. In equilibrium, these equations reduce to the familiar relation (Pruschke, Cox, and Jarrell, 1993)

$$
\begin{aligned}
\operatorname{Re} \sigma_{\alpha \beta}(\omega) \stackrel{\omega>0}{=} & \pi \chi_{0} \sum_{\boldsymbol{k} \sigma} v_{\boldsymbol{k}}^{\alpha} v_{\boldsymbol{k}}^{\beta} \int_{-\infty}^{\infty} d \omega^{\prime} \\
& \times \frac{A_{\boldsymbol{k} \sigma}\left(\omega^{\prime}\right) A_{\boldsymbol{k} \sigma}\left(\omega+\omega^{\prime}\right)\left[f\left(\omega^{\prime}\right)-f\left(\omega+\omega^{\prime}\right)\right]}{\omega} .
\end{aligned}
$$

Even out of equilibrium, one can generally show from analytic properties of $\sigma(\omega, t)=\int d \bar{t} e^{i \omega \bar{t}} \sigma(t+\bar{t} / 2, t-\bar{t} / 2)$ that the nonequilibrium $f$ sum rule (Shimizu and Yuge, 2011; Tsuji, 2011)

$$
\begin{aligned}
\int_{0}^{\infty} d \omega \operatorname{Re} \sigma_{\alpha \beta}(\omega, t) & =\frac{\pi e^{2}}{2 V} \sum_{k \sigma} \frac{\partial_{\boldsymbol{k}_{\alpha}} \partial_{\boldsymbol{k}_{\beta}} \epsilon_{\boldsymbol{k}}(t)}{\hbar^{2}} n_{\boldsymbol{k} \sigma}(t) \\
& \equiv \frac{\pi}{2} \epsilon_{0} \omega_{p}^{2}(t)
\end{aligned}
$$

holds, which gives the familiar result for the plasma frequency $\omega_{p}^{2}=n e^{2} / \epsilon_{0} m^{*}$ of electrons near the bottom of a parabolic band $\epsilon_{\boldsymbol{k}}=\hbar^{2} \boldsymbol{k}^{2} / 2 m^{*}$. 


\section{Real-time impurity solvers}

\section{General remarks}

In this section we discuss methods to solve the nonequilibrium impurity models which play a central role in the DMFT formalism (Sec. II.B). A general impurity action defined on the L-shaped contour $\mathcal{C}$ (Fig. 4) has the form

$$
\begin{gathered}
\mathcal{S}_{\mathrm{imp}}=\mathcal{S}_{\mathrm{loc}}+\mathcal{S}_{\mathrm{hyb}} \\
\mathcal{S}_{\mathrm{loc}}=-i \int_{C} d t H_{\mathrm{loc}}\left[d_{p}^{\dagger}(t), d_{p}(t), t\right] \\
\mathcal{S}_{\mathrm{hyb}}=-i \int_{C} d t_{1} d t_{2} \sum_{p_{1}, p_{2}} d_{p_{1}}^{\dagger}\left(t_{1}\right) \Delta_{p_{1}, p_{2}}\left(t_{1}, t_{2}\right) d_{p_{2}}\left(t_{2}\right)
\end{gathered}
$$

Here $d_{p}$ and $d_{p}^{\dagger}$ denote, respectively, annihilation and creation operators for an electron in the impurity level $p$ ( $p$ labels spin and orbital degrees of freedom), and $H_{\text {loc }}$ is the local Hamiltonian of the impurity site, which can be interacting and time dependent in general. The hybridization function $\Delta_{p_{1}, p_{2}}\left(t_{1}, t_{2}\right)$ gives the amplitude for the hopping of an electron from the $p_{2}$ orbital into the bath at time $t_{2}$, its propagation within the bath, and the hopping back into the impurity orbital $p_{1}$ at time $t_{1}$. It is related to the Weiss Green's function $\mathcal{G}_{0, p_{1}, p_{2}}\left(t, t^{\prime}\right)$ in DMFT by

$$
\mathcal{G}_{0, p_{1}, p_{2}}^{-1}\left(t, t^{\prime}\right)=\left(i \partial_{t}+\mu\right) \delta_{\mathcal{C}}\left(t, t^{\prime}\right) \delta_{p_{1}, p_{2}}-\Delta_{p_{1}, p_{2}}\left(t, t^{\prime}\right) .
$$

The action (127) can be derived from an impurity Hamiltonian with a time-dependent coupling between the impurity and the bath

$$
\begin{gathered}
H_{\mathrm{imp}}(t)=H_{\mathrm{loc}}(t)+H_{\mathrm{bath}}(t)+H_{\mathrm{hyb}}(t), \\
H_{\mathrm{bath}}(t)=\sum_{\nu} \epsilon_{\nu}(t) c_{\nu}^{\dagger} c_{\nu}, \\
H_{\mathrm{hyb}}(t)=\sum_{p, \nu}\left[V_{p, \nu}(t) d_{p}^{\dagger} c_{\nu}+\text { H.c. }\right],
\end{gathered}
$$

by tracing out the bath degrees of freedom $c_{\nu}$. The hybridization function is given by

$$
\Delta_{p_{1}, p_{2}}\left(t, t^{\prime}\right)=\sum_{\nu} V_{p_{1}, \nu}(t) g_{\nu}\left(t, t^{\prime}\right) V_{p_{2}, \nu}\left(t^{\prime}\right)^{*}
$$

with

$$
g_{\nu}\left(t, t^{\prime}\right)=i\left[f\left(\epsilon_{\nu}(0)\right)-\theta_{C}\left(t, t^{\prime}\right)\right] e^{-i \int_{t^{\prime}}^{t} d \bar{t} \epsilon_{\nu}(\bar{t})}
$$

being the noninteracting bath Green's function without coupling to the impurity site.

By "solving the impurity problem," we essentially mean computing the single-particle Green's function of the impurity model (127)

$$
G_{p, p^{\prime}}\left(t, t^{\prime}\right)=-i\left\langle\mathcal{T}_{c} d_{p}(t) d_{p^{\prime}}^{\dagger}\left(t^{\prime}\right)\right\rangle_{S_{\text {imp }}}
$$

The nonequilibrium impurity solvers may be classified into two classes: diagrammatic approaches and Hamiltonian-based approaches. The former treat the impurity action (127) using diagrammatic techniques without direct reference to a given Hamiltonian formulation, while the latter solve the timedependent Hamiltonian (129) directly. In nonequilibrium DMFT calculations, the Hamiltonian approach requires one to reconstruct a given hybridization function $\Delta\left(t, t^{\prime}\right)$ by optimizing the (time-dependent) bath parameters $\epsilon_{\nu}(t)$ and $V_{p, \nu}(t)$, which is a nontrivial problem. The Hamiltonian approach has been used in combination with the numerical renormalization group (Joura, Freericks, and Pruschke, 2008), and very recently with the exact-diagonalization-based method (Arrigoni, Knap, and von der Linden, 2013; Gramsch et al., 2013). In the case of a steady state, the effective Matsubara method (Han and Heary, 2007) based on Hershfield's expression (Hershfield, 1993) for a steady-state density matrix has been tested as an impurity solver (Aron, Weber, and Kotliar, 2013). In the following, we focus on the diagrammatic approaches, which have been used in various types of applications.

\section{Falicov-Kimball model}

The Falicov-Kimball (FK) model [Eq. (83)] may be regarded as a simplified version of the Hubbard model, because only one electron species $\left(c, c^{\dagger}\right)$ can hop between lattice sites. The localized electrons $\left(f, f^{\dagger}\right)$ provide an annealed disorder potential, i.e., their equilibrium distribution is not governed by a fixed probability distribution (which would correspond to quenched disorder), but it is determined by statistical mechanics, which assumes that all $f$ configurations are in principle accessible and can contribute to the partition function. In nonequilibrium, however, the FK model is special because each $f_{i}^{\dagger} f_{i}$ is a constant of motion and therefore the $f$ electrons will maintain their initial distribution. The FK model is a useful starting point for DMFT studies, because the effective local DMFT action for the $c$ particles becomes quadratic. Thus the $c$-electron Green's function can be calculated exactly (Brandt and Mielsch, 1989; van Dongen and Vollhardt, 1990; van Dongen, 1992). In thermodynamic equilibrium, the model exhibits correlation-induced transitions between metallic, insulating, and charge-ordered phases (Freericks and Zlatić, 2003).

In nonequilibrium DMFT, the single-site action and the $c$-electron Green's function for the homogeneous phase are given by (Turkowski and Freericks, 2005; Freericks, Turkowski, and Zlatić, 2006)

$$
\begin{gathered}
S=-i \int_{C} d t d t^{\prime} c^{\dagger}(t) \Delta\left(t, t^{\prime}\right) c\left(t^{\prime}\right)-i \int_{C} d t U(t) n_{c}(t) n_{f}(t) \\
G\left(t, t^{\prime}\right)=-i \frac{\operatorname{Tr}_{c, f}\left[e^{-\beta H_{0}} \mathcal{T}_{c} e^{S} c(t) c^{\dagger}\left(t^{\prime}\right)\right]}{\operatorname{Tr}_{c, f}\left[e^{-\beta H_{0}} \mathcal{T}_{c} e^{S}\right]}
\end{gathered}
$$

Here $\mathcal{C}$ is the L-shaped contour in Fig. $4, n_{c}=c^{\dagger} c, n_{f}=$ $f^{\dagger} f$, and the operators are in the interaction representation with respect to $H_{0}=\left(E_{f}-\mu\right) f^{\dagger} f-\mu c^{\dagger} c$. The $f$ electrons can thus be traced out and the $c$ Green's function is obtained as 


$$
G\left(t, t^{\prime}\right)=w_{0} Q\left(t, t^{\prime}\right)+w_{1} R\left(t, t^{\prime}\right) .
$$

Here $w_{1}=\left\langle f^{\dagger} f\right\rangle=1-w_{0}$, and $Q\left(t, t^{\prime}\right)$ and $R\left(t, t^{\prime}\right)$ are defined as $G\left(t, t^{\prime}\right)$ in Eq. (133b) but without $\operatorname{Tr}_{f}$ and with $f^{\dagger}(\bar{t}) f(\bar{t})$ replaced by 0 and 1 , respectively, i.e., they are determined by the equations of motion

$$
\begin{gathered}
{\left[i \partial_{t}+\mu\right] Q\left(t, t^{\prime}\right)-(\Delta * Q)\left(t, t^{\prime}\right)=\delta_{C}\left(t, t^{\prime}\right),} \\
{\left[i \partial_{t}+\mu-U(t)\right] R\left(t, t^{\prime}\right)-(\Delta * R)\left(t, t^{\prime}\right)=\delta_{C}\left(t, t^{\prime}\right),}
\end{gathered}
$$

with an antiperiodic boundary condition (see Sec. II.A.1.b). These equations must be solved together with the selfconsistency condition (see Sec. II.B). The solution for abrupt and slow interaction changes is discussed in Secs. III.B.1 and III.B.2.

\section{Continuous-time quantum Monte Carlo algorithms \\ a. General remarks}

A general strategy for evaluating expectation values such as Eq. (132) is to write $H_{\text {imp }}$ as a sum of two terms: one, $H_{1}$, for which the time evolution can be computed exactly and another, $H_{2}$, which is treated by a formal perturbative expansion. The expansion in $H_{2}$ generates a series of diagrams which are sampled stochastically, using an importance sampling which accepts or rejects proposed diagrams on the basis of their contributions to the partition function of the initial state. We discuss two types of expansions: In the weak coupling method, $H_{\text {imp }}$ is split into a quadratic part $H_{0}$ and an interacting part $H_{\text {int }}$, and the expansion is performed in terms of $H_{\text {int }}$. In the strong coupling approach, $H_{\text {loc }}$ and $H_{\text {bath }}$ are treated exactly, while $H_{\text {hyb }}$ is treated as a perturbation.

These so-called continuous-time quantum Monte Carlo (CTQMC) algorithms provide very efficient and flexible solvers for equilibrium quantum impurity problems at temperature $T>0$ [for a review, see Gull, Millis et al. (2011)]. In equilibrium the expansion can be formulated on the imaginary time branch, and at least for single-site impurity problems with diagonal bath, the fermionic sign problem can be avoided. In the nonequilibrium extension of these methods (Mühlbacher and Rabani, 2008; Werner, Oka, and Millis, 2009), the expansion must also be performed on the real-time branches of the contour $\mathcal{C}$, where the convergence of the perturbation theory is oscillatory rather than monotonic. The weights of the diagrams become complex, and the resulting dynamical sign problem limits the time range over which accurate results can be obtained.

For nonequilibrium DMFT applications, the times which can be reached with the strong-coupling CTQMC approach are usually too short. We introduce this formalism mainly to set the stage for more useful approximate strong-coupling methods (see Sec. II.C.5). The weak-coupling CTQMC method, on the other hand, thanks to a simplification of the diagrammatic structure in half-filled single-band systems, has proven useful in situations where the interesting phenomena happen on a fast time scale and where a numerically exact treatment, e.g., of the intermediate correlation regime, is essential. This method has enabled some pioneering nonequilibrium DMFT studies of the Hubbard model (Eckstein, Kollar, and Werner, 2009; Tsuji et al., 2011).

\section{b. Weak-coupling CTQMC}

We first discuss the implementation of the weak-coupling continuous-time Monte Carlo algorithm (Rubtsov, Savkin, and Lichtenstein, 2005; Gull et al., 2008; Werner, Oka, and Millis, 2009; Werner et al., 2010) for the Anderson impurity model

$$
H_{\mathrm{loc}}(t)=U(t)\left(n_{\uparrow}-\alpha_{\uparrow}\right)\left(n_{\downarrow}-\alpha_{\downarrow}\right)-\sum_{\sigma}\left[\mu-U(t) \alpha_{\bar{\sigma}}\right] n_{\sigma},
$$

where a spin-dependent chemical potential shift $\left[U(t) \alpha_{\bar{\sigma}}\right]$ has been introduced to avoid a trivial sign problem in the repulsively interacting case (Rubtsov, Savkin, and Lichtenstein, 2005) with $\bar{\sigma}=\downarrow$, $\uparrow$ for $\sigma=\uparrow, \downarrow$. The impurity action (127) is split into an interaction term

$$
S_{\mathrm{int}}=-i \int_{\mathcal{C}} d t H_{\mathrm{int}}(t)=-i \int_{\mathcal{C}} d t U(t)\left(n_{\uparrow}-\alpha_{\uparrow}\right)\left(n_{\downarrow}-\alpha_{\downarrow}\right),
$$

and the rest $S_{0}=S_{\mathrm{imp}}-S_{\mathrm{int}}$, which is quadratic in the fermionic operators. Expanding the partition function $Z=$ $\operatorname{Tr}\left[\mathcal{T}_{c} e^{S_{\text {imp }}}\right]=\operatorname{Tr}\left[\mathcal{T}_{C} e^{S_{0}+S_{\text {int }}}\right]$ in powers of $S_{\text {int }}$ leads to

$$
Z=\sum_{n=0}^{\infty} \frac{(-i)^{n}}{n !} \int_{C} d t_{1} \ldots \int_{C} d t_{n} \operatorname{Tr}\left[\mathcal{T}_{C} e^{s_{0}} H_{\mathrm{int}}\left(t_{1}\right) \ldots H_{\mathrm{int}}\left(t_{n}\right)\right]
$$

After separating the trace into spin-up and spin-down factors and using the definition (32), we obtain

$$
\begin{aligned}
\frac{Z}{Z_{0}}= & \sum_{n=0}^{\infty} \frac{(-i)^{n}}{n !} \int_{\mathcal{C}} d t_{1} \cdots \int_{\mathcal{C}} d t_{n} U\left(t_{1}\right) \cdots U\left(t_{n}\right) \prod_{\sigma}\left\langle\left[ n_{\sigma}\left(t_{1}\right)\right.\right. \\
& \left.\left.-\alpha_{\sigma}\right] \cdots\left[n_{\sigma}\left(t_{n}\right)-\alpha_{\sigma}\right]\right\rangle_{S_{0}}
\end{aligned}
$$

with $Z_{0}=\operatorname{Tr}\left[\mathcal{T}_{c} e^{S_{0}}\right]$. Wick's theorem allows one to express the expectation values in Eq. (138) as the determinant of an $n \times n$ matrix $M_{\sigma}^{-1}$, with elements

$$
\begin{aligned}
& \left(M_{\sigma}^{-1}\right)_{i, j}=-i \mathcal{G}_{0, \sigma}\left(t_{i}, t_{j}\right)-\alpha_{\sigma} \delta_{i, j}, \\
& \mathcal{G}_{0, \sigma}\left(t, t^{\prime}\right)=-i\left\langle\mathcal{T}_{C} d_{\sigma}(t) d_{\sigma}^{\dagger}\left(t^{\prime}\right)\right\rangle_{S_{0}},
\end{aligned}
$$

and the convention $\mathcal{G}_{0, \sigma}(t, t) \equiv \mathcal{G}_{0, \sigma}^{<}(t, t)$ (note that these functions depend on the choice of $\alpha_{\sigma}$ ). Equation (138) therefore expresses $Z / Z_{0}=\sum_{c} w(c)$ as a sum over configurations $c=\left\{t_{1} \prec t_{2} \prec \cdots \prec t_{n}\right\}$, which are collections of time points on the contour $\mathcal{C}$, with a weight

$$
w(c)=(-i)^{n}\left[U\left(t_{1}\right) d t_{1}\right] \cdots\left[U\left(t_{n}\right) d t_{n}\right] \prod_{\sigma} \operatorname{det} M_{\sigma}^{-1}
$$


Here $d t_{i}=d t$ for $t_{i}$ on the forward branch $\mathcal{C}_{1},-d t$ for $t_{i}$ on the backward branch $\mathcal{C}_{2}$, and $-i d \tau$ for $t_{i}$ on the imaginary-time branch $\mathcal{C}_{3}$ (Fig. 4).

A Monte Carlo sampling over all configurations $c$ can then be implemented on the basis of these weights (Werner, Oka, and Millis, 2009). The determinants in Eq. (141) sum up all the $n$ ! connected and disconnected bare diagrams for a given set of $n$ interaction vertices. An example of such a diagram is shown in the top panel of Fig. 8. While this summation (for a proper choice of the $\alpha_{\sigma}$ ) absorbs the sign cancellations originating from Fermi statistics, the weights $w(c)$ are in general complex, so the sampling suffers from a phase problem (dynamical sign problem). This phase problem grows exponentially with the number of interaction vertices on the real-time branches, and hence the length of the real-time contour (i.e., $\langle$ phase $\rangle \sim C e^{-\alpha t_{\max }}$ ).

In a practical implementation, one generates a Markov chain of configurations $c_{1} \rightarrow c_{2} \rightarrow c_{3} \rightarrow \cdots$ in such a way that every configuration can be visited from any other within a finite number of steps (ergodicity) and that the probability for the configuration to change from $c_{i}$ to $c_{j}\left[p\left(c_{i} \rightarrow c_{j}\right)\right]$ satisfies the detailed balance condition

$$
p\left(c_{i} \rightarrow c_{j}\right)\left|w\left(c_{i}\right)\right|=p\left(c_{j} \rightarrow c_{i}\right)\left|w\left(c_{j}\right)\right| .
$$

This guarantees that the configuration $c$ is realized with a probability $\propto|w(c)|$. To sample the configurations, one
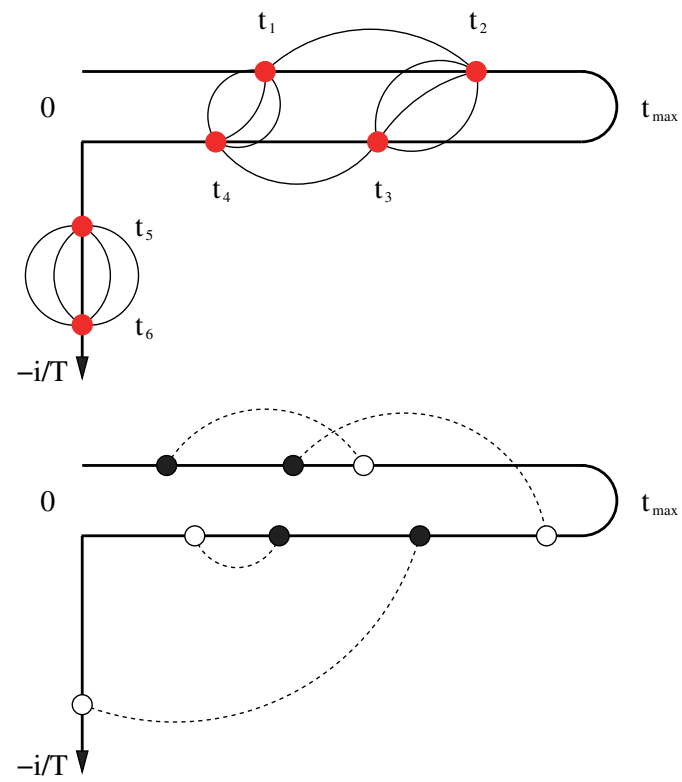

FIG. 8 (color online). Illustration of the CTQMC method. Top panel: Example of a sixth-order diagram appearing in the weakcoupling expansion. Interaction vertices are linked by bath Green's functions $\mathcal{G}_{0}$. Bottom panel: Example of a fourth-order diagram appearing in the strong coupling expansion: full (empty) dots represent impurity creation (annihilation) operators. Pairs of creation and annihilation operators are linked by hybridization functions $\Delta$ (dashed lines). In both methods, all diagrams obtained by linking a given collection of operators by Green's functions or hybridization lines are summed up into a determinant. usually adopts the Metropolis-Hastings algorithm (Metropolis et al., 1953; Hastings, 1970). One proposes to insert an $n$th interaction vertex at $t_{n} \in \mathcal{C}$ with probability $p^{\text {prop }}(n-1 \rightarrow n)=\left|d t_{n}\right| /\left(2 t_{\max }+\beta\right)$, or to remove the $n$th vertex with probability $p^{\operatorname{prop}}(n \rightarrow n-1)=1 / n$. Then one accepts the proposed insertion (removal) with the probability $\min \left[1, R^{(n)}\right]\left(\min \left[1, R^{(n)-1}\right]\right)$, where

$$
\begin{aligned}
R^{(n)} & =\frac{p^{\operatorname{acc}}(n-1 \rightarrow n)}{p^{\operatorname{acc}}(n \rightarrow n-1)}=\frac{p^{\operatorname{prop}}(n \rightarrow n-1)|w(n)|}{p^{\operatorname{prop}}(n-1 \rightarrow n)|w(n-1)|} \\
& =\left|U\left(t_{n}\right)\right| \frac{2 t_{\max }+\beta}{n} \prod_{\sigma} \frac{\left|\operatorname{det}\left(M_{\sigma}^{(n)}\right)^{-1}\right|}{\left|\operatorname{det}\left(M_{\sigma}^{(n-1)}\right)^{-1}\right|}
\end{aligned}
$$

The ratio of two determinants in Eq. (143) can be obtained by a fast-update algorithm with a computational cost of $O\left(n^{2}\right)$ (Gull, Millis et al., 2011).

To measure an observable by the Monte Carlo sampling, $\langle O(t)\rangle_{\mathrm{MC}}$, we perform a similar expansion in the presence of the operator $O$ at time $t$. The measurement formula becomes

$$
\langle O(t)\rangle_{\mathrm{MC}}=\frac{\sum_{c} w_{c} \frac{O_{c}}{w_{c}}}{\sum_{c} w_{c}}=\frac{\sum_{c}\left|w_{c}\right| \text { phase }_{c} \frac{O_{c}}{w_{c}}}{\sum_{c}\left|w_{c}\right| \text { phase }_{c}},
$$

where $O_{c}$ is the weight corresponding to the collection $c$ of operators in the presence of $O(t)$, and phase ${ }_{c}=w_{c} /\left|w_{c}\right|$. In the case of the Green's function (132), the ratio $O_{c} / w_{c}$ (or rather the analog for two-time operators) becomes $\mathcal{G}_{0, \sigma}\left(t, t^{\prime}\right)+i \sum_{i, j=1}^{n} \mathcal{G}_{0, \sigma}\left(t, t_{i}\right)\left(M_{\sigma}\right)_{i, j} \mathcal{G}_{0, \sigma}\left(t_{j}, t^{\prime}\right)$, and the measurement formula reads

$$
\begin{aligned}
G_{\sigma}\left(t, t^{\prime}\right)= & \mathcal{G}_{0, \sigma}\left(t, t^{\prime}\right)+\int_{\mathcal{C}} d s_{1} \int_{\mathcal{C}} d s_{2} \mathcal{G}_{0, \sigma}\left(t, s_{1}\right) \mathcal{G}_{0, \sigma}\left(s_{2}, t^{\prime}\right) \\
& \times\left\langle i \sum_{i, j=1}^{n} \delta_{\mathcal{C}}\left(s_{1}, t_{i}\right)\left(M_{\sigma}\right)_{i, j} \delta_{\mathcal{C}}\left(s_{2}, t_{j}\right)\right\rangle_{\mathrm{MC}} \cdot
\end{aligned}
$$

It is therefore sufficient to accumulate the quantity (improper self-energy) (Gull et al., 2008; Werner et al., 2010)

$$
X_{\sigma}\left(s_{1}, s_{2}\right)=\left\langle i \sum_{i, j=1}^{n} \delta_{C}\left(s_{1}, t_{i}\right)\left(M_{\sigma}\right)_{i, j} \delta_{C}\left(s_{2}, t_{j}\right)\right\rangle_{\mathrm{MC}} .
$$

Comparison of Eq. (145) to the Dyson equation shows that $X$ is related to the (proper) self-energy $\Sigma$ by $X * \mathcal{G}_{0}=\Sigma * G$, so the measurement of $X$ allows one to extract $\Sigma$. This equation is a Volterra integral equation of the first kind (45), for which numerical solutions are known to be unstable. We may, however, combine it with the Dyson equation $G_{\sigma}=\mathcal{G}_{0, \sigma}+$ $G_{\sigma} * \Sigma_{\sigma} * \mathcal{G}_{0, \sigma}$ to find

$$
\left(1+X_{\sigma} * \mathcal{G}_{0, \sigma}\right) * \Sigma_{\sigma}=X_{\sigma}
$$

This equation is a Volterra equation of the second kind (44) with unknown $G=\Sigma$ and kernel $F=X_{\sigma} * \mathcal{G}_{0, \sigma}$, which can be solved in the same way as the Dyson equation (Sec. II.B.2).

Let us briefly mention the relationship of the above algorithm to the continuous-time auxiliary field method (Gull et al., 2008; Werner, Oka, and Millis, 2009). In practice, 
it is advantageous to symmetrize the $\alpha_{\sigma}$, which amounts to introducing an Ising spin degree of freedom at every vertex position. For example, up to irrelevant constants we may write the interaction term $U n_{\uparrow} n_{\downarrow}$ in the form (Assaad and Lang, 2007)

$$
\frac{U(t)}{2} \sum_{s= \pm 1}\left(n_{\uparrow}-\frac{1}{2}-s \delta\right)\left(n_{\downarrow}-\frac{1}{2}+s \delta\right)+\frac{U(t)}{2}\left(n_{\uparrow}+n_{\downarrow}\right),
$$

and absorb the last (quadratic term) into $\mathcal{G}_{0}$. The configuration space then becomes the space of all Ising spin configurations on the contour $\mathcal{C}: c=\left\{\left(t_{1}, s_{1}\right),\left(t_{2}, s_{2}\right), \ldots,\left(t_{n}, s_{n}\right)\right\}$. To avoid a fermionic sign problem in the repulsively interacting case (away from half filling) one has to choose $\delta>1 / 2$. At half filling, one should use $\delta=0$, i.e., the symmetric form $H_{\text {int }}=U(t)\left(n_{\uparrow}-\frac{1}{2}\right)\left(n_{\downarrow}-\frac{1}{2}\right)$. In this case, all odd-order diagrams vanish, which leads to a less severe sign problem. The interaction expansion based on Eq. (148) is equivalent to the auxiliary field algorithms described by Gull et al. (2008) and Werner, Oka, and Millis (2009), as was shown by Mikelsons, Macridin, and Jarrell (2009).

\section{c. Strong-coupling CTQMC}

A complementary diagrammatic Monte Carlo algorithm can be obtained by performing an expansion in powers of the hybridization term $H_{\text {hyb }}$ (Mühlbacher and Rabani, 2008; Schiró and Fabrizio, 2009; Werner, Oka, and Millis, 2009). We sketch here the derivation for the general impurity model defined in Eq. (129a). In the hybridization expansion approach the time evolution of the operators is given by $H_{\text {loc }}+H_{\text {bath }}$, and the starting point is the identity

$$
Z=\operatorname{Tr}\left[\mathcal{T}_{C} e^{-i \int_{C} d s\left[H_{\mathrm{loc}}(s)+H_{\mathrm{bath}}(s)\right]-i \int_{C} d s H_{\mathrm{hyb}}(s)}\right]
$$

Expanding the contour-ordered exponential into a power series yields

$$
\begin{aligned}
Z= & \operatorname{Tr}\left[\mathcal{T}_{C} e^{-i \int_{C} d s\left[H_{\mathrm{loc}}(s)+H_{\mathrm{bath}}(s)\right]}\right. \\
& \left.\times \sum_{n=0}^{\infty} \frac{(-i)^{n}}{n !} \int_{\mathcal{C}} d t_{1} \cdots \int_{\mathcal{C}} d t_{n} H_{\mathrm{hyb}}\left(t_{1}\right) \cdots H_{\mathrm{hyb}}\left(t_{n}\right)\right] .
\end{aligned}
$$

We now proceed in exactly the same way as in equilibrium (Werner and Millis, 2006; Werner et al., 2006), namely, separate the $H_{\text {hyb }}$ factors into impurity creation terms (time arguments $t_{i}^{\prime}$ ) and impurity annihilation terms (time arguments $t_{j}$ ). Then, because the bath is noninteracting, we can evaluate the trace $\operatorname{Tr}_{\text {bath }}[\cdots]$ over the bath Hilbert space exactly. Wick's theorem yields a determinant $\operatorname{det} N^{-1}$, with the size of the matrix $N^{-1}$ given by the number of impurity creation (or annihilation) operators on the contour $\mathcal{C}$, and with matrix elements given by the hybridization functions $\Delta$ :

$$
\left(N^{-1}\right)_{i, j}=\Delta_{p_{i}^{\prime}, p_{j}}\left(t_{i}^{\prime}, t_{j}\right)
$$

Here $t_{i}^{\prime}$ denotes the position of the $i$ th creation operator and $t_{j}$ the position of the $j$ th annihilation operator, with flavor $p_{i}$ and $p_{j}$, respectively. The weight of a Monte Carlo configuration with $n$ creation and $n$ annihilation operators on the contour then becomes

$$
\begin{aligned}
& w\left(\left\{\left(t_{1}^{\prime}, p_{1}^{\prime}\right), \ldots,\left(t_{n}^{\prime}, p_{n}^{\prime}\right) ;\left(t_{1}, p_{1}\right), \ldots,\left(t_{n}, p_{n}\right)\right\}\right) \\
& =\operatorname{Tr}_{\mathrm{loc}}\left[\mathcal{T}_{c} e^{-i \int_{c} d s H_{\mathrm{loc}}(s)} d_{p_{1}^{\prime}}^{\dagger}\left(t_{1}^{\prime}\right) d_{p_{1}}\left(t_{1}\right) \cdots d_{p_{n}^{\prime}}^{\dagger}\left(t_{n}^{\prime}\right) d_{p_{n}}\left(t_{n}\right)\right] \\
& \quad \times d t_{1} \cdots d t_{n}^{\prime} \frac{(-1)^{n}}{(n !)^{2}} \operatorname{det} N^{-1}
\end{aligned}
$$

where the $d t_{i}$ again contains factors $+1,-1$, or $-i$, depending on the branch corresponding to $t_{i}$. The trace over the impurity states, $\operatorname{Tr}_{\text {loc }}[\cdots]$, is calculated explicitly, for example, by expressing the impurity creation and annihilation operators, as well as the time evolution operators

$$
U\left(t_{2}, t_{1}\right)=\mathcal{T}_{C} e^{-i \int_{t_{1}}^{t_{2}} H_{\mathrm{loc}}(s) d s}
$$

as matrices in the eigenbasis of the Hamiltonian $H_{\text {loc }}(0)$. This is particularly simple if the eigenbasis of $H_{\text {loc }}$ is the occupation number basis, and if the eigenbasis does not change in time (as is the case, for example, for the Anderson impurity model with a time-dependent interaction term). Monte Carlo configurations are then generated stochastically by insertion and removal of pairs of impurity creation and annihilation operators, based on the absolute value of the weight (152).

The interpretation of the determinant of the $n \times n$ hybridization matrix $N^{-1}$ is that it sums all strong-coupling diagrams which can be obtained for a given collection of $n$ creation and $n$ annihilation operators, by connecting pairs of these operators by hybridization functions $\Delta$; see the bottom panel of Fig. 8 .

The Green's function can be measured by removing a single hybridization line, in complete analogy to the equilibrium case (Werner et al., 2006). This leads to a measurement procedure which accumulates delta functions with weights given by the matrix elements of $(N)_{j, i}$ at times $t_{i}^{\prime}$ and $t_{j}$. Particularly simple is the measurement of local observables $O(t)$, such as the density or double occupancy. They can be measured by inserting the corresponding operator into the trace factor $\operatorname{Tr}_{\text {loc }}[\cdots]$.

\section{Weak-coupling perturbation theory}

The phase problem of CTQMC techniques can be avoided if subclasses of diagrams are summed up analytically using Dyson's equation (38). In this approach, the object of interest is the self-energy, whose diagrammatic expansion is truncated at some given order. We discuss here the weak-coupling perturbation theory for the Anderson impurity model with the local Hamiltonian defined in Eq. (135).

Perturbation theory for nonequilibrium impurity problems (Hershfield, Davies, and Wilkins, 1991, 1992; Fujii and Ueda, 2003) is a straightforward generalization of the equilibrium perturbation theory formulated on the Matsubara branch (Yosida and Yamada, 1970; Abrikosov, Gorkov, and Dzyaloshinski, 1975; Yamada, 1975, Yosida and Yamada, 
1975; Mahan, 2000). The weak-coupling perturbation theory has been used for a long time as an impurity solver in equilibrium DMFT calculations (Georges and Kotliar, 1992; Zhang, Rozenberg, and Kotliar, 1993; Freericks, 1994; Freericks and Jarrell, 1994; Georges et al., 1996), and in the nonequilibrium DMFT context, it has enabled a range of studies of the Hubbard model (Schmidt and Monien, 2002; Heary and Han, 2009; Eckstein, Kollar, and Werner, 2010; Eckstein and Werner, 2011a; Amaricci et al., 2012; Aron, Kotliar, and Weber, 2012; Tsuji et al., 2012; Tsuji and Werner, 2013; Tsuji, Eckstein, and Werner, 2013) and of the FalicovKimball model (Turkowski and Freericks, 2007a).

We again split the impurity action into a quadratic term $S_{0}$ and an interaction term (136), and expand $\exp \left(\mathcal{S}_{\text {imp }}\right)=$ $\exp \left(\mathcal{S}_{0}+\mathcal{S}_{\text {int }}\right)$ in Eq. (132) into a Taylor series with respect to the interaction term

$$
\begin{aligned}
G_{\sigma}\left(t, t^{\prime}\right)= & (-i) \frac{1}{Z} \sum_{n=0}^{\infty} \frac{(-i)^{n}}{n !} \int_{C} d t_{1} \cdots d t_{n} \\
& \times \operatorname{Tr}\left[\mathcal{T}_{c} e^{S_{0}} H_{\mathrm{int}}\left(t_{1}\right) \cdots H_{\mathrm{int}}\left(t_{n}\right) d_{\sigma}(t) d_{\sigma}^{\dagger}\left(t^{\prime}\right)\right] .
\end{aligned}
$$

The linked cluster theorem ensures that all disconnected diagrams that contribute to Eq. (153) can be factorized to give a proportionality constant $Z / Z_{0}$ with $Z_{0}=\operatorname{Tr}\left[\mathcal{T}_{c} e^{S_{0}}\right]$. As a result, the expansion of the Green's function may be written as

$$
\begin{aligned}
G_{\sigma}\left(t, t^{\prime}\right)= & (-i) \sum_{n=0}^{\infty}(-i)^{n} \int_{\mathcal{C}, t_{1} \prec \cdots \prec t_{n}} d t_{1} \cdots d t_{n} \\
& \times\left\langle\mathcal{T}_{C} H_{\mathrm{int}}\left(t_{1}\right) \cdots H_{\mathrm{int}}\left(t_{n}\right) d_{\sigma}(t) d_{\sigma}^{\dagger}\left(t^{\prime}\right)\right\rangle_{S_{0}}^{\mathrm{conn}} .
\end{aligned}
$$

where "conn" means that we only consider the connected diagrams. The factor $n$ ! has been cancelled by specifying the contour ordering as $t_{1} \prec \cdots \prec t_{n}$. Owing to Wick's theorem, one can evaluate each term in Eq. (154) using the bath Green's function (140).

In the weak-coupling perturbation theory, one usually considers an expansion of the self-energy $\Sigma_{\sigma}\left(t, t^{\prime}\right)$ instead of the Green's function. This allows one to generate an infinite series of diagrams for the Green's function by solving the Dyson equation (38). The self-energy consists of the oneparticle irreducible parts of the expansion (154). We show topologically distinct Feynman diagrams of the self-energy up to third order in Fig. 9. Because of the chemical potential shift $\mu \rightarrow \mu-U(t) \alpha_{\bar{\sigma}}$ in Eq. (135), a tadpole diagram with a bold or bare line amounts to $n_{\bar{\sigma}}-\alpha_{\bar{\sigma}}$ or $n_{0 \bar{\sigma}}-\alpha_{\bar{\sigma}}$ respectively, where $n_{\sigma}(t)=-i G_{\sigma}^{<}(t, t)$ and $n_{0 \sigma}(t)=-i \mathcal{G}_{0 \sigma}^{<}(t, t)$.

The Feynman rules to calculate the self-energy diagrams on the contour $\mathcal{C}$ are as follows: (1) Draw topologically distinct one-particle irreducible diagrams. (2) Associate each solid line [bold line] with the Weiss Green's function $(-i) \mathcal{G}_{0 \sigma}\left(t, t^{\prime}\right)$ [the interacting Green's function $(-i) G_{\sigma}\left(t, t^{\prime}\right)$ ]. (3) Multiply $(-i) U(t)$ for each interaction vertex (dashed line). (4) Multiply $n_{\sigma}(t)-\alpha_{\sigma}\left[n_{0 \sigma}(t)-\alpha_{\sigma}\right]$ for each bold [bare] tadpole diagram. (5) Multiply $(-1)$ for each Fermion loop. (6) Multiply an additional factor $(-i)$, coming from the definition of the Green's function (132). (7) Carry out a (a)

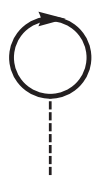

(c)

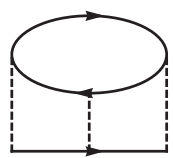

(b)

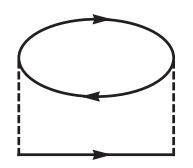

(d)

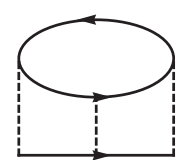

FIG. 9. The self-energy diagrams up to third order. The thin lines represent $\mathcal{G}_{0 \sigma}\left(t, t^{\prime}\right)$, the bold line $G_{\sigma}\left(t, t^{\prime}\right)$, and the dashed lines the interaction vertices.

contour integral along $\mathcal{C}$ for each internal vertex. For example, the first-order diagram [the Hartree term, Fig. 9(a)] is given by

$$
\Sigma_{\sigma}^{(1)}\left(t, t^{\prime}\right)=U(t)\left[n_{\bar{\sigma}}(t)-\alpha_{\bar{\sigma}}\right] \delta_{\mathcal{C}}\left(t, t^{\prime}\right),
$$

and the second-order diagram [Fig. 9(b)] by

$$
\Sigma_{\sigma}^{(2)}\left(t, t^{\prime}\right)=U(t) U\left(t^{\prime}\right) \mathcal{G}_{0 \sigma}\left(t, t^{\prime}\right) \mathcal{G}_{0 \bar{\sigma}}\left(t^{\prime}, t\right) \mathcal{G}_{0 \bar{\sigma}}\left(t, t^{\prime}\right)
$$

At half filling in the paramagnetic phase, it is natural to choose $\alpha_{\sigma}=\frac{1}{2}$. This cancels all the tadpole diagrams since $n_{\sigma}-\alpha_{\sigma}=n_{0 \sigma}-\alpha_{\sigma}=0$, and due to the particle-hole symmetry [i.e., $\mathcal{G}_{0 \sigma}\left(t, t^{\prime}\right)=-\mathcal{G}_{0 \sigma}\left(t^{\prime}, t\right)$ ], all the odd-order diagrams vanish as well. On the other hand, away from half filling or in a spin-polarized phase (whenever $n_{\uparrow} \neq n_{\downarrow}$ ), it is nontrivial how to deal with the Hartree and other tadpole diagrams. One may take bold diagrams [such as Fig. 9(a)] with the interacting density $n_{\sigma}$, which is self-consistently determined, or bare diagrams with the noninteracting density $n_{0 \sigma}$. One can also explicitly expand the Hartree diagram with respect to the interaction up to a given order. For the antiferromagnetic phase at half filling, the best accuracy is attained when one takes $\alpha_{\sigma}=\frac{1}{2}$ and expands all the bold loops in the tadpole diagrams up to the same order as the rest of the self-energy (Tsuji and Werner, 2013; Tsuji, Eckstein, and Werner, 2013).

One issue with the expansion of $\Sigma$ into bare diagrams is that the resulting perturbation theory is not a conserving approximation. As a consequence, the total energy of the system will drift with increasing time, even if the Hamiltonian is time independent. In DMFT simulations of the Hubbard model, one finds that this drift is very small for weak-to-intermediate coupling ( $U \lesssim U_{c} / 2$, with $U_{c}$ the critical value for the Mott transition in the paramagnetic phase). The drift saturates at a certain time scale, so that the bare perturbation theory can be trusted up to very long time for these $U$ (Tsuji and Werner, 2013). A similar behavior is found when second-order perturbation theory is used to study the Hubbard model driven by an electric field: For small $U$, energy conservation in the form of Eq. (115) is satisfied for long times (Eckstein and Werner, 2011a).

For stronger coupling, the perturbation theory can however fail rapidly and quite abruptly. This contrasts with the equilibrium case, where the second-order perturbation theory accidentally reproduces the correct strong-coupling limit at half-filling, gives a reasonable interpolation between the weak- and the strong-coupling regime, and even captures the Mott transition (Zhang, Rozenberg, and Kotliar, 1993; 

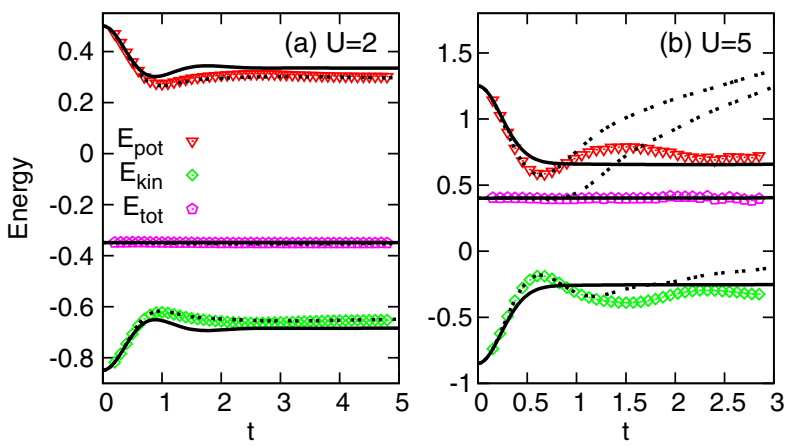

FIG. 10 (color online). Nonequilibrium DMFT results for the kinetic, potential, and total energies of the Hubbard model after an interaction quench from $U=0$ to $U=2$ (left panel) and $U=5$ (right panel). Symbols show the weak-coupling CTQMC result, dashed lines the result from bare second-order perturbation theory, and the solid lines the result from self-consistent second-order perturbation theory. Adapted from Eckstein, Kollar, and Werner, 2010.

Georges et al., 1996). A simple way to fix the problem with the drifting energy might seem to switch to self-consistent perturbation theory. Here one replaces the bare propagators $\mathcal{G}_{0}$ in the self-energy diagrams by bold propagators $G$, and considers only the diagrams which are two-particle irreducible. However, even though this approximation is conserving, it reproduces the time evolution of the system very poorly (Eckstein, Kollar, and Werner, 2010; Tsuji and Werner, 2013). Figure 10 shows the kinetic and potential energy, as well as the total energy after a quench in the Hubbard model (semicircular density of states, bandwidth 4), from a noninteracting initial state to $U=2$ (left panel) and $U=5$ (right panel). Selfconsistent perturbation theory conserves the total energy, but gives wrong values for the kinetic and potential energies, already after a very short time. While bare second-order perturbation theory fails for the quench to $U=5$, it at least reproduces the short-time dynamics correctly. For driven steady states (Amaricci et al., 2012), the accuracy of the weak-coupling approach has yet to be tested in detail. Here the nonconserving nature of the approximation becomes apparent when the energy current to the reservoir differs from the power injected by the field [Eq. (115)].

\section{Strong-coupling perturbation theory}

\section{a. General remarks}

The strong-coupling CTQMC approach (Sec. II.C.3.c) sums all diagrams generated by a hybridization expansion on the contour $\mathcal{C}$. While the summation into a determinant allows one to absorb some of the sign cancellations between these diagrams, the Monte Carlo weights are complex, and the resulting phase problem restricts simulations to relatively short times. To avoid this sign problem, one can analytically sum certain subsets of the strong-coupling diagrams using a Dyson equation. This approach is expected to work well in the strongcoupling regime, in particular, the Mott insulating phase, where the hybridization can be treated as a perturbation.

The lowest order perturbative strong-coupling method is called the noncrossing approximation (NCA), because it sums all diagrams without crossing hybridization lines. It was originally proposed as an approximate solution for the Anderson impurity model (Keiter and Kimball, 1971; Grewe and Keiter, 1981; Kuramoto, 1983), where it gives a reasonable description of the physics down to the Kondo temperature $T_{K}$, but fails in the Fermi-liquid regime for $T \ll$ $T_{K}$ (Müller-Hartmann, 1984). The deficiencies of NCA are partly cured by higher order summations (Pruschke and Grewe, 1989; Haule et al., 2001). In particular, the simplest extension of NCA, the so-called one-crossing approximation (OCA) (Pruschke and Grewe, 1989), largely corrects the underestimation of $T_{K}$ of the NCA at $U<\infty$. From early on (Pruschke, Cox, and Jarrell, 1993) until today [see, e.g., Shim, Haule, and Kotliar (2007a, 2007b)], both the NCA and the OCA have been used as impurity solvers for DMFT. In the context of nonequilibrium DMFT, an attractive feature of the perturbative strong-coupling expansion is its conserving nature, and its good convergence properties with increasing order of the approximation in the Mott phase (Eckstein and Werner, 2010).

Within any approximation to the strong-coupling expansion, one must resum parts of the series to infinite order to avoid severe artifacts and make the theory conserving. There exist various derivations of a resummed strong-coupling expansion (Keiter and Kimball, 1971; Barnes, 1976; Grewe and Keiter, 1981; Kuramoto, 1983; Coleman, 1984; Bickers, 1987; Bickers, Cox, and Wilkins, 1987) that all circumvent the problem that Wick's theorem does not apply, because the unperturbed action is not quadratic. A detailed derivation of the strong-coupling equations on the Keldysh contour in the context of nonequilibrium DMFT (Eckstein and Werner, 2010) employed the pseudoparticle technique (Coleman, 1984) [for earlier real-time formulations and applications of the NCA, see Nordlander et al. (1999) and Okamoto (2008)]. Here we provide an alternative derivation of the same equations, which builds on the strong-coupling CTQMC formalism introduced in Sec. II.C.3.c.

\section{b. Self-consistent strong-coupling equations}

The starting point for NCA and its extensions is a Taylor expansion of the action (127) in terms of the hybridization $\Delta$, analogous to Eq. (150). To resum terms of Eq. (127) to infinite order, one must decouple trace terms like $\operatorname{Tr}\left[\mathcal{T}_{c} e^{S_{\mathrm{loc}}} d_{p_{1}}^{\dagger}\left(t_{1}\right) d_{p_{2}}\left(t_{2}\right) \cdots\right]$. Wick's theorem does not apply, but for any given collection of the times $t_{1}, t_{2}, \ldots$ along $\mathcal{C}$, one can insert a complete set of states of the impurity Hilbert space, $\sum_{n}|n\rangle\langle n|$, between consecutive operators, and thus factor the trace into a matrix product of impurity propagators $g$ and hybridization vertices $F^{p}$ and $\bar{F}^{p} \equiv\left(F^{p}\right)^{\dagger}$ :

$$
\begin{gathered}
g_{n m}\left(t, t^{\prime}\right) \stackrel{t \succ t^{\prime}}{=}-i\left\langle n\left|\mathcal{T}_{C} e^{-i \int_{t^{\prime}}^{t} d \bar{t} H_{\mathrm{loc}}(\bar{t})}\right| m\right\rangle, \\
F_{n m}^{p}=\left\langle n\left|d_{p}\right| m\right\rangle, \quad \bar{F}_{n m}^{p}=\left\langle n\left|d_{p}^{\dagger}\right| m\right\rangle .
\end{gathered}
$$

The factor $-i$ in $g$ is inserted for convenience. For example, $\operatorname{Tr}\left[\mathcal{T}_{c} e^{S_{\mathrm{loc}}} d_{p}^{\dagger}(t) d_{q}\left(t^{\prime}\right)\right]=-i \operatorname{Tr}\left[g(-i \beta, t) \bar{F}^{p} g\left(t, t^{\prime}\right) F^{q} g\left(t^{\prime}, 0\right)\right]$ for $t \succ t^{\prime}$, and $i \operatorname{Tr}\left[g\left(-i \beta, t^{\prime}\right) F^{q} g\left(t^{\prime}, t\right) \bar{F}^{p} g(t, 0)\right]$ for $t^{\prime} \succ t$. With a suitable graphical representation of vertices $F$ and propagators $g$ [Fig. 11(a)], one can represent the Taylor expansion 


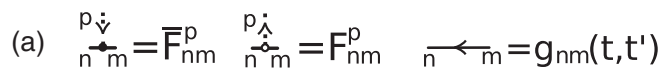

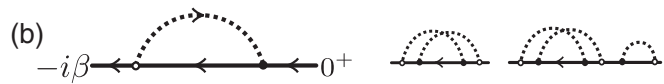

(c)

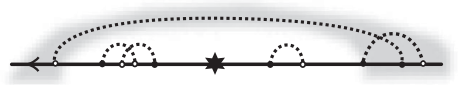

(d)

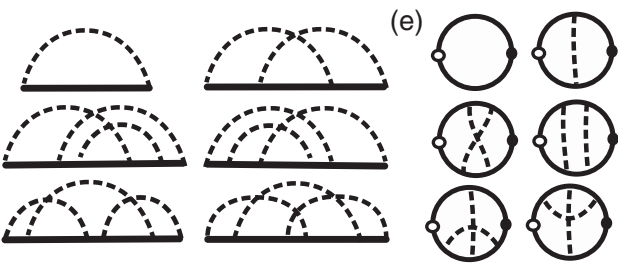

FIG. 11. Elements of the strong-coupling expansion: (a) vertices [Eq. (158)] and lines [Eq. (157)]. (b) Some diagrams for $Z$, in the open-contour representation (from $0^{+}$on the upper branch of $\mathcal{C}$, to $-i \beta)$. (c) Diagram for $\langle\mathcal{O}(t)\rangle$ (star) in the open-contour representation. The shaded part can be viewed as an initial-state correction, or lesser self-energy. (d) All skeleton diagrams $\mathfrak{S}[\mathcal{G}, \Delta]$ up to third order. The first two diagrams define the NCA and OCA, respectively. (e) All skeleton diagrams $G[\mathcal{G}, \Delta]$ up to third order.

of $Z[\Delta]$ as a sum of diagrams constructed according to the following rules: (i) The $n$th order contribution to $Z[\Delta]$ is given by all topologically inequivalent diagrams consisting of one sequence of $2 n+1$ directed lines $g$ ("backbone"), separated by $2 n$ three-leg vertices ( $n$ annihilation events $F, n$ creation events $\bar{F}$ ), which are connected by $n$ (directed) hybridization lines in all possible ways. (ii) External time arguments $(0$ and $-i \beta)$ and internal times are contour ordered, $-i \beta \succ t_{2 n} \succ \cdots \succ t_{1} \succ 0$. Perform the trace over the product of $g$ and $F$ factors, sum over all internal flavor indices, and integrate over the internal times (keeping the contour ordering). (iii) The sign of the diagram is $(-1)^{s+f}$, where $s$ is the number of crossings of hybridization lines, and $f$ is the number of hybridization lines that point opposite to the direction of the backbone. (iv) An overall factor $i^{n+1}$ is added. For example, the expression for the simplest diagram in Fig. 11(b) is

$$
\begin{aligned}
& \sum_{p_{1}, p_{2}} \int_{-i \beta \succ t_{2} \succ t_{1} \succ 0} d t_{2} d t_{1} \operatorname{Tr}\left[g\left(-i \beta, t_{2}\right) F^{p_{2}} g\left(t_{2}, t_{1}\right) \bar{F}^{p_{1}} g\left(t_{1}, 0\right)\right] \\
& \quad \times \Delta_{p_{1} p_{2}}\left(t_{1}, t_{2}\right)
\end{aligned}
$$

where the sign is $-(-i)^{2}=+1$.

To resum the series, one can define an impurity level selfenergy $\mathfrak{S}_{n m}\left(t, t^{\prime}\right)$ as the sum of all parts of the above diagrams that cannot be separated into two by cutting a single $g$ line, without the outer trace and the outer two $g$ factors, and with an overall factor $i^{n}$ instead of $i^{n+1}$. For example, the selfenergy part in the first diagram in Fig. 11(b) is $\mathfrak{S}\left(t, t^{\prime}\right)=$ $-i \sum_{p, q} F^{p} g\left(t, t^{\prime}\right) \bar{F}^{q} \Delta_{q p}\left(t^{\prime}, t\right)$. One can then introduce renormalized propagators $\mathcal{G}$ via the Dyson equation,

$$
\mathcal{G}=g+g(\Im * \mathcal{G}=g+\mathcal{G} * \Im * g,
$$

where, for $t \succ t^{\prime}, \circledast$ denotes the convolution $\int_{\mathcal{C}} d \bar{t} a(t, \bar{t}) b\left(\bar{t}, t^{\prime}\right)$ restricted to contour-ordered time arguments $t \succ \bar{t} \succ t^{\prime}$. Since there are no symmetry factors associated with the diagrams, the partition function is given by

$$
Z=i \operatorname{Tr}\left[\mathcal{G}\left(-i \beta, 0^{+}\right)\right]
$$

Observables in the initial state can simply be evaluated by computing $\langle\mathcal{O}(0)\rangle=i \operatorname{Tr}[\mathcal{O} \mathcal{G}(-i \beta, 0)] / Z$, where $\mathcal{O}$ is the matrix with elements $\langle n|\mathcal{O}| m\rangle$. For time $t>0$, however, a diagram for $\langle\mathcal{O}(t)\rangle$ in general contains initial-state vertex corrections [shaded part of Fig. 11(c)], i.e., it is not proportional to $\operatorname{Tr}[\mathcal{G}(-i \beta, t) \mathcal{O} \mathcal{G}(t, 0)]$. To obtain a consistent description of the self-energy $\mathbb{S}$ and these initial-state corrections, one can show that the latter can be viewed as the "lesser" part of $\mathfrak{S}$, i.e., a self-energy $\mathfrak{S}\left(t, t^{\prime}\right)$ with $t \prec t^{\prime}$. Since spectral functions and occupations are no longer related for conventional nonequilibrium Green's functions, one must separately compute the lesser $\left(t \prec t^{\prime}\right)$ and retarded $\left(t \succ t^{\prime}\right)$ components of $\mathfrak{S}\left(t, t^{\prime}\right)$ and $\mathcal{G}\left(t, t^{\prime}\right)$ within the nonequilibrium strong-coupling expansion. To be precise, we first define the lesser component of the bare propagators (157):

$$
g\left(t, t^{\prime}\right)=-i \xi g\left(t, 0^{+}\right) g\left(-i \beta, t^{\prime}\right) \quad \text { for } \quad t \prec t^{\prime},
$$

i.e., the time evolution is performed in clockwise order along $\mathcal{C}$, from $t^{\prime}$ to $-i \beta$ and then from 0 to $t$. For later convenience a sign $\xi$ is included in the definition: $\xi$ is a diagonal matrix, with $\xi_{m}=-1(+1)$ when the number of particles in $|m\rangle$ is odd and even, respectively, $\left([\xi, g]=[\xi, \mathcal{G}]=0\right.$, because $H_{\text {loc }}$ conserves the particle number). One can then define the lesser selfenergy by the same diagram rules as before, and $\mathcal{G}$ by the Dyson equation (159), only extending the contour-ordering constraint in the time integrals to clockwise order

$$
\begin{aligned}
& {[a * b]\left(t, t^{\prime}\right)} \\
& \quad= \begin{cases}\int_{\mathcal{C}, t^{\prime}}^{t} d \bar{t} a(t, \bar{t}) b\left(\bar{t}, t^{\prime}\right) & t \succ t^{\prime} \\
\int_{\mathcal{C}, t^{\prime}}^{-i \beta} d \bar{t} a(t, \bar{t}) b\left(\bar{t}, t^{\prime}\right)+\int_{\mathcal{C}, 0^{+}}^{t} d \bar{t} a(t, \bar{t}) b\left(\bar{t}, t^{\prime}\right) & t \prec t^{\prime} .\end{cases}
\end{aligned}
$$

These definitions for $g, \mathcal{G}$, and $\mathfrak{\subseteq}$ are actually equivalent to the projected pseudoparticle propagators used in the alternative formulation (Eckstein and Werner, 2010). We can now write the partition function in terms of the lesser propagators

$$
\begin{aligned}
Z & =i \operatorname{Tr}\left[\xi \mathcal{G}\left(t^{+}, t^{-}\right)\right], \\
\langle\mathcal{O}(t)\rangle & =i \frac{1}{Z} \operatorname{Tr}\left[\mathcal{O} \xi \mathcal{G}\left(t^{+}, t^{-}\right)\right],
\end{aligned}
$$

where $t^{ \pm}$is a time on the upper (lower) branch of $\mathcal{C}$. The equality of Eqs. (163) and (160) can be verified most easily order by order: A given term in the expansion of $\mathcal{G}\left(t^{+}, t^{-}\right)$can be mapped onto a term of the expansion of $\mathcal{G}(-i \beta, 0)$ by using Eq. (161) and cyclically permuting operators under the trace. The change of the sign of the diagram, associated with the number of $\Delta$ lines which are flipped with respect to the $g$ lines, is accounted for by the $\xi$ factors in Eqs. (161) and (163). 
Approximations for $\mathcal{G}, Z$, and any observable are obtained by truncating the series for $\mathfrak{S}$. Since there are no symmetry factors in the diagrams for $\mathfrak{S}$, one can formally reproduce all terms in the series by replacing $g$ lines in a diagram by fully renormalized $\mathcal{G}$ lines, and in turn omitting all diagrams in which the lines have self-energy insertions (skeleton diagrams). All skeleton diagrams up to third order are shown in Fig. 11(d). The truncation of this skeleton series at a given order defines a conserving approximation. More generally, an approximation is conserving when $\mathfrak{S}[\mathcal{G}, \Delta]$ is a derivative $\delta \Phi / \delta \mathcal{G}\left(t^{\prime}, t\right)$ of some functional $\Phi$, where the exact $\Phi$ is the Luttinger-Ward functional. While it is not a priori clear that conserving approximations are necessarily better than nonconserving ones, the perturbative strong-coupling impurity solvers which have been tested so far are self-consistent approximations which are conserving and satisfy $\sum_{n}|n\rangle\langle n|=1$ at any time.

The most important observable for DMFT is the Green's function

$$
G_{p p^{\prime}}\left(t, t^{\prime}\right)=-\frac{1}{Z} \frac{\delta Z[\Delta]}{\delta \Delta_{p^{\prime} p}\left(t^{\prime}, t\right)}
$$

One can derive diagrammatic rules for $G$ and reformulate them in terms of fully interacting propagators $\mathcal{G}$ similar to time-local observables (164). Again, it is convenient not to deal with initial-state correlations but instead close the contour at $-i \beta$ and $0^{+}$and include lesser components. The following rules for $G$ result: (i) The $n$th order contribution consists of all topologically inequivalent loops of $2 n \mathcal{G}$ lines which connect $n$ $F$-vertices and $n \bar{F}$-vertices, where one of each type is an external vertex. The internal vertices are connected by $\Delta$ lines such that no $\mathcal{G}$ line has a self-energy insertion. (ii) Sum over all internal flavor indices, and integrate over times, respecting their clockwise order. (iii) To determine the sign of a diagram, reinsert the $\Delta$ line between the external vertices, open the loop at any point, insert the sign $\xi$, and add the factor $(-1)^{s+f}$, where $s$ is the number of crossings of hybridization lines, and $f$ is the number of hybridization lines that point opposite to the $g$ lines. (iv) Add a prefactor $i^{n}$.

It can be shown that $G$ is the derivative of the Luttinger-Ward functional $G\left(t, t^{\prime}\right)=(-1 / Z) \delta \Phi[\Delta, \mathcal{G}] / \delta \Delta\left(t^{\prime}, t\right)$ (at fixed $\mathcal{G}$ ). Hence consistent self-consistent diagrammatic expansions for $\mathfrak{\subseteq}$ and $G$ are obtained by choosing one approximation to the Luttinger-Ward functional. All skeleton diagrams for $\mathfrak{\Im}$ and $G$ up to third order are shown in Figs. 11(d) and 11(e).

\section{c. Numerical implementation}

Within DMFT, one numerically computes the integrals for S (lesser and greater components), solves the Dyson equation (159), computes the diagrams for $G$, and feeds the result into the self-consistency (II.B.4) to update $\Delta$. The numerical effort is mainly determined by the contour integrals over the internal vertices in the evaluation of the diagrams, and scales like $N^{3}, N^{4}$, and $N^{5}$ for the first, second, and third order, respectively [where $N$ is the number of time steps (Eckstein and Werner, 2010)]. For solving the Dyson equation (159) one can switch to a differential notation, starting from the equation of motion for bare propagators, Eqs. (157) and (161),

$$
\begin{gathered}
{\left[i \partial_{t}-H_{\mathrm{loc}}(t)\right] g\left(t, t^{\prime}\right)=0,} \\
g\left(t, t^{\prime}\right)\left[-i \overleftarrow{\partial}_{t^{\prime}}-H_{\mathrm{loc}}\left(t^{\prime}\right)\right]=0,
\end{gathered}
$$

which must be solved with the initial condition $g\left(t^{-}, t^{+}\right)=$ $-i$. Applying the term in brackets to Eq. (159), one obtains two equivalent integral-differential equations for $\mathcal{G}$

$$
\begin{aligned}
& {\left[i \partial_{t}-H_{\mathrm{loc}}(t)\right] \mathcal{G}\left(t, t^{\prime}\right)-[\Im \circledast \mathcal{G}]\left(t, t^{\prime}\right)=0,} \\
& \mathcal{G}\left(t, t^{\prime}\right)\left[-i \overleftarrow{\partial}_{t^{\prime}}-H_{\mathrm{loc}}\left(t^{\prime}\right)\right]-[\mathcal{G} * \Im]\left(t, t^{\prime}\right)=0
\end{aligned}
$$

which must be solved for $t \neq t^{\prime}$ with the initial condition $\mathcal{G}\left(t^{-}, t^{+}\right)=-i$. Although this is an initial-value problem instead of a boundary-value problem (even on the Matsubara branch), and integrals are time ordered, this equation is a causal contour equation like Eqs. (40) [more details are given in Eckstein and Werner (2010)]. Hence, the whole set of nonequilibrium DMFT equations can again be implemented in the form of a time-propagation scheme.

Results up to third order, for the time evolution of the double occupancy after an interaction quench in the Hubbard model (DMFT calculation for a semicircular density of states with bandwidth 4), are shown in Fig. 12. For not too low temperatures (this is often the relevant regime in experiments, such as pump-probe experiments which strongly excite the electrons), one finds a good convergence of the results with increasing order. [Note that in the correlated metal phase $\left(U_{0}=3\right)$, NCA cannot provide an adequate description of the initial equilibrium phase.]
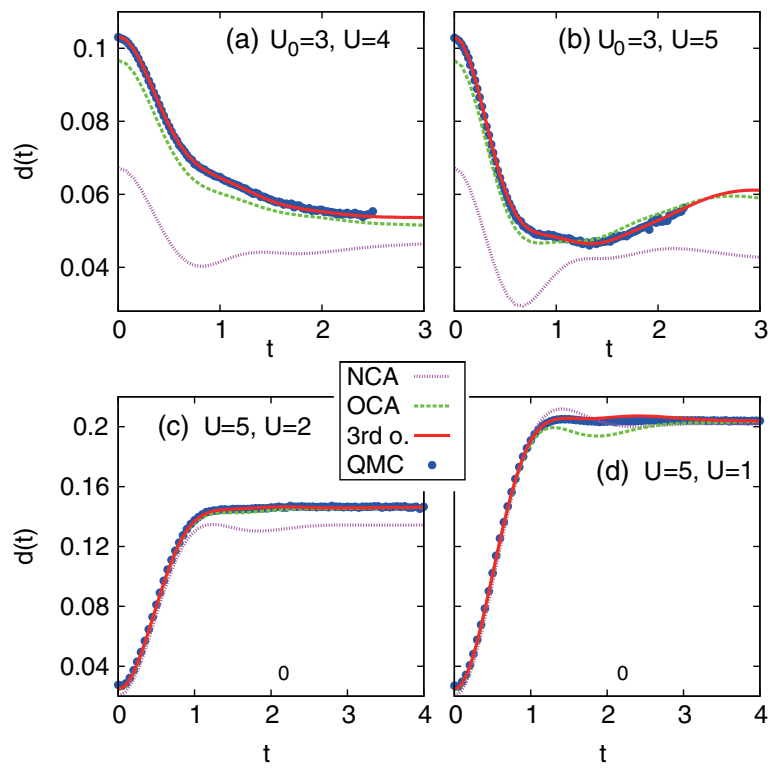

FIG. 12 (color online). Nonequilibrium DMFT results for the time evolution of the double occupancy in the Hubbard model after a quench from $U(t=0)=U_{0}$ to $U$. The symbols show weak-coupling CTQMC results, and the lines results from strong-coupling perturbation theory. From Eckstein and Werner, 2010. 


\section{d. Monte Carlo sampling around NCA}

A numerically exact Monte Carlo method based on NCA (or OCA) propagators has been described by Gull, Reichman, and Millis (2010). The idea here is to stochastically sample all strong-coupling diagrams using a worm-type Monte Carlo algorithm (Prokof'ev, Svistunov, and Tupitsyn, 1998). Since the building blocks of the diagrams are NCA propagators, the number of diagrams is reduced with respect to the strongcoupling CTQMC method, and longer times can be reached. The method is particularly suitable for the study of insulating phases, where NCA is a good starting point and the corrections from crossing diagrams are small. In the metallic regime, where diagrams with complicated topologies become relevant, the sampling of individual bold-line diagrams (instead of summing up collections of bare diagrams into a determinant) leads to a fermionic sign problem, in addition to the dynamical sign problem. In practice, all contributing bold-line diagrams are summed up at factorial cost to reduce this problem.

Applications of this method to nonequilibrium quantum impurity models have been presented by Gull, Reichman, and Millis (2011). While it has not yet been used in the context of nonequilibrium DMFT, such an application, in appropriate parameter regimes, seems promising. The Monte Carlo sampling around NCA or higher-order approximations could also become a useful tool to estimate the errors accumulated in perturbative strong-coupling calculations.

The method has also been used to evaluate the memory function of the Nakajima-Zwanzig-Mori equation (Nakajima, 1958; Zwanzig, 1960; Mori, 1965), a quantum master equation for the reduced density matrix of the impurity problem, which enables one to compute the time evolution of the reduced density matrix up to longer times (Cohen and Rabani, 2011; Cohen et al., 2013).

\section{Floquet formalism for periodically driven systems}

When a quantum system is continuously driven by a timeperiodic external force, it may enter a nonequilibrium steady state in which the overall time dependence of the system is periodic. For example, the pump pulse in ultrafast pump-probe experiments may be viewed (during irradiation) as a timeperiodic ac electric field if the laser pulse contains many oscillation cycles. A theoretical approach to treat periodically driven states is the Floquet method (Shirley, 1965; Ritus, 1966; Zel'dovich, 1966; Sambe, 1973; Dittrich et al., 1998; Grifoni and Hänggi, 1998). It originates from Floquet's theorem (Floquet, 1883; Hill, 1886; Magnus and Winkler, 1966), a temporal analog of Bloch's theorem for a spatially periodic system. Because of the periodicity of external fields, the timedependent problem can be mapped onto a time-independent eigenvalue problem. Recently, the Floquet method has been employed in combination with DMFT to study nonequilibrium steady states of periodically driven correlated systems (Schmidt and Monien, 2002; Freericks and Joura, 2008; Joura, Freericks, and Pruschke, 2008; Tsuji, Oka, and Aoki, 2008; Lubatsch and Kroha, 2009; Tsuji, Oka, and Aoki 2009). An advantage of the Floquet DMFT is that one does not have to calculate the full time evolution until a nonequilibrium steady state is reached. It suffices to solve small-size matrix equations for nonequilibrium Green's functions represented in frequency space, which greatly reduces the computational cost.

The Floquet formalism has been used in the study of Floquet topological insulators (Oka and Aoki, 2009b; Kitagawa et al., 2011; Lindner, Refael, and Galitski, 2011). The topology of quantum systems can be controlled by external time-periodic perturbations; for example, by applying circularly polarized light to graphene (or other many-band systems), one can change a trivial state into a quantum Hall insulator (Oka and Aoki, 2009b; Kitagawa et al., 2011). The methods which we describe here can be applied to such problems.

\section{Overview of Floquet's theorem}

Floquet's theorem (Floquet, 1883; Hill, 1886; Magnus and Winkler, 1966) is a general statement about the solution of an ordinary differential equation $d x(t) / d t=C(t) x(t)$ with timeperiodic coefficients $C(t)$. Here we apply the theorem to the time-dependent Schrödinger equation

$$
i \frac{d}{d t} \Psi(t)=H(t) \Psi(t)
$$

where the Hamiltonian $H(t)$ is assumed to be periodic in time with period $\mathscr{T}, H(t+\mathscr{T})=H(t)$. Floquet's theorem states that there exists a solution of Eq. (170) of the form

$$
\Psi_{\alpha}(t)=e^{-i \varepsilon_{\alpha} t} u_{\alpha}(t)
$$

where $u_{\alpha}(t)=u_{\alpha}(t+\mathscr{T})$ is a periodic function of $t$, and the real number $\varepsilon_{\alpha}$ is called the quasienergy, which is unique up to integer multiples of $\Omega=2 \pi / \mathscr{T}$. To prove this, we write the formal solution of Eq. (170) as

$$
\Psi(t)=\mathcal{V}\left(t, t_{0}\right) \Psi\left(t_{0}\right),
$$

with the time evolution operator $\mathcal{V}\left(t, t_{0}\right)=\mathcal{T} e^{-i \int_{t_{0}}^{t} d \bar{t} H(\bar{t})}$. Then we consider the operator

$$
\mathcal{V}\left(t+\mathscr{T}, t_{0}\right)=\mathcal{V}\left(t+\mathscr{T}, t_{0}+\mathscr{T}\right) \mathcal{V}\left(t_{0}+\mathscr{T}, t_{0}\right)
$$

which is split into two via the chain rule. The first part is $\mathcal{V}\left(t+\mathscr{T}, t_{0}+\mathscr{T}\right)=\mathcal{V}\left(t, t_{0}\right)$ due to the periodicity of the Hamiltonian. The second part $\mathcal{V}\left(t_{0}+\mathscr{T}, t_{0}\right)$ is called the Floquet operator, which we can write in terms of a Hermitian operator $Q\left(t_{0}\right)$ :

$$
e^{-i Q\left(t_{0}\right) \mathscr{T}} \equiv \mathcal{V}\left(t_{0}+\mathscr{T}, t_{0}\right)
$$

Multiplying both sides of Eq. (173) with $e^{i Q\left(t_{0}\right)(t+\mathscr{T})}$ from the right, we thus have

$$
\mathcal{V}\left(t+\mathscr{T}, t_{0}\right) e^{i Q\left(t_{0}\right)(t+\mathscr{T})}=\mathcal{V}\left(t, t_{0}\right) e^{i Q\left(t_{0}\right) t},
$$

which shows that the unitary operator $P\left(t, t_{0}\right) \equiv$ $\mathcal{V}\left(t, t_{0}\right) e^{i Q\left(t_{0}\right) t}$ is periodic in $t$, i.e., $P\left(t+\mathscr{T}, t_{0}\right)=P\left(t, t_{0}\right)$. The solution (172) of the Schödinger equation (170) has thus been written in terms of a ( $t$-independent) Hermitian operator $Q\left(t_{0}\right)$ and a unitary time-periodic operator $P\left(t, t_{0}\right)$ :

$$
\Psi(t)=P\left(t, t_{0}\right) e^{-i Q\left(t_{0}\right) t} \Psi\left(t_{0}\right)
$$


When the initial state $\Psi\left(t_{0}\right)$ is an eigenstate of $Q\left(t_{0}\right)$ [denoted by $\left.\Psi_{\alpha}\left(t_{0}\right)\right]$ with eigenvalue $\varepsilon_{\alpha}$, the solution is given by Eq. (171) with $u_{\alpha}(t)=P\left(t, t_{0}\right) \Psi_{\alpha}\left(t_{0}\right)$. One can see that $u_{\alpha}(t)$ is a periodic function $\left[u_{\alpha}(t+\mathscr{T})=u_{\alpha}(t)\right]$, and that the quasienergy spectrum $\varepsilon_{\alpha}$ does not depend on $t_{0}$.

To determine $\varepsilon_{\alpha}$, one can Fourier expand $u_{\alpha}(t)$ as $u_{\alpha}(t)=$ $\sum_{n} e^{-i n \Omega t} u_{\alpha}^{n}$ (with $\Omega=2 \pi / \mathscr{T}$ ), where $u_{\alpha}^{n}$ is called the $n$th Floquet mode of the Floquet state (171). Then Eq. (170) gives

$$
\sum_{n}\left(H_{m n}-n \Omega \delta_{m n}\right) u_{\alpha}^{n}=\varepsilon_{\alpha} u_{\alpha}^{m},
$$

where

$$
H_{m n} \equiv \frac{1}{\mathscr{T}} \int_{0}^{\mathscr{T}} d t e^{i(m-n) \Omega t} H(t)
$$

is the Floquet matrix form of the Hamiltonian. Thus the quasienergies $\varepsilon_{\alpha}$ are the eigenvalues of the infinite-dimensional Floquet matrix $H_{m n}-n \Omega \delta_{m n}$. Note that if $\varepsilon_{\alpha}$ is an eigenvalue of $H_{m n}-n \Omega \delta_{m n}$, the same holds for $\varepsilon_{\alpha}+n \Omega$, for arbitrary integer $n$. To avoid the redundancy in $\varepsilon_{\alpha}$, we impose a condition that $-\Omega / 2<\varepsilon_{\alpha} \leq \Omega / 2$. As a consequence of the Floquet theorem, the time-dependent differential equation (170) has been transformed into a time-independent eigenvalue problem (177), which can be solved by simple linear algebra (if one truncates the matrix size).

\section{Floquet Green's function method}

\section{a. General formulation}

We now apply the Floquet method to the nonequilibrium Green's function formalism (Sec. II.A.2), as introduced earlier by Faisal (1989), Althorpe et al. (1997), Brandes and Robinson (2002), Martinez (2003, 2005), and Martinez and Molina (2006). In general, Green's functions $G^{R, A, K}\left(t, t^{\prime}\right)$ in the Keldysh formalism (Sec. II.A.2) have two independent time arguments $t, t^{\prime} \in(-\infty,+\infty)$ (two-time representation), or equivalently, relative time $t_{\text {rel }} \equiv t-t^{\prime}$ and averaged time $t_{\text {av }} \equiv\left(t+t^{\prime}\right) / 2$ (Wigner, 1932). We say that a periodically driven system has reached a nonequilibrium steady state (NESS) when its Green's functions become periodic as a function of $t_{\mathrm{av}}$, i.e.,

$$
G^{R, A, K}\left(t+\mathscr{T}, t^{\prime}+\mathscr{T}\right)=G^{R, A, K}\left(t, t^{\prime}\right) .
$$

The Floquet Green's function formalism (and Floquet DMFT) can describe such NESSs not only for the unitary evolution of isolated systems as considered in Sec. II.D.1 but also for the dissipative evolution of open systems coupled to environment. For the latter, one can determine the Floquet Green's function without considering the earlier transient dynamics. Mathematically, it is hard to prove that a driven system approaches a NESS in the long-time limit (in the same way as it is difficult to prove "thermalization" for an isolated system). However, for a dissipative system that is continuously driven by a time-periodic perturbation, NESS solutions obtained by the Floquet method usually exist and are unique, so that one may simply assume that this state is indeed

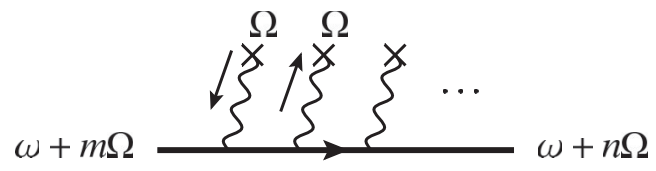

FIG. 13. Diagrammatic representation of the Floquet Green's function $\boldsymbol{G}_{m n}(\omega)$. The wavy lines denote photon propagators of the pump light, and the arrows indicate the energy flow.

established after the dependence on the initial condition is wiped out in the presence of dissipation.

For a Green's function $G\left(t, t^{\prime}\right)$ that satisfies the periodicity condition (179), we first define the Wigner representation (Wigner, 1932) by a Fourier transformation with respect to relative time

$$
G\left(\omega, t_{\mathrm{av}}\right)=\int_{-\infty}^{\infty} d t_{\mathrm{rel}} e^{i \omega t_{\mathrm{rel}}} G\left(t, t^{\prime}\right)
$$

From this we introduce the Floquet matrix form of $G$ :

$$
\boldsymbol{G}_{m n}(\omega) \equiv \frac{1}{\mathscr{T}} \int_{0}^{\mathscr{T}} d t_{\mathrm{av}} e^{i(m-n) \Omega t_{\mathrm{av}}} G\left(\omega+\frac{m+n}{2} \Omega, t_{\mathrm{av}}\right) .
$$

In this Floquet representation, we use the reduced zone scheme to avoid degeneracies, i.e., the range of $\omega$ is restricted to a "Brillouin zone" $-\Omega / 2<\omega \leq \Omega / 2$. To reconstruct $\boldsymbol{G}_{m n}(\omega)$ outside the Brillouin zone, one can shift the frequency with an integer $l$ such that $-\Omega / 2<\omega-l \Omega \leq \Omega / 2$, using the symmetry relation $\boldsymbol{G}_{m, n}(\omega)=\boldsymbol{G}_{m+l, n+l}(\omega-l \Omega)$. In Fig. 13, we show a diagrammatic representation of $\boldsymbol{G}_{m n}(\omega)$, a fermion propagator with multiple photon absorption or emission (external wavy lines). As a fermion propagates in the presence of the driving field, its energy changes from $\omega+m \Omega$ to $\omega+n \Omega$.

For time-periodic NESSs, the two-time representation, the Floquet representation, and the Wigner representation for Green's functions are equivalent. The relations among these three representations are summarized in Table II. The advantage of the Floquet representation is that a convolution in the two-time representation (or a Moyal product in the Wigner representation) can be translated into a simple matrix product in the Floquet representation. This greatly simplifies the solution of the Dyson equation (38), since the problem is reduced to a matrix inversion in the Floquet representation, and one can usually truncate the Floquet matrix to a relatively small size as long as higher-order nonlinear processes (multiphoton absorption or emission) are suppressed.

To further establish the formalism, we start from the retarded Green's function for a noninteracting system, which satisfies the Dyson equation (or equation of motion)

$$
\left[i \partial_{t}-H(t)\right] G^{R}\left(t, t^{\prime}\right)=\delta\left(t-t^{\prime}\right)
$$

where $H$ is the single-particle Hamiltonian, and all objects are regarded as matrices in orbital indices. A solution of Eq. (182) is explicitly given in terms of the Floquet wave functions $\Psi_{\alpha}(t)=e^{-i \varepsilon_{\alpha} t} u_{\alpha}(t)[$ Eq. (171)] as

$$
G_{\alpha}^{R}\left(t, t^{\prime}\right)=-i \theta\left(t-t^{\prime}\right) \Psi_{\alpha}(t) \Psi_{\alpha}^{\dagger}\left(t^{\prime}\right)
$$


TABLE II. Three representations of nonequilibrium Green's functions.

\begin{tabular}{lcc}
\hline \hline Two-time representation & Wigner representation & Floquet representation \\
\hline$G\left(t, t^{\prime}\right)=\int_{-\infty}^{\infty} \frac{d \omega}{2 \pi} e^{-i \omega t_{\mathrm{rel}}} G\left(\omega, t_{\mathrm{av}}\right)$ & $G\left(\omega, t_{\mathrm{av}}\right)=\int_{-\infty}^{\infty} d t_{\mathrm{rel}} e^{i \omega t_{\mathrm{rel}}} G\left(t, t^{\prime}\right)$ & $\boldsymbol{G}_{m n}(\omega)=\frac{1}{\mathscr{T}} \int_{0}^{\mathscr{T}} d t_{\mathrm{av}} e^{i(m-n) \Omega t_{\mathrm{av}}} G\left(\omega+\frac{m+n}{2} \Omega, t_{\mathrm{av}}\right)$ \\
$=\sum_{m n} \int_{-\Omega / 2}^{\Omega / 2} \frac{d \omega}{2 \pi} e^{-i(\omega+m \Omega) t+i(\omega+n \Omega) t^{\prime}} \boldsymbol{G}_{m n}(\omega)$ & $=\sum_{m-n} e^{-i(m-n) \Omega t_{\mathrm{av}}} \boldsymbol{G}_{m n}\left(\omega-\frac{m+n}{2} \Omega\right)$ & $=\frac{1}{\mathscr{T}} \int_{0}^{\mathscr{T}} d t_{\mathrm{av}} \int_{-\infty}^{\infty} d t_{\mathrm{rel}} e^{i(\omega+m \Omega) t-i(\omega+n \Omega) t^{\prime}} G\left(t, t^{\prime}\right)$ \\
Convolution: $(A * B)\left(t, t^{\prime}\right)$ & Moyal product: $(A \star B)\left(\omega, t_{\mathrm{av}}\right)$ & Matrix product: $(\boldsymbol{A} \cdot \boldsymbol{B})_{m n}(\omega)$ \\
$=\int_{-\infty}^{\infty} d \bar{t} A(t, \bar{t}) B\left(\bar{t}, t^{\prime}\right)$ & $=A\left(\omega, t_{\mathrm{av}}\right) \exp \left(\frac{i}{2}\left[\overleftarrow{\partial}_{\omega} \vec{\partial}_{t_{\mathrm{av}}}\right.\right.$ & $=\sum_{l} \boldsymbol{A}_{m l}(\omega) \boldsymbol{B}_{l n}(\omega)$ \\
& $\left.\left.-\vec{\partial}_{t_{\mathrm{av}}} \vec{\partial}_{\omega}\right]\right) B\left(\omega, t_{\mathrm{av}}\right)$ & \\
\hline \hline
\end{tabular}

Since $\Psi_{\alpha}(t+\mathscr{T})=e^{-i \varepsilon_{\alpha} \mathscr{T}} \Psi_{\alpha}(t)$, the Green's function (183) manifestly satisfies the periodicity condition (179). Its Floquet representation is given by (Tsuji, Oka, and Aoki, 2008)

$$
\boldsymbol{G}_{\alpha}^{R}(\omega)=\boldsymbol{U}_{\alpha} \cdot \boldsymbol{Q}_{\alpha}(\omega) \cdot \boldsymbol{U}_{\alpha}^{\dagger}
$$

with a unitary matrix

$$
\left(\boldsymbol{U}_{\alpha}\right)_{m n}=\frac{1}{\mathscr{T}} \int_{0}^{\mathscr{T}} d t e^{i(m-n) \Omega t} u_{\alpha}(t),
$$

and a diagonal matrix

$$
\left(\boldsymbol{Q}_{\alpha}\right)_{m n}(\omega)=\frac{1}{\omega+n \Omega+\mu-\varepsilon_{\alpha}+i \eta} \delta_{m n} .
$$

Thus, $\boldsymbol{G}_{\alpha}^{R}(\omega)$ has poles at the Floquet quasienergies $\omega=\varepsilon_{\alpha}-n \Omega \quad(n=0, \pm 1, \pm 2, \ldots)$. For a noninteracting single-band system described by the Hamiltonian $H_{0}(t)=$ $\sum_{k} \epsilon_{k}(t) c_{k}^{\dagger} c_{k}, \varepsilon_{\alpha}$ is given by the time-averaged band dispersion $\left\langle\left\langle\epsilon_{k}\right\rangle\right\rangle \equiv-1 / \mathscr{T} \int_{0}^{\mathscr{T}} d t \epsilon_{k}(t)$, and the Floquet wave function is $\Psi_{k}(t)=e^{-i\left\langle\left\langle\epsilon_{k}\right\rangle\right\rangle t} u_{k}(t)$ with $u_{k}(t)=$ $e^{-i \int_{0}^{t} d t\left[\epsilon_{k}(\bar{t})-\left\langle\left\langle\epsilon_{k}\right\rangle\right\rangle\right]}$ (Tsuji, Oka, and Aoki, 2008). Effectively, the band dispersion in the periodically driven system is renormalized from the original $\varepsilon_{k}$ to the time-averaged $\left\langle\left\langle\varepsilon_{\boldsymbol{k}}\right\rangle\right\rangle$, and the renormalized band splits into replicas with a spacing $\Omega$.

In practice, it is convenient to use the inverse $\boldsymbol{G}_{0}^{R-1}(\boldsymbol{k}, \omega)$ rather than $\boldsymbol{G}_{0}^{R}(\boldsymbol{k}, \omega)$ when one solves the Dyson equation (56). Using Eq. (184) and the unitarity of $\boldsymbol{U}_{\boldsymbol{k}}$, one has $\boldsymbol{G}_{0}^{R-1}(\boldsymbol{k}, \omega)=\boldsymbol{U}_{k} \boldsymbol{Q}_{k}^{-1}(\omega) \boldsymbol{U}_{k}^{\dagger}$, which reads

$$
\left(\boldsymbol{G}_{0}^{R-1}\right)_{m n}(\boldsymbol{k}, \omega)=(\omega+n \Omega+\mu+i \eta) \delta_{m n}-\left(\boldsymbol{\epsilon}_{\boldsymbol{k}}\right)_{m n} .
$$

Here $\boldsymbol{\epsilon}_{\boldsymbol{k}}$ is the Floquet matrix defined by

$$
\left(\boldsymbol{\epsilon}_{\boldsymbol{k}}\right)_{m n}=\frac{1}{\mathscr{T}} \int_{0}^{\mathscr{T}} d t e^{i(m-n) \Omega t} \epsilon_{\boldsymbol{k}}(t)
$$

The Keldysh component of the noninteracting Green's function is not uniquely determined by Eq. (182). One way to state this fact is to say that $\left(\boldsymbol{G}_{0}^{-1}\right)_{m n}^{K}(\boldsymbol{k}, \omega) \equiv-\left(\boldsymbol{G}_{0}^{R-1} \boldsymbol{G}_{0}^{K} \boldsymbol{G}_{0}^{A-1}\right)_{m n}(\boldsymbol{k}, \omega)=$ $2 i \eta F\left(\epsilon_{k}-\mu\right) \delta_{m n}$ is proportional to the infinitesimal $\eta$ and an arbitrary distribution function. The latter is usually fixed by the equilibrium distribution $F(\omega)=\tanh (\beta \omega / 2)$ [Eq. (29b)] for fermions. However, any other nonzero term in the Keldysh self-energy (e.g., a bath self-energy $\Sigma_{\text {bath }}^{K}$ ) dominates $\left(\boldsymbol{G}_{0}^{-1}\right)^{K}$ and thus completely determines the steady-state distribution.

\section{b. Simple example}

To see how the Floquet Green's function technique works, we consider a one-dimensional electric-field-driven tight-binding model (Han, 2013) coupled to free-fermion baths (Sec. II.A.2.b). In the temporal gauge, the Hamiltonian is

$$
H_{\mathrm{s}}(t)=-2 \gamma \sum_{k} \cos [k-A(t)] c_{k}^{\dagger} c_{k},
$$

where the dc electric field is introduced as the Peierls phase $A(t)=-\Omega t$, with the Bloch-oscillation frequency $\Omega=e E a$. Although $A(t)$ is not periodic in time, the Hamiltonian (189) has the periodicity with period $\mathscr{T}=2 \pi / \Omega$, and we can apply the Floquet method to this dc-electric-field problem. In the present case, we have $\epsilon_{k}(t)=-2 \gamma \cos (k+\Omega t)$, which leads to $\left\langle\left\langle\epsilon_{k}\right\rangle\right\rangle=0$ and

$$
\left(\boldsymbol{U}_{k}\right)_{m n}=e^{-i(m-n) k-2 i(\gamma / \Omega) \sin k} \mathcal{J}_{n-m}\left(\frac{2 \gamma}{\Omega}\right),
$$

where $\mathcal{J}_{n}$ is the $n$th order Bessel function. The Floquet Green's function is derived from the Dyson equation (56):

$$
\left(\begin{array}{cc}
\boldsymbol{G}^{R} & \boldsymbol{G}^{K} \\
O & \boldsymbol{G}^{A}
\end{array}\right)^{-1}=\left(\begin{array}{cc}
\boldsymbol{G}_{0}^{R-1} & \left(\boldsymbol{G}_{0}^{-1}\right)^{K} \\
O & \boldsymbol{G}_{0}^{A-1}
\end{array}\right)-\left(\begin{array}{cc}
\boldsymbol{\Sigma}_{\text {bath }}^{R} & \boldsymbol{\Sigma}_{\text {bath }}^{K} \\
O & \boldsymbol{\Sigma}_{\text {bath }}^{A}
\end{array}\right)
$$

where

$$
\left(\begin{array}{cc}
\boldsymbol{\Sigma}_{\text {bath }}^{R}(\omega) & \boldsymbol{\Sigma}_{\text {bath }}^{K}(\omega) \\
O & \boldsymbol{\Sigma}_{\text {bath }}^{A}(\omega)
\end{array}\right)=\left(\begin{array}{cc}
-i \Gamma \mathbb{1} & -2 i \Gamma \boldsymbol{F}(\omega) \\
O & i \Gamma \mathbb{1}
\end{array}\right)
$$

is the Floquet representation of the bath self-energy (59), with

$$
\boldsymbol{F}_{m n}(\omega) \equiv \tanh \left(\frac{\omega+n \Omega}{2 T}\right) \delta_{m n}
$$

the Floquet representation of the Fermi distribution function for fermions with the bath temperature $T$. With the decomposition (184), we have the retarded Floquet Green's function $\boldsymbol{G}^{R}=\boldsymbol{U}_{k} \cdot\left[\boldsymbol{Q}_{k}^{-1}(\omega)+i \Gamma \mathbb{1}\right]^{-1} \cdot \boldsymbol{U}_{k}^{\dagger}$, which reads

$$
\left(\boldsymbol{G}^{R}\right)_{m n}(k, \omega)=e^{-i(m-n) k} \sum_{l} \frac{\mathcal{J}_{l-m}(2 \gamma / \Omega) \mathcal{J}_{n-l}(2 \gamma / \Omega)}{\omega+l \Omega+i \Gamma} .
$$

The lesser Floquet Green's function is given by $\boldsymbol{G}^{<}=2 i \Gamma \boldsymbol{G}^{R}$. $\boldsymbol{f} \cdot \boldsymbol{G}^{A}$ with the Fermi distribution function $\boldsymbol{f}_{m n}=f(\omega+n \Omega) \delta_{m n}$. Using this, we obtain the momentum distribution function 
$n(\boldsymbol{k}, t)=-i \int_{-\Omega / 2}^{\Omega / 2}(d \omega / 2 \pi) \sum_{m n} e^{-i(m-n) \Omega t} \boldsymbol{G}_{m n}^{<}(\boldsymbol{k}, \omega)$, which can be evaluated analytically for temperature $T=0$ (Han, 2013):

$$
\begin{aligned}
n(k, t)= & \frac{\Gamma}{\pi} \sum_{m, n} e^{i(m-n)(k+\Omega t)} \frac{\mathcal{J}_{m}(2 \gamma / \Omega) \mathcal{J}_{n}(2 \gamma / \Omega)}{(n-m) \Omega+2 i \Gamma} \\
& \times \ln \left(\frac{m \Omega-i \Gamma}{n \Omega+i \Gamma}\right) .
\end{aligned}
$$

The function $n(k, t)$ defined in this way is not gauge invariant. To obtain a physical observable, we must evaluate it at a comoving wave vector $\tilde{k}=k-\Omega t$ (see Sec. II.B.5). Hence the gaugeinvariant momentum distribution function $\tilde{n}(k, t) \equiv n(\tilde{k}, t)=$ $n(k-\Omega t, t)$ is time independent. In the weak-field limit $(\Omega \ll \gamma, \Gamma)$, we have

$$
n(\tilde{k})=\frac{1}{2}+\frac{1}{\pi} \arctan \left(\frac{2 \gamma \cos (\tilde{k}-\delta k)}{\Gamma}\right)+O\left(\Omega^{2}\right)
$$

with the momentum shift

$$
\delta k=\frac{\Omega}{\Gamma}\left[1+\left(\frac{2 \gamma \cos \tilde{k}}{\Gamma}\right)^{2}\right]^{-1}
$$

At zero field,

$$
n(k)=\int d \omega \frac{\Gamma / \pi}{\left(\omega-\epsilon_{k}\right)^{2}+\Gamma^{2}} f(\omega)=\frac{1}{2}+\frac{1}{\pi} \arctan \left(\frac{-\epsilon_{k}}{\Gamma}\right) .
$$

When the field is turned on, the momentum distribution shifts in the field direction by $\delta k \sim \Omega / \Gamma$ near the Fermi surface, which is expected from Boltzmann's semiclassical transport theory with the relaxation time approximation $\left(\tau \sim \Gamma^{-1}\right)$. The current is obtained from

$$
j=\int \frac{d k}{2 \pi} v(k-A(t)) n(k, t)=\int \frac{d k}{2 \pi} v(k) \tilde{n}(k)
$$

with the group velocity $v(k)=\partial \epsilon(k) / \partial k$. It is time independent (no Bloch oscillations), and consistent with the linear-response result in the weak-field limit

$$
j=\frac{2 \gamma^{2}}{\pi \Gamma \sqrt{4 \gamma^{2}+\Gamma^{2}}} \Omega+O\left(\Omega^{2}\right) .
$$

When $\Gamma \ll \gamma$, it reproduces the Drude formula

$$
j \sim \frac{\gamma \Omega}{\pi \Gamma} \propto \frac{E \tau}{m^{*}}
$$

with $\gamma \sim 1 / m^{*}$ ( $m^{*}$ : effective mass) and $\Gamma \sim 1 / \tau(\tau$ : relaxation time). This shows that although the free-fermion bath model (Sec. II.A.2.b) is somewhat artificial in the sense that it only includes single-particle processes, it correctly reproduces the conventional semiclassical transport picture without momentum scattering, so that it serves as a minimal model for dissipation mechanisms.

\section{Floquet dynamical mean-field theory}

a. General formalism

In this section, we describe the application of Floquet theory to nonequilibrium DMFT, to study periodically driven states of strongly correlated systems. The original idea goes back to the pioneering work of Schmidt and Monien (2002). The formalism was further developed by Freericks and Joura (2008), Joura, Freericks, and Pruschke (2008), and Tsuji, Oka, and Aoki (2008, 2009). In the Floquet DMFT formalism, one considers a dissipative system continuously driven by a timeperiodic perturbation. It is assumed that a time-periodic NESS exists in the long-time limit, after all memory on the initial condition has been wiped out by the dissipation. [These assumptions have been numerically tested for the driven Hubbard model coupled to a heat bath by Amaricci et al. (2012).] Floquet DMFT can directly access this time-periodic NESS, without computing the full time evolution from the initial state, by mapping the time-periodic NESS of the lattice model to the corresponding time-periodic NESS of an effective single-site impurity model.

As an example, consider the Hubbard model, for which the action $S_{\mathrm{imp}}^{\mathrm{NESS}}$ of the effective impurity problem is the same as Eq. (75), but on the Keldysh contour $\mathcal{C}_{K}$ (Fig. 6) instead of the L-shaped contour, and with a hybridization function $\Delta_{\sigma}\left(t, t^{\prime}\right)$, that has the time periodicity $\Delta_{\sigma}\left(t+\mathscr{T}, t^{\prime}+\mathscr{T}\right)=\Delta_{\sigma}\left(t, t^{\prime}\right)$. Because of the periodicity in time, the impurity Green's function defined by

$$
G_{\sigma}^{\mathrm{imp}}\left(t, t^{\prime}\right)=-i\left\langle\mathcal{T}_{C_{K}} c_{\sigma}(t) c_{\sigma}^{\dagger}\left(t^{\prime}\right)\right\rangle_{S_{\mathrm{imp}}^{\mathrm{NESS}}}
$$

also satisfies $G_{\sigma}^{\mathrm{imp}}\left(t+\mathscr{T}, t^{\prime}+\mathscr{T}\right)=G_{\sigma}^{\mathrm{imp}}\left(t, t^{\prime}\right)$. As described in Sec. II.D.2, the time-periodic Green's function can thus be represented by a Floquet Green's function $\boldsymbol{G}_{\sigma}^{\text {imp }}(\omega)$ in frequency space. Each Green's function or self-energy in the Floquet representation then has a supermatrix structure of the Larkin-Ovchinnikov form

$$
\boldsymbol{G}=\left(\begin{array}{cc}
\boldsymbol{G}^{R} & \boldsymbol{G}^{K} \\
O & \boldsymbol{G}^{A}
\end{array}\right)
$$

The mapping from the lattice to the impurity model is defined such that the local part of the lattice Floquet Green's function is reproduced by the impurity Floquet Green's function

$$
\boldsymbol{G}_{\sigma}^{\mathrm{imp}}(\omega)=\sum_{\boldsymbol{k}} \boldsymbol{G}_{\sigma}^{\mathrm{lat}}(\boldsymbol{k}, \omega),
$$

and one makes the approximation that the lattice Floquet selfenergy $\boldsymbol{\Sigma}_{\sigma}^{\text {lat }}$ is local in space (or independent of $\boldsymbol{k}$ ) and can be identified with the Floquet self-energy of the impurity

$$
\boldsymbol{\Sigma}_{\sigma}^{\mathrm{lat}}(\boldsymbol{k}, \omega)=\boldsymbol{\Sigma}_{\sigma}^{\mathrm{imp}}(\omega)
$$

The lattice Floquet Green's function satisfies the Dyson equation for the lattice model

$$
\boldsymbol{G}_{\sigma}^{\text {lat }}(\boldsymbol{k}, \omega)=\left[\boldsymbol{G}_{0 \sigma}^{-1}(\boldsymbol{k}, \omega)-\boldsymbol{\Sigma}^{\text {bath }}(\omega)-\boldsymbol{\Sigma}_{\sigma}^{\text {lat }}(\boldsymbol{k}, \omega)\right]^{-1},
$$

where $\boldsymbol{G}_{0 \sigma}(\boldsymbol{k}, \omega)$ is the noninteracting Floquet Green's function and $\boldsymbol{\Sigma}^{\text {bath }}(\omega)$ is a dissipation term coming from the 
coupling to the external heat bath. Usually $\boldsymbol{\Sigma}^{\text {bath }}(\omega)$ is a local function, such as the Floquet representation (192) of Eq. (55) for the free-fermion bath. The impurity Floquet Green's function satisfies the Dyson equation for the impurity model

$$
\boldsymbol{G}_{\sigma}^{\mathrm{imp}}(\omega)=\left[\boldsymbol{\omega}+\mu \mathbb{1}-\boldsymbol{\Delta}_{\sigma}(\omega)-\boldsymbol{\Sigma}_{\sigma}^{\mathrm{imp}}(\omega)\right]^{-1},
$$

with $\omega_{m n}=(\omega+n \Omega) \delta_{m n}$. The self-consistency condition of the Floquet DMFT consists of Eqs. (202), (203), (204), and (205) in combination with the solution of the effective impurity problem (200).

\section{b. Impurity solver}

In practical implementations of the Floquet DMFT, one has to solve the time-periodic nonequilibrium impurity problem (200). Since the length of the real-time axis is infinite by construction, the available impurity solvers (Sec. II.C) are limited. For example, quantum Monte Carlo techniques cannot be used due to the dynamical sign problem. Joura, Freericks, and Pruschke (2008) applied the numerical renormalization group technique (Bulla, Costi, and Pruschke, 2008) to calculate the density of states for the NESS of the driven Hubbard model based on the approximation of using the thermal density matrix. Tsuji, Oka, and Aoki (2009) studied the FalicovKimball model (Freericks and Zlatić, 2003) driven by an ac field with the Floquet DMFT, for which an exact solution for the impurity problem out of equilibrium can be used. Lubatsch and Kroha (2009) employed the iterated perturbation theory as an impurity solver to study the Hubbard model driven by an ac field, where the field was introduced to linear order by the $\boldsymbol{j} \cdot \boldsymbol{A}$ coupling. In general, one can directly apply diagrammatic techniques such as the weak-coupling (Sec. II.C.4) and strongcoupling (II.C.5) perturbation theories to the impurity problem. In the presence of a heat bath, numerical simulations usually become stable, even for nonconserving approximations.

\section{E. Extensions of DMFT and alternative approaches}

Single-site dynamical mean field theory provides a qualitatively correct description of high-dimensional lattice models. Since nonequilibrium applications often involve highly excited systems, and nonlocal correlations tend to become less relevant in equilibrium models at high temperature, one might expect that the local self-energy approximation of single-site DMFT is even better in the nonequilibrium context than it is in equilibrium. On the other hand, as mentioned in Sec. II.B.2, even small perturbations to the Hamiltonian can have a pronounced influence on the long-time behavior of a system out of equilibrium. It is thus not a priori obvious how severely the single-site DMFT approximation affects the time evolution. To study the nonequilibrium properties of models with reduced dimensionality, it is important to develop methods which take spatial correlations into account. Extensions of the DMFT formalism are also needed to study inhomogeneous systems, such as heterostructures or cold atoms in a trapping potential.

\section{Cluster perturbation theory}

A simple and computationally cheap method which allows one to treat short-range correlations explicitly is the cluster perturbation theory (CPT) (Gros and Valentí, 1993; Sénéchal, Perez, and Pioro-Ladrière, 2000). This method has recently been adapted to nonequilibrium systems (Balzer and Potthoff, 2011; Knap, von der Linden, and Arrigoni, 2011). The idea is to decompose the system into small clusters, whose dynamics can be computed exactly, for example, using exact diagonalization or Krylov-space methods (Balzer, Gdaniec, and Potthoff, 2012), and to treat the intercluster hopping as a perturbation. An example of such a decomposition is shown in Fig. 14, for a system consisting of several layers with local interactions $U$, sandwiched between two noninteracting leads, where we chose clusters of length $L_{x}=N$ and width $L_{y}=2$ (dashed lines). If we denote the cluster part (local terms and intracluster hoppings) of the Hamiltonian by $h$ and the intercluster hopping terms by $T$, we obtain the decomposition

$$
H=h+T \text {. }
$$

We denote the Green's function and self-energy corresponding to $H$ by $G$ and $\Sigma$, and those corresponding to the unperturbed Hamiltonian $h$ by $g$ and $\Sigma_{h}$. When these functions are viewed as matrices in intracluster orbital and spin indices, the Dyson equation becomes

$$
G=g+g(T+\delta \Sigma) G
$$

with $\delta \Sigma=\Sigma-\Sigma_{h}$. In a nonequilibrium calculation, $G$ and $g$ are Keldysh Green's functions, and the product is some convolution (depending on whether one works within the Kadanoff-Baym formalism, the Floquet formalism, or the Keldysh formalism for steady states). The CPT approximation neglects $\delta \Sigma$, i.e., we compute an approximate lattice Green's function $G_{\mathrm{CPT}}$ using the Dyson equation

$$
G_{\mathrm{CPT}}=g+g T G_{\mathrm{CPT}} .
$$

Only $\Sigma_{h}$ is taken into account (in the exact calculation of $g$ ). In a setup with leads like in Fig. 14, we can restrict the description to the correlated region, and denote the full Green's function and the Green's function of the cluster part by $G_{c}$ and $g_{c}$, respectively. The effect of the noninteracting leads is to add a lead self-energy $\Sigma_{\text {leads }}=\Sigma_{L}+\Sigma_{R}$, with $\Sigma_{L}=$ $T_{L c} g_{L} T_{c L}$ [cf. Eq. (55)] and similarly for $\Sigma_{R}$ (here $g_{\alpha}$ denotes the Green's function for lead $\alpha$ and $T_{c \alpha}$ the hopping between

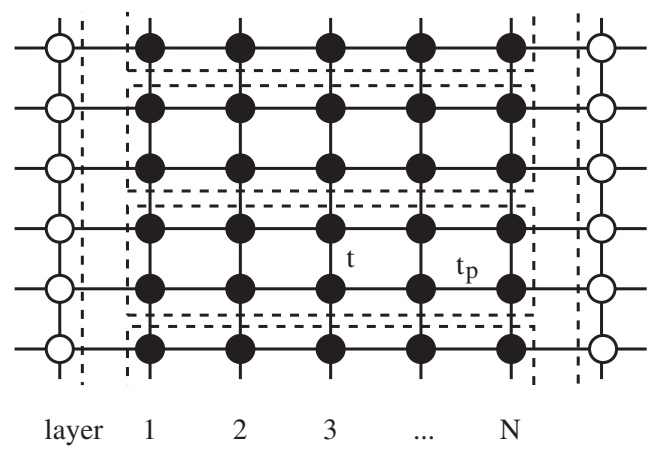

FIG. 14. Illustration of a system consisting of $N$ correlated layers (full dots) between noninteracting leads (open dots). The intralayer hopping $t$, interlayer hopping $t_{p}$, interaction $U$, and chemical potential $\mu$ can be layer and time dependent. 
the correlated region and lead $\alpha$ ). Hence, we have to solve the Dyson equation

$$
G_{c}=g_{c}+g_{c}\left(T_{c}+\Sigma_{\text {leads }}\right) G_{c}
$$

for known $g_{c}$, which can be done using the techniques described in Sec. II.B.

Balzer and Potthoff (2011) showed by comparison to the exact solution that nonequilibrium CPT correctly reproduces the short-time dynamics in small lattice systems. The advantages of this approach are that it respects causality, treats correlations exactly within the cluster, and is moderate in terms of computational cost. Both the noninteracting limit $(U=0)$ and the isolated cluster limit $T=0$ are recovered exactly. Furthermore, although the dynamics of the exactly solved small cluster shows severe finite-size artifacts, such as persistent beating oscillations after a perturbation, the Dyson equation (208) can restore the relaxation that is characteristic for an infinite-size system Jung et al. (2012). This fact is illustrated in Fig. 15.

Nevertheless, a main limitation of the approach is the limited feedback to the exactly solved subsystem. A possibility to partly resolve this issue is to perform a self-consistent resummation of certain classes of correction terms to the isolated cluster (or single-site) self-energy (Mikelsons, Freericks, and Krishnamurthy, 2012), in the spirit of a linked cluster expansion around the atomic limit (Metzner, 1991). As an alternative way to improve the method by including a feedback to the reference system, Knap, von der Linden, and Arrigoni (2011) proposed a nonequilibrium generalization of the variational cluster approach (VCA) (Potthoff, Aichhorn, and Dahnken, 2003) for nonequilibrium steady states. This generalization exploits the fact that the decomposition (206) of the Hamiltonian into a cluster contribution $h$ and a perturbation

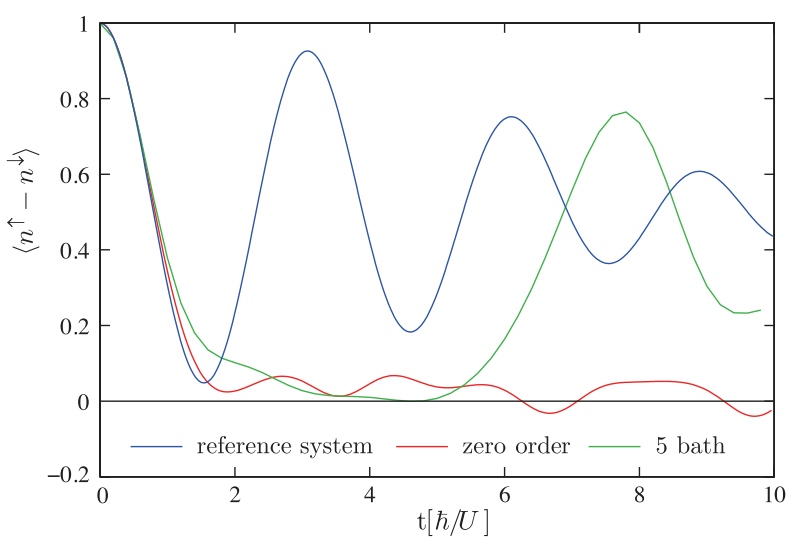

FIG. 15 (color online). Time evolution of the magnetization in a semi-infinite chain with interaction $U=1$ on the first (impurity) site. The hopping between the noninteracting bath sites is $t=1$ and the initial state is the impurity, occupied with a spin-up electron, decoupled from the bath. The time evolution is triggered by the sudden switch-on of the hopping between the impurity and the bath. The curves show the time evolution of the spin polarization in the impurity, computed in a finite chain with five bath sites, the CPT result for a reference cluster $C^{\prime}$ that consists of only the impurity and one bath site ("zeroth order"), and for the isolated cluster $C^{\prime}$ ("reference system"). From Jung et al., 2012.
$T$ is not unique. We can add arbitrary single-particle terms $\delta h$ to $h$, provided that we subtract them from $T$, that is, we can write $H=\tilde{h}+\tilde{T}$ with $\tilde{h}=h+\delta h$ and $\tilde{T}=T-\delta h$. By optimizing the parameters of these additional single-particle terms one can hope to achieve a better description of the system. The main question is how the optimization should be done in practice. For nonequilibrium steady states, Knap, von der Linden, and Arrigoni (2011) proposed the following: denote the variational parameters (intracluster hoppings and on-site energies) by $p$, and the operators coupled to these parameters by $O_{p}$ $\left(O_{p}=d \delta h / d p\right)$. The self-consistency condition which fixes the parameter $p$ demands that the expectation value of the operators $O_{p}$ are the same in the unperturbed and in the perturbed state. With an infinite number of variational parameters, corresponding to an infinite number of bath sites attached to the cluster, the above procedure allows one to optimize the system in such a way that the cluster Green's function $g_{c}$ becomes identical to the cluster projection of the full Green's function $G_{c}$. This is precisely the self-consistency condition which determines the bath parameters in the cluster extension of DMFT (cellular DMFT). The extension of the corresponding variational principle to time-evolving systems, rather than steady states, has been proposed recently (Hofmann et al., 2013), but has not yet been implemented numerically.

\section{Cluster extension of nonequilibrium DMFT}

In cluster extensions of DMFT (Maier et al., 2005), one maps the lattice model to a multisite cluster embedded in a dynamical mean-field bath, which is self-consistently determined. When the number of cluster sites $N_{c}$ is 1 , the formalism reduces to the original DMFT. By increasing $N_{c}$, one can systematically introduce the momentum dependence of the self-energy, which has been neglected in DMFT. This allows one to address the role and importance of spatially nonlocal correlations in the nonequilibrium dynamics of correlated systems, especially in low dimensions.

The mapping to the cluster model is not unique unlike the single-site DMFT. There are two well-established approaches to construct a cluster extension of DMFT, namely, the cellular DMFT (Lichtenstein and Katsnelson, 2000; Kotliar et al., 2001) and the dynamical cluster approximation (DCA) (Hettler et al., 1998, 2000). They differ in the way the effective cluster problem is constructed. Since the nonequilibrium generalization of both methods is straightforward, we briefly review here, as an example, the nonequilibrium DCA for the Hubbard model (Tsuji et al., 2013), which enforces translational symmetries. The action of the effective cluster problem for DCA is a functional of a hybridization function $\Delta_{\sigma}\left(\boldsymbol{R} ; t, t^{\prime}\right)$ :

$$
\begin{aligned}
S_{\text {clust }}[\Delta]= & \sum_{\boldsymbol{R} \boldsymbol{R}^{\prime} \sigma} \int_{C} d t\left(v_{\boldsymbol{R}, \boldsymbol{R}^{\prime}}-\mu \delta_{\boldsymbol{R}, \boldsymbol{R}^{\prime}}\right) d_{\boldsymbol{R} \sigma}^{\dagger}(t) d_{\boldsymbol{R}^{\prime} \sigma}(t) \\
& +\sum_{\boldsymbol{R} \boldsymbol{R}^{\prime} \sigma} \int_{C} d t \int_{C} d t^{\prime} d_{\boldsymbol{R} \sigma}^{\dagger}(t) \Delta_{\sigma}\left(\boldsymbol{R}-\boldsymbol{R}^{\prime} ; t, t^{\prime}\right) d_{\boldsymbol{R}^{\prime} \sigma}\left(t^{\prime}\right) \\
& +U \sum_{\boldsymbol{R}} \int_{C} d t \hat{n}_{\boldsymbol{R} \uparrow}(t) \hat{n}_{\boldsymbol{R} \downarrow}(t),
\end{aligned}
$$

where $\mathcal{C}$ is the L-shaped contour (Sec. II.A), and the cluster sites are labeled by $\boldsymbol{R}$ and $\boldsymbol{R}^{\prime}$ with a hopping amplitude $v_{\boldsymbol{R}, \boldsymbol{R}^{\prime}}$. By 
solving the cluster problem, one obtains the cluster Green's function $G_{\sigma}^{\text {clust }}\left(\boldsymbol{R} ; t, t^{\prime}\right)=-i\left\langle\mathcal{T}_{C} d_{\boldsymbol{R} \sigma}(t) d_{\boldsymbol{0} \sigma}^{\dagger}\left(t^{\prime}\right)\right\rangle_{S_{\text {clust }}}$. After Fourier transformation, it is represented in momentum space as $G_{\sigma}^{\text {clust }}\left(\boldsymbol{K} ; t, t^{\prime}\right)$, where $\boldsymbol{K}$ is a reciprocal vector of $\boldsymbol{R}$. The Brillouin zone is then divided into $N_{c}$ patches, centered at the wave vectors $\boldsymbol{K}$. In the lattice problem, an arbitrary wave vector $\boldsymbol{k}$ is represented by $\boldsymbol{k}=\boldsymbol{K}+\tilde{\boldsymbol{k}}$, where $\tilde{\boldsymbol{k}}$ is a wave vector of the superlattice defined by the clusters. The cluster problem is constructed such that the cluster Green's function corresponds to the lattice Green's function averaged over each patch, i.e.,

$$
G_{\sigma}^{\text {clust }}\left(\boldsymbol{K} ; t, t^{\prime}\right)=\frac{N_{c}}{N} \sum_{\tilde{\boldsymbol{k}}} G_{\sigma}^{\mathrm{lat}}\left(\boldsymbol{K}+\tilde{\boldsymbol{k}} ; t, t^{\prime}\right),
$$

with $N$ the total number of $k$ points in the Brillouin zone. The approximation of the method is to identify the lattice self-energy with the cluster self-energy

$$
\Sigma_{\sigma}^{\text {lat }}\left(\boldsymbol{K}+\tilde{\boldsymbol{k}} ; t, t^{\prime}\right)=\Sigma_{\sigma}^{\mathrm{clust}}\left(\boldsymbol{K} ; t, t^{\prime}\right)
$$

i.e., the $\tilde{\boldsymbol{k}}$ dependence of $\Sigma_{\sigma}^{\mathrm{lat}}\left(\boldsymbol{K}+\tilde{\boldsymbol{k}} ; t, t^{\prime}\right)$ is neglected. With this, the equations for the Green's function and self-energy are closed, and one can obtain the self-consistency condition using the lattice Dyson equation

$$
\left(i \partial_{t}+\mu-\epsilon_{\boldsymbol{k}}\right) G_{\sigma}^{\mathrm{lat}}(\boldsymbol{k})-\Sigma_{\sigma}^{\mathrm{lat}}(\boldsymbol{k}) * G_{\sigma}^{\mathrm{lat}}(\boldsymbol{k})=\delta_{C}\left(t, t^{\prime}\right),
$$

and the cluster Dyson equation

$$
\begin{gathered}
\left(i \partial_{t}+\mu-\bar{\epsilon}_{\boldsymbol{K}}\right) G_{\sigma}^{\mathrm{clust}}(\boldsymbol{K})-\Delta_{\sigma}(\boldsymbol{K}) * G_{\sigma}^{\mathrm{clust}}(\boldsymbol{K}) \\
-\Sigma_{\sigma}^{\mathrm{clust}}(\boldsymbol{K}) * G_{\sigma}^{\mathrm{clust}}(\boldsymbol{K})=\delta_{C}\left(t, t^{\prime}\right)
\end{gathered}
$$

with $\bar{\epsilon}_{\boldsymbol{K}}=\left(N_{c} / N\right) \sum_{\tilde{\boldsymbol{k}}} \epsilon_{\boldsymbol{K}+\tilde{\boldsymbol{k}}}$.

An open issue is how to solve the nonequilibrium cluster problem. The impurity solvers used for the nonequilibrium DMFT (Sec. II.C) are in principle applicable to cluster problems. The CTQMC is a numerically exact method, but its applicability for cluster problems is very limited since the dynamical sign problem is expected to become even more severe than for single-site impurity problems. In the weakcoupling regime, the weak-coupling perturbation theory is most promising, since it is easily generalized by assigning cluster-site labels $\boldsymbol{R}$ to each interaction vertex, and is computationally feasible for large clusters (Tsuji et al., 2013). In the strong-coupling regime, the NCA-type expansion can be extended straightforwardly. However, the bare level propagators $g$ then have a $D \times D$ orbital matrix structure, where $D$ is the dimension of the Hilbert space of the cluster (respecting symmetries). The memory requirements for $g$ are of the order of $\sim D^{2} N_{t}^{2}$ complex numbers (where $N_{t}$ is the number of time steps), such that NCA in nonequilibrium is restricted to rather small clusters (e.g., $D_{\max }=12$ for $N_{c}=4$, using spatial symmetries).

\section{Dual fermions}

A systematic diagrammatic extension of single-site DMFT, which is also related to the VCA, is the dual-fermion method (Rubtsov, Katsnelson, and Lichtenstein, 2008). The idea here is to represent the lattice model as a collection of impurity models (with the same local interactions as in the lattice), plus quadratic terms, and to formulate a systematic expansion in the quadratic terms which provide the coupling between the impurities. The extension of this method to nonequilibrium systems has been detailed by Jung et al. (2012).

At present, the dual-fermion method has been implemented and tested for the nonequilibrium dynamics of impurity systems, within the framework of superperturbation theory (Hafermann et al., 2009). In this scheme, one chooses a reference impurity model with a small enough Hilbert space that the impurity Green's functions and vertex functions can be computed exactly. As in the VCA case, the parameters of the impurity model may be treated as parameters which can be optimized to achieve a better description within a low-order approximation. We briefly summarize this formalism, following Jung et al. (2012). The action of the impurity system is

$$
\begin{aligned}
S\left[d^{\dagger}, d\right] & =-i \int d t d t^{\prime} \sum_{a, b} d_{a}^{\dagger}(t) \Delta_{a b}\left(t, t^{\prime}\right) d_{b}\left(t^{\prime}\right)+S_{\mathrm{loc}} \\
& \equiv-i d_{1}^{\dagger} \Delta_{12} d_{2}+S_{\mathrm{loc}}
\end{aligned}
$$

where $a$ denotes spin and orbital indices and $\Delta$ is the hybridization function, which is related to the bath Green's function $\mathcal{G}_{0}$ by Eq. (128). In the second line, we introduce subscripts which represent combined spin, orbital and time indices, and assume a summation (contour integration) over repeated indices. The idea is to introduce a reference impurity system with a finite number of bath levels, corresponding to the hybridization function $\tilde{\Delta}$

$$
\tilde{S}\left[d^{\dagger}, d\right]=-i d_{1}^{\dagger} \tilde{\Delta}_{12} d_{2}+S_{\mathrm{loc}},
$$

such that the original action is given by the sum of the action of the reference system and a quadratic correction term $i d_{1}^{\dagger}\left(\tilde{\Delta}_{12}-\Delta_{12}\right) d_{2}$. In order to formulate the perturbation expansion around the reference system, dual fermions $f^{\dagger}$ and $f$ are introduced via a Gaussian integral with coupling term $i f^{\dagger}(t) g^{-1}\left(t, t^{\prime}\right) d\left(t^{\prime}\right)$, and $g$ the Green's function of the reference problem, leading to the action

$$
\begin{aligned}
S\left[d^{\dagger}, d, f^{\dagger}, f\right]= & \tilde{S}\left[d^{\dagger}, d\right]+S^{c}\left[d^{\dagger}, d, f^{\dagger}, f\right] \\
& +i f_{1}^{\dagger}\left[g^{-1}(\tilde{\Delta}-\Delta)^{-1} g^{-1}\right]_{12} f_{2},
\end{aligned}
$$

with $S^{c}\left[d^{\dagger}, d, f^{\dagger}, f\right]=-i f_{1}^{\dagger} g_{12}^{-1} d_{2}-i d_{1}^{\dagger} g_{12}^{-1} f_{2}$. The last step is to integrate out the original $d$ fermions, which leads to the dual action

$$
S^{d}\left[f^{\dagger}, f\right]=-i f_{1}^{\dagger} \Delta_{12}^{d} f_{2}-i \frac{1}{4} \gamma_{1234}^{4} f_{1}^{\dagger} f_{2} f_{3}^{\dagger} f_{4}+\cdots .
$$

Here $\gamma^{(4)}$ is the two-particle vertex of the reference system

$$
\gamma_{1234}^{4}=g_{11^{\prime}}^{-1} g_{33^{\prime}}^{-1}\left(\chi_{1^{\prime} 2^{\prime} 3^{\prime} 4^{\prime}}-\chi_{1^{\prime} 2^{\prime} 3^{\prime} 4^{\prime}}^{0}\right) g_{2^{\prime} 2}^{-1} g_{4^{\prime} 4}^{-1},
$$

with $\chi$ and $\chi^{0}$ the full and disconnected two-particle Green's function of the reference problem. The bare dual Green's function is defined as $G_{0}^{d}=-g\left[g+(\tilde{\Delta}-\Delta)^{-1}\right]^{-1} g$. The partition function of the dual theory may now be expanded in powers of $\gamma$, which leads to the dual self-energy 


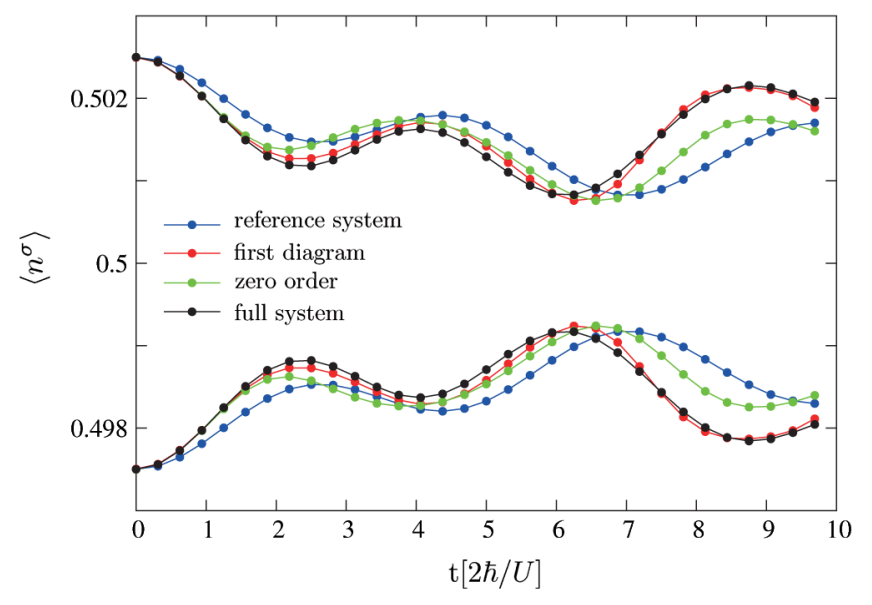

FIG. 16 (color online). Time evolution of the impurity occupation in a two-site system (one impurity with interaction $U=2$, one noninteracting bath site, hopping $v=0.5$ switched on at $t=0)$. The exact solution is compared to various approximations within the dual-fermion method (see text). From Jung et al., 2012.

$$
\Sigma_{12}^{d}=-i \gamma_{1234}^{4}\left(G_{0}^{d}\right)_{43}+\cdots
$$

Because of the complexity of calculating and storing higherorder vertices, practical implementations of the dual-fermion scheme will likely be restricted to the lowest-order diagram.

Once an approximate solution for the dual Green's function $G^{d}$ has been obtained, the Green's function $G$ of the original impurity problem is calculated using

$$
G=(\tilde{\Delta}-\Delta)^{-1}+[g(\tilde{\Delta}-\Delta)]^{-1} G^{d}[(\tilde{\Delta}-\Delta) g]^{-1} .
$$

A test calculation which illustrates the potential of the dualfermion method is shown in Fig. 16. The impurity system itself is only a two-site problem, consisting of one impurity (interaction $U=2$ ) coupled to one bath site. A hopping $v=$ 0.5 is switched on at time $t=0$. The reference system is the same model with a different hopping $\left(v^{\prime}=0.4\right.$, also switched on at $t=0$ ), and an expansion is performed in the difference $v-v^{\prime}$. Taking into account only the first dual diagram (220) leads to a considerable improvement over the zeroth order ( $\Sigma^{d}=0$, which is equivalent to $\mathrm{VCA}$ ), or the solution of the reference problem alone.

\section{Inhomogeneous DMFT}

An approximate treatment of inhomogeneous systems is possible with the "inhomogeneous" or "real-space" extension of DMFT (Potthoff and Nolting, 1999; Freericks, 2004), in which the self-energy is local, but site dependent. This technique can be adapted to nonequilibrium situations (Okamoto, 2007, 2008; Eckstein and Werner, 2013b) in order to describe, e.g., the nonlinear transport through correlated heterostructures, the trapping potential in cold-atom experiments, or time-dependent surface phenomena in condensed matter systems, such as the propagation of excitations from the surface of a sample into the bulk (André, Schiró, and Fabrizio, 2012). In the most general setup, the two space and two time arguments of the Green's function $G_{i j}\left(t, t^{\prime}\right)$ cannot be decoupled, neither by introducing momentum-dependent Green's functions $G_{k}\left(t, t^{\prime}\right)$ (as in homogeneous nonequilibrium DMFT) nor by using frequency-dependent Green's functions $G_{i j}(\omega)$ (as in inhomogeneous equilibrium DMFT). Inhomogeneous nonequilibrium DMFT simulations thus require a very large amount of memory in general. The problem turns out to be numerically tractable for a simpler layered geometry (Potthoff and Nolting, 1999; Freericks, 2004), in which the properties depend on the lattice position in one direction, but are homogeneous in the $d-1$ other dimensions.

To describe the approach, we consider the model illustrated in Fig. 14, consisting of $N$ correlated layers and connected to uncorrelated leads, with interlayer hopping $t_{p}$. [The equations can easily be generalized to a time- and layer-dependent interlayer hopping (Eckstein and Werner, 2013b).] After Fourier transformation within the layers ( $y$ direction), we have the following $N \times N$ matrix expression for the momentumdependent Green's function $G_{k}$ :

$\left(G_{k}^{-1}\right)_{m, n}=\left(i \partial_{t}+\mu-\epsilon_{k, m}-\Sigma_{m}\right) \delta_{m, n}-t_{p}\left(\delta_{m, n+1}+\delta_{m+1, n}\right)$,

where $\epsilon_{k, n}$ is the intralayer dispersion of layer $n$. (Time arguments $t, t^{\prime}$ on both sides of the equation are omitted for simplicity.) For the inhomogeneous DMFT calculation, we have to compute the local Green's functions $G_{n}=$ $-1 / N_{k} \sum_{k}\left(G_{k}\right)_{n, n}$ for the different layers, and hence we only need the diagonal elements $\left(G_{k}\right)_{n, n}$ of the momentumdependent Green's function. Because Eq. (222) is essentially the Dyson equation for a linear hopping chain, the diagonal elements $\left(G_{k}\right)_{n, n}$ can be obtained recursively, instead of treating the full $N \times N$ matrix problem:

$$
\left(G_{k}\right)_{n, n}^{-1}=i \partial_{t}+\mu-\epsilon_{k, n}-\Sigma_{n}-t_{p}^{2} G_{k, n-1}^{[n]}-t_{p}^{2} G_{k, n+1}^{[n]},
$$

where $\left(G_{k}^{[n]}\right)$ denotes the Green's function for a chain with site $n$ removed. The latter satisfy the Dyson equations

$$
\left(G_{k, n}^{[n \pm 1]}\right)^{-1}=i \partial_{t}+\mu-\epsilon_{k, n}-\Sigma_{n}-t_{p}^{2} G_{k, n \mp 1}^{[n]},
$$

for $n=1, \ldots, N$. Boundary conditions must be defined for $G_{k, 0}^{[1]}$ (left lead) and $G_{k, N+1}^{[N]}$ (right lead). (For a free surface, the hopping $t_{p}$ is set to 0 between the surface layer and the vacuum.) Once $G_{k, n-1}^{[n]}$ and $G_{k, n+1}^{[n]}$ for a given layer $n$ have been updated, one computes $\left(G_{k}\right)_{n, n}$ using Eq. (223), and determines the hybridization function $\Delta_{n}=\Delta_{n}\left[G_{n}\right]$ by solving the impurity Dyson equation

$$
G_{n}=\frac{1}{N_{k}} \sum_{k}\left(G_{k}\right)_{n, n} \equiv\left[i \partial_{t}+\mu-\Sigma_{n}-\Delta_{n}\right]^{-1}
$$

The hybridization function is the input for the impurity solver, which in turn yields an updated $G_{n}$ and $\Sigma_{n}$. A detailed description of the nonequilibrium implementation of inhomogeneous DMFT can be found in Eckstein and Werner (2013b). 


\section{APPLICATIONS}

In this section, we review nonequilibrium DMFT results for different types of nonequilibrium situations. On the one hand, for the theoretical investigation of pump-probe spectroscopy for solid-state systems, the effect of time-dependent electric fields on the electrons must be determined on the femtosecond time scale (Sec. III.A). On the other hand, a parameter in the Hamiltonian might be changed as a function of time, either abruptly (quench) (Sec. III.B.1) or gradually (ramp) (Sec. III.B.2). These changes are most readily realized in experiments on cold atomic gases in optical lattices, which allow precise control of the interaction and hopping parameters and can be very well isolated from the environment (Bloch, Dalibard, and Zwerger, 2008).

\section{A. Electric fields}

\section{Overview of field-induced phenomena}

Electron systems in strong electric fields raise a broad range of interesting issues, from linear and nonlinear transport to the fundamental question whether it is possible to control phase transitions by external fields. On the experimental side, the effect of an electric field in solids can be studied either by a nonlinear transport measurement or by studying the nonlinear optical response. For an insulator, one can roughly distinguish the regimes of nonlinear optics (ac response) and nonlinear transport (dc response) as a function of the field strength $F$ and the laser frequency $\Omega$ by the Keldysh line $F_{K} \sim \Omega / \xi$ ( $\xi$ is the correlation length that characterizes the length scale of an insulator) (see Fig. 17): Nonlinear transport is characterized by electron-hole production due to field-induced quantum tunneling across the gap, while in the regime of nonlinear optics one has generally multiphoton absorptions and emissions (Oka, 2012). In the following, we briefly describe various electric field-induced phenomena which are of interest in strongly correlated systems and can possibly be investigated using nonequlibrium DMFT.

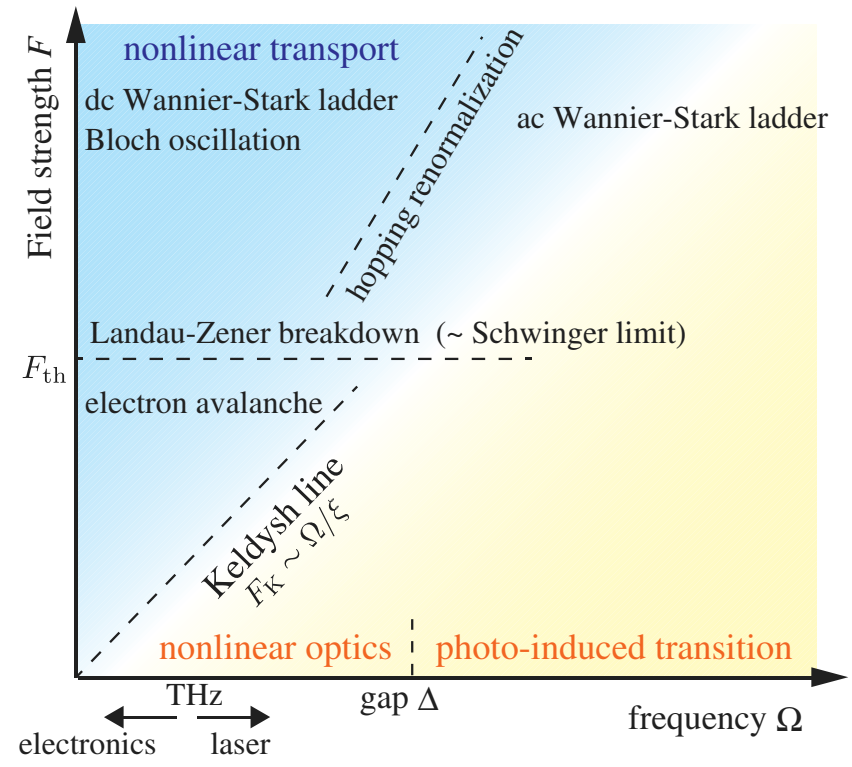

FIG. 17 (color online). Various regimes of electric field-induced phenomena plotted against the field strength $F$ and frequency $\Omega$.
Nonlinear transport: Many interesting phenomena have been reported on nonlinear transport properties in correlated electron systems, such as the colossal electroresistance, which corresponds to a large memory effect in the $I-V$ characteristics (Asamitsu et al., 1997; Oshima et al., 1999; Liu, Wu, and Ignatiev, 2000; Sawa et al., 2004), the thyristor effect, in which current oscillations emerge (Sawano et al., 2005), or a negative differential resistance, which is observed in many correlated insulators (Taguchi, Matsumoto, and Tokura, 2000; Inada et al., 2009; Mori et al., 2009).

Photo induced phase transitions: Nonequilibrium phase transitions can be realized by applying a laser with photon energy exceeding the energy gap of a given system (Nasu, 2004; Tokura, 2006; Yonemitsu and Nasu, 2008). Since nonequilibrium phase transitions can result in large responses, these phenomena are expected to lead to novel devices such as all optical memories.

Dielectric breakdown of insulators: When a dc electric field in an insulator exceeds a critical value, quantum tunneling causes pair creation of charge carriers. For a band insulator, this is known as the Landau-Zener breakdown, which corresponds to the Schwinger effect in nonlinear QED. Another possible mechanism for the dielectric breakdown is the electron avalanche effect: when the kinetic energy of thermal electrons accelerated in an electric field exceeds the paircreation energy, an exponential growth in the carrier density occurs. This was demonstrated in semiconductors irradiated with terahertz lasers (Hirori, Shinokita et al., 2011) as well as in a Mott insulator in dc fields (Guiot et al., 2013).

Nonlinear optical responses: In the context of correlated electron systems, a giant nonlinearity in the optical response has been reported in 1D Mott insulators (Kishida et al., 2000; Mizuno et al., 2000).

Bloch oscillations: Bloch oscillations result from coherent periodic motions of particles in a lattice system driven by strong electric fields. In strong electric fields, the electron wave function becomes localized in the direction of the field. This phenomenon is called Wannier-Stark localization, and results in a ladder structure in the energy spectrum. DMFT allows one to study the interplay of interactions and fieldinduced localization.

Hopping renormalization: The hopping parameter is renormalized in strong ac fields with a Bessel function; cf. Eq. (228). This can lead to a band flipping.

Many of the above-listed strong-field effects have recently been studied with nonequilibrium DMFT and in the following sections, we summarize some key results.

\section{2. dc electric fields}

a. Bloch oscillations

In the absence of electron scattering, a dc electric field applied to a metallic system will result in an undamped oscillating current, a phenomenon known as Bloch oscillations (Bloch, 1929; Zener, 1934). Because the period of the Bloch oscillations is typically much longer than the scattering time for experimentally accessible field strengths, these oscillations can hardly be observed in metals. On the other hand, they have been studied intensively in semiconductor heterostructures (Glück, Kolovsky, and Korsch, 2002). Also 


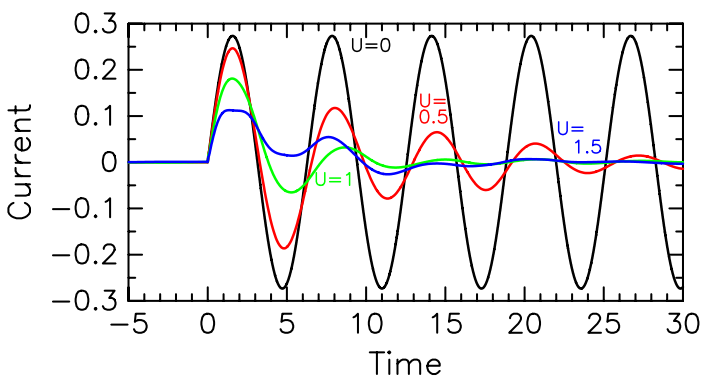

FIG. 18 (color online). Damped Bloch oscillations in the Falicov-Kimball model with a constant electric field $F=1$, and indicated values of the interaction $U$. From Freericks, Turkowski, and Zlatić, 2006.

cold-atom systems (Ben Dahan et al., 1996) are ideally suited to study this intrinsic nonequilibrium phenomenon, and its dependence on the underlying lattice structure (Tarruell et al., 2012).

In a noninteracting tight-binding model, the origin of the oscillations can be understood either as arising from a timedependent shift of the occupied momentum states in $\boldsymbol{k}$ space with reflections at the Brillouin zone boundary or alternatively as a localization of the wave packet in a linear potential gradient. An interesting theoretical question is what will happen to this oscillating current if electron-electron scattering is taken into account. In particular, one may wonder if and how a dc response is established at long times. A numerical investigation of Bloch oscillations in the half-filled FalicovKimball model (83) has been undertaken in the pioneering nonequilibrium DMFT papers by Freericks and collaborators (Freericks, Turkowski, and Zlatić, 2006; Turkowski and Freericks, 2007b; Freericks, 2008) (see also Tran, 2008). Figure 18 shows the (rescaled) current induced by a constant electric field $F=1$ (in a model with a Gaussian density of states, whose variance sets the unit of energy). This model has a Mott transition at $U=\sqrt{2}$, so the curve for $U=0.5$ corresponds to a moderately correlated metal, $U=1.0$ to a strongly correlated metal, while $U=1.5$ shows the current induced in a Mott insulating, but nearly critical system. One can clearly see the damping of the Bloch oscillations with increasing electronelectron scattering, which becomes quite incoherent as one goes across the metal-insulator transition point. Similar investigations in the metallic phase of the Hubbard model (using second-order weak-coupling perturbation theory and CTQMC as an impurity solver) show a sharp crossover between a dc regime, in which the current at long times is given by the linearresponse conductivity, and an ac regime, in which Bloch oscillations persist for all times until the system reaches an infinite-temperature state with zero current (Eckstein and Werner, 2011a).

The many-body density of states approaches a steady-state limit characterized by Wannier-Stark resonances separated by multiples of the electric field (Freericks, 2008; Freericks and Joura, 2008; Joura, Freericks, and Pruschke, 2008; Tsuji, Oka, and Aoki, 2008; Eckstein and Werner, 2011a, 2013a). Figure 19 shows results for the Falicov-Kimball model at $U=1$. The Wannier-Stark peaks are broadened into bands, whose width is approximately given by $U$. The central peak is split due to interaction effects, which leads to a beating pattern
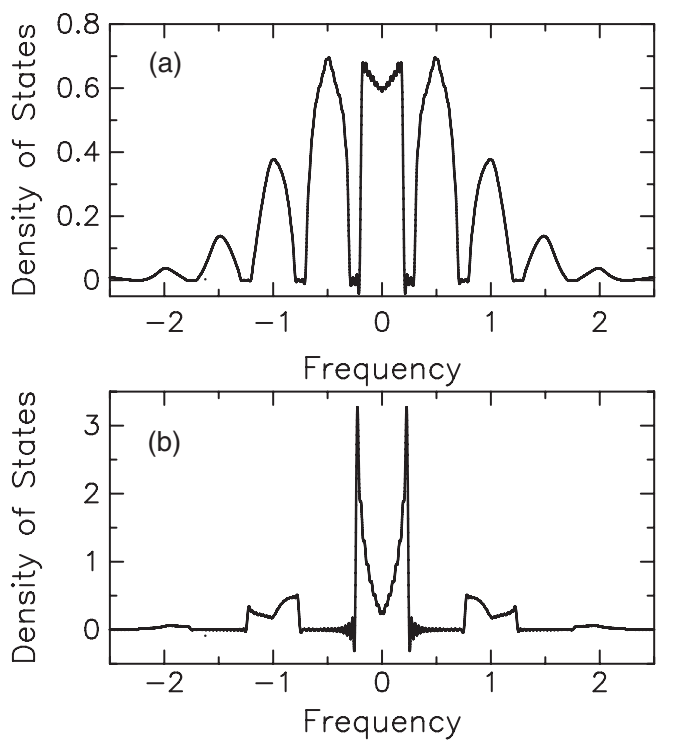

FIG. 19. Many-body density of states of the Falicov-Kimball model with $U=0.5$ and a constant electric field (a) $F=$ 0.5 and (b) $F=1$. From Freericks, 2008.

in the time-dependent current (Freericks, 2008). The interaction effects are effectively enhanced by the electric field, because a steep potential gradient leads to an additional localization of carriers. The extreme limit of this localization is the phenomenon of field-induced dimensional reduction, which occurs when the electric field along one crystallographic direction is so strong that the potential difference between neighboring sites exceeds all other energy scales (Aron, Kotliar, and Weber, 2012).

\section{b. Steady-state current in a dissipative system}

While Bloch oscillations are a typical transient phenomenon, a true stationary state with nonzero current in an interacting system can only be reached when the system is coupled to an external heat bath. [The bath might also be part of the model, as in the situation of a single carrier in a manybody background (Mierzejewski et al., 2011; Golež et al., 2013).] Otherwise, the Joule heating of the system leads to a time-dependent change in the total energy. The dynamics in the dc-driven Hubbard model coupled to a local electron heat bath of the type described in Sec. II.A.2 was studied by Amaricci et al. (2012). Figure 20 shows the time evolution of the current in a Hubbard model on a square lattice with $U=6$ (a strongly correlated metal) after a sudden switch on of a dc field in the diagonal direction. The large initial spike in the current is associated with the buildup of a polarization. Without coupling to the heat bath, the current eventually decays to zero in an oscillatory manner. As the coupling $\Lambda$ to the fermionic heat bath is switched on, the current approaches a nonzero stationary value, because the system relaxes to a nonequilibrium steady state whose momentum distribution is shifted to a position at which the electric field driving is balanced with the dissipative effects from interactions and the heat-bath coupling. The heat bath thus prevents the electrons from reaching an infinite-temperature distribution, so that they contribute to a direct current in the long-time limit. For fixed 


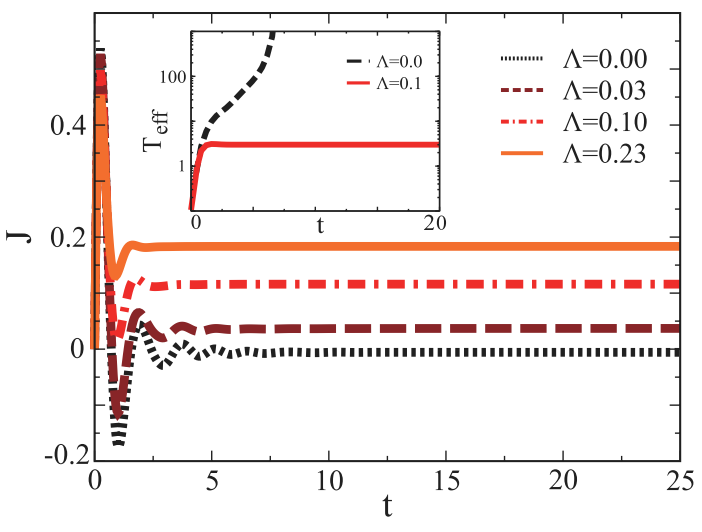

FIG. 20 (color online). dc field-induced current in the Hubbard model with coupling to a thermostat. Current for $U=6$ and $F=$ 4.7 and indicated values of the heat-bath coupling $\Lambda$. The field is suddenly switched on at time $t=0$. The DMFT impurity problem is solved with second-order perturbation theory (IPT). (Inset) Time evolution of the effective temperature for $U=6$ and $F=1.9$. Adapted from Amaricci et al., 2012.

values of $U$ and $\Lambda$, the current is a nonmonotonic function of the field strength: In the weak-field regime one finds Ohmic behavior $(j \propto F)$, whereas in the strong-field regime the current decreases with increasing $F$, because at fixed $\Lambda$ the rate of energy transfer to the bath is limited, while the heat production is proportional to the current and to $F$.

\section{c. Dielectric breakdown}

The previous examples focused on correlated metallic systems subject to a dc field. For interactions larger than approximately the bandwidth, and at low temperatures, the half-filled Hubbard model is in a Mott insulating state. A strong electric field $F$ can lead to a metallization of the Mott insulator via a production of doublon-hole pairs: In the scalar potential gauge, the field gives rise to a potential energy difference $F$ between two neighboring lattice sites in the direction of the field (in units in which the lattice constant $a=1$ and electron charge $e=1$ ). An electron can thus gain the energy $U=\ell_{U} F$ from the field by tunneling over a distance $\ell_{U}$, which will produce a doublon and leave a hole behind [Fig. 21(a)]. The tunneling process is thus expected to contribute a current

$$
\Gamma_{\mathrm{dh}}(t) \sim \ell_{U} \dot{d}(t)
$$

where $\dot{d}$ is the production rate of doublon-hole (dh) pairs.

For $F \ll 1$ the tunneling takes place over many lattice sites, and hence at an exponentially small rate. The scaling for the electric current is expressed, with a threshold field $F_{\text {th }}$, as

$$
j \propto F \exp \left(-F_{\text {th }} / F\right) .
$$

Observables related to the doublon-production rate are indeed found to exhibit this threshold behavior (with different powers of $F$ in the prefactor) in studies of the Hubbard model in one dimension (Oka, Arita, and Aoki, 2003; Oka and Aoki, 2005; Heidrich-Meisner et al., 2010; Oka and Aoki, 2010; Lenarčič and Prelovšek, 2012; Oka, 2012), and in infinite dimensions
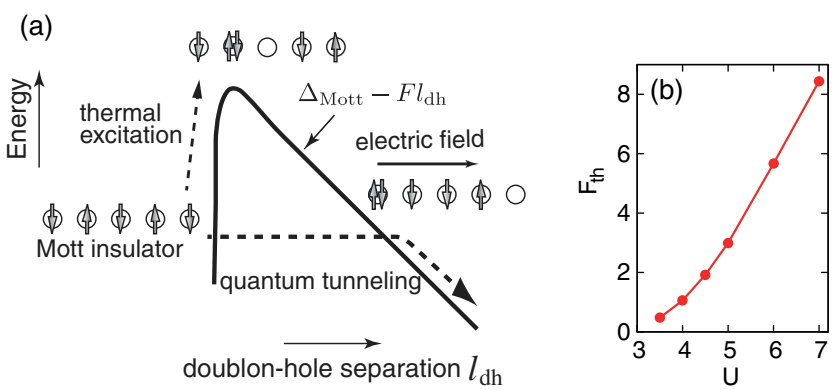

FIG. 21 (color online). (a) Illustration of the dielectric breakdown of a Mott insulator in a strong electric field due to many-body quantum tunneling. The many-body energy (solid curve) is plotted against the separation of doublons and holes $l_{\mathrm{dh}}$. For the dielectric breakdown the state has to tunnel (dashed line) through an energy barrier $\Delta_{\text {Mott }} \sim U$ related to the creation of charge excitations (doublon-hole pairs). Since the electric field reduces the energy of a pair by $F l_{\mathrm{dh}}$, the quantum tunneling among many-body states takes place when $\Delta_{\text {Mott }}-F l_{\mathrm{dh}} \sim 0$, i.e., when $l_{\mathrm{dh}}=\ell_{U}$. The tunneling probability depends on the overlap between the ground state and excited-state wave functions. Adapted from Oka and Aoki, 2009a. (b) The threshold field $F_{\text {th }}(U)$ from DMFT calculations for the hypercubic lattice with density of states $\rho(E) \propto \exp \left(-E^{2}\right)[\mathrm{OCA}$ results from Eckstein, Oka, and Werner (2010)].

(Eckstein, Oka, and Werner, 2010; Eckstein and Werner, 2013a). The steady-state properties of a system coupled to dissipative bath have been studied by Aron (2012). Here we briefly discuss the nonequilibrium DMFT results (Eckstein, Oka, and Werner, 2010; Eckstein and Werner, 2013a) for the half-filled Hubbard model on an infinite-dimensional hypercubic lattice.

In these calculations, which do not involve a coupling to a thermostat, one observes a quasisteady current $j(t)$ at long times which is nonzero and strongly field dependent. The highly nonlinear $j-F$ characteristics obtained from these quasisteady values can be well fitted by Eq. (227), where the threshold field $F_{\text {th }}$ associated with the dielectric breakdown of the Mott insulator extrapolates to zero around the Mott crossover [see Fig. 21(b)]. (Note that the analysis has been performed above the critical temperature.)

Although the current becomes almost stationary at long times, the system is not in a true steady state: energy increases at a rate given by $\dot{E}_{\text {tot }}(t)=\boldsymbol{j}(t) \cdot \boldsymbol{F}(t)$, and also the number of doublons grows almost linearly with time (Eckstein, Oka, and Werner, 2010; Eckstein and Werner, 2013a). A constant current might be surprising at first sight if doublons are viewed as charge carriers, but the observation has a simple interpretation (Eckstein and Werner, 2013a): One finds that the measured current is given almost entirely by the quantummechanical tunneling current (227). This suggests that in the presence of an external field both thermally excited carriers and field-induced carriers rapidly reach an infinite-temperature state (zero kinetic energy), in which their average mobility vanishes. Such a behavior was found for transport in various other isolated systems (Mierzejewski and Prelovšek, 2010; Eckstein and Werner, 2011a). For the Mott insulator, the picture is confirmed by several findings (Eckstein and Werner, 2013a), most notably by (i) an analysis of the occupation function (which becomes flat in the quasisteady state) and (ii), the 
behavior of the current in the presence of a thermostat (carriers now maintain a finite temperature, and the current thus increases with time proportional to the number of field-excited carriers). The scenario is also supported by an analytical calculation performed for a one-dimensional Hubbard model with a nonequilibrium extension of the Bethe ansatz (Oka and Aoki, 2010, Oka, 2012).

We note that the theoretical results obtained from nonequilibrium DMFT and also 1D time-dependent DMRG studies of the Hubbard model do not entirely agree with nonlinear transport experiments. In experiments, a rather strong temperature dependence is seen in the threshold field (Taguchi, Matsumoto, and Tokura, 2000), and a negative differential resistance is observed in many correlated materials (Tokura et al., 1988; Mori et al., 2009). The electron avalanche mechanism also plays an important role (Guiot et al., 2013). The origin of the negative differential resistance is not fully understood yet. It might be explained by different Joule heating scenarios (Altshuler et al., 2009; Mori et al., 2009), or possibly by a nonequilibrium first-order phase transition, where the negative differential resistance is explained through a phase bistability (Ajisaka et al., 2009). A negative differential resistance is also found in a model in high-energy physics, namely, the supersymmetric QCD in the large- $N$ limit (Nakamura, 2010, 2012). It is hence an interesting challenge to develop a microscopic understanding of the nonlinear transport properties of correlated systems from a universal viewpoint.

\section{Photoexcitations and photodoping}

Short laser pulses provide a powerful tool to excite and probe the dynamics of electrons and phonons in correlated materials on the femtosecond time scale. For the excitation one mainly uses (i) mid-IR pulses $(\approx 10-100 \mathrm{THz}$ ), which can control the properties of complex materials by selectively addressing certain optical phonons (Rini et al., 2007; Fausti et al., 2011); (ii) terahertz pulses, which act like static fields on the electron time scale (Hirori, Doi et al., 2011; Watanabe, Minami, and Shimano, 2011; Liu and , 2012); and (iii) pulses in the eV photon-energy range, which can promptly generate electron and holelike carriers (photodoping) (Iwai et al., 2003). Photodoping in correlated materials can induce, e.g., metal-insulator transitions in Mott and charge-transfer insulators, and ultrafast melting of charge and spin order (see the Introduction). Previous studies of photoinduced phase transitions have been performed in 1D using exact diagonalization and time-dependent DMRG for the Hubbard model (Oka and Aoki, 2008; Takahashi, Itoh, and Aihara, 2008), and for the Hubbard-Holstein model (Matsueda et al., 2012) as well as for a spin-charge coupled system (Matsueda and Ishihara, 2007; Kanamori, Matsueda, and Ishihara, 2009, 2011). Nonequilibrium DMFT can potentially simulate the corresponding dynamics in extended higherdimensional systems, and make predictions for time-resolved optical and photoemission spectroscopy (Sec. II.B.5). So far, DMFT was used to study photodoping and the subsequent relaxation in paramagnetic metals and Mott insulators on short times (disregarding lattice dynamics), within the Hubbard model (Eckstein and Werner, 2011b, 2013c) and the Falicov-Kimball model (Moritz, Devereaux, and Freericks, 2010; Moritz et al., 2013). Two important questions in this context, which we discuss in the following, concern the validity of the twotemperature model in this regime, and the formation of quasiparticles after a photoinduced Mott-insulator-to-metal transition.

In simple metals and semiconductors, the photoexcited state can often be understood in terms of the two-temperature model of hot electrons in a colder lattice (Allen, 1987). For this description to work, electron-electron scattering must equilibrate the electrons to a quasiequilibrium state much faster than energy is transferred to the lattice. Thermalization in isolated correlated systems, where simple approaches like the Boltzmann equation fail, is a question of fundamental interest (see Sec. III.B.1). Thermalization after photodoping is observed in the metallic phase of the Hubbard model (Eckstein and Werner, 2011b) and to excellent accuracy even in the Falicov-Kimball model (Moritz, Devereaux, and Freericks, 2010; Moritz et al., 2013), where true thermalization of singleparticle quantities does not occur (Eckstein and Kollar, 2008b). In the Mott phase, on the other hand, the hot-electron picture breaks down (Moritz, Devereaux, and Freericks, 2010; Eckstein and Werner, 2011b; Moritz et al., 2013). As a result, photoemission spectra explicitly depend on the energy distribution of the excitation pulse, and not only on the total amount of absorbed energy (Eckstein and Werner, 2011b).

Figure 22 plots time-resolved spectra of a spinless FalicovKimball model during and after the perturbation by a monocycle pulse. In this calculation, it is assumed that the localized particles are uniformly distributed with a density of 0.5 per site. The unit of energy is given by the hopping $t^{*}$ of the conduction electrons, and at half filling there is a metalinsulator transition at $U_{c}=\sqrt{2} t^{*}$. Figure 22(b) shows the photoemission response of a metallic system $\left(U=0.5 t^{*}\right)$. Here the signal rapidly relaxes back to an almost thermal, but significantally broadened spectrum. In the insulating model [U $=2 t^{*}$; see Fig. 22(c)], the pulse leads to a significant spectral weight transfer across the gap. While the distribution relaxes within the upper and lower bands, there is no relaxation across the Mott gap and the spectral function is not compatible with a thermal distribution.

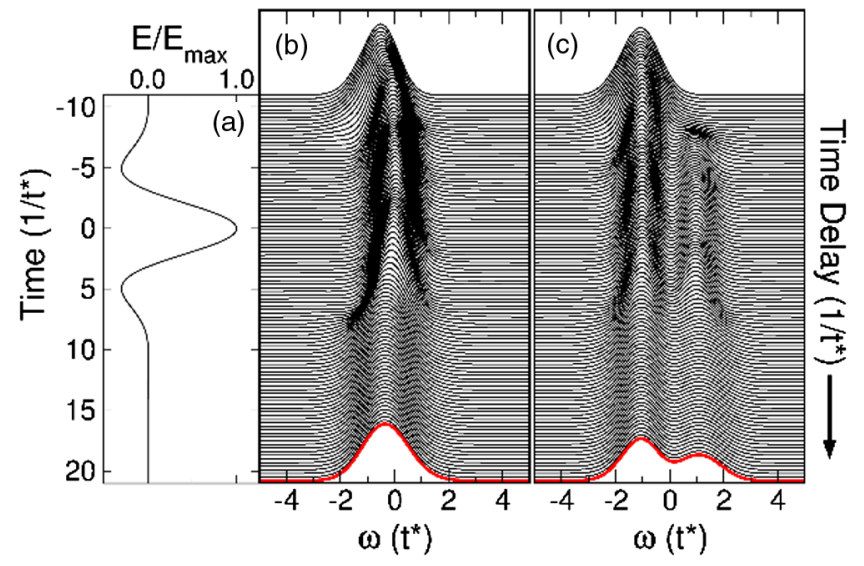

FIG. 22 (color online). Time-resolved photoemission spectrum of the half-filled, spinless Falicov-Kimball model, after excitation by a monocycle pulse. (a) Pulse shape. (b) Metallic system with $U=$ $0.5 t^{*}$. (c) Insulating system with $U=2 t^{*}$. From Moritz et al., 2013. 
The slow thermalization of a photoexcited Mott insulator can be related to the long lifetime of photoexcited doublons and holes, because changing the interaction energy to its quasiequilibrium value requires the creation or annihilation of doublon-holes pairs. This can happen either via the emission of spin excitations (of order $v^{2} / U$ ) or by changing the kinetic energy (of order $v$ ) of other charge excitations. In the Hubbard model, for $U \gg v$, the process involves many scattering partners and is thus exponentially suppressed (Strohmaier et al., 2010; Lenarčič and Prelovšek, 2013). Figure 23(a) shows the evolution of the double occupancy $d(t)$ for a laserexcited Hubbard model at various $U$ in the metal-insulator crossover range $(U \approx 3)$ and the Mott insulating phase (Eckstein and Werner, 2011b). After an initial transient during and after the pulse, $d(t)$ follows an exponential relaxation to a final value $d\left(T_{\text {eff }}\right)$, which can be independently obtained as the thermal expectation value of $d$ in a system with the same energy as the pump-excited one. The time scales extracted from exponential fits to these curves exponentially increase with $U$ [Fig. 23(b)], in agreement with a Fermi-golden-rule type argument (Strohmaier et al., 2010) for high-excitation densities (where the decay to spin excitations is not important).

In the Mott regime, where doublons and holes are stable, a long-lived metallic state is created. Because of its long lifetime up to a few picoseconds, the initially large kinetic energy of photoexcited carriers can be dissipated to phonons before recombination occurs. This raises the intriguing question whether and on what time scale Fermi-liquid quasiparticles might emerge when the kinetic energy is reduced below some coherence scale. A recent DMFT study has investigated this question by coupling the photoexcited Mott insulator at $U \gg v$
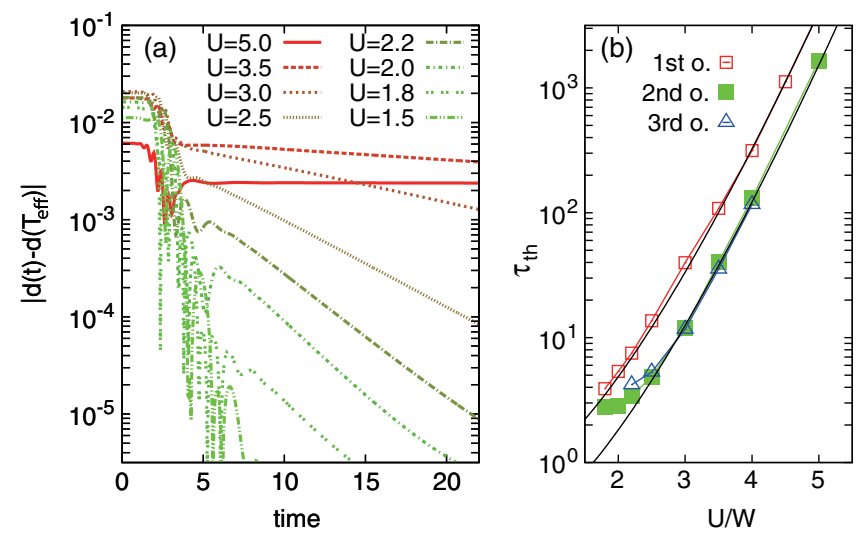

FIG. 23 (color online). (a) Double occupancy in the Hubbard model after photodoping (hypercubic lattice, field along the body diagonal of the lattice; see Sec. II.B.4). The system is excited with a Gaussian field pulse $F(t)=F_{0} \cos (\Omega t) e^{-t^{2} / \Delta t^{2}}$ (frequency $\Omega=U$ ). The initial temperature is $T=0.2$ (above the critical end point of the metal-insulator line), and the field amplitude is chosen such that the final effective temperature is $T_{\text {eff }}=0.5$. (b) Thermalization time, obtained from exponential fits to the data in (a). Solid lines correspond to $\tau \propto \exp [\alpha(U / v) \log (U / v)]$. The strong-coupling expansion (see Sec. II.C.5) was used to solve the DMFT impurity model, and the results for first- (NCA), second(OCA), and third-order (3rd o.) converge. Adapted from Eckstein and Werner, 2011b. to a dissipative environment (Eckstein and Werner, 2013c). Remarkably, a reconstruction of electronic states occurs as the kinetic energy is reduced, the formation of a Fermi-liquid-like state is not observed on accessible time scales.

\section{4. ac electric fields}

\section{a. General remarks}

In this section, we review some recent nonequilibrium DMFT studies of correlated systems driven by time-periodic (ac) electric fields. In contrast to the pulse excitations discussed in Sec. III.A.3, which are designed to induce rapid changes of the states, we consider here time-periodic modulations that allow dynamical, nonequilibrium control of system parameters on a microscopic level. In real solid-state experiments, one has to use pulsed laser fields in order to attain large field intensities. Even in this case, pulses with many oscillation cycles may often safely be regarded as a sinusoidal ac field during irradiation. In the following we give a brief overview of theoretical and experimental works on ac-field problems, and then move on to nonequilibrium DMFT calculations. We distinguish between isolated systems, where the energy accumulates in the system, and open systems, where the energy injected by the external field is balanced by the energy flowing out of the system.

\section{b. Isolated systems}

An isolated system, in which the total energy and the number of particles are conserved, is ideally realized in coldatom experiments, whereas electron systems can be regarded as isolated only on a time scale which is short enough that energy dissipation can be ignored. The effect of ac fields has been theoretically studied for a noninteracting tight-binding model (Dunlap and Kenkre, 1986; Holthaus, 1992) in a timeperiodic electric field $\boldsymbol{E}(t) \equiv \boldsymbol{E} \cos (\Omega t)$. For simplicity, we take a hypercubic lattice in a field along $\boldsymbol{E}=E(1,1, \ldots, 1)$. We can then show that the hopping $v_{i j}$ is renormalized by the periodic driving to an effective hopping (Dunlap and Kenkre, 1986; Holthaus, 1992)

$$
v_{i j}^{\mathrm{eff}}=\mathcal{J}_{0}(A) v_{i j},
$$

where $\mathcal{J}_{0}$ is the zeroth-order Bessel function, and $A=E / \Omega$. Since $\mathcal{J}_{0}$ is an oscillating function, the effective hopping vanishes at zero points $\mathcal{J}_{0}(A)=0$, resulting in immobile particles (dynamical localization). This effect is analogous to the coherent destruction of tunneling in periodically driven two-level systems (Grossmann et al., 1991; Grossmann and Hänggi, 1992).

Equation (228) can be easily understood within the Floquet theory (Sec. II.D). For this we can take the tight-binding Hamiltonian in the temporal gauge [i.e., in terms of the vector potential $\boldsymbol{A}(t)=-\boldsymbol{E} \sin (\Omega t) / \Omega]$ as

$$
H_{0}(t)=\sum_{k} \epsilon_{k-A(t)} c_{k}^{\dagger} c_{k}
$$

where $\epsilon_{k}=-2 v \sum_{\alpha} \cos k_{\alpha} \quad(\alpha=x, y, \ldots)$ is the band dispersion. The Floquet quasienergy of a single-band tightbinding model is given by (Holthaus, 1992; Grifoni and Hänggi, 1998; Tsuji, Oka, and Aoki, 2008) 


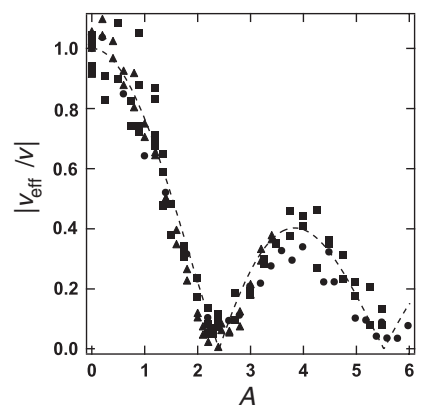

FIG. 24. Dynamical modification of the hopping amplitude observed for Bose-Einstein condensed cold atoms in a periodically shaken optical lattice. $A$ is the ratio of the amplitude to the frequency of the driving ac field and $v_{\text {eff }}(v)$ is the effective (bare) hopping amplitude of particles. From Lignier et al., 2007.

$$
\left\langle\left\langle\varepsilon_{k}\right\rangle\right\rangle-n \Omega \quad(n=0, \pm 1, \pm 2, \ldots),
$$

where $\left\langle\left\langle\epsilon_{\boldsymbol{k}}\right\rangle\right\rangle=\mathscr{T}^{-1} \int_{0}^{\mathscr{T}} d t \epsilon_{\boldsymbol{k}-\boldsymbol{A}(t)}$ is the dispersion averaged over one period of the ac field (see also Sec. II.D.2). For the model with nearest-neighbor hopping [Eq. (229)], we end up with $\left\langle\left\langle\epsilon_{k}\right\rangle\right\rangle=-2 v \sum_{\alpha} \mathcal{J}_{0}(A) \cos k_{\alpha}$, hence Eq. (228). The energy (230) defines Floquet quasiparticles, i.e., the energy dispersion is not only renormalized due to the coupling to the ac field, but a series of Floquet ( $n$-photon dressed) sidebands appears $(n=0, \pm 1, \ldots)$ with a spacing $\Omega$.

The dynamics of systems driven by ac fields has recently been studied in several experiments. Lignier et al. (2007) used Bose-Einstein condensed cold atoms trapped in an optical lattice, where the ac modulation is induced by shaking the lattice potential in real space. They observed the dynamical suppression of the absolute value of the hopping parameter (Fig. 24), in excellent agreement with the predicted Bessel form (228). The coherent control of single-particle tunneling was also reported in a driven double-well system (Kierig et al., 2008). It has been suggested that for interacting systems one can effectively control the dimensionless interaction strength $U / W$ ( $U$ is the on-site interaction and $W$ is the bandwidth) using ac fields, and induce a superfluid-Mott-insulator phase transition in the Bose-Hubbard model (Eckardt, Weiss, and Holthaus, 2005; Creffield and Monteiro, 2006; Zenesini. Lignier, Ciampini et al., 2009). One can even reverse the sign of $v_{i j}$ when $\mathcal{J}_{0}(A)<0$, which was used to realize frustrated classical spin systems on a triangular lattice (Struck et al., 2011).

For fermionic systems, the ac-field problem has been theoretically studied by means of the nonequilibrium DMFT for the half-filled Hubbard model [Eqs. (60) and (61)] (Tsuji et al., 2011). We consider a hypercubic lattice, with the same hopping and field as in Eq. (229). The system is initially in thermal equilibrium, and the ac field is suddenly switched on at $t=0$. Figure 25 shows the result for the double occupancy $d(t)=\left\langle\hat{n}_{\uparrow}(t) \hat{n}_{\downarrow}(t)\right\rangle$ for various values of $A \equiv E / \Omega$ with a fixed $\Omega$. Initially $d$ is smaller than the noninteracting value $\left\langle\hat{n}_{\uparrow}\right\rangle\left\langle\hat{n}_{\downarrow}\right\rangle=0.25$, due to the repulsive interaction. Switching on an ac field with small amplitude leads to a decrease of $d$, accompanied by rapid oscillations with frequency $2 \Omega$ due to the nonlinear effect of the ac field. The suppression of $d$ can be

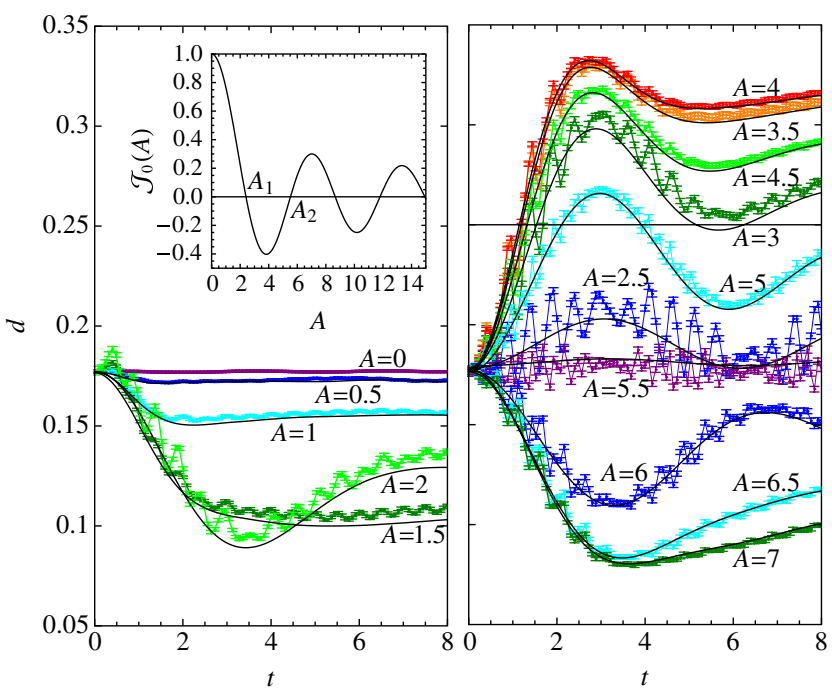

FIG. 25 (color online). Nonequilibrium DMFT result for the time evolution of the double occupancy for the ac-fielddriven Hubbard model (symbols with error bars) with $U=1, \Omega=2 \pi$, and various values of $A \equiv E / \Omega$. Solid curves are the corresponding results for the interaction quench $U \rightarrow U_{\text {eff }}=U / \mathcal{J}_{0}(A)$ with time rescaled as $t /\left|\mathcal{J}_{0}(A)\right|$. The inset shows the Bessel function $\mathcal{J}_{0}(A)$. From Tsuji et al., 2011.

interpreted as coming from an increased $U / W$ due to the hopping renormalization (228).

Remarkably, the double occupancy in Fig. 25 exceeds the noninteracting value of 0.25 in the region where $\mathcal{J}_{0}(A)<0$ (hence $v_{i j}^{\text {eff }}<0$ ). This indicates that the many-body interaction indeed turns into an attraction $\left(U_{\text {eff }}<0\right)$. This ac-fieldinduced attractive interaction may lead to an $s$-wave superconducting state with high $T_{c}$. Note that in equilibrium the inverted sign of $v_{i j}$ does not change the physics due to the particle-hole symmetry. In the present nonequilibrium situation, however, the sign change in $v_{i j}$ between the initial state and the time-evolving state cannot be absorbed by the particle-hole transformation. Physically, it can be interpreted as a dynamical band flipping (Tsuji et al., 2011). As long as the field is ramped up quickly, the occupation in momentum space does not change significantly, resulting in a population inversion, or so-called negative absolute temperature $\left(T_{\text {eff }}<0\right)$ (Klein, 1956; Ramsey, 1956) in the flipped band. If the system thermalizes to the negative-temperature state, the density matrix takes the following form:

$$
\rho(t) \sim e^{-\left[\mathcal{J}_{0}(A) H_{0}+H_{\text {int }}\right] / T_{\text {eff }}}=e^{-\left[H_{0}+H_{\text {int }} / \mathcal{J}_{0}(A)\right] /\left[T_{\text {eff }} / \mathcal{J}_{0}(\mathcal{A})\right]} .
$$

Hence the ac quench amounts to an interaction quench

$$
U \rightarrow U_{\mathrm{eff}}=U / \mathcal{J}_{0}(A)
$$

In other words, a positive (repulsive) $U$ at a negative $T$ translates to a negative (attractive) $U$ at a positive $T$. We can confirm this by comparing the ac-quench results with those of an interaction-quench calculation (solid curves in Fig. 25), where the interaction parameter is quenched as above. For a consistent comparison, time is also rescaled as $t /\left|\mathcal{J}_{0}(A)\right|$ in 
each interaction-quench simulation. We can see that the results for the interaction quench and the ac-field driving agree with each other very well (except for the $2 \Omega$ oscillations).

Recently, the negative absolute temperature state has been realized in an experiment on ultracold bosonic atoms (Braun et al., 2013), following theoretical proposals (Mosk, 2005; Rapp, Mandt, and Rosch, 2010). In real materials, however, it might be difficult to experimentally realize a negative-temperature state using continuous ac fields, since a continuous illumination with an intense laser may result in violent heating. A different proposal for realizing the negativetemperature state is to use a half-cycle or monocycle pulse (Tsuji et al., 2012). If one applies a pulse field $E(t)$ to a noninteracting system, the momentum distribution is shifted according to $k \rightarrow k+\varphi$, where $\varphi=\int_{-\infty}^{\infty} d t E(t)$ is the dynamical phase. If the momentum shift is about $\pi$, one would have a similar inverted population. However, in a usual experimental situation Maxwell's equation dictates that $\varphi$ should vanish. The nonequilibrium DMFT calculation for the interacting system shows that, with an asymmetrically shaped monocycle pulse that satisfies $\varphi=\int_{-\infty}^{\infty} d t E(t)=0$, the manybody interaction can exert different effects for the first half cycle and the second half cycle, so that one may end up with a nonzero shift with an inverted population (Tsuji et al., 2012). Recently, a similar phenomenon has been demonstrated in a cold-atom system, where the complex phase of the hopping $v_{i j}$ was controlled by a train of sinusoidal pulses separated by a certain waiting time (Struck et al., 2012).

\section{c. Open systems}

In real materials, the system of interest is usually coupled to an environment with energy and/or particle dissipation, whose effect should be taken into account in a realistic calculation. When one continuously drives an open system with an ac field, the injection of energy from the external field is balanced by the dissipation of energy into the heat bath, and a nonequilibrium steady state will emerge. This can be thought of as an approximate description of excited states of materials realized during irradiation with a continuous-wave laser or a pulsed laser with many cycles. Theoretically, the nonequilibrium steady state of strongly correlated fermionic systems driven by ac fields has been studied for the FalicovKimball model (Tsuji, Oka, and Aoki, 2008, 2009) and the Hubbard model (Lubatsch and Kroha, 2009) with the nonequilibrium DMFT technique. The Falicov-Kimball model [Eq. (83)] was studied at half filling with a body-diagonal field $\boldsymbol{A}(t)=-\boldsymbol{E} \sin (\Omega t) / \Omega$. The heat bath was modeled by free fermions (Sec. II.A.2.b). One can solve this problem exactly, since the Hamiltonian is quadratic in itinerant fermionic operators (Sec. II.C.2).

Figure 26(a) shows the momentum-resolved single-particle spectral function averaged over one period of the ac field $A(\boldsymbol{k}, \omega)$ (Floquet spectrum), constructed from the gauge invariant Green's function. A salient feature is that, even in the correlated system, Floquet sidebands appear, on top of the original Mott-Hubbard bands, with spacing $\Omega$ [Fig. 26(b)]. In particular, for the Mott insulating state with $\Omega<U$, Floquet sidebands penetrate into the original Mott-Hubbard gap,
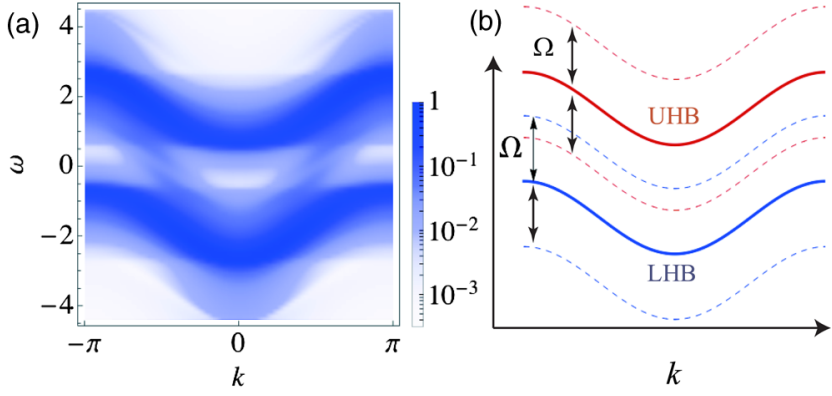

FIG. 26 (color online). (a) The Floquet spectrum $A(\boldsymbol{k}, \omega)$ for the ac-field-driven Falicov-Kimball model with $U=3, E=0.8$, and $\Omega=1.8$. (b) A schematic band structure in the ac field, where the upper (lower) Hubbard band are represented, while their Floquet sidebands with a spacing $\Omega$ are shown by dashed lines. From Tsuji, Oka, and Aoki, 2008.

generating a "photoinduced midgap band" (Tsuji, Oka, and Aoki, 2008).

The optical conductivity $\sigma(\nu)$ has also been calculated for the ac-field-driven Falicov-Kimball model (Tsuji, Oka, and Aoki, 2009) (see Fig. 27). As we increase the amplitude $E$, the charge-transfer peak of the Mott insulator at $\nu \sim U$ collapses due to the bleaching effect. For $\Omega \lesssim U$ [Fig. 27(b)], a broad positive peak appears around $\nu \sim 0$, implying that the system is driven into a bad metal state. For $\Omega>U$ [Fig. 27(c)], a negative optical conductivity appears, which suggests that the system gains energy from the photon. This comes from a

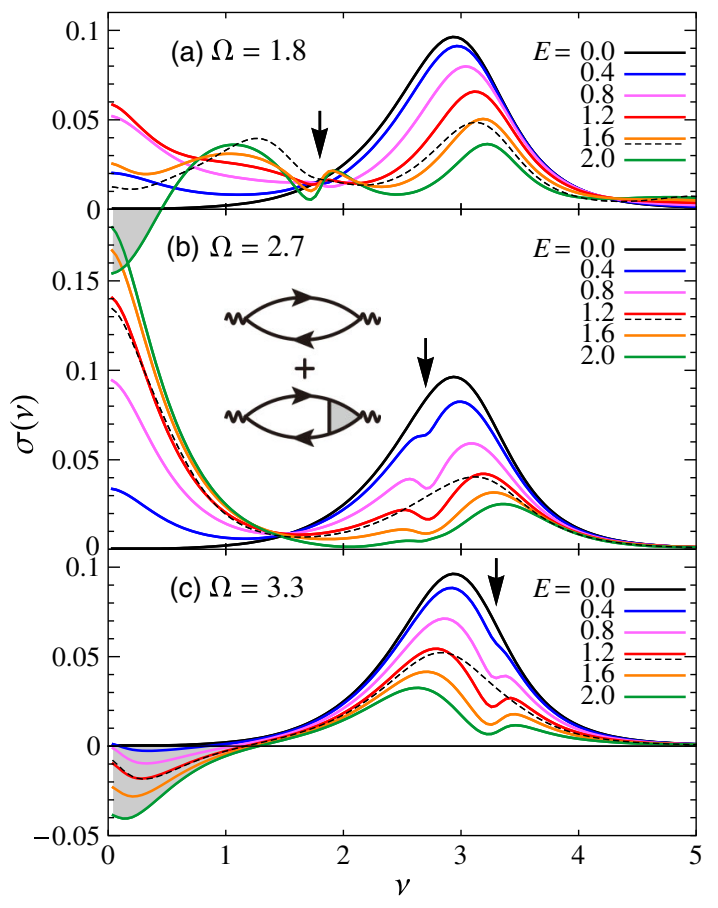

FIG. 27 (color online). Optical conductivity for the ac-fielddriven Falicov-Kimball model coupled to a fermionic heat bath with $U=3, \Gamma=0.05, T=0.05$, and (a) $\Omega=1.8$, (b) 2.7 , and (c) 3.3. The dashed curves illustrate (for specific values of $E$ ) the results without vertex correction. The arrows indicate the frequency $\Omega$ of the ac field. Inset: diagrams of the bubble and vertex correction. From Tsuji, Oka, and Aoki, 2009. 
partial population inversion within the upper (lower) band of the Mott insulator. For $\Omega<U$ [Fig. 27(a)], a midgap absorption is observed at $\nu \sim U-\Omega$, which is attributed to an excitation from the Floquet sidebands to the original band (or vice versa). Another feature in $\sigma(\nu)$ is that there appear kinks [Fig. 27(a)] and dips [Figs. 27(b) and 27(c)] at $\nu \sim \Omega$. By comparing the results with and without vertex correction for the optical conductivity (Fig. 27), we conclude that the vertex correction contributes to $\sigma(\nu)$ significantly around $\nu \sim \Omega$, creating resonancelike spectral structures. Since there is no such correction in the equilibrium DMFT (Khurana, 1990), these features can be considered as a genuine nonequilibrium quantum many-body effect.

\section{B. Time-dependent parameter changes}

In the following sections we discuss two types of parameter changes in isolated many-fermion systems: abrupt quenches and gradual ramps. For quenches the main interest is in the subsequent relaxation of the system, in particular, the question how the system relaxes and whether it thermalizes, as discussed below. The energy is typically conserved after the quench because the evolution of the isolated system is governed by a time-independent Hamiltonian. For ramps the main goal is to understand how the excitation of the system depends on the ramp protocol, e.g., how much energy is transferred to the system and how to minimize it.

\section{Quenches, relaxation, and thermalization}

\section{a. Comparison with the thermal state}

Suppose the initial state is given by the density matrix $\rho(0)$ and the time evolution is determined by the constant Hamiltonian $H$ after the quench. The many-body density matrix

$$
\rho(t)=e^{-i H t} \rho(0) e^{i H t}
$$

will not relax to a stationary limit for long times, but each of its components oscillates forever. On the other hand, quantummechanical expectation values of a large system can relax to steady values, because many oscillating components usually contribute to them. This raises the question of whether the time-dependent expectation values relax to the thermal expectation values as obtained from a standard microcanonical, canonical, or grand-canonical ensemble. If this happens, for momentum-integrated as well as momentum-dependent quantities, the system is said to thermalize. At first glance it is surprising that this should be possible, as the thermal state depends only on the (constant) mean energy and particle number of the system, whereas the time-evolved state may depend on details of the initial state. The so-called eigenstate thermalization hypothesis [see Polkovnikov et al. (2011) for a review] explains this in terms of the observation that for generic interacting systems, expectation values in energy eigenstates usually depend only on the eigenenergy, not on the details of the eigenstate. For integrable systems, on the other hand, a large number of conserved quantities lead to a dependence of expectation values on the individual eigenstates and not just their energy. [Possible criteria for defining integrability in quantum systems have been discussed by Caux and Mossel (2011).] As a consequence, integrable systems usually do not thermalize. However, they can often be described by generalized Gibbs ensembles (GGEs) which take the conserved constants of motion into account (Jaynes, 1957a, 1957b; Rigol, Dunjko, and Olshanii, 2008); for reviews see Dziarmaga (2010) and Polkovnikov et al. (2011). On the other hand, even small integrability-breaking terms in the Hamiltonian can lead to thermalization after sufficiently long times (Rigol, 2009a, 2009b).

b. Interaction quench in the Falicov-Kimball model and the role of conserved quantities

For an interaction quench in the Falicov-Kimball model [see Eq. (83) and Sec. II.C.2] from $U_{-}$to $U_{+}$and hopping $v_{i j}$ corresponding to a semielliptical density of states (69) with $v_{*}=1$, the set of equations (134b) and (134c) can be solved analytically (Eckstein and Kollar, 2008a, 2008b, 2008c). In this setup, a metal-insulator transition occurs at the critical interaction $U_{c}=2$ for half filling, $n_{c}=n_{f}=\frac{1}{2}$. Time-dependent observables such as the double occupancy $d(t)=-i w_{1} R^{<}(t, t)$ and the momentum distribution $n(\epsilon, t)$ [Eq. (111)] relax from their values in the initial state to steady-state values. The energy per site $E=\langle H\rangle / L+\mu n_{c}$ is increased by $\Delta E=$ $\left(U_{+}-U_{-}\right) d\left(0^{-}\right)$at the quench. In general, however, the equilibrium state corresponding to this new energy value is not reached by time evolving the initial state, as discussed below.

Figure 28 shows the double occupation $d(t)$ for different quenches, both within and between the two phases. The relaxation to a new stationary value $d(\infty)$ occurs on the time scale of the inverse hopping, with damped collapse-andrevival oscillations [Fig. 28(c)] after quenches to large interactions. As can be seen from the arrows in Fig. 28, the stationary value $d(\infty)$ differs from the double occupation in the thermal state with the same density and energy. Moreover,

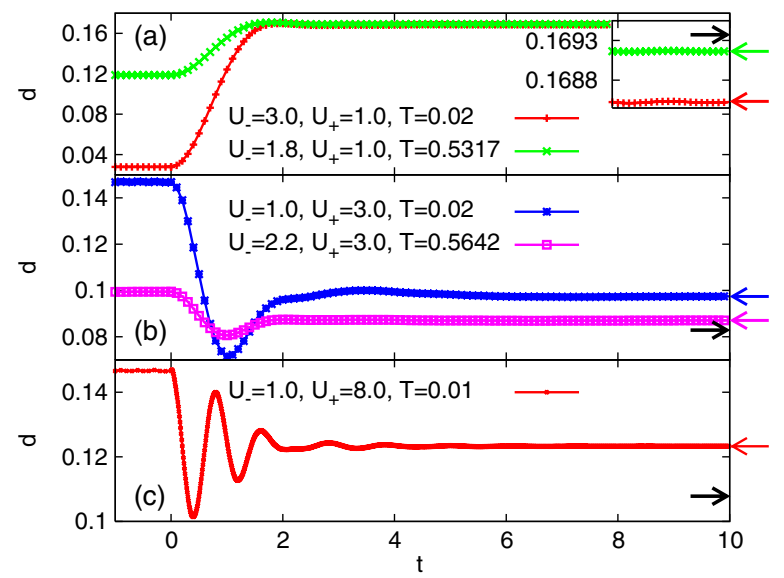

FIG. 28 (color online). Double occupation $d(t)$ for quenches to (a) $U_{+}=1$, (b) $U_{+}=3$, and (c) $U_{+}=8$, starting from an initial metallic $\left(U_{-}<2\right)$ or insulating state $\left(U_{-}>2\right)$. The half bandwidth is 2. For (a) and (b) the energy is the same after both quenches, but the long-time limit (left-pointing arrows) is different for both and also different from the expected thermal value (thick right-pointing arrows). The inset in (a) shows a magnification. From Eckstein and Kollar, 2008b. 
the two initial states in Figs. 28(a) and 28(b) have the same energy after the quench, and thus the same thermal state is expected, but the steady state maintains a more detailed memory of the initial conditions. Thermalization is also lacking for the momentum occupation $n(\epsilon, t)$.

The exact solution of the Falicov-Kimball model after an interaction quench allows one to make analytic statements about the limit of infinite times. One can show for $t \rightarrow \infty$ that the time-dependent occupation function $G^{<}(\omega, t)=$ $\int d s e^{i \omega s} G^{<}(t+s / 2, t-s / 2)$ approaches a steady-state form

$$
g_{\infty}^{<}(\omega)=2 \pi i h(\omega) A_{+}(\omega)
$$

where $A_{+}(\omega)=-i \operatorname{Im}\left[G_{+}^{R}(\omega)\right] / \pi$ denotes the (temperatureindependent) equilibrium spectrum for $U_{+}$. Equation (233) has the same form as the fluctuation-dissipation relation in equilibrium [cf. Eq. (28a)], but the Fermi distribution $f(\omega)$ is replaced by a real and positive function $h(\omega)$, which can be expressed analytically in terms of the initial-state distribution $f(\omega)$ and the equilibrium propagators at $U_{+}$ and $U_{-}$(Eckstein and Kollar, 2008b). The function $h(\omega)$ determines steady-state quantities such as the energy $E(t>0)=\int d \omega h(\omega)(\omega+\mu) A_{+}(\omega)$, the double occupation $d(\infty)=w_{1} \int d \omega h(\omega) \operatorname{Im}\left[r_{+}^{A}(\omega)\right] / \pi$, and the momentum occupation $n(\epsilon, \infty)=\int d \omega h(\omega) \operatorname{Im}\left\{\left[\omega-i 0-\epsilon-\Sigma_{+}^{A}(\omega)\right]^{-1}\right\} / \pi$. In general one finds $h(\omega) \neq f(\omega)$, which is evidence for the absence of thermalization in the Falicov-Kimball model.

This lack of thermalization implies that either the $f$ or the $c$ particles (or both) do not reach their thermal state. The equilibrium $f$ occupation numbers correspond to annealed disorder, but in the paramagnetic phase in DMFT the occupation numbers $\boldsymbol{n}_{f} \equiv\left\{f_{i}^{\dagger} f_{i}\right\}$ are independently distributed on all sites for all temperatures. Therefore the observed nonthermal steady state must be attributed to the lack of thermalization of the $c$ particles, and indeed, the $c$ Hamiltonian is quadratic for given $\boldsymbol{n}_{f}$. After diagonalization one obtains a set of single-particle states $\left|\alpha\left[\boldsymbol{n}_{f}\right]\right\rangle$ and the occupation numbers $n_{\alpha\left[\boldsymbol{n}_{f}\right]}$ are entirely determined by their equilibrium values before the quench. Nonthermal steady states are therefore expected. For infinitesimal interaction quenches $\delta U$, a GGE built from the conserved $n_{\alpha\left[\boldsymbol{n}_{f}\right]}$, averaged over $\boldsymbol{n}_{f}$ with the statistical weight taken from the initial state, provides a correct prediction of the final steady state with Green's function $g_{\infty}^{<}(\omega)+\delta g_{\infty}^{<}(\omega)$. The general scenario of nonthermal steady states and their description by GGEs, originally developed for one-dimensional integrable models, thus also applies to the DMFT solution of the FK model, i.e., for infinite-dimensional lattices.

c. Interaction quench in the Hubbard model, prethermalization and thermalization

The Hubbard model [Eqs. (60) and (61)] at half filling, with a time-dependent interaction term, was studied using nonequilibrium DMFT by Eckstein, Kollar, and Werner (2009, 2010) for the paramagnetic phase and a semielliptic density of states (69) with $v_{*}=1$. The system is prepared in the ground state of the noninteracting Hamiltonian, i.e., $U(t<0)=0$. At $t=0$ the Coulomb repulsion is switched to a finite value, $U(t \geq 0)=U$. The impurity problem is solved with CTQMC, and observables of the lattice model are computed as described in Sec. II.B.5. For the noninteracting initial state the weak-coupling method has the advantage that the imaginary branch of the contour does not enter the CTQMC calculation, so that even zero-temperature initial states can be studied.

Figure 29 shows the momentum distribution $n\left(\epsilon_{k}, t\right)=$ $\left\langle c_{k, \sigma}^{\dagger}(t) c_{k, \sigma}(t)\right\rangle$ for different final values of $U$ as a function of the band energy $\epsilon \equiv \epsilon_{k}$. It evolves from a step function for the initial Fermi sea toward a continuous function of $\epsilon$. The discontinuity $\Delta n$ at $\epsilon=0$ does not disappear at once, rather it remains sharp while its size decreases with time. This decrease is directly related to the decay of electron and hole excitations which are created at time $t=0$ at the Fermi surface. This follows from the equation

$$
\Delta n(t)=n\left(0^{-}, t\right)-n\left(0^{+}, t\right)=\left|G_{\varepsilon=0, \sigma}^{R}(t, 0)\right|^{2}
$$

(valid for a noninteracting initial state at half filling), where $G_{\epsilon_{k}, \sigma}^{R}(t, 0)$ is the retarded component of the momentumresolved Green's function. The double occupation $d$ and the discontinuity $\Delta n$ are shown as a function of the time after the quench in Fig. 30.

Three different regimes are apparent in the relaxation after the interaction quench: small and large values of $U$, separated by a sharp crossover or transition near the intermediate scale $U \approx 3.2=U_{c}^{\text {dyn }}$. Near $U=U_{c}^{\text {dyn }}$, the momentum distribution quickly relaxes to the thermal distribution for all energies $\epsilon$ (solid line in Fig. 29, obtained from a grand-canonical DMFT
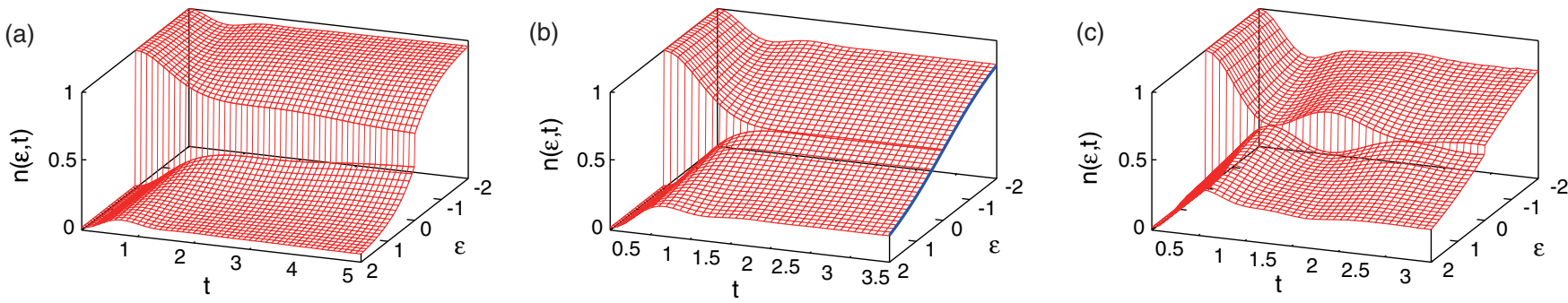

FIG. 29 (color online). Momentum distribution $n(\varepsilon, t)$ after an interaction quench in the Hubbard model from the noninteracting ground state $(U=0)$ to interaction (a) $U=2$, (b) $U=3.3$, and (c) $U=5$; the half bandwidth is 2 . The solid line at $t=3.5$ in (b) is the equilibrium expectation value for the momentum distribution at the same total energy (temperature $T=0.84$ ). Adapted from Eckstein, Kollar, and Werner, 2010. 

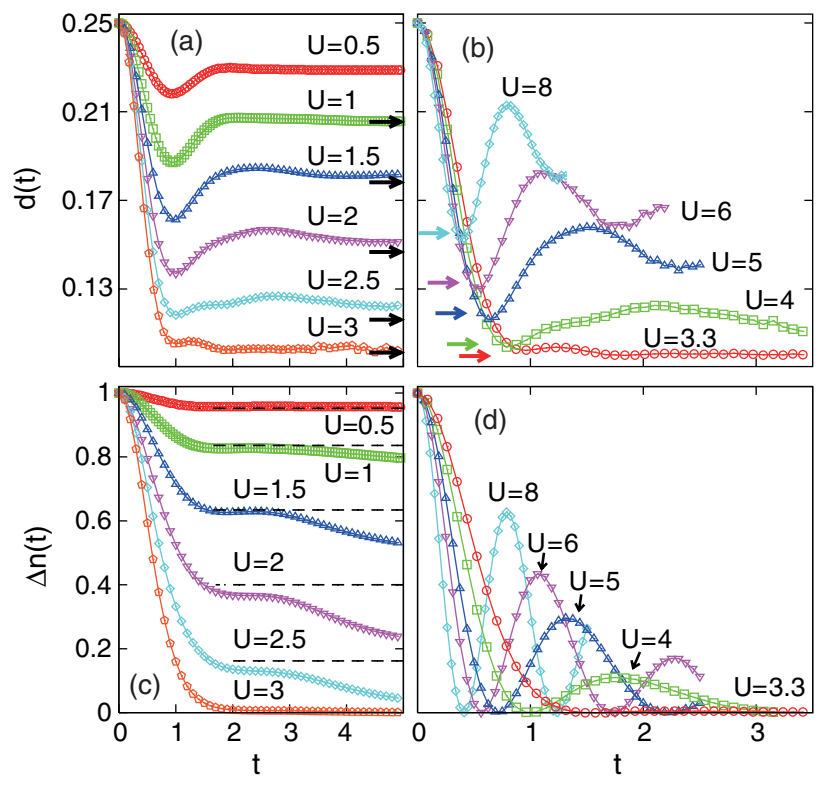

FIG. 30 (color online). Fermi surface discontinuity $\Delta n$ and double occupation $d(t)$ after quenches to $U \leq 3$ (left panels) and $U \geq 3.5$ (right panels). Horizontal dotted lines in panel (c) are the prethermalization plateaus predicted by Moeckel and Kehrein (2008). Horizontal arrows indicate thermal values of the double occupation. Adapted from Eckstein, Kollar, and Werner, 2009.

equilibrium calculation for the temperature that gives the same total energy $E$ ), and thermalization on the same time scale of $\approx 2$ is observed for the double occupancy [thick arrows in Figs. 30(a) and 30(b)], as well as for dynamical observables like the retarded Green's function $G^{R}(t+s, t$ ) (as a function of time difference $s$ ) and the two-time optical conductivity $\sigma(t, t+s)$ (Eckstein, Kollar, and Werner, 2010). After the relaxation it is thus appropriate to regard the system as thermalized, establishing the infinite-dimensional Hubbard model as one of the few isolated quantum many-body systems for which thermalization can be demonstrated [see Polkovnikov et al. (2011) for other examples].

For quenches to interactions $U$ above and below $U_{c}^{\text {dyn }}$ the system does not relax directly to a thermal state. Rather, metastable states are observed on intermediate time scales. For quenches to weak coupling, $U \leq 3$, the double occupation $d(t)$ relaxes from its initial uncorrelated value $d(0)=1 / 4$ almost to its thermal value $d_{\text {th }}$, whereas the Fermi surface discontinuity $\Delta n(t)$ approaches a quasistationary value and remains finite for $t \leq 5$. This so-called prethermalization (Berges, Borsányi, and Wetterich, 2004) was predicted for a quenched Fermi liquid by Moeckel and Kehrein (2008) on the basis of a weak-coupling calculation: while the kinetic and potential energies thermalize on time scales $1 / U^{2}$, the Fermi surface continuity only reaches the plateau $\Delta n_{\text {stat }}=1-2 Z$, where $Z$ is the quasiparticle weight in equilibrium at zero temperature. The momentum occupations are then redistributed as the thermal state is approached. In the limit of infinite dimensions for a half-filled symmetric band the weakcoupling results of Moeckel and Kehrein (2008) for the transient toward the prethermalization plateau describe the transient behavior and the prethermalization plateau well for
$U \lesssim 1.5$ (Eckstein, Kollar, and Werner, 2009), even though at the larger $U$ values the time scales $1 / U^{2}$ and $1 / U^{4}$ are no longer well separated. Prethermalization plateaus after an interaction quench are also correctly predicted by a GGE built from approximate constants of motion (Kollar, Wolf, and Eckstein, 2011). Recently a quantum kinetic equation was used to describe the subsequent crossover from the prethermalization plateau to the thermal state (Stark and Kollar, 2013).

For quenches to strong coupling $[U \geq 3.3$ in Figs. 30(b) and $30(d)]$, the relaxation exhibits damped collapse and revival oscillations of approximate periodicity $2 \pi / U$, due to the exact periodicity of the propagator $e^{-i H t}$ without hopping (Greiner et al., 2002). For large values of $U$ both $d(t)$ and $n(\epsilon, t)$ oscillate around nonthermal values. Using strong-coupling perturbation theory, Eckstein, Kollar, and Werner (2009) showed that the mean value of $d(t)$ for these oscillations is $d_{\text {stat }}=d(0)-\Delta d$ with $\Delta d=(1 / 2 U)\left\langle H_{\text {kin }} / L\right\rangle_{t=0}$ (for the quench from $U=0$, in infinite dimensions at half filling). The thermal value is obtained from a high-temperature expansion as $d_{\text {th }}=d(0)+(1 / U)\left\langle H_{\text {kin }} / L\right\rangle_{0}$. Hence during the initial stage of the relaxation the double occupation relaxes only halfway toward $d_{\text {th }}$. Although longer times cannot be accessed with the weak-coupling CTQMC method, a relaxation to the thermal state is expected after the oscillations have decayed, as in the case of a pump-excited Mott insulator (Eckstein and Werner, 2011b). In general this crossover will set in only on times scales that are exponentially large in the interaction $U$ (Sensarma et al., 2010); see also Sec. III.A.3.

The rapid thermalization at $U \approx U_{c}^{\mathrm{dyn}}$ occurs at the border between the delayed thermalization due to either weakcoupling prethermalization plateaus or strong-coupling oscillations around nonthermal values. Indeed, no finite width was detected for the width of this crossover region, so that a dynamical phase transition might occur at $U_{c}^{\text {dyn }}$. This sharp crossover was unexpected because the corresponding equilibrium temperature $T_{\text {eff }}$ after the quench is much higher than the critical end point of the Mott metal-insulator transition in equilibrium $\left[T_{c} \approx 0.055\right.$ (Georges et al., 1996), but $T_{\text {eff }}=$ 0.84 for $U=3.3$ ]. Interestingly, a good approximation for the critical interaction $U_{c}^{\mathrm{dyn}} \approx 3.4$ is obtained from a timedependent variational theory using the Gutzwiller approximation (Schiró and Fabrizio, 2010, 2011). A similar strong dependence on the quenched interaction was observed in Heisenberg chains (Barmettler et al., 2009). Several possible origins for nonequilibrium phase transitions have been proposed (Sciolla and Biroli, 2010; Gambassi and Calabrese, 2011; Hamerla and Uhrig, 2013; Heyl, Polkovnikov, and Kehrein, 2013; Karrasch and Schuricht, 2013).

\section{d. Interaction quench in the presence of long-range order}

Correlated lattice systems exhibit various types of longrange order including antiferromagnetism, superconductivity, and charge order, in the presence of which the relaxation behavior after an interaction quench changes qualitatively. A symmetry-broken state on a bipartite lattice can be treated within DMFT by solving impurity problems for each sublattice (Georges et al., 1996). For the antiferromagnetic phase and the semielliptic density of states (69), the hybridization 
function $\Delta_{A, \sigma}\left(\Delta_{B, \sigma}\right)$ for the $A(B)$ sublattice is given by the self-consistency condition $\Delta_{A, \sigma}=v_{*}^{2} G_{B, \sigma} \quad\left(\Delta_{B, \sigma}=v_{*}^{2} G_{A, \sigma}\right)$, where $G$ is the local lattice Green's function. Together with the relation $\Delta_{A, \sigma}=\Delta_{B, \bar{\sigma}}$ (for pure Néel-type symmetry breaking), this leads to a single impurity calculation with the self-consistency $\Delta_{\sigma}=v_{*}^{2} G_{\bar{\sigma}}$.

The DMFT phase diagram for the half-filled, repulsive Hubbard model exhibits an antiferromagnetically ordered insulating phase at low temperature (denoted by AFM in the top panel of Fig. 31). For attractive $U$, one finds an analogous phase diagram with AFM order replaced by $s$-wave superconductivity (Micnas, Ranninger, and Robaszkiewicz, 1990; Keller, Metzner, and Schollwöck, 2001) [at half filling the superconducting state is degenerate with a charge-ordered phase due to a symmetry between the repulsive and attractive models (Shiba, 1972), but in the doped system, superconductivity is more stable]. The nature of the AFM insulating (or $s$-wave superconducting) state changes qualitatively as $|U|$ crosses the value corresponding roughly to the maximum in the critical temperature. This is known as the BCS-BEC crossover in the literature on cold atomic gases. Besides the exact CTQMC result (Koga and Werner, 2011), Fig. 31 shows the phase boundaries obtained using the third-order
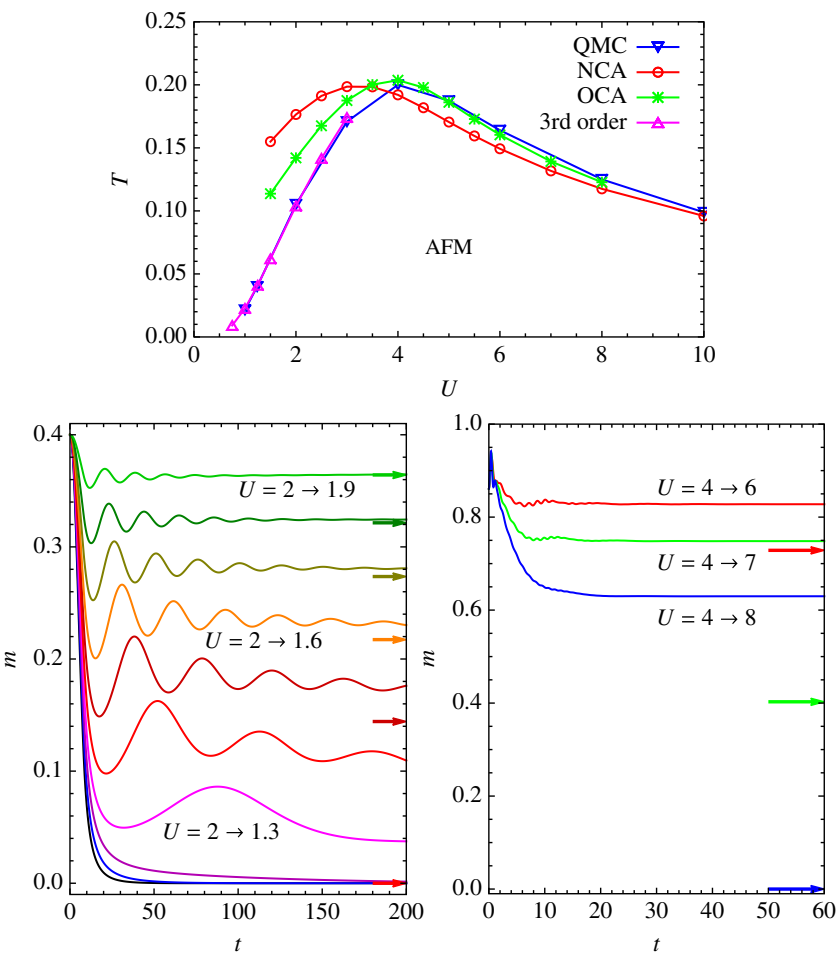

FIG. 31 (color online). Top panel: Antiferromagnetic phase diagram for the half-filled Hubbard model (semielliptic DOS, bandwidth 4). The QMC data are from Koga and Werner (2011), the NCA and OCA phase-boundaries from Werner, Tsuji, and Eckstein (2012), and the third-order weak-coupling perturbation results from Tsuji, Eckstein, and Werner (2013). The time evolution of the order parameter (staggered magnetization) is shown for quenches $U=2 \rightarrow 1.9,1.8, \ldots, 1.0$ [bottom left, from Tsuji, Eckstein, and Werner (2013)], and for quenches $U=4 \rightarrow 6,7$, 8 [bottom right, from Werner, Tsuji, and Eckstein (2012)]. The arrows indicate the corresponding thermal values of the order parameter reached in the long-time limit. weak-coupling perturbation theory (II.C.4), as well as the first- and second-order strong-coupling perturbation theory (NCA and OCA) (II.C.5) as an impurity solver. These solvers have been used by Tsuji, Eckstein, and Werner (2013) and Werner, Tsuji, and Eckstein (2012) to compute the dynamics of the order parameter after interaction quenches from the AFM insulating phase into the paramagnetic metallic phase.

In the bottom left panel of Fig. 31, we plot the time evolution of the magnetization $m$ after quenches from $U_{i}=2$ to $U_{f}=1.9,1.8, \ldots, 1.0$ (Tsuji, Eckstein, and Werner, 2013). These nonequilibrium DMFT calculations are based on thirdorder weak-coupling perturbation theory (II.C.4). The arrows indicate the values of the magnetization expected for the thermalized state in the long-time limit. After the quench, the order parameter shows coherent oscillations (amplitude mode), followed by a slow decay. A remarkable observation is that even though the system, after quenches to $U_{f}<1.5$, is highly excited and will eventually relax to a high-temperature paramagnetic state, the magnetization oscillates for a long time around a nonzero value. This nonthermal magnetized state persists up to a nonthermal critical point, where the frequency of the amplitude mode vanishes, and the dephasing time constant diverges (Tsuji, Eckstein, and Werner, 2013). The behavior of the system in the trapped state is similar to the Hartree solution, which is mathematically equivalent to an integrable BCS equation (Barankov and Levitov, 2006; Yuzbashyan and Dzero, 2006), so that the system may be considered as evolving in the vicinity of the nonthermal Hartree fixed point. The slow relaxation of the nonthermal order is followed by a faster thermalization process.

Trapping phenomena of a different origin are found in quenches to large $U$. The bottom right panel of Fig. 31 shows the magnetization for quenches from $U_{i}=4$ to $U_{f}=6,7$, and 8 , obtained from the nonequilibrium DMFT with the NCA impurity solver (Werner, Tsuji, and Eckstein, 2012). Again the magnetization does not immediately decay to zero after a quench to $U=8$, but remains trapped at a remarkably large value. The state after such a quench is similar to a photodoped state, and the trapping of the magnetization is linked to the exponentially long lifetime $\left(\sim A e^{\alpha(U / 2) \log (U / 2)}\right)$ of artificially created doublons in a Mott insulator with large gap (see also Sec. III.A.3). If the density of frozen-in (or photodoped) carriers is larger than some critical value, the trapping disappears, and the magnetization relaxes to zero exponentially, with a relaxation time which depends like a power law on the distance from the trapped phase (Werner, Tsuji, and Eckstein, 2012).

\section{Ramps and nonadiabaticity}

\section{a. Excitation energy after a continuous parameter change}

The intention in the study of continuous parameter changes (ramps) is often to excite an isolated quantum system as little as possible. The adiabatic theorem of quantum mechanics (Born and Fock, 1928; Kato, 1950; Avron and Elgart, 1999) states that in the limit of infinitely slow changes the system [described by a pure state $\rho(t)=|\psi(t)\rangle\langle\psi(t)|$ ] remains in the ground state of the Hamiltonian $H(t)$. However, for ramps that take place in a finite time $0 \leq t \leq \tau$, the excitation energy, defined as 


$$
\Delta E(\tau)=E(\tau)-E_{0}(\tau),
$$

is positive in general and can only be zero if the final timeevolved state $|\psi(\tau)\rangle$ is a ground state of the final Hamiltonian $H(\tau)$. Here $E(t)=\langle\psi(t)|H(t)| \psi(t)\rangle$ is the energy at time $t$ and $E_{0}(t)$ is the ground-state energy of $H(t)$. Note that the energy $E(t)$ is thus always well defined, in contrast to the entropy, which is defined only for equilibrium states. In equilibrium thermodynamics, the entropy remains constant during adiabatic processes (defined as quasistatic processes without heat exchange with the environment), but increases for processes that take place in a finite time and are hence no longer reversible. The simplest nontrivial quantum system that illustrates the crossover from adiabatic to nonadiabatic behavior is the exactly solvable Landau-Zener model (Landau, 1932; Zener, 1932): a two-level system $H_{\mathrm{LZ}}(t)=v t \sigma_{z}+\gamma \sigma_{x}$ that is driven through an avoided level crossing with finite speed $v>0$ ( $\sigma_{i}$ denote the Pauli matrices). If the system is in the ground state $\left|\phi_{0}(-\infty)\right\rangle=\left(\begin{array}{l}1 \\ 0\end{array}\right)$ at time $t=-\infty$, the probability $p$ to find the system in the excited state $\left|\phi_{1}(\infty)\right\rangle=\left(\begin{array}{l}1 \\ 0\end{array}\right)$ at time $t \rightarrow \infty$ vanishes exponentially when the speed $v$ is small compared to the scale $\gamma^{2} / \hbar$ set by the gap $\gamma$ at the avoided crossing, $p \sim \exp \left(-\pi \gamma^{2} / v \hbar\right)$. This prediction was recently confirmed in cold-atom experiments with accelerated optical lattices (Zenesini, Lignier, Tayebirad et al., 2009). An analogous mechanism also explains the amount of energy injected into a system upon crossing a quantum-critical point or parameter changes in a gapless phase (Dziarmaga, 2010), as obtained from adiabatic perturbation theory (Grandi and Polkovnikov, 2010). In these cases the excitation energy typically behaves as $\Delta E(\tau) \sim \tau^{-\eta}$ for large ramp times $\tau \rightarrow \infty$, with the positive exponent $\eta$ depending on the details of the system and the ramp protocol. The behavior can be understood in terms of the Kibble-Zurek mechanism (Dziarmaga, 2010): The dependence of $\Delta E$ on $\tau$ is due to excitations that are frozen in when the rate of change of the Hamiltonian exceeds the fastest possible relaxation rate of the system (estimated by the energy gap divided by $\hbar$ ).

As a generic case, suppose a system is in the ground state of a Hamiltonian $H_{0}$ at time $t=0$ and a ramp protocol is given by the Hamiltonian $H(t)=H_{0}+\delta \kappa r(t / \tau) W$. Here the ramp shape $r(t)$ starts at $r(0)=0$ and ends at $r(1)=1$, i.e., after the ramp time $\tau$ the operator $\delta \kappa W$ has been added to the Hamiltonian $H_{0}$, where $\delta \kappa$ determines the strength of the perturbation. If no phase boundary is crossed by this ramp and the magnitude of the ramp is small, the excitation energy can be estimated from lowest-order adiabatic perturbation theory (APT) as (Eckstein and Kollar, 2010)

$$
\begin{gathered}
\Delta E(\tau)=\delta \kappa^{2} \int_{0}^{\infty} \frac{d \omega}{\omega} R(\omega) F(\omega \tau)+\mathcal{O}\left(\delta \kappa^{3}\right), \\
R(\omega)=\frac{1}{L} \sum_{n \neq 0}\left|\left\langle\phi_{n}|W| \phi_{0}\right\rangle\right|^{2} \delta\left(\omega-\epsilon_{n 0}\right), \\
F(x)=\left|\int_{0}^{1} d s r^{\prime}(s) e^{i x s}\right|^{2},
\end{gathered}
$$

in terms of the initial spectrum, $H_{0}\left|\phi_{n}\right\rangle=\epsilon_{n 0}\left|\phi_{n}\right\rangle$. The correlation function $R(\omega)$ measures the spectral density of excitations created by $W$ and depends neither on the ramp time $\tau$ nor on the ramp shape $r(x)$, while the ramp spectrum $F(x)$ depends only on the ramp shape. In particular for fast ramps, $\tau \lesssim \tau_{\text {quench }} \approx 1 / \Omega$, where $\Omega$ is the bandwidth of $R(\omega)$, the ramp shape does not matter, as one can then replace $F(\omega \tau)$ by $F(0)=1$ in Eq. (236). On the other hand, for large $\tau$ and continuous ramps, $F(\omega \tau)$ develops a peak at $\omega=0$ and its finite width is responsible for the positive excitation energy $\Delta E(\tau)$. In general, smoother ramps lead to a faster decay of $F(x)$. For a continuous ramp without finite steps, $F(x)$ falls off at least as $1 / x^{2}$. The simple linear ramp $r_{1}(x)=x$ corresponds to $F_{1}(x)=2[1-\cos (x)] / x^{2}$, but this can usually be improved by making one or more of its derivatives continuous (Eckstein and Kollar, 2010), or by allowing $r(x)$ to oscillate (Eurich, Eckstein, and Werner, 2011).

\section{b. Linear ramps in the Falicov-Kimball model}

The solution for quenches in the Falicov-Kimball model [Eq. (83)] can also be adapted for continuous changes $v(t)$ or $U(t)$ (Eckstein and Kollar, 2010). For a semielliptic density of states a Mott metal-insulator transition occurs at $U_{c}=2 v_{*}$ in equilibrium DMFT, and for a time-dependent bandwidth [Eq. (69) with $v_{*}=v(t)$ ] the self-consistency equation is given by Eq. (103).

For linear ramps of the hopping parameter from $v_{i}$ to $v_{f}$ the resulting excitation energy depends on the thermodynamic phase, namely (Eckstein and Kollar, 2010),

$$
\Delta E(\tau) \stackrel{\tau \rightarrow \infty}{\sim} \begin{cases}\tau^{-\frac{1}{2}} & \text { across the transition, } \\ \tau^{-1} & \text { in the metallic phase, } \\ \tau^{-2} & \text { in the insulating phase. }\end{cases}
$$

Here the exponents for ramps inside one of the phases can be explained in terms of the perturbative result (236). For an insulating initial state, the function $R(\omega)$ can be approximated by a sharp peak near $U$ due to the charge gap $R(\omega) \propto$ $\delta(\omega-U)$, and hence $\delta E(\tau) \propto F_{1}(\omega \tau) \propto \tau^{-2}$. This indicates that for such a ramp the excitation as a function of $\tau$ strongly depends on the ramp shape, which is confirmed by the numerical analysis. Also the asymptotic power-law behavior for large $\tau$ is then determined by the ramp shape rather than by intrinsic properties of the system.

For ramps inside a gapless phase, on the other hand, not only the ramp shape $r(x)$, but also the excitation spectrum $R(\omega)$ matters. Following Eckstein and Kollar (2010), we suppose a ramp is performed inside the metallic phase of the FK or, for comparison, the Hubbard model, i.e., starting from $U=0$ we turn on a (small) interaction $U$ linearly during the ramp time $\tau$. In DMFT it follows from perturbation theory in $U$ that for this ramp $R(\omega) \propto \omega$ for the FK model, while $R(\omega) \propto \omega^{3}$ for the Hubbard model, i.e., more excitations are created in the former non-Fermi liquid than the latter Fermi liquid. It now depends on the ramp shape whether this behavior $R(\omega) \sim \omega^{\nu}$ can be observed. Suppose the ramp shape is such that $F(x) \sim 1 / x^{\alpha}$ (e.g., $\alpha=2$ for the linear ramp). Then only for sufficiently smooth ramps with $\alpha>\nu$ the excitation energy is indeed given by $\Delta E \sim \tau^{-\nu}$, otherwise the intrinsic exponent $\nu$ is hidden and $\Delta E \sim \tau$ is determined by $\alpha$. 
Hence for the FK model, any continuous interaction ramp leads to $\Delta E \sim \tau^{-1}$. On the other hand, for the Hubbard model the excitations caused by a linear ramp, $\Delta E \sim \tau^{-2}$ [also obtained by Moeckel and Kehrein (2010)], already mask the intrinsic behavior of $R(\omega)$. This universal dependence of the excitation energy on the ramp time due to the ramp shape was also discussed for a variety of systems by Haque and Zimmer (2013).

\section{c. Oscillating ramps in the Hubbard model}

Interaction ramps from 0 to $U$ with arbitrary ramp shapes for the Hubbard model in DMFT were studied by Eurich, Eckstein, and Werner (2011), with the aim of minimizing the excitation energy by suitably shaping the ramp for a given ramp time $\tau$. By optimizing Eq. (236) it was found that ramps with oscillations $r(x)=x+a \sin (2 \pi n x)$ can lead to a lower excitation energy $\Delta E$ than linear ramps, where $n$ is an integer and $a$ is on the order of unity. For such ramps the function $F(x)$ first decays from $F(0)=1$ for small $x$, then grows again to large values for larger $x$. Nevertheless the excitation energy remains small because $R(\omega)$ has a finite bandwidth and, depending on $\tau$, may collect only a part where $F(\omega \tau)$ is small, as supported by DMFT weak-coupling CTQMC data, which agree with the APT estimates [Eq. (236)]. Physically this means that if one cannot take sufficient time to slowly change the Hamiltonian, it may in some cases be better to change it so quickly that the system cannot follow at all.

In practice, not only a small excitation energy may be desirable, but also a speedy thermalization after the ramp is finished. Eurich, Eckstein, and Werner (2011) found that a similar critical value of $U_{c}$ for rapid thermalization exists as in the case of a sudden quench (Sec. III.B.1), for which rapid thermalization occurs after the ramp. For fixed ramp time $\tau=1.25$ they obtained $U_{c}^{\text {linear }} \approx 3.75$ for linear ramps, while $U_{c}^{\text {oscillating }} \approx 4.25$ for oscillating ramps with $r(x)=$ $x+0.87 \sin (4 \pi x)$. This may be compared to variational results by Sandri, Schiró, and Fabrizio (2012), who found that $U_{c}$ increases toward the equilibrium critical value for the Mott transition as $\tau$ tends to infinity. The study of ramps may thus help to understand the relation between the critical interaction values in equilibrium and nonequilibrium.

\section{CONCLUDING REMARKS AND PROSPECTS}

We have given an overview of the nonequilibrium DMFT formalism for the study of nonequilibrium phenomena in correlated fermionic lattice systems. While the extension of the DMFT formalism to nonequilibrium situations involves, formally, only the replacement of the imaginary-time interval with a suitable contour, we have shown that the solution of the DMFT equations on this contour is different and requires significantly more elaborate techniques. While in thermal equilibrium the Green's functions and self-energies are timetranslation invariant, these quantities depend on two time variables in nonequilibrium, and the DMFT equations either acquire an additional Floquet matrix structure (for periodically driven systems) or they become integral-differential equations on the contour (for temporal evolutions after a disturbance). The integral-differential equations are best solved by implementing a step-by-step propagation on the real-time axis, starting from some equilibrium DMFT solution. Furthermore, while distribution functions and spectral functions in equilibrium are related by the fluctuation-dissipation relation, their relation in nonequilibrium is only fixed by the initial condition or by the balance between the external driving and the dissipation. In nonequilibrium DMFT, one thus has to solve a set of coupled equations for the lesser, retarded and greater Green's functions, known as the Kadanoff-Baym equations.

A challenge for the implementation of the contour equations is that typical applications require a high accuracy. For example, one often wants to characterize the relaxation and thermalization of some excited state that involves small differences between the time-dependent observable and an independently computed expectation value in thermal equilibrium. In order to achieve high accuracy, while keeping a relatively large time step, it is important to use a high-order scheme for the solution of the integral-differential equations.

While insights into the nonequilibrium DMFT formalism can be gained from the Falicov-Kimball model, which has the virtue of being exactly solvable within DMFT, generic models of correlated electron systems such as the Hubbard model and its multiorbital extensions require a numerical solution of the effective quantum impurity model. Various techniques familiar from the study of impurity problems in equilibrium have recently been adapted to nonequilibrium situations, and tested as impurity solvers for nonequilibrium DMFT. One of these techniques is the continuous-time Monte Carlo approach, which is the method of choice for most equilibrium applications. This method has the advantage that it is numerically exact and can cover the weak-, intermediate-, and strongcorrelation regimes. However, the implementation on the Keldysh contour leads to a severe sign problem, which effectively restricts the applicability of this technique to nonequilibrium situations where the interesting dynamics is very fast ( $\sim$ femtoseconds in the case of electron systems).

We have also discussed and illustrated the use of low-order weak-coupling and strong-coupling perturbation theories as impurity solvers for DMFT. These methods are computationally less demanding than the Monte Carlo approach, and give reliable results in the weak- and strong-coupling regimes. In the weak-coupling perturbative method, it is often better to use a diagrammatic expansion based on bare (rather than renormalized) propagators. While the approximation is not conserving, it can still accurately reproduce the dynamics in the weak-coupling regime. The strong-coupling perturbative method, on the other hand, involves renormalized propagators (and is hence conserving), but still shows good convergence with the order of the approximation. The perturbative solvers have been important in the study of phenomena that require simulation times $\sim 100 \mathrm{fs}$, as in relaxation processes in a photodoped Mott insulator, or the calculation of the evolution of order parameters in symmetry-broken states. While the lowest-order implementations of the perturbative solvers are similar, in terms of computational effort, to the solution of the contour equations, the effort increases polynomially with the order of the approximation.

The impurity solvers described in this review have led to interesting insights into the nonequilibrium dynamics of 
correlated systems as typically described by the Hubbard model in quite a wide range of applications, and have thus been essential for establishing the formalism as a viable tool for the description of electronic excitation and relaxation phenomena in strongly correlated systems. We have reviewed the major topics which have been successfully addressed with nonequilibrium DMFT over the past several years. These applications can be grouped into (i) simulations of phenomena occurring in correlated lattice systems driven by strong electric fields, and (ii) the study of relaxation phenomena after time-dependent parameter changes. The former are relevant to pump-probe experiments on correlated electron systems, while the latter is more directly related to cold-atom systems, where parameters such as the depth of the lattice potential or the interaction strength can actually be varied. Both types of applications demonstrate that nonequilibrium DMFT calculations not only reproduce phenomena seen in experiments but also theoretically expected ones, such as collapse-and-revival oscillations after a quench into the strong-coupling regime (Eckstein, Kollar, and Werner, 2009), or Bloch oscillations in metallic systems subject to a strong dc field (Freericks, Turkowski, and Zlatić, 2006). More importantly, these calculations gave new insights into phenomena which emerge specifically in nonequilibrium correlated electron systems. Prime examples are the numerical demonstration of a dynamical phase transition in the relaxation dynamics after an interaction quench (Eckstein, Kollar, and Werner, 2009), or the finding that the effective Coulomb interaction can be tuned and even become attractive by the application of periodic electric fields (Tsuji et al., 2011).

The technical challenge in coming years will be to develop powerful and flexible impurity solvers for nonequilibrium DMFT. Besides the extension to multiorbital systems, an important direction for the future will be the study of electron-phonon coupled systems (Werner and Eckstein, 2013). A remarkable experimental result has recently been obtained by coherently exciting phonons in cuprates (Fausti et al., 2011), and nonequilibrium DMFT may provide insights into such phenomena. Cluster extensions of DMFT (Tsuji et al., 2013) will enable the study of the dynamics of $d$-wave superconductors. The extension of the DMFT formalism to spatially inhomogeneous systems will enable us to simulate nonequilibrium phenomena around surfaces or interfaces (Eckstein and Werner, 2013b). We also mention that equilibrium DMFT has recently been applied with remarkable success to bosonic systems (Anders et al., 2010, 2011), so that it will be desirable to extend it to a nonequilibrium bosonic DMFT (with bosonic impurity solvers).

For realistic materials calculations, the combination of electronic-structure input with DMFT is becoming an established and powerful method (Kotliar et al., 2006; Held, 2007). Thus a desirable direction is to combine the nonequilibrium DMFT formalism with the first-principles electronic structure, which will enable us to quantitatively analyze, e.g., timeresolved photoemission spectra. This step raises intriguing and fundamental questions, e.g., how the downfolding of the electronic structure into an effective lattice models should be done in nonequilibrium, and how one can treat electromagnetic fields far beyond linear response.
Finally, we emphasize that the study of nonequilibrium quantum systems has a long and very interdisciplinary history. Many important concepts were indeed developed in parallel in condensed-matter physics and field theory, and some of them were put forward at the emerging stage of quantum mechanics. (i) For instance, the application of intense lasers may drive a nonequilibrium phase transition of the vacuum (in the field-theoretic language) (Itakura et al., 2011), and this is intimately related to the photoinduced phase transitions discussed here, although there are orders of magnitude differences in the relevant energy scales. (ii) The dielectric breakdown of the Mott insulator is related to the Schwinger mechanism for the breakdown of the QED vacuum in high-energy physics (Heisenberg and Euler, 1936; Schwinger, 1951). The latter refers to a quantum tunneling across the mass gap of the electron (the energy required to create an electron-positron pair), which is $\Delta=2 m_{e} c^{2} \sim$ $10^{6} \mathrm{eV}$ with $m_{e}=5 \times 10^{5} \mathrm{eV}$ the electron mass and $c$ the speed of light. The threshold field strength $F_{\text {th }}$, at which $\xi e F_{\text {th }} \sim \Delta$, with $\xi$ the size of an electron-positron pair $\sim$ Compton wavelength $\hbar / m_{e} c$, is given in QED by $F_{\text {th }}^{\mathrm{QED}}=m_{e}^{2} c^{3} / e \hbar \sim 10^{8} \mathrm{~V} / \AA$. This is gigantic, although the possibility of realizing it with free-electron lasers is being discussed, while in condensed-matter physics, the energy gap $\Delta \sim 1 \mathrm{eV}$ is orders of magnitude smaller. In strongly correlated systems, where the gap is a many-body (Mott) gap $\Delta_{\text {Mott }}$, a Mott insulator in an intense electric field is predicted to become metallic with the threshold field (Oka and Aoki, 2010; Oka, 2012) $E_{\mathrm{th}}^{\mathrm{Mott}} \sim \Delta_{\text {Mott }} / \xi \sim 0.1 \mathrm{~V} / \AA$, where $\xi \sim$ $10 \AA$ in this case is the size of a doublon-hole pair for a typical Mott insulator with doublon-hole recombination corresponding to pair annihilation in field theory. The maximum intensity of ultrashort laser pulses currently available is well above this condensed-matter version of the Schwinger limit. (iii) The Floquet picture described in the present review also has a field-theoretic counterpart in the Furry picture. (iv) The interdisciplinary concepts extend to the relaxation processes. In fact, the concept of prethermalization (Berges, Borsányi, and Wetterich, 2004) was originally proposed in the study of the quark-gluon plasma production in hadrons out of equilibrium, as typically realized experimentally in the relativistic heavy-ion colliders (RHIC). Another example is the Kibble-Zurek mechanism (Kibble, 1976; Zurek, 1985) originally proposed for phase transitions in the early universe, which is now being studied in connection with quench dynamics near quantum critical points (Dziarmaga, Laguna, and Zurek, 1999), in cold-atom systems (Sadler et al., 2006; Saito, Kawaguchi, and Ueda, 2007; Weiler et al., 2008; Horiguchi, Oka, and Aoki, 2009), and even in real materials (Griffin et al., 2012).

\section{ACKNOWLEDGMENTS}

We thank R. Arita, P. Barmettler, P. Beaud, J. Berges, S. Biermann, A. Cavalleri, E. Demler, A. Dirks, N. Eurich, J. Freericks, L. Fu, A. Georges, D. Greif, E. Gull, H. Hafermann, C. Hauri, G. Ingold, S. Ishihara, C. Jung, S. Johnson, S. Koshihara, A. Lichtenstein, S. Kaiser, S. Kehrein, H. Kishida, T. Kitagawa, M. Knap, A. Komnik, N. Konno, H. Matsueda, 
A. Millis, S. Miyashita, M. Moeckel, L. Mühlbacher, Y. Murakami, S. Nakamura, K. Nasu, D. Nicoletti, T. Ogawa, H. Okamoto, O. Parcollet, V. Pietilä, R. Shimano, K. Tanaka, L. Tarruell, T. Tohyama, S. Tsuneyuki, K. Ueda, D. Vollhardt, and $\mathrm{K}$. Yonemitsu for stimulating discussions and collaborations on topics related to this review. H. A., T. O., and N. T. were supported in part by Grants-in-Aid for Scientific Research on Innovative Areas from JSPS, Grants No. 23740260, No. 23104709, and No. 23110707. M. K. was supported in part by Transregio 80 of the Deutsche Forschungsgemeinschaft. M. E., N. T., and P. W. acknowledge support by the Swiss National Science Foundation (Grant No. PP0022-118866) and FP7/ERC starting grant No. 278023.

\section{Appendix A: Numerical Solution of Volterra Integral-differential Equation}

In this Appendix, we briefly discuss the numerical implementation of the Volterra integral-differential equation

$$
\frac{d}{d t} y(t)=q(t)+p(t) y(t)+\int_{0}^{t} d \bar{t} k(t, \bar{t}) y(\bar{t})
$$

This type of equation frequently appears in nonequilibrium DMFT calculations [Sec. II.B, Eq. (43)], in particular in solving the nonequilibrium Dyson equation (Sec. II.A.1.d). One also encounters a Volterra integral equation of the form

$$
y(t)=q(t)+\int_{0}^{t} d \bar{t} k(t, \bar{t}) y(\bar{t})
$$

which is a special case of Eq. (A1), and can be solved in the same way as Eq. (A1).

Various numerical algorithms to solve Eq. (A1) are found in the literature (Linz, 1985; Brunner and van der Houwen, 1986; Press et al., 1992). Here we present the implicit Runge-Kutta method (or the collocation method) (Tsuji and Werner, 2013), which may not be the most efficient one, but it allows us to discuss the relevant issues. In practice, we discretize the time with equal spacing, $t_{i}=i \times \Delta t(i=0,1, \ldots, n)$, with $\Delta t=t_{\max } / n$. It is crucial to employ higher-order schemes to accurately simulate the long-time evolution. The $m$ th order scheme has numerical errors of $O\left(n(\Delta t)^{m+1}\right)=$ $O\left(t_{\max }(\Delta t)^{m}\right)$. Typically we require $m \geq 2$ to control the errors. In the following, we explicitly give expressions for the second- and fourth-order schemes.

Equation (A1) can be solved by increasing $t_{\max }=t_{n}$ step by step on the discretized grid from the initial condition $y\left(t_{0}\right)=$ $y(0)$ due to the causality. To get $y\left(t_{n}\right)$, we replace the differential operator on the left-hand side of Eq. (A1) by an integral, which is numerically evaluated by an appropriate numerical integration formula

$$
y\left(t_{n}\right)-y\left(t_{0}\right)=\int_{t_{0}}^{t_{n}} d \bar{t} y^{\prime}(\bar{t}) \approx \Delta t \sum_{i=0}^{n} w_{n, i} y^{\prime}\left(t_{i}\right),
$$

with $w_{n, i}(i=0,1,2, \ldots, n)$ the corresponding weights. Since $y\left(t_{n-1}\right)$ is already known from the previous calculation, we also use

$y\left(t_{n-1}\right)-y\left(t_{0}\right)=\int_{t_{0}}^{t_{n-1}} d \bar{t} y^{\prime}(\bar{t}) \approx \Delta t \sum_{i=0}^{n-1} w_{n-1, i} y^{\prime}\left(t_{i}\right)$.

By subtracting Eq. (A4) from Eq. (A3), we get

$y\left(t_{n}\right)-y\left(t_{n-1}\right)=\Delta t \sum_{i=0}^{n-1}\left(w_{n, i}-w_{n-1, i}\right) y^{\prime}\left(t_{i}\right)+\Delta t w_{n, n} y^{\prime}\left(t_{n}\right)$.

Here $y^{\prime}\left(t_{n}\right)$ is evaluated from Eq. (A1) as

$$
\begin{aligned}
y^{\prime}\left(t_{n}\right) & =q\left(t_{n}\right)+p\left(t_{n}\right) y\left(t_{n}\right)+\int_{0}^{t_{n}} d \bar{t} k\left(t_{n}, \bar{t}\right) y(\bar{t}) \\
& \approx q\left(t_{n}\right)+p\left(t_{n}\right) y\left(t_{n}\right)+\Delta t \sum_{i=0}^{n} w_{n, i} k\left(t_{n}, t_{i}\right) y\left(t_{i}\right) .
\end{aligned}
$$

Equations (A5) and (A6) consist of a set of linear equations for $y\left(t_{n}\right)$, so that one can explicitly solve them,

$$
\begin{aligned}
y\left(t_{n}\right)= & {\left[1-\Delta t w_{n, n} p\left(t_{n}\right)-\left(\Delta t w_{n, n}\right)^{2} k\left(t_{n}, t_{n}\right)\right]^{-1} } \\
& \times\left\{y\left(t_{n-1}\right)+\Delta t \sum_{i=0}^{n-1}\left(w_{n, i}-w_{n-1, i}\right) y^{\prime}\left(t_{i}\right)\right. \\
& \left.+\Delta t w_{n, n}\left[q\left(t_{n}\right)+\Delta t \sum_{i=0}^{n-1} w_{n, i} k\left(t_{n}, t_{i}\right) y\left(t_{i}\right)\right]\right\} .
\end{aligned}
$$

As we will see below, $w_{n, i}-w_{n-1, i}$ vanishes for most $i$ 's, so that one has to store $y^{\prime}\left(t_{i}\right)$ for only a few $i(i=n-1$ in the second-order scheme, and $i=n-3, n-2, n-1$ in the fourth-order scheme). For the use in the next steps $\left(t_{\max }=t_{n+1}, \ldots\right)$, we calculate $y^{\prime}\left(t_{n}\right)$ from Eq. (A6) with $y\left(t_{n}\right)$ substituted with the result of Eq. (A7). To avoid repeated calculations of the sum in Eqs. (A6) and (A7), it is efficient to store them in memory.

In the same way, the Volterra integral equation (A2) is solved as

$$
\begin{aligned}
y\left(t_{n}\right)= & {\left[1-\Delta t w_{n, n} k\left(t_{n}, t_{n}\right)\right]^{-1} } \\
& \times\left[q\left(t_{n}\right)+\Delta t \sum_{i=0}^{n-1} w_{n, i} k\left(t_{n}, t_{i}\right) y\left(t_{i}\right)\right] .
\end{aligned}
$$

In the $m$ th order scheme, we employ the numerical integration formula with numerical errors of $O\left(n(\Delta t)^{m+1}\right)=$ $O\left(t_{\max }(\Delta t)^{m}\right)$. In the second-order scheme, one can use the trapezoid rule with weights

$$
w_{n, i}= \begin{cases}1 / 2 & i=0, n \\ 1 & 1 \leq i \leq n-1\end{cases}
$$

In the fourth-order scheme, one can use Simpson's rule for $n=2$, 


$$
w_{2, i}= \begin{cases}1 / 3 & i=0,2, \\ 4 / 3 & i=1,\end{cases}
$$

Simpson's $3 / 8$ rule for $n=3$

$$
w_{3, i}= \begin{cases}3 / 8 & i=0,3 \\ 9 / 8 & i=1,2\end{cases}
$$

the composite Simpson's rule for $n=4$

$$
w_{4, i}= \begin{cases}1 / 3 & i=0,4, \\ 4 / 3 & i=1,3, \\ 2 / 3 & i=2,\end{cases}
$$

and the fourth-order Gregory's rule for $n \geq 5$

$$
w_{n, i}= \begin{cases}3 / 8 & i=0, n, \\ 7 / 6 & i=1, n-1, \\ 23 / 24 & i=2, n-2, \\ 1 & 3 \leq i \leq n-3 .\end{cases}
$$

The remaining task is to get the starting value $y\left(t_{1}\right)$. Since the higher-order integral formulas need at least three points, the above approach cannot be directly applied for $n=1$. One way to get around this is to take very fine grids on $t_{0} \leq t \leq t_{1}$, and use a lower-order integral formula (trapezoid rule). Another way is to take the middle point $t_{1 / 2}=\Delta t / 2$ (Linz, 1985), and apply Simpson's rule to the integral from $t_{0}$ to $t_{1}$ :

$$
y\left(t_{1}\right)-y\left(t_{0}\right) \approx \frac{\Delta t}{6}\left[y^{\prime}\left(t_{0}\right)+4 y^{\prime}\left(t_{1 / 2}\right)+y^{\prime}\left(t_{1}\right)\right] .
$$

The value at the middle point is obtained from the quadratic interpolation

$$
y^{\prime}\left(t_{1 / 2}\right) \approx \frac{3}{8} y^{\prime}\left(t_{0}\right)+\frac{3}{4} y^{\prime}\left(t_{1}\right)-\frac{1}{8} y^{\prime}\left(t_{2}\right),
$$

which has an error of $O\left((\Delta t)^{3}\right)$ for the smooth function $y(t)$. Since $y^{\prime}\left(t_{1 / 2}\right)$ is multiplied with $\Delta t$ in Eq. (A14), the overall error is of $O\left((\Delta t)^{4}\right)$, which is compatible with that for the fourth-order scheme. $y^{\prime}\left(t_{0}\right)$ is known from the initial condition. $y^{\prime}\left(t_{2}\right)$ is derived from Eq. (A6). $y^{\prime}\left(t_{1}\right)$ is calculated by Simpson's rule with the middle point

$$
\begin{aligned}
y^{\prime}\left(t_{1}\right) \approx & q\left(t_{1}\right)+p\left(t_{1}\right) y\left(t_{1}\right)+\frac{\Delta t}{6}\left[k\left(t_{1}, t_{0}\right) y\left(t_{0}\right)\right. \\
& \left.+4 k\left(t_{1}, t_{1 / 2}\right) y\left(t_{1 / 2}\right)+k\left(t_{1}, t_{1}\right) y\left(t_{1}\right)\right] .
\end{aligned}
$$

One can repeat the quadratic interpolation to get the middlepoint values

$$
\begin{gathered}
y\left(t_{1 / 2}\right) \approx \frac{3}{8} y\left(t_{0}\right)+\frac{3}{4} y\left(t_{1}\right)-\frac{1}{8} y\left(t_{2}\right), \\
k\left(t_{1}, t_{1 / 2}\right) \approx \frac{3}{8} k\left(t_{1}, t_{0}\right)+\frac{3}{4} k\left(t_{1}, t_{1}\right)-\frac{1}{8} k\left(t_{1}, t_{2}\right) .
\end{gathered}
$$

Thus, the equations for $y\left(t_{1}\right)$ depend on $y\left(t_{2}\right)$. On the other hand, $y\left(t_{2}\right)$ can be determined from $y\left(t_{0}\right)$ and $y\left(t_{1}\right)$ as described above. In total, we have a combined set of linear equations that determines $y\left(t_{1}\right)$ and $y\left(t_{2}\right)$ simultaneously.

The middle-point approach has a subtle problem when it is applied to a retarded kernel that has a causality, $k\left(t, t^{\prime}\right)=0$ $\left(t<t^{\prime}\right)$. Since $k\left(t, t^{\prime}\right)$ is smooth only for $t \geq t^{\prime}$, the quadratic interpolation (A18) using $k\left(t_{1}, t_{2}\right)\left(t_{1}<t_{2}\right)$ is inapplicable in the present form. This problem can be avoided by taking the mirror image for $k\left(t, t^{\prime}\right)$ in $t<t^{\prime}$ to realize a function that is smooth in the entire $\left(t, t^{\prime}\right)$ and is equal to $k\left(t, t^{\prime}\right)$ for $t>t^{\prime}$ (Tsuji and Werner, 2013).

\section{Appendix B: Sample Programs}

For a pedagogical purpose, we provide sample program codes for the nonequilibrium DMFT, in both $\mathrm{C}++$ and FORTRAN, as Supplemental Material [363]. To enhance the readability of the codes, we focus on a particular setup: the program solves an interaction-quench problem (Sec. III.B.1) for the single-band Hubbard model (60) with the semicircular density of states at half filling. It assumes a paramagnetic phase with no long-range orders. The impurity solver is the second-order weak-coupling perturbation theory (iterated perturbation theory) (Sec. II.C.4), where the self-energy is given by

$$
\Sigma\left(t, t^{\prime}\right)=U(t) U\left(t^{\prime}\right) \mathcal{G}_{0}\left(t, t^{\prime}\right) \mathcal{G}_{0}\left(t^{\prime}, t\right) \mathcal{G}_{0}\left(t, t^{\prime}\right)
$$

with $\mathcal{G}_{0}\left(t, t^{\prime}\right)$ the Weiss Green's function. To solve the Dyson equation (Sec. II.A.1.d), we use the second-order scheme (Appendix A) for the Volterra integral-differential equation.

To install the codes, download the tar file from the Supplemental Material [363] and untar them in a certain working directory:

\section{\$ tar zxvf noneq-dmft.tar.gz}

It generates the subdirectories cxx and fortran, which contain the $\mathrm{C}++$ and FORTRAN codes, respectively. To compile them, one needs the FFTW library for fast Fourier transformation, which can be downloaded. ${ }^{1}$ One should specify the path for the FFTW library in the make file, which is by default set to /usr/local. To build the code, execute make in the directory in which it is installed:

$\$$ make

If the build is successful, it generates an executable file a.out. It requires input parameters, which are listed in the file parm.sh. In the sample programs, the parameters dos (density of states) and solver (impurity solver) are restricted to be semicircular and IPT (iterative perturbation theory), respectively, while the other parameters can be freely changed. After choosing the parameters, run the program by typing:

\footnotetext{
${ }^{1}$ See http://www.fftw.org.
} 


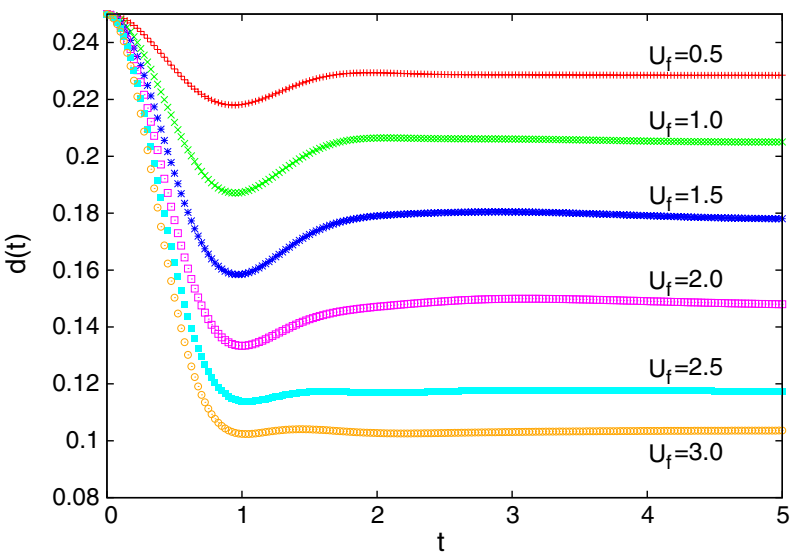

FIG. 32 (color online). The results of the sample program for the time evolution of the double occupancy $d(t)$ after interaction quenches from $U_{i}=0$ to $U_{f}$ with $\beta=16$.

\section{\$./parm.sh}

During the execution, it outputs a measure of the DMFT convergence (IG0_new-G0_oldl) as well as the time up to which the system has been evolved. After the simulation has finished, it automatically creates the following output files:

\section{density}

double-occupancy

interaction-energy

kinetic-energy

total-energy

In Fig. 32, we show some results obtained with the sample program. These results can be used to check the correctness of the program output.

The sample programs have been designed and implemented by $\mathrm{N}$. Tsuji, one of the authors. The codes can be used and modified for noncommercial purposes, but their use must be acknowledged in publications with a citation to this review article.

\section{REFERENCES}

Abrikosov, A. A, L. P. Gorkov, and I. E. Dzyaloshinski, 1975, Methods of Quantum Field Theory in Statistical Physics (Dover, New York).

Ajisaka, S., H. Nishimura, S. Tasaki, and I. Terasaki, 2009, Prog. Theor. Phys. 121, 1289.

Allen, P. B., 1987, Phys. Rev. Lett. 59, 1460.

Althorpe, S. C., D. J. Kouri, D. K. Hoffman, and N. Moiseyev, 1997, Chem. Phys. 217, 289.

Altshuler, B. L., V. E. Kravtsov, I. V. Lerner, and I. L. Aleiner, 2009, Phys. Rev. Lett. 102, 176803.

Amaricci, A., C. Weber, M. Capone, and G. Kotliar, 2012, Phys. Rev. B 86, 085110.
Anders, P., E. Gull, L. Pollet, M. Troyer, and P. Werner, 2010, Phys. Rev. Lett. 105, 096402.

Anders, P., E. Gull, L. Pollet, M. Troyer, and P. Werner, 2011, New J. Phys. 13, 075013.

André, P., M. Schiró, and M. Fabrizio, 2012, Phys. Rev. B 85, 205118.

Aron, C., 2012, Phys. Rev. B 86, 085127.

Aron, C., G. Kotliar, and C. Weber, 2012, Phys. Rev. Lett. 108, 086401.

Aron, C., C. Weber, and G. Kotliar, 2013, Phys. Rev. B 87, 125113.

Arrigoni, E., M. Knap, and W. von der Linden, 2013, Phys. Rev. Lett. 110, 086403

Asamitsu, A., Y. Tomioka, H. Kuwahara, and Y. Tokura, 1997, Nature (London) 388, 50.

Assaad, F. F., and T. C. Lang, 2007, Phys. Rev. B 76, 035116.

Avron, J.E., and A. Elgart, 1999, Commun. Math. Phys. 203, 445.

Balzer, K., and M. Bonitz, 2013, Nonequilibrium Green's Functions Approach to Inhomogeneous Systems, Lecture Notes in Physics Vol. 867 (Spinger, Heidelberg, Germany).

Balzer, M., N. Gdaniec, and M. Potthoff, 2012, J. Phys. Condens. Matter 24, 035603.

Balzer, M., and M. Potthoff, 2011, Phys. Rev. B 83, 195132.

Barankov, R. A., and L.S. Levitov, 2006, Phys. Rev. Lett. 96, 230403.

Barmettler, P., M. Punk, V. Gritsev, E. Demler, and E. Altman, 2009, Phys. Rev. Lett. 102, 130603.

Barnes, S. E., 1976, J. Phys. F 6, 1375.

Baym, G., 1962, Phys. Rev. 127, 1391.

Baym, G., and L. P. Kadanoff, 1961, Phys. Rev. 124, 287.

Beaurepaire, E., J.-C. Merle, A. Daunois, and J.-Y. Bigot, 1996, Phys. Rev. Lett. 76, 4250.

Ben Dahan, M., E. Peik, J. Reichel, Y. Castin, and C. Salomon, 1996, Phys. Rev. Lett. 76, 4508.

Berges, J., S. Borsányi, and C. Wetterich, 2004, Phys. Rev. Lett. 93, 142002.

Berges, J., A. Rothkopf, and J. Schmidt, 2008, Phys. Rev. Lett. 101, 041603.

Bertoncini, R., and A. P. Jauho, 1991, Phys. Rev. B 44, 3655.

Bickers, N. E., 1987, Rev. Mod. Phys. 59, 845.

Bickers, N. E., D. L. Cox, and J. W. Wilkins, 1987, Phys. Rev. B 36, 2036.

Bloch, F., 1929, Z. Phys. 52, 555.

Bloch, I., J. Dalibard, and W. Zwerger, 2008, Rev. Mod. Phys. 80, 885.

Bonitz, M., 2000, Progress in Nonequilibrium Green's Functions (World Scientific, Singapore).

Bonitz, M., and K. Balzer, 2010, Progress in Nonequilibrium Green's Functions IV, Journal of Physics Conference Series Vol. 220 (World Scientific, Singapore), pp. 011001-012023.

Bonitz, M., and A. Filinov, 2006, Progress in Nonequilibrium Green's Functions III, Journal of Physics Conference Series Vol. 35 (World Scientific, Singapore), pp. 001-042.

Bonitz, M., and D. Semkat, 2003, Progress in Nonequilibrium Green's Functions II (World Scientific, Singapore).

Born, M., and V. A. Fock, 1928, Z. Phys. A 51, 165.

Boulware, D. G., 1966, Phys. Rev. 151, 1024.

Brandes, T., and J. Robinson, 2002, Phys. Status Solidi B 234, 378.

Brandt, U., and C. Mielsch, 1989, Z. Phys. B 75, 365.

Brandt, U., and C. Mielsch, 1990, Z. Phys. B 79, 295.

Brandt, U., and C. Mielsch, 1991, Z. Phys. B 82, 37.

Braun, S., J. P. Ronzheimer, M. Schreiber, S. S. Hodgman, T. Rom, I. Bloch, and U. Schneider, 2013, Science 339, 52. 
Breuer, H.-P., and F. Petruccione, 2002, The Theory of Open Quantum Systems (Oxford University Press, Oxford).

Brout, R., 1960, Phys. Rev. 118, 1009.

Brunner, H., and P. J. van der Houwen, 1986, The Numerical Solution of Volterra Equations (North-Holland, Amsterdam).

Bulla, R., T. A. Costi, and T. Pruschke, 2008, Rev. Mod. Phys. 80, 395.

Büttiker, M., 1985, Phys. Rev. B 32, 1846.

Büttiker, M., 1986, Phys. Rev. B 33, 3020.

Calabrese, P., and J. Cardy, 2006, Phys. Rev. Lett. 96, 136801.

Caldeira, A. O., and A. J. Leggett, 1981, Phys. Rev. Lett. 46, 211.

Cassidy, A. C., C. W. Clark, and M. Rigol, 2011, Phys. Rev. Lett. 106, 140405.

Caux, J.-S., and J. Mossel, 2011, J. Stat. Mech. P02023.

Caviglia, A. D. et al., 2012, Phys. Rev. Lett. 108, 136801.

Cazalilla, M. A., 2006, Phys. Rev. Lett. 97, 156403.

Cohen, G., E. Gull, D. R. Reichman, A. J. Millis, and E. Rabani, 2013, Phys. Rev. B 87, 195108.

Cohen, G., and E. Rabani, 2011, Phys. Rev. B 84, 075150.

Coleman, P., 1984, Phys. Rev. B 29, 3035.

Coleman, P., 2007, in Handbook of Magnetism and Advanced Magnetic Materials, edited by H. Kronmüller and S. Parkin, Vol. 1 (John Wiley and Sons, New York).

Creffield, C.E., and T. S. Monteiro, 2006, Phys. Rev. Lett. 96, 210403.

Dal Conte, S., et al., 2012, Science 335, 1600.

Daley, A., C. Kollath, U. Schollwock, and G. Vidal, 2004, J. Stat. Mech., P04005.

Danielewicz, P., 1984a, Ann. Phys. (N.Y.) 152, 239.

Danielewicz, P., 1984b, Ann. Phys. (N.Y.) 152, 305.

Davies, J. H., and J. W. Wilkins, 1988, Phys. Rev. B 38, 1667.

De Leo, L., M. Civelli, and G. Kotliar, 2008, Phys. Rev. B 77, 075107.

Deutsch, J. M., 1991, Phys. Rev. A 43, 2046.

Dittrich, T., P. Hänggi, G. L. Ingold, B. Kramer, G. Schön, and W. Zwerger, 1998, Quantum Transport and Dissipation (Wiley-VCH, Weinheim).

Dunlap, D. H., and V. M. Kenkre, 1986, Phys. Rev. B 34, 3625.

Dziarmaga, J., 2010, Adv. Phys. 59, 1063.

Dziarmaga, J., P. Laguna, and W. H. Zurek, 1999, Phys. Rev. Lett. 82, 4749.

Eckardt, A., C. Weiss, and M. Holthaus, 2005, Phys. Rev. Lett. 95, 260404.

Eckstein, M., A. Hackl, S. Kehrein, M. Kollar, M. Moeckel, P. Werner, and F. A. Wolf, 2009, Eur. Phys. J. Spec. Top. 180, 217.

Eckstein, M., and M. Kollar, 2008a, Phys. Rev. B 78, 245113.

Eckstein, M., and M. Kollar, 2008b, Phys. Rev. Lett. 100, 120404.

Eckstein, M., and M. Kollar, 2008c, Phys. Rev. B 78, 205119.

Eckstein, M., and M. Kollar, 2010, New J. Phys. 12, 055012.

Eckstein, M., M. Kollar, and P. Werner, 2009, Phys. Rev. Lett. 103, 056403.

Eckstein, M., M. Kollar, and P. Werner, 2010, Phys. Rev. B 81, 115131.

Eckstein, M., T. Oka, and P. Werner, 2010, Phys. Rev. Lett. 105, 146404.

Eckstein, M., and P. Werner, 2010, Phys. Rev. B 82, 115115.

Eckstein, M., and P. Werner, 2011a, Phys. Rev. Lett. 107, 186406.

Eckstein, M., and P. Werner, 2011b, Phys. Rev. B 84, 035122.

Eckstein, M., and P. Werner, 2013a, J. Phys. Conf. Ser. 427, 012005.

Eckstein, M., and P. Werner, 2013b, Phys. Rev. B 88, 075135.

Eckstein, M., and P. Werner, 2013c, Phys. Rev. Lett. 110, 126401.

Ehrke, H., et al., 2011, Phys. Rev. Lett. 106, 217401.
Eichberger, M., H. Schäfer, M. Krumova, M. Beyer, J. Demsar, H. Berger, G. Moriena, G. Sciaini, and R. J. D. Miller, 2010, Nature (London) 468, 799.

Eurich, N., M. Eckstein, and P. Werner, 2011, Phys. Rev. B 83, 155122.

Faisal, F. H. M., 1989, Comput. Phys. Rep. 9, 57.

Falicov, L. M., and J. C. Kimball, 1969, Phys. Rev. Lett. 22, 997.

Fausti, D., R. I. Tobey, N. Dean, S. Kaiser, A. Dienst, M. C. Hoffmann, S. Pyon, T. Takayama, H. Takagi, and A. Cavalleri, 2011, Science 331, 189.

Feldman, D. E., 2005, Phys. Rev. Lett. 95, 177201.

Fetter, A. L., and J. D. Walecka, 2003, Quantum Theory of ManyParticle Systems (Dover, New York).

Feynman, R. P., and J. F. L. Vernon, 1963, Ann. Phys. (N.Y.) 24, 118. Floquet, G., 1883, Ann. Sci. Ec. Normale Super. 12, 47.

Foreman, B. A., 2002, Phys. Rev. B 66, 165212.

Freericks, J. K., 1994, Phys. Rev. B 50, 403.

Freericks, J. K., 2004, Phys. Rev. B 70, 195342.

Freericks, J. K., 2008, Phys. Rev. B 77, 075109.

Freericks, J. K., and M. Jarrell, 1994, Phys. Rev. B 50, 6939.

Freericks, J. K., and A. V. Joura, 2008, in Electron Transport in Nanosystems, edited by J. Bonča and S. Kruchinin (Springer, Berlin), p. 219.

Freericks, J. K., H. R. Krishnamurthy, and T. Pruschke, 2009, Phys. Rev. Lett. 102, 136401.

Freericks, J. K., V. M. Turkowski, and V. Zlatić, 2006, Phys. Rev. Lett. 97, 266408.

Freericks, J. K., and V. Zlatić, 2003, Rev. Mod. Phys. 75, 1333.

Fujii, T., and K. Ueda, 2003, Phys. Rev. B 68, 155310.

Gambassi, A., and P. Calabrese, 2011, Europhys. Lett. 95, 66007.

Gell-Mann, M., and F. Low, 1951, Phys. Rev. 84, 350.

Georges, A., and G. Kotliar, 1992, Phys. Rev. B 45, 6479.

Georges, A., G. Kotliar, W. Krauth, and M. J. Rozenberg, 1996, Rev. Mod. Phys. 68, 13.

Glück, M., A. R. Kolovsky, and H. J. Korsch, 2002, Phys. Rep. 366, 103.

Golež, D., J. Bonča, M. Mierzejewski, and L. Vidmar, 2013, arXiv:1311.5574v1.

Gramsch, C., K. Balzer, M. Eckstein, and M. Kollar, 2013, arXiv:1306.6315.

Grandi, C. D., and A. Polkovnikov, 2010, in Quantum Quenching, Annealing and Computation, edited by A. Chandra, Anjan Kumar amd Das, and B. K. Chakrabarti, Lecture Notes in Physics Vol. 802 (Springer, New York), p. 75.

Greiner, M., O. Mandel, T. W. Hänsch, and I. Bloch, 2002, Nature (London) 419, 51.

Grewe, N., and H. Keiter, 1981, Phys. Rev. B 24, 4420.

Griffin, S. M., M. Lilienblum, K. T. Delaney, Y. Kumagai, M. Fiebig, and N. A. Spaldin, 2012, Phys. Rev. X 2, 041022.

Grifoni, M., and P. Hänggi, 1998, Phys. Rep. 304, 229.

Gros, C., and R. Valentí, 1993, Phys. Rev. B 48, 418.

Grossmann, F., T. Dittrich, P. Jung, and P. Hänggi, 1991, Phys. Rev. Lett. 67, 516.

Grossmann, F., and P. Hänggi, 1992, Europhys. Lett. 18, 571.

Guiot, V., L. Cario, E. Janod, B. Corraze, V. Ta Phuoc, M. Rozenberg, P. Stoliar, T. Cren, and D. Roditchev, 2013, Nat. Commun. 4, 1722.

Gull, E., A. J. Millis, A. I. Lichtenstein, A. N. Rubtsov, M. Troyer, and P. Werner, 2011, Rev. Mod. Phys. 83, 349.

Gull, E., D. R. Reichman, and A. J. Millis, 2010, Phys. Rev. B 82, 075109 . 
Gull, E., D. R. Reichman, and A. J. Millis, 2011, Phys. Rev. B 84, 085134.

Gull, E., P. Werner, O. Parcollet, and M. Troyer, 2008, Europhys. Lett. 82, 57003.

Hafermann, H., C. Jung, S. Brener, M. I. Katsnelson, A. N. Rubtsov, and A. I. Lichtenstein, 2009, Europhys. Lett. 85, 27007.

Hamerla, S. A., and G. Uhrig, 2013, Phys. Rev. B 87, 064304.

Han, J. E., 2013, Phys. Rev. B 87, 085119.

Han, J. E., and R. J. Heary, 2007, Phys. Rev. Lett. 99, 236808.

Haque, M., and F. E. Zimmer, 2013, Phys. Rev. A 87, 033613.

Hastings, W. K., 1970, Biometrika 57, 97.

Haug, H., and A.-P. Jauho, 2008, Quantum Kinetics in Transport and Optics of Semiconductors (Springer, Berlin), 2nd ed.

Haule, K., S. Kirchner, J. Kroha, and P. Wölfle, 2001, Phys. Rev. B 64, 155111

Heary, R. J., and J.E. Han, 2009, Phys. Rev. B 80, 035102.

Hedin, L., and J. Lee, 2002, J. Electron Spectrosc. Relat. Phenom. 124, 289

Heidrich-Meisner, F., I. González, K. A. Al-Hassanieh, A. E. Feiguin, M. J. Rozenberg, and E. Dagotto, 2010, Phys. Rev. B 82, 205110

Heisenberg, W., and H. Euler, 1936, Z. Phys. 98, 714, its English translation may be found in arXiv:physics/0605038.

Held, K., 2007, Adv. Phys. 56, 829.

Hellmann, S., et al., 2010, Phys. Rev. Lett. 105, 187401.

Hershfield, S., 1993, Phys. Rev. Lett. 70, 2134.

Hershfield, S., J. H. Davies, and J. W. Wilkins, 1991, Phys. Rev. Lett. 67, 3720 .

Hershfield, S., J. H. Davies, and J. W. Wilkins, 1992, Phys. Rev. B 46, 7046.

Hettler, M. H., M. Mukherjee, M. Jarrell, and H. R. Krishnamurthy, 2000, Phys. Rev. B 61, 12739.

Hettler, M. H., A. N. Tahvildar-Zadeh, M. Jarrell, T. Pruschke, and H. R. Krishnamurthy, 1998, Phys. Rev. B 58, R7475.

Heyl, M., A. Polkovnikov, and S. Kehrein, 2013, Phys. Rev. Lett. 110, 135704

Hill, G. W., 1886, Acta Math. 8, 1.

Hirori, H., A. Doi, F. Blanchard, and K. Tanaka, 2011, Appl. Phys. Lett. 98, 091106.

Hirori, H., K. Shinokita, M. Shirai, S. Tani, Y. Kadoya, and K. Tanaka, 2011, Nat. Commun. 2, 594.

Hofmann, F., M. Eckstein, E. Arrigoni, and M. Potthoff, 2013, Phys. Rev. B 88, 165124.

Hohenberg, P. C., and B. I. Halperin, 1977, Rev. Mod. Phys. 49, 435.

Holthaus, M., 1992, Phys. Rev. Lett. 69, 351.

Horiguchi, N., T. Oka, and H. Aoki, 2009, J. Phys. Conf. Ser. 150, 032007.

Ichikawa, H. et al., 2011, Nat. Mater. 10, 101.

Imada, M., A. Fujimori, and Y. Tokura, 1998, Rev. Mod. Phys. 70, 1039.

Inada, T. S., I. Terasaki, H. Mori, and T. Mori, 2009, Phys. Rev. B 79, 165102.

Itakura, K., S. Iso, and T. Takahashi, 2011, Eds., Proceedings of International Conference on Physics in Intense Fields (PIF2010) (KEK, Tsukuba).

Iwai, S., M. Ono, A. Maeda, H. Matsuzaki, H. Kishida, H. Okamoto, and Y. Tokura, 2003, Phys. Rev. Lett. 91, 057401.

Janiš, V., 1991, Z. Phys. B 83, 227.

Janiš, V., and D. Vollhardt, 1992, Int. J. Mod. Phys. B 06, 731.

Jarrell, M., 1992, Phys. Rev. Lett. 69, 168.

Jaynes, E. T., 1957a, Phys. Rev. 106, 620.

Jaynes, E. T., 1957b, Phys. Rev. 108, 171.

Jördens, R., N. Strohmaier, K. Günter, H. Moritz, and T. Esslinger, 2008, Nature (London) 455, 204.
Joura, A. V., J. K. Freericks, and T. Pruschke, 2008, Phys. Rev. Lett. 101, 196401.

Jung, C., A. Lieder, S. Brener, H. Hafermann, B. Baxevanis, A. Chudnovskiy, A. Rubtsov, M. Katsnelson, and A. Lichtenstein, 2012, Ann. Phys. (Berlin) 524, 49.

Kadanoff, L. P., and G. Baym, 1962, Quantum Statistical Mechanics (W. A. Benjamin, New York).

Kamenev, A., 2011, Field Theory of Non-Equilibrium Systems (Cambridge University Press, Cambridge, England).

Kanamori, Y., H. Matsueda, and S. Ishihara, 2009, Phys. Rev. Lett. 103, 267401

Kanamori, Y., H. Matsueda, and S. Ishihara, 2011, Phys. Rev. Lett. 107, 167403.

Karrasch, C., and D. Schuricht, 2013, Phys. Rev. B 87, 195104.

Kato, T., 1950, J. Phys. Soc. Jpn. 5, 435.

Keiter, H., and J. C. Kimball, 1971, Int. J. Magn. 1, 233.

Keldysh, L. V., 1964, Zh. Eksp. Teor. Fiz. 47, 1515 [Sov. Phys. JETP 20, 1018 (1965)].

Keller, M., W. Metzner, and U. Schollwöck, 2001, Phys. Rev. Lett. 86, 4612.

Kemper, A. F., M. Sentef, B. Moritz, C. C. Kao, Z. X. Shen, J. K. Freericks, and T. P. Devereaux, 2013, Phys. Rev. B 87, 235139.

Khurana, A., 1990, Phys. Rev. Lett. 64, 1990.

Kibble, T. W. B., 1976, J. Phys. A 9, 1387.

Kierig, E., U. Schnorrberger, A. Schietinger, J. Tomkovic, and M. K. Oberthaler, 2008, Phys. Rev. Lett. 100, 190405.

Kishida, H., H. Matsuzaki, H. Okamoto, T. Manabe, M. Yamashita, Y. Taguchi, and Y. Tokura, 2000, Nature (London) 405, 929.

Kitagawa, T., T. Oka, A. Brataas, L. Fu, and E. Demler, 2011, Phys. Rev. B 84, 235108.

Klein, M. J., 1956, Phys. Rev. 104, 589.

Knap, M., W. von der Linden, and E. Arrigoni, 2011, Phys. Rev. B 84, 115145 .

Koga, A., and P. Werner, 2011, Phys. Rev. A 84, 023638.

Köhler, H. S., N. H. Kwong, and H. A. Yousif, 1999, Comput. Phys. Commun. 123, 123.

Kohn, W., 1959, Phys. Rev. 115, 1460.

Kollar, M., F. A. Wolf, and M. Eckstein, 2011, Phys. Rev. B 84, 054304

Kollath, C., A. M. Läuchli, and E. Altman, 2007, Phys. Rev. Lett. 98, 180601.

Kotliar, G., S. Y. Savrasov, K. Haule, V. S. Oudovenko, O. Parcollet, and C. A. Marianetti, 2006, Rev. Mod. Phys. 78, 865.

Kotliar, G., S. Y. Savrasov, G. Pálsson, and G. Biroli, 2001, Phys. Rev. Lett. 87, 186401.

Kübler, C., H. Ehrke, R. Huber, R. Lopez, A. Halabica, R. F. Haglund, and A. Leitenstorfer, 2007, Phys. Rev. Lett. 99, 116401.

Kubo, R., 1957, J. Phys. Soc. Jpn. 12, 570.

Kuramoto, Y., 1983, Z. Phys. B 53, 37.

Landau, L. D., 1932, Phys. Z. Sowjetunion 2, 46.

Langreth, D. C., 1976, in Linear and Nonlinear Electron Transport in Solids, edited by J. T. Devreese and V. E. van Doren (Plenum Press, New York).

Larkin, A. I., and Y. N. Ovchinnikov, 1975, Zh. Eksp. Teor. Fiz. 68, 1915 [Sov. Phys. JETP 41, 960 (1975)].

Lenarčič, Z., and P. Prelovšek, 2012, Phys. Rev. Lett. 108, 196401.

Lenarčič, Z., and P. Prelovšek, 2013, Phys. Rev. Lett. 111, 016401. Lichtenstein, A. I., and M. I. Katsnelson, 2000, Phys. Rev. B 62, R9283.

Lignier, H., C. Sias, D. Ciampini, Y. Singh, A. Zenesini, O. Morsch, and E. Arimondo, 2007, Phys. Rev. Lett. 99, 220403.

Lindner, N. H., G. Refael, and V. Galitski, 2011, Nat. Phys. 7, 490. 
Linz, P., 1985, Analytical and Numerical Methods for Volterra Equations (SIAM, Philadelphia).

Lipavský, P., V. Špička, and B. Velický, 1986, Phys. Rev. B 34, 6933. Liu, M., et al., 2012, Nature (London) 487, 345.

Liu, S. Q., N. J. Wu, and A. Ignatiev, 2000, Appl. Phys. Lett. 76, 2749.

Lubatsch, A., and J. Kroha, 2009, Ann. Phys. (Berlin) 18, 863.

Luttinger, J. M., 1951, Phys. Rev. 84, 814.

Luttinger, J. M., and J. C. Ward, 1960, Phys. Rev. 118, 1417.

Magnus, W., and S. Winkler, 1966, Hill's Equation (John Wiley and Sons, New York).

Mahan, G. D., 1984, Phys. Rep. 110, 321.

Mahan, G. D., 2000, Many-Particle Physics (Plenum, New York), 3rd ed.

Maier, T., M. Jarrell, T. Pruschke, and M. H. Hettler, 2005, Rev. Mod. Phys. 77, 1027.

Manmana, S. R., S. Wessel, R. M. Noack, and A. Muramatsu, 2007, Phys. Rev. Lett. 98, 210405.

Martin, P. C., and J. Schwinger, 1959, Phys. Rev. 115, 1342.

Martinez, D. F., 2003, J. Phys. A 36, 9827.

Martinez, D. F., 2005, J. Phys. A 38, 9979.

Martinez, D. F., and R. A. Molina, 2006, Eur. Phys. J. B 52, 281.

Mathey, L., and A. Polkovnikov, 2010, Phys. Rev. A 81, 033605.

Matsueda, H., and S. Ishihara, 2007, J. Phys. Soc. Jpn. 76, 083703.

Matsueda, H., S. Sota, T. Tohyama, and S. Maekawa, 2012, J. Phys. Soc. Jpn. 81, 013701.

Metropolis, N., A. W. Rosenbluth, M. N. Rosenbluth, A. H. Teller, and E. Teller, 1953, J. Chem. Phys. 21, 1087.

Metzner, W., 1991, Phys. Rev. B 43, 8549.

Metzner, W., and D. Vollhardt, 1989, Phys. Rev. Lett. 62, 324.

Meyer, D., A. C. Hewson, and R. Bulla, 2002, Phys. Rev. Lett. 89, 196401.

Micnas, R., J. Ranninger, and S. Robaszkiewicz, 1990, Rev. Mod. Phys. 62, 113.

Mierzejewski, M., and P. Prelovšek, 2010, Phys. Rev. Lett. 105, 186405.

Mierzejewski, M., V. Vidmar, J. Boncǎ, and P. Prelovsěk, 2011, Phys. Rev. Lett. 106, 196401.

Mikelsons, K., J. K. Freericks, and H. R. Krishnamurthy, 2012, Phys. Rev. Lett. 109, 260402.

Mikelsons, K., A. Macridin, and M. Jarrell, 2009, Phys. Rev. E 79, 057701.

Mitra, A., S. Takei, Y. B. Kim, and A. J. Millis, 2006, Phys. Rev. Lett. 97, 236808.

Mizuno, Y., K. Tsutsui, T. Tohyama, and S. Maekawa, 2000, Phys. Rev. B 62, R4769.

Moeckel, M., and S. Kehrein, 2008, Phys. Rev. Lett. 100, 175702.

Moeckel, M., and S. Kehrein, 2010, New J. Phys. 12, 055016.

Mori, H., 1965, Prog. Theor. Phys. 33, 423.

Mori, T., T. Ozawa, Y. Bando, T. Kawamoto, S. Niizeki, H. Mori, and I. Terasaki, 2009, Phys. Rev. B 79, 115108.

Moritz, B., T. P. Devereaux, and J. K. Freericks, 2010, Phys. Rev. B 81, 165112.

Moritz, B., A. F. Kemper, M. Sentef, T. P. Devereaux, and J. K. Freericks, 2013, Phys. Rev. Lett. 111, 077401.

Mosk, A. P., 2005, Phys. Rev. Lett. 95, 040403.

Mühlbacher, L., and E. Rabani, 2008, Phys. Rev. Lett. 100, 176403.

Müller-Hartmann, E., 1984, Z. Phys. B 57, 281.

Müller-Hartmann, E., 1989a, Z. Phys. B 74, 507.

Müller-Hartmann, E., 1989b, Z. Phys. B 76, 211.

Nakajima, S., 1958, Prog. Theor. Phys. 20, 948.

Nakamura, S., 2010, Prog. Theor. Phys. 124, 1105.

Nakamura, S., 2012, Phys. Rev. Lett. 109, 120602.
Nasu, K., 2004, Ed., Photoinduced Phase Transitions (World Scientific, Singapore).

Negele, J. W., and H. Orland, 1988, Quantum Many-Particle Systems (Addison-Wesley, Redwood City).

Nordlander, P., M. Pustilnik, Y. Meir, N. S. Wingreen, and D. C. Langreth, 1999, Phys. Rev. Lett. 83, 808.

Ogasawara, T., M. Ashida, N. Motoyama, H. Eisaki, S. Uchida, Y. Tokura, H. Ghosh, A. Shukla, S. Mazumdar, and M. Kuwata-Gonokami, 2000, Phys. Rev. Lett. 85, 2204.

Oka, T., 2012, Phys. Rev. B 86, 075148.

Oka, T., and H. Aoki, 2005, Phys. Rev. Lett. 95, 137601.

Oka, T., and H. Aoki, 2008, Phys. Rev. B 78, 241104.

Oka, T., and H. Aoki, 2009a, Lecture Notes in Physics, Vol. 762 (Springer- Verlag, Berlin), p. 251.

Oka, T., and H. Aoki, 2009b, Phys. Rev. B 79, 081406.

Oka, T., and H. Aoki, 2010, Phys. Rev. B 81, 033103.

Oka, T., R. Arita, and H. Aoki, 2003, Phys. Rev. Lett. 91, 066406.

Oka, T., N. Konno, R. Arita, and H. Aoki, 2005, Phys. Rev. Lett. 94, 100602

Okamoto, H., H. Matsuzaki, T. Wakabayashi, Y. Takahashi, and T. Hasegawa, 2007, Phys. Rev. Lett. 98, 037401.

Okamoto, S., 2007, Phys. Rev. B 76, 035105.

Okamoto, S., 2008, Phys. Rev. Lett. 101, 116807.

Oshima, H., K. Miyano, Y. Konishi, M. Kawasaki, and Y. Tokura, 1999, Appl. Phys. Lett. 75, 1473.

Otsuki, J., H. Kusunose, and Y. Kuramoto, 2009, Phys. Rev. Lett. 102, 017202.

Peierls, R., 1933, Z. Phys. 80, 763.

Perfetti, L., P. A. Loukakos, M. Lisowski, U. Bovensiepen, H. Berger, S. Biermann, P. S. Cornaglia, A. Georges, and M. Wolf, 2006, Phys. Rev. Lett. 97, 067402.

Perfetti, L., P. A. Loukakos, M. Lisowski, U. Bovensiepen, M. Wolf, H. Berger, S. Biermann, and A. Georges, 2008, New J. Phys. 10, 053019.

Petersen, J. C., et al., 2011, Phys. Rev. Lett. 107, 177402.

Polkovnikov, A., K. Sengupta, A. Silva, and M. Vengalattore, 2011, Rev. Mod. Phys. 83, 863.

Potthoff, M., M. Aichhorn, and C. Dahnken, 2003, Phys. Rev. Lett. 91, 206402.

Potthoff, M., and W. Nolting, 1999, Phys. Rev. B 59, 2549.

Press, W. H., S. A. Teukolsky, W. T. Vetterling, and B. P. Flannery, 1992, Numerical Recipes in C (Cambridge University Press, Cambridge, England), 2nd ed.

Prokof'ev, N. V., B. V. Svistunov, and I. S. Tupitsyn, 1998, J. Exp. Theor. Phys. 87, 310.

Pruschke, T., D. L. Cox, and M. Jarrell, 1993, Phys. Rev. B 47, 3553.

Pruschke, T., and N. Grewe, 1989, Z. Phys. B 74, 439.

Rammer, J., 1998, Quantum Transport Theory (Perseus Books, New York).

Ramsey, N. F., 1956, Phys. Rev. 103, 20.

Rapp, A., S. Mandt, and A. Rosch, 2010, Phys. Rev. Lett. 105, 220405.

Rigol, M., 2009a, Phys. Rev. Lett. 103, 100403.

Rigol, M., 2009b, Phys. Rev. A 80, 053607.

Rigol, M., V. Dunjko, and M. Olshanii, 2008, Nature (London) 452, 854.

Rigol, M., V. Dunjko, V. Yurovsky, and M. Olshanii, 2007, Phys. Rev. Lett. 98, 050405.

Rini, M., R. Tobey, N. Dean, J. Itatani, Y. Tomioka, Y. Tokura, R. W. Schoenlein, and A. Cavalleri, 2007, Nature (London) 449, 72.

Ritus, V. I, 1966, Zh. Eksp. Teor. Fiz. 51, 1544 [Sov. Phys. JETP 24, 1041 (1967)].

Rubtsov, A. N., M. I. Katsnelson, and A. I. Lichtenstein, 2008, Phys. Rev. B 77, 033101. 
Rubtsov, A. N., V. V. Savkin, and A. I. Lichtenstein, 2005, Phys. Rev. B 72, 035122.

Sadler, L. E., J. M. Higbie, S. R. Leslie, M. Vengalattore, and D. M. Stamper-Kurn, 2006, Nature (London) 443, 312.

Saito, H., Y. Kawaguchi, and M. Ueda, 2007, Phys. Rev. A 75, 013621.

Sambe, H., 1973, Phys. Rev. A 7, 2203.

Sandri, M., M. Schiró, and M. Fabrizio, 2012, Phys. Rev. B 86, 075122.

Sawa, A., T. Fujii, M. Kawasaki, and Y. Tokura, 2004, Appl. Phys. Lett. 85, 4073.

Sawano, F., I. Terasaki, H. Mori, T. Mori, M. Watanabe, N. Ikeda, Y. Nogami, and Y. Noda, 2005, Nature (London) 437, 522.

Scalapino, D. J., S. R. White, and S. C. Zhang, 1992, Phys. Rev. Lett. 68, 2830 .

Schiró, M., and M. Fabrizio, 2009, Phys. Rev. B 79, 153302.

Schiró, M., and M. Fabrizio, 2010, Phys. Rev. Lett. 105, 076401.

Schiró, M., and M. Fabrizio, 2011, Phys. Rev. B 83, 165105.

Schmidt, P., and H. Monien, 2002, arXiv:cond-mat/0202046.

Schmitt, F., et al., 2008, Science 321, 1649.

Schneider, U., L. Hackermüller, S. Will, T. Best, I. Bloch, T. A. Costi, R. W. Helmes, D. Rasch, and A. Rosch, 2008, Science 322, 1520.

Schollwöck, U., 2005, Rev. Mod. Phys. 77, 259.

Schwinger, J., 1951, Phys. Rev. 82, 664.

Schwinger, J., 1961, J. Math. Phys. (N.Y.) 2, 407.

Sciolla, B., and G. Biroli, 2010, Phys. Rev. Lett. 105, 220401.

Sénéchal, D., D. Perez, and M. Pioro-Ladrière, 2000, Phys. Rev. Lett. 84, 522.

Sensarma, R., D. Pekker, E. Altman, E. Demler, N. Strohmaier, D. Greif, R. Jördens, L. Tarruell, H. Moritz, and T. Esslinger, 2010, Phys. Rev. B 82, 224302.

Sentef, M., A. F. Kemper, B. Moritz, J. K. Freericks, Z.-X. Shen, and T. P. Devereaux, 2012, arXiv:1212.4841v3.

Shiba, H., 1972, Prog. Theor. Phys. 48, 2171.

Shim, J. H., K. Haule, and G. Kotliar, 2007a, Nature (London) 446, 513.

Shim, J. H., K. Haule, and G. Kotliar, 2007b, Science 318, 1615.

Shimizu, A., and T. Yuge, 2011, J. Phys. Soc. Jpn. 80, 093706.

Shirley, J. H., 1965, Phys. Rev. 138, B979.

Simon, J., W. S. Bakr, R. Ma, M. E. Tai, P. M. Preiss, and M. Greiner, 2011, Nature (London) 472, 307.

Srednicki, M., 1994, Phys. Rev. E 50, 888.

Stan, A., N. E. Dahlen, and R. van Leeuwen, 2009, J. Chem. Phys. 130, 224101.

Stark, M., and M. Kollar, 2013, arXiv:1308.1610.

Strohmaier, N., D. Greif, R. Jördens, L. Tarruell, H. Moritz, T. Esslinger, R. Sensarma, D. Pekker, E. Altman, and E. Demler, 2010, Phys. Rev. Lett. 104, 080401.

Struck, J., C. Ölschläger, R. Le Targat, P. Soltan-Panahi, A. Eckardt, M. Lewenstein, P. Windpassinger, and K. Sengstock, 2011, Science 333, 996.

Struck, J., C. Ölschläger, M. Weinberg, P. Hauke, J. Simonet, A. Eckardt, M. Lewenstein, K. Sengstock, and P. Windpassinger, 2012, Phys. Rev. Lett. 108, 225304.

Taguchi, Y., T. Matsumoto, and Y. Tokura, 2000, Phys. Rev. B 62, 7015.

Takahashi, A., H. Itoh, and M. Aihara, 2008, Phys. Rev. B 77, 205105

Tarruell, L., D. Greif, T. Uehlinger, G. Jotzu, and T. Esslinger, 2012, Nature (London) 483, 302.

Tokura, Y., 2006, J. Phys. Soc. Jpn. 75, 011001.

Tokura, Y., H. Okamoto, T. Koda, T. Mitani, and G. Saito, 1988, Phys. Rev. B 38, 2215.
Tran, M.-T., 2008, Phys. Rev. B 78, 125103.

Tsuji, N., 2011, Ph.D. thesis, University of Tokyo.

Tsuji, N., P. Barmettler, H. Aoki, and P. Werner, 2013, arXiv: 1307.5946.

Tsuji, N., M. Eckstein, and P. Werner, 2013, Phys. Rev. Lett. 110, 136404.

Tsuji, N., T. Oka, and H. Aoki, 2008, Phys. Rev. B 78, 235124.

Tsuji, N., T. Oka, and H. Aoki, 2009, Phys. Rev. Lett. 103, 047403.

Tsuji, N., T. Oka, H. Aoki, and P. Werner, 2012, Phys. Rev. B 85, 155124.

Tsuji, N., T. Oka, P. Werner, and H. Aoki, 2011, Phys. Rev. Lett. 106, 236401.

Tsuji, N., and P. Werner, 2013, Phys. Rev. B 88, 165115.

Turkowski, V., and J.K. Freericks, 2005, Phys. Rev. B 71, 085104

Turkowski, V., and J. K. Freericks, 2007a, Phys. Rev. B 75, 125110.

Turkowski, V., and J. K. Freericks, 2007b, Strongly Correlated Systems: Coherence and Entanglement (World Scientific, Singapore).

Turkowski, V., and J. K. Freericks, 2008, Phys. Rev. B 77, 205102; 2010, 82, 119904(E).

Turkowski, V. M, and J. K. Freericks, 2006, Phys. Rev. B 73, 075108; 2006, 73, 209902(E).

van Dongen, P. G. J., 1992, Phys. Rev. B 45, 2267.

van Dongen, P. G. J., and D. Vollhardt, 1990, Phys. Rev. Lett. 65, 1663.

Vollhardt, D., 1991, Physica (Amsterdam) 169B, 277.

Vollhardt, D., 1993, in Correlated Electron Systems, edited by V. I. Emery (World Scientific, Singapore), p. 57.

Wagner, M., 1991, Phys. Rev. B 44, 6104.

Wall, S., et al., 2011, Nat. Phys. 7, 114.

Watanabe, S., N. Minami, and R. Shimano, 2011, Opt. Express 19, 1528.

Weiler, C. N., T. W. Neely, D. R. Scherer, A. S. Bradley, M. J. Davis, and B. P. Anderson, 2008, Nature (London) 455, 948.

Werner, P., A. Comanac, L. de' Medici, M. Troyer, and A. J. Millis, 2006, Phys. Rev. Lett. 97, 076405.

Werner, P., and M. Eckstein, 2012, Phys. Rev. B 86, 045119.

Werner, P., and M. Eckstein, 2013, Phys. Rev. B 88, 165108.

Werner, P., and A. J. Millis, 2006, Phys. Rev. B 74, 155107.

Werner, P., and A. J. Millis, 2007, Phys. Rev. Lett. 99, 146404.

Werner, P., T. Oka, M. Eckstein, and A. J. Millis, 2010, Phys. Rev. B 81, 035108 .

Werner, P., T. Oka, and A. J. Millis, 2009, Phys. Rev. B 79, 035320

Werner, P., N. Tsuji, and M. Eckstein, 2012, Phys. Rev. B 86, 205101

White, S., and A. Feiguin, 2004, Phys. Rev. Lett. 93, 076401.

Wigner, E., 1932, Phys. Rev. 40, 749.

Yamada, K., 1975, Prog. Theor. Phys. 53, 970.

Yonemitsu, K., and K. Nasu, 2008, Phys. Rep. 465, 1.

Yosida, K., and K. Yamada, 1970, Prog. Theor. Phys. Suppl. 46, 244.

Yosida, K., and K. Yamada, 1975, Prog. Theor. Phys. 53, 1286.

Yuzbashyan, E. A., and M. Dzero, 2006, Phys. Rev. Lett. 96, 230404.

Zel'dovich, Y. B., 1966, Zh. Eksp. Teor. Fiz. 51, 1492 [Sov. Phys. JETP 24, 1006 (1967)].

Zener, C., 1932, Proc. R. Soc. A 137, 696.

Zener, C., 1934, Proc. R. Soc. A 145, 523.

Zenesini, A., H. Lignier, D. Ciampini, O. Morsch, and E. Arimondo, 2009, Phys. Rev. Lett. 102, 100403. 
Zenesini, A., H. Lignier, G. Tayebirad, J. Radogostowicz, D. Ciampini, R. Mannella, S. Wimberger, O. Morsch, and E. Arimondo, 2009, Phys. Rev. Lett. 103, 090403.

Zhang, X. Y., M. J. Rozenberg, and G. Kotliar, 1993, Phys. Rev. Lett. 70, 1666.

Zurek, W. H., 1985, Nature (London) 317, 505.
Zwanzig, R., 1960, J. Chem. Phys. 33, 1338.

See Supplementary Material at http://link.aps.org/supplemental/ 10.1103/RevModPhys.86.779 for sample programs of the nonequilibrium dynamical mean-field theory reviewed in this article. They are written in both C++ and FORTRAN. For the use of these codes and other details, see Appendix B. 